Aspectos algébricos e computacionais da teoria de bases de Gröbner não comutativas

Alexey Antônio Villas Bôas

\author{
DisSERTAÇÃO APRESENTADA \\ $\mathrm{AO}$ \\ Instituto de Matemática E Estatística \\ DA \\ Universidade de São Paulo \\ PARA \\ obTEnÇÃo Do Título de Mestre \\ EM \\ CiÈnCias
}

Área de Concentração: Ciência da Computação

Orientador: Prof. Dr. Eduardo do Nascimento Marcos

— São Paulo, fevereiro de 2005 - 


\section{Aspectos algébricos e computacionais da teoria de bases de Gröbner não comutativas}

Este exemplar corresponde à redação final da dissertação devidamente corrigida e defendida por Alexey Antônio Villas Bôas e aprovada pela comissão julgadora.

São Paulo, fevereiro de 2005

Banca examinadora:

- Prof. Dr. Eduardo do Nascimento Marcos (IME-USP)

- Prof. Dr. Arnaldo Mandel (IMIE-USP)

- Prof. Dr. Severino Collier Coutinho (UFRJ)

Suplentes:

- Prof. Dr. José Coelho de Pina (IME-LSP)

- Prof. Dr. Renato Portugal (L广CC) 
Aos meus pais, irmãos e arós. 


\section{Agradecimentos}

Gostaria de agradecer primeiramente a meu orientador, Eduardo do Nascimento Marcos, pelo excelente trabalho de orientação. Suas grandes qualidades como matemático e como pessoa tornaram a realização deste trabalho muito prazerosa. Sua paixão pela matemática, sua grande erudição e suas boas idéias sempre fizeram de nossas reuniões uma atividade muito gratificante e eu lhe sou muito grato por sua dedicação, sua paciência com minhas faltas e pelos grandes ensinamentos que me dispensou.

Agradeço em seguida aos professores Arnaldo Mandel e Severino Collier Coutinho por terem aceitado participar da banca examinadora de defesa. É uma imensa honra para mim tê-los nessa banca e espero poder retribuir à altura com um bom trabalho.

Ao professor Arnaldo Mandel, agradeço ainda pelas excelentes sugestões e conselhos. Seu grande conhecimento em Ciência da Computação e em Álgebra trouxe luzes novas para o desenvolvimento deste trabalho e ele certamente seria de menor qualidade não fosse por elas. Suas idéias estão presentes em muitas partes da dissertação e espero saber retribuir honrando seus ensinamentos.

Agradeço ao professor José Coelho de Pina, por ter participado de minha banca de qualificação e pelas excelentes sugestões durante e depois dessa. Deixo também o meu muito obrigado à professora Alegria Gladys C. de Oliveira, por ter me "adotado" como orientando enquanto meu orientador esteve fora do país e por toda atenção que me dispensou antes e depois desse período.

Gostaria aqui de mencionar outros grandes professores que tive a alegria de encontrar e que, embora não tenham tido colaboração direta nesta dissertação, me apresentaram lições valiosíssimas e sempre me inspiraram em meu trabalho. São eles: Carlos Eduardo Ferreira, Francisco César Polcino Milies, Paulo Feofiloff, Nami Kobayashi, Yoshiko Wakabayashi e Israel Jelin.

Pude ainda contar com a ajuda e apoio de muitos amigos durante a realização deste trabalho e a todos eles sou muito grato, embora não haja espaço para mencioná-los todos.

Dentre esses amigos, devo agradecimentos especiais a Rodrigo N. P. Mariano de Souza, a quem aqui chamo de grande amigo e Cientista. Sua contribuição intelectual para este trabalho foi preciosa, bem como foram seus conselhos e seu incentivo. Sem eles, a realização deste trabalho teria sido certamente mais árdua e o resultado final menos interessante.

Gostaria de agradecer também aos grandes amigos Flávio Regis de Arruda, Leandro Farina dos Santos, Thiago Teixeira Santos e a todos os demais integrantes da Ala Oeste, pelo incentivo, amizade e apoio durante toda a graduação e o mestrado.

Gostaria de agradecer a Peterson Pereira de Oliveira pelas discussões sobre a Teoria de Bases de Gröbner e pela boa troca de experiências que vieram delas.

Agradeço ainda aos bons amigos de São Bernardo, Ana Lúcia Marreiros, Francisco Elói S. de Araújo, Jacqueline A. de Oliveira e muitos outros que colaboraram tanto por meio da leitura de trechos do texto, quanto com sugestões, conselhos e incentivo.

Agradeço ainda à Praça do Oceanográfico pelas boas idéias; à pequena cidade de Itanhaém pelas horas prazerosas de trabalho e ao Mar pela paz nos momentos difíceis.

Gostaria de deixar o meu muito obrigado a Melina Silveira Rossi, a minha querida Nina, por iluminar o meu caminho com sua luz e seu carinho.

Agradeço ainda aos meus irmãos Alessandra e Cyrus e a meus avós Leris e Mário e Yolanda e 
Antônio. Sua presença em vida ou em lembrança sempre foram de imenso valor para mim e, sem dúvida, constituíram um grande incentivo para a realização deste trabalho.

Por fim, mas certamente não menos importante, gostaria de agradecer a meus pais Edgar e Sandra, cujos ensinamentos são responsáveis por grande parte do que sou hoje. O Amor e a Sabedoria que me dispensaram estão presentes por toda minha vida e seus nomes merecem menção em todas as minhas obras.

Alexey Antônio Villas Bôas 


\section{Resumo}

A Teoria de Bases de Gröbner foi introduzida no contexto do anel de polinômios comutativo por Bruno Buchberger em sua tese de doutorado em 1965. Essa teoria encontra suas primeiras aplicações na área de Geometria Algébrica e possui um papel central em Álgebra Computacional. Grande parte de sua importância se deve ao fato de ela fornecer uma solução algorítmica para o problema da pertinência em ideais do anel de polinômios comutativo. Os dois principais algoritmos presentes na teoria são o Algoritmo da Divisão e o Algoritmo de Buchberger.

Alguns anos mais tarde, essa teoria foi generalizada por Mora para o caso não comutativo (mais precisamente para as álgebras livres) e posteriormente para contextos mais gerais, tais quais as álgebras de caminhos. Em particular, Mora apresentou uma generalização do Algoritmo de Buchberger, que é conhecida na literatura como Procedimento de Mora.

Este trabalho tem o intuito de apresentar uma introdução à Teoria de Bases de Gröbner não comutativas. Apresentamos de forma bastante breve uma motivação para o estudo dessa teoria e um panorama dela para o caso comutativo. Em seguida, fornecemos as bases da teoria, suas principais definições e resultados mais fundamentais em um contexto mais amplo que o das álgebras livres.

Um importante resultado devido a Farkas, Feustel e Green mostra que toda álgebra para a qual se pode desenvolver uma teoria de Bases de Gröbner não comutativas é isomorfa a um quociente de álgebra de caminhos. Incluímos esse resultado, acompanhado de uma demonstração detalhada.

Outro resultado central afirma que um subconjunto $G$ de uma álgebra de caminhos é uma Base de Gröbner se e somente se todas as relações de sobreposição e de divisão envolvendo seus elementos se reduzem para zero sobre $G$ (por meio do Algoritmo da Divisão). Em outras palavras, esse resultado fornece um teste algorítmico para decidir quando um conjunto gerador (finito) de um ideal é uma Base de Gröbner. Esse teorema foi demonstrado primeiramente por Buchberger para o caso comutativo, em seguida por Mora para as álgebras livres e posteriormente por Green para as álgebras de caminhos. Apresentamos aqui uma demonstração para esse resultado (em sua versão para as álgebras de caminhos) alternativa à dada por Green. A prova aqui vista é uma generalização do argumento de Mora para as álgebras de caminhos.

O trabalho traz também discussões detalhadas da versão não comutativa do Algoritmo da Divisão e do Procedimento de Mora. Em particular, apresentamos duas versões bastante presentes na literatura para o último, todas acompanhadas de suas provas de correção.

Discutimos ainda algumas das principais questões de computabilidade relacionadas com a Teoria de Bases de Gröbner não comutativas e outros resultados simples, mas não presentes na literatura.

Finalmente, apresentamos uma introdução ao conceito de Base de Gröbner Regular criado por Mansson e Nordbeck. Discutiremos a importância dessas bases e apresentamos para elas uma definição alternativa àquela dada por seus criadores. Nossa definição faz uso do conceito bem estudado de relação racional e, em certo sentido, parece ser mais natural que a definição original. 


\begin{abstract}
Gröbner Bases were introduced by Bruno Buchberger in his Ph.D thesis in 1965. Their study was first motivated by problems in the field of Algebraic Geometry and they have played a major role in Computational Algebra ever since. Most of its importance stems from the fact that Gröbner Bases provide an algorithmic solution to the ideal membership problem in the commutative polynomial ring. This solution is given by two algorithms, namely the Division Algorithm and Buchberger's Algorithm.

The theory of Gröbner Bases was first generalized to the context of the free algebra by Mora and later to more general contexts, such as path algebras. Mora presented a generalization of Buchberger's Algorithm to the non-commutative case, which is known as Mora's Procedure.

In this work, we study the theory of non-commutative Gröbner Bases. To begin with, we show some motivation and an overview of the theory in the commutative case. We then present a detailed introduction to the basic definitions and results of the non-commutative version of the theory.

An important result due to Farkas, Feustel and Green states that every algebra for which it is possible to develop the theory of non-commutative Gröbner Bases is isomorphic to a quotient of a path algebra. We include this result in our work and show a detailed proof for it.

Another important result shows that a generating subset $G$ for an ideal in a path algebra is a Gröbner Basis if and only if every overlap and division relation involving elements in $G$ reduce to zero over $G$ (by means of the Division Algorithm). This fundamental result was first stated by Buchberger for the commutative case. It was then generalized to the free algebra case by Mora and later to path algebras by Green. We include in this work a proof for this result (for the path algebra context) that differs from that given by Green. Our approach follows the argument of Mora and provides a generalization of it to path algebras.

We also discuss thoroughly the Division Algorithm and Mora's Procedure. Two well-known versions of the latter are presented, and we prove the correctness of all the algorithms discussed. We also discuss some topics related to computability of Gröbner Bases and other minor results not seen in the literature.

Finally, we give an introduction to the concept of Regular Gröbner Basis, presented by Mansson and Nordbeck. We discuss the basic properties and the importance of these bases and present an alternative definition for them. Our definition of Regular Gröbner Basis makes use of the wellstudied concept of rational relation and seems to be more natural than the original one.
\end{abstract}




\section{ÍNDICE}

Introdução $\quad$ v v v v v v

1 Preliminares 1

1.1 Conjuntos, Ordens e Relações . . . . . . . . . . . . . . . . . . . . 1

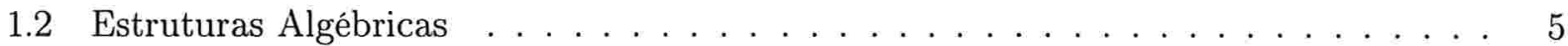

1.3 Grafos . . . . . . . . . . . . . . . . . . . . . . . . 12

1.4 Álgebras de Caminho . . . . . . . . . . . . . . . . . . . . . . . 14

1.5 Linguagens Formais e Autômatos . . . . . . . . . . . . . . . . . . . 16

1.6 Algoritmos e Computabilidade . . . . . . . . . . . . . . . . . . . . . 21

2 Motivação e Bases de Gröbner Comutativas $\quad 25$

2.1 Histórico e Motivação . . . . . . . . . . . . . . . . . . . . 25

2.2 O Problema da Pertinência em Subespaços Vetoriais . . . . . . . . . . . . . . 26

2.3 O Problema da Pertinência em $K[x] \ldots \ldots \ldots \ldots \ldots \ldots \ldots \ldots$

2.4 Bases de Gröbner Comutativas . . . . . . . . . . . . . . . . . . . . . 33

3 Introdução a Bases de Gröbner não Comutativas $\quad 45$

3.1 Conceitos Iniciais . . . . . . . . . . . . . . . . . . . . . . 47

3.2 Bases de Gröbner . . . . . . . . . . . . . . . . . . . . . . 59

3.3 Álgebras de Dimensão Finita . . . . . . . . . . . . . . . . . . . . . . 67

3.4 Propriedades Básicas e Álgebras de Caminhos . . . . . . . . . . . . . . . . 73

3.4 .1 Resultados Iniciais . . . . . . . . . . . . . . . . . . . . . . 74

3.4 .2 Congruências . . . . . . . . . . . . . . . . . . . . 80

3.4 .3 Demonstração do Teorema $3.4 .11 \ldots \ldots$. . . . . . . . . . . . 90

3.4 .4 Algumas Considerações . . . . . . . . . . . . . . . . . . . . . . . . 91

4 Algoritmos e Outros Aspectos Computacionais $\quad 99$

4.1 O Algoritmo da Divisão . . . . . . . . . . . . . . . . . . . . . . . . . . . . 99

4.1 .1 O Algoritmo da Divisão no Caso Geral . . . . . . . . . . . . . . . . . . . 100

4.1 .2 O Algoritmo da Divisão em Álgebras de Caminhos . . . . . . . . . . . . . . . 113

4.2 Fundamentos Algébricos do Procedimento de Mora . . . . . . . . . . . . . . . . . . 114

4.2 .1 Uniformidade . . . . . . . . . . . . . . . . . . . . . . 115 
4.2 .2 Resultados Principais . . . . . . . . . . . . . . . . . . . 118

4.2 .3 S-elementos e Sobreposições . . . . . . . . . . . . . . . . . . . . 129

4.2 .4 Conclusões . . . . . . . . . . . . . . . . . . . . . . . . . 134

4.2 .5 Um Contra-exemplo . . . . . . . . . . . . . . . . . . . . . 136

4.2 .6 Variações sobre o Lema do Diamante . . . . . . . . . . . . . . . . . 137

4.2 .7 Bases que não Admitem Ordens Admissíveis . . . . . . . . . . . . . . . . 144

4.3 O Procedimento de Mora . . . . . . . . . . . . . . . . . . 146

4.3 .1 Uma Primeira Versão . . . . . . . . . . . . . . . . . . . . . . 147

4.3 .2 Tl-Redução . . . . . . . . . . . . . . . . . . . . . . . . . . 155

4.3 .3 Uma Segunda Versão . . . . . . . . . . . . . . . . . . . . . . 163

4.3.4 Bases de Gröbner Reduzidas . . . . . . . . . . . . . . . 170

4.4 Computabilidade de Bases de Gröbner . . . . . . . . . . . . . 171

5 Bases de Gröbner Regulares $\quad 177$

5.1 Conceitos Iniciais . . . . . . . . . . . . . . . . . . . . . 177

5.2 Álgebras de Autômato . . . . . . . . . . . . . . . . . . . . . . . . . . . . . . . . . . . . . . . . . . . .

5.3 Relações Racionais . . . . . . . . . . . . . . . . . . . . . . . 183

5.4 Bases de Gröbner Regulares . . . . . . . . . . . . . . . . . . . . . . . . . . 189

5.5 Boas Representações e Reduções . . . . . . . . . . . . . . . . . . . . 198

5.6 Considerações Finais . . . . . . . . . . . . . . . . . . . . . . . . 199

$\begin{array}{ll}\text { Referências Bibliográficas } & 201\end{array}$

$\begin{array}{ll}\text { Indice Remissivo } & 205\end{array}$ 


\section{LisTA DE Algoritmos}

2.2 .1 - Algoritmo de Gauss-Jordan (GaussJordan) . . . . . . . . . . . . . . 27

2.2 .2 - Redução Incompleta de Gauss (RedincGauss) . . . . . . . . . . . . . . . 30

2.3.1 - Algoritmo da Divisão para Polinômios em $K[x]$ (DIvisãoPoli) . . . . . . . 32

2.3 .2 - Algoritmo de Euclides (EuCLIDES) $\ldots \ldots \ldots \ldots$

2.4 .1 - Algoritmo da Divisão em $K\left[x_{1}, \ldots, x_{n}\right]$ (DivisãoCoмut) . . . . . . . . 36

2.4 .2 - Algoritmo de Buchberger (BUCHBERGER) . . . . . . . . . . . . . . 41

4.1 .1 - Algoritmo da Divisão (DIVISÃo) . . . . . . . . . . . . . . 100

4.3 .1 - Procedimento de Mora (PMORA) . . . . . . . . . . . . . . . . 147

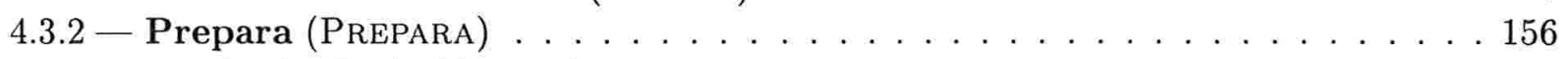

4.3 .3 - TL-Redução (TLREduz) … . . . . . . . . . . . . . . 157

4.3.4 - Segunda versão do Procedimento de Mora (PMora2) . . . . . . . . . . 164

4.3 .5 - Base de Gröbner Reduzida (BGREDUzIDA) . . . . . . . . . . . . 171 



\section{INTRODUÇÃO}

A Teoria de Bases de Gröbner surgiu no contexto do anel de polinômios comutativo, tendo como principal motivação problemas em Geometria Algébrica. As idéias envolvidas nessa teoria foram exploradas por Hironaka [Hir64] e, independentemente, um pouco mais tarde por Buchberger [Buc65]. Buchberger cunhou o termo "Base de Gröbner" e desenvolveu os principais pontos da teoria.

Desde então, o número de estudos feitos nessa área, incluindo generalizações, refinamentos e desenvolvimento de aplicações tem se multiplicado. Essa teoria constitui hoje uma ferramenta fundamental na área de Geometria Algébrica e em outras áreas da Álgebra e um marco no desenvolvimento da Álgebra Computacional.

Grande parte de sua importância reside no fato de que essa teoria fornece uma solução algorítmica para o problema da pertinência em ideais do anel de polinômios comutativo em muitas variáveis, $K\left[x_{1}, \ldots, x_{n}\right]$. Na realidade, a partir de um conjunto (finito) de geradores para um ideal $I$ desse anel, elas fornecem um meio algorítmico de encontrar representantes de classe canônicos para a classes de equivalência do quociente $K\left[x_{1}, \ldots, x_{n}\right] / I$.

Assim, dado um polinômio $p$ em $K\left[x_{1}, \ldots, x_{n}\right]$, é possível associar a $p$ um único polinômio $q$ em $p+I$, que é a forma normal de $p$ (e encontrá-lo algoritmicamente). A forma normal de $p$ é zero se e somente se $p$ pertence a $I$ e dois polinômios possuem a mesma forma normal se e somente se suas projeções no quociente $K\left[x_{1}, \ldots, x_{n}\right] / I$ são iguais. Com isso, a Teoria de Bases de Gröbner fornece um sistema de representantes para as classes de $K\left[x_{1}, \ldots, x_{n}\right] / I$ especialmente bom para efetuar computações no quociente.

Para encontrar formas normais, a Teoria de Bases de Gröbner se utiliza de dois principais algoritmos. O primeiro, o Algoritmo de Buchberger, recebe como entrada um conjunto finito de geradores para um ideal $I$ de $K\left[x_{1}, \ldots, x_{n}\right]$ e devolve um conjunto gerador $G$ para esse mesmo ideal com algumas propriedades adicionais. Esse conjunto $G$ é chamado de uma Base de Gröbner para $I$.

Uma vez que se tenha em mãos uma Base de Gröbner para $I$, um segundo algoritmo, chamado de Algoritmo da Divisão, utiliza essa Base de Gröbner para encontrar as formas normais de elementos de $K\left[x_{1}, \ldots, x_{n}\right]$ com respeito a $I$. O nome Algoritmo da Divisão é apropriado, uma vez que esse algoritmo é uma generalização razoavelmente imediata do bem conhecido algoritmo da divisão de polinômios utilizado para dividir um polinômio por outro.

A teoria introduzida por Buchberger sofreu uma importante generalização para o caso não comutativo. As bases de tal generalização foram inicialmente estudadas por Bergman [Ber78] e por Bokut [Bok76], mas o primeiro a abordá-la de um ponto de vista mais construtivo foi Mora [Mor86]. Mora generalizou os algoritmos de Buchberger e da Divisão para as álgebras livres, introduzindo um procedimento conhecido na literatura como Procedimento de Mora. ${ }^{1}$

\footnotetext{
${ }^{1}$ O Procedimento de Mora é a generalização do Algoritmo de Buchberger para o caso não comutativo. A genera-
} 
Desde então, generalizações e adaptações da teoria para outros contextos foram feitas. Dentre essas, destacamos o caso das álgebras de caminhos, estudado por Farkas, Feustel e Green [FFG93, Gre97]. Na realidade, as definições básicas da teoria podem ser estabelecidas para álgebras arbitrárias. Entretanto, até onde é de conhecimento dos autores, o contexto mais geral para o qual foi generalizado o Procedimento de Mora são as álgebras de caminhos.

De fato, apresentamos um exemplo não visto na literatura (Seção 4.2.5) que mostra que esse procedimento não pode ser generalizado para álgebras arbitrárias sem sofrer significativas modificações.

Com isso, a Teoria de Bases de Gröbner não comutativas têm se mostrado uma área bastante ativa de pesquisa e com aplicações muito promissoras. Algumas delas podem ser vistas no estudo de séries de Hilbert e de Poincaré [Ufn80, Ufn89], bem como no estudo de módulos e de resoluções projetivas [Gre97], entre outros.

Por outro lado, é importante notar que a generalização da teoria para o caso não comutativo encontra muitos percalços. Ao se lidar com o anel de polinômios comutativo, o Teorema da Base de Hilbert fornece a garantia de que todo ideal possui uma Base de Gröbner finita (Teorema 2.4.6). Contudo, esse teorema não é valido no caso não comutativo. Como conseqüência, é possível encontrar ideais da álgebra livre que não possuem nenhuma Base de Gröbner finita.

À primeira vista, essa afirmação não deve vir como uma grande surpresa, uma vez que uma Base de Gröbner para um ideal $I$ é um conjunto gerador para $I$ e é sabido que existem ideais da álgebra livre que não são finitamente gerados. Porém, existem exemplos de ideais finitamente gerados que não admitem nenhuma Base de Gröbner finita (Seção 3.2).

É claro que conjuntos infinitos são sempre uma má notícia para aqueles interessados em lidar com aspectos computacionais. Dessa forma, o Procedimento de Mora pode falhar na busca por uma Base de Gröbner. Na realidade, ele sempre produz uma Base de Gröbner como saída. Contudo, uma vez que essas podem ser conjuntos infinitos, existem situações em que o Procedimento de Mora não termina sua computação.

Mais forte que isso é um fato demonstrado por Mora [Mor94, Mor91] que elimina qualquer esperança de encontrar um algoritmo (que sempre termine sua computação) que compute Bases de Gröbner. O resultado de Mora utiliza uma redução envolvendo o problema da palavra em semigrupos e mostra que o problema de construir Bases de Gröbner no caso não comutativo não é computável (Seção 4.4).

Este trabalho de mestrado dedica-se ao estudo da Teoria de Bases de Gröbner não comutativas. Desse modo, o leitor encontra aqui todas as definições básicas da teoria e um exame detalhado de seus resultados fundamentais. Apresentaremos as bases da teoria no contexto bastante amplo de uma álgebra qualquer, associativa, não comutativa e com unidade. O Algoritmo da Divisão e o Procedimento de Mora serão discutidos no contexto das álgebras de caminhos.

Diz-se que uma álgebra $R$ possui teoria de Bases de Gröbner se ela contém os elementos necessários para o desenvolvimento da teoria (Seção 3.1). Casos bem estudados de álgebras que admitem teoria de Bases de Gröbner são as já mencionadas álgebras livres e álgebras de caminhos. Entretanto, tais elementos não são presentes em todas as álgebras (e, em alguns casos, mesmo que eles sejam presentes, não se sabe como encontrá-los).

Interessante ressaltar que se assumirmos como hipótese que o quociente da álgebra $R$ por um ideal $I$ possui dimensão finita, alguns resultados extras são possíveis (Seção 3.3). Em particular, $I$

lização do Algoritmo da Divisão é bastante imediata, de forma que é comum utilizar o mesmo nome para indicar as duas versōes do algoritmo. 
sempre possui uma Base de Gröbner finita e, mais que isso, sempre possui uma Base de Gröbner universal finita. Ainda, mostramos o resultado não presente na literatura de que, dados um corpo $K$ e uma dimensão $n$, existem apenas um número finito de álgebras não isomorfas sobre $K$ e de dimensão $n$ que admitem teoria de Bases de Gröbner.

Um resultado importante devido a Farkas, Feustel e Green [FFG93, Gre97] é o fato de que toda álgebra que admite uma teoria de Bases de Gröbner é isomorfa a um quociente de álgebra de caminhos por um ideal com certas propriedades especiais (Teorema 3.4.11). Apresentamos a demonstração para esse resultado e comentamos sobre algumas conseqüências que ele traz (Seção 3.4.4).

Ainda, exploraremos as propriedades do Algoritmo da Divisão e do Procedimento de Mora, fornecendo demonstrações para suas correções e apresentando suas principais propriedades (Capítulo 4).

Mora [Mor94] generalizou (para as álgebras livres) um importante resultado de Buchberger que fornece um critério para decidir se um conjunto gerador de um ideal é uma Base de Gröbner (Teorema 4.2.31). Esse resultado é uma peça chave do Procedimento de Mora e foi generalizado por Green para as álgebras de caminhos [Gre97]. Apresentamos uma demonstração alternativa à dada por Green, que segue mais de perto o argumento de Mora, generalizando-o para as álgebras de caminhos. Acreditamos que a demonstração dada ạqui seja mais explícita que a de Green.

Exploraremos também a estreita ligação entre o Lema do Diamante de Bergman [Ber78] e a Teoria de Bases de Gröbner não comutativas, mostrando como esse importante resultado pode ser utilizado para demonstrar o Teorema 4.2.31, mencionado anteriormente (Seção 4.2.6).

Diante das dificuldades que surgem na generalização da Teoria de Bases de Gröbner para o caso não comutativo mais geral, é comum encontrar adaptações para classes de álgebras em que essas dificuldades se tornam mais tratáveis [Mor94, KRW90].

Uma dessas tentativas consiste em encontrar classes de álgebras em que as Bases de Gröbner (infinitas ou não) possam ser representadas por alguma estrutura finita, de modo que essa estrutura possa então ser utilizada em conjunto com o Algoritmo da Divisão para encontrar formas normais.

Nesse sentido, Mansson e Nordbeck introduziram o conceito de Base de Gröbner regular [MN02]. Em linhas gerais, uma Base de Gröbner regular é uma Base de Gröbner que pode ser representada por um autômato finito. Dessa forma, é possível utilizá-la em conjunto com o Algoritmo da Divisão, mesmo que se trate de um conjunto infinito.

Abordaremos neste trabalho os principais conceitos relacionados a essas bases e apresentaremos alguns problemas (até onde sabemos) não resolvidos relacionados a elas (Capítulo 5). Vale mencionar que a definição apresentada aqui difere da original dada por Mansson e Nordbeck. Nossa definição faz uso do bem estudado conceito de relação racional (Seção 5.3) e acreditamos que ela possua algumas vantagens sobre a definição original. Ademais, uma certa "equivalência" entre essas duas formas de ver as Bases de Gröbner regulares é demonstrada (Proposição 5.4.1).

Assim, o restante do texto encontra-se estruturado da seguinte maneira:

Capítulo 1 - Preliminares Apresentamos conceitos preliminares que serão utilizados livremente ao longo de todo o texto e fixamos a notação.

Capítulo 2 - Motivação e Bases de Gröbner Comutativas Este capítulo contém uma introdução à Teoria de Bases de Gröbner comutativas e alguma motivação. Em particular, apresentamos alguns elementos da teoria, considerando o problema da pertinència em contex- 
tos bastante familiares. Nesta parte, temos o propósito de desenvolver uma intuição acerca de alguns dos principais conceitos da teoria, antes de apresentar sua formalização.

Ressaltamos que este capítulo pode ser especialmente interessante para o leitor não familiarizado com a Teoria de Bases de Gröbner, uma vez que ele procura apresentar de maneira rápida todos os elementos de sua versão comutativa. Com isso, uma vez que os detalhes são deixados de lado, o capítulo fornece uma boa descrição da linha de desenvolvimento seguida nos demais capítulos.

Capítulo 3 - Introdução a Bases de Gröbner não Comutativas Apresenta as principais definições e resultados da Teoria de Bases de Gröbner não comutativas.

Capítulo 4 - Algoritmos e Outros Aspectos Computacionais Neste capítulo são discutidos o Algoritmo da Divisão e o Procedimento de Mora. Apresentamos duas versões para esse último muito presentes na literatura. A Seção 4.4 discute ainda algumas questões de computabilidade relacionadas com a teoria.

Capítulo 5 - Bases de Gröbner Regulares Este capítulo fornece uma introdução ao conceito de Base de Gröbner regular. Nele também é abordado o conceito de relação racional (utilizado para definir a noção de Base de Gröbner regular) e algumas de suas propriedades básicas.

É importante ressaltar que, mesmo correndo o risco de sermos prolixos, preferimos abordar os principais assuntos da teoria com detalhes. Este texto foi escrito com a preocupação de fornecer argumentos completos (na maior parte), tendo em vista um leitor interessado em aprender a teoria e sem grande maturidade nas disciplinas de Álgebra e Computação (tomamos como referência um leitor no final de sua graduação ou início de mestrado).

Dessa maneira, um leitor mais maduro pode encontrar no texto certa repetição, proveniente da tentativa de sermos didáticos. Em particular, recomenda-se a um tal leitor que deixe de lado partes mais iniciais do texto ou que recorra a elas sob demanda (em particular, com relação aos Capítulos 1 e 2 ).

Ainda, não recomendamos a nenhum leitor que siga o Capítulo 1 linearmente, mas sim que inicialmente percorra os conceitos apresentados brevemente e volte a consultá-lo conforme necessário.

Encerramos esta introdução citando brevemente os tópicos que acreditamos serem algumas pequenas contribuições deste trabalho.

- Apresentamos alguns pequenos resultados e demonstrações alternativas que (até onde é de nosso conhecimento) não são presentes na literatura. São eles a Proposição 3.3.8 e os resultados apresentados nas Seções 3.4.4 e 4.2.7.

- A demonstração apresentada para o Teorema 4.2.31 é uma generalização não presente na literatura da demonstração de Mora para este mesmo resultado restrito às álgebras livres [Mor94].

- Não conhecemos nenhum exemplo na literatura que mostre que o Teorema 4.2.31 não pode ser generalizado para álgebras quaisquer sem modificações (Seção 4.2.5). ${ }^{2}$

\footnotetext{
${ }^{2}$ Este exemplo foi obtido em colaboração com E. L. Green.
} 
- A demonstração apresentada para a Proposição 5.2.1 difere daquela fornecida por Ufnarovski [Ufn89] e aparenta ser mais simples que a original.

- Apresentamos uma nova definição para o conceito de Base de Gröbner regular, não presente na literatura (Seção 5.4). Acreditamos que a definição apresentada aqui é mais natural que a original [MN02]. 


\section{PRELIMINARES}

Este capítulo traz uma série de conceitos, definições e notações que serão utilizados livremente em todo o restante do texto. Grande parte dos tópicos aqui apresentados deve ser bastante familiar a muitos leitores. Contudo, mesmo correndo o risco de sermos repetitivos, revisitaremos tais conceitos a fim de atender àqueles não familiarizados com eles e de tornar a notação e terminologia do trabalho mais uniforme.

Por outro lado, vale alertar que não temos nenhuma pretensão de fornecer um tratamento completo dos assuntos aqui abordados. Nossas exposições limitam-se ao necessário para fornecer bons subsídios para a compreensão do restante do texto.

A Seção 1.1 apresenta terminologias e resultados envolvendo conjuntos, ordens e relações que serão utilizados extensivamente ao longo de todo o texto. O leitor não fluente nesses tópicos é fortemente incentivado a examinar cuidadosamente essa seção. Em seguida, a Seção 1.2 introduz as principais estruturas algébricas que serão abordadas no restante do trabalho.

A Seção 1.3 apresenta definições básicas envolvendo grafos. A notação vista nessa seção será importante para a apresentação das álgebras de caminhos. Essas são provavelmente a estrutura algébrica mais presente em todo o texto e são discutidas na Seção 1.4.

A Seção 1.5 apresenta fatos bastante conhecidos sobre a Teoria de Linguagens Formais e Autômatos. Prevenimos o leitor que os conceitos apresentados nessa seção serão necessários exclusivamente no Capítulo 5. Dessa forma, sua leitura pode ser deixada de lado por quem não se interesse por esse capítulo sem qualquer prejuízo para o restante do texto.

Finalmente, a Seção 1.6 traz algumas questões relacionadas a computabilidade e à notação que utilizamos para descrever algoritmos.

\subsection{Conjuntos, Ordens e Relações}

Apresentamos aqui alguns conceitos sobre conjuntos, ordens e relações que serão muito utilizados em todo o texto. Maiores detalhes sobre os assuntos aqui apresentados podem ser vistos em [Hal74] e no Capítulo 4 de [BW93].

Denotaremos por $\mathbb{Z}$ o conjunto de todos os inteiros, por $\mathbb{N}$ o conjunto dos inteiros não negativos, isto é, $\mathbb{N}=\{0,1,2, \ldots\}$ e por $\mathbb{N}^{*}$ o conjunto dos inteiros positivos ou naturais não nulos: $\mathbb{N}^{*}=$ $\{1,2, \ldots\}$. Como é usual, $\mathbb{Q}$ denotará o conjunto dos números racionais.

Dado um conjunto $X$, denotaremos por $\mathcal{P}(X)$ o conjunto das partes de $X$, isto é, o conjunto formado por todos os subconjuntos de $X$. Denotaremos ainda por $\operatorname{Fin}(X)$ o conjunto das partes finitas de $X$, ou seja Fin $(X)$ é o conjunto formado por todos os subconjuntos finitos de $X$. 
Denotarmos por $\operatorname{card}(X)$ a cardinalidade de $X$. No caso de $X$ ser um conjunto infinito, diremos que $\operatorname{card}(X)=\infty$.

Uma partição de $X$ é um subconjunto $P=\left\{X_{1}, X_{2}, \ldots\right\}$ de $\mathcal{P}(X)$, de modo que $X_{i} \cap X_{j}=\emptyset$, se $i \neq j$ e $\bigcup_{X_{i} \in P} X_{i}=X$.

Uma operação binária - em $A$ é simplesmente uma função de $A \times A$ em $A$. Como é usual, denotaremos a imagem de $(x, y)$ pela operação $\cdot$ por $x \cdot y$, ou por $x y$, quando não houver possibilidade de confusão.

Dados conjuntos $A$ e $B$, uma relação de $A$ para $B$ é simplesmente um subconjunto $R$ do produto cartesiano $A \times B$. No caso em que $A=B$, diremos que $R$ é uma relação em $A$ (ou em $B$ ). As notações $(x, y) \in R$ e $x R y$ são absolutamente equivalentes.

Algumas vezes será conveniente ver uma relação $R$ de $A$ em $B$ como uma função de $A$ em $\mathcal{P}(B)$. Nesse caso, denotaremos por $R(x):=\{y \in B \mid(x, y) \in R\}$. É claro que a cada subconjunto de $A \times B$ está associada uma única função de $A$ em $\mathcal{P}(B)$. Desse modo, não faremos distinção entre essas duas formas de tratar relações, e utilizaremos sempre a maneira mais conveniente.

O domínio da relação $R$ é o conjunto dado por $\operatorname{Dom}(R):=\{a \in A \mid R(a) \neq \emptyset\}$ e sua imagem o conjunto $\operatorname{Im}(R):=\{b \in B \mid$ existe $a \in A$ tal que $b \in R(a)\}$.

Uma relação $R$ de $A$ em $B$ pode ser estendida para uma aplicação de $\mathcal{P}(A)$ em $\mathcal{P}(B)$, da seguinte forma ( $C$ é um subconjunto de $A$ ): $R(C):=\cup_{a \in C} R(a)$.

É claro que uma função de $A$ em $B$ pode ser vista como uma relação $R$ de $A$ em $B$ satisfazendo $\operatorname{card}(\{(c, b) \in R \mid c=a$ e $b \in B\})=1$, para cada $a$ em $A$.

Dadas duas relações: $S$ de $A$ em $B$ e $T$ de $B$ em $C$, a composição de $S$ e $T$ é a relação $T$ o $S$ (ou simplesmente $T S$, quando o contexto tornar claro que nos referimos a composição de relações) de $A$ em $C$ definida por

$$
T \circ S:=\{(a, c) \in A \times C \mid \text { existe } b \in B \text { tal que }(a, b) \in S \text { e }(b, c) \in T\}
$$

É um fato bem conhecido que a composição de relações é associativa.

Dados um conjunto $A$ e uma relação $R$ em $A$, diremos que $R$ é reflexiva se $x R x$, para todo $x$ em $A$. A relação $R$ é dita simétrica se $x R y$ implica $y R x$, para todos $x$ e $y$ em $A$. Ainda, $R$ é dita transitiva se $x R y$ e $y R w$ implicam $x R w$, para todos $x, y$ e $w$ em $A$. Uma relação que seja reflexiva, simétrica e transitiva é dita uma relação de equivalência.

Dada uma relação qualquer $R$ em $A$, é possível encontrar a menor relação de equivalência que contém $R$. Para tanto, introduzimos os seguintes conceitos.

Dado um conjunto $A$, a diagonal de $A$ (também chamada de relação identidade) é a relação definida por $D_{A}:=\{(a, a) \mid a \in A\}$. Dada uma relação $R$ em $A$, note que $D_{A} \circ R=R \circ D_{A}=R$. Então, definimos as potências de $R$ recursivamente, da seguinte forma:

$$
\begin{aligned}
& R^{0}:=D_{A} \\
& R^{n}:=R^{n-1} \circ R, \text { para todo } n \geq 1
\end{aligned}
$$

Assim, podemos definir:

- A inversa de $R$, dada por $R^{-1}:=\{(a, b) \mid(b, a) \in R\}$.

- O fecho simétrico de $R$, dado por $R \cup R^{-1}$.

- O fecho reflexivo de $R$, dado por $R \cup D_{A}$. 
- O fecho transitivo de $R$, dado por $R^{(+)}:=\cup_{i=1}^{\infty} R^{i}$.

- O fecho reflexivo-transitivo de $R$, dado por $R^{(\infty)}:=\cup_{i=0}^{\infty} R^{i}$.

Com isso, dada uma relação $R$ sobre $A, E_{R}:=\left(R \cup R^{-1}\right)^{(\infty)}$ é a menor relação de equivalência de $A$ que contêm $R$. Mais precisamente, $E_{R}$ é a intersecção de todas as relações de equivalência que contêm $R$. Diremos então que $E_{R}$ é a relação de equivalência gerada por $R$.

Tomemos uma relação de equivalência $R$ em $A$. Então, para cada elemento $a$ de $A$, podemos definir sua classe de equivalência: $[a]_{R}:=\{x \in A \mid x R a\}$. Dada uma classe de equivalência $[a]_{R}$, um elemento qualquer desse conjunto é um representante da classe $[a]_{R}$. O conjunto de todas as classes de equivalência de elementos de $A$ forma uma partição de $A$, denominada quociente de $A$ por $R$ e denotada por $A / R$. Assim, vemos que uma relação de equivalência $R$ em $A$ induz uma partição em $A$. Inversamente, é bem conhecido o fato de que qualquer partição de $A$ induz uma relação de equivalência em $A$.

Se temos uma operação binária - fixada em $A$, a relação $R$ é compatível com o produto de $A$ quando $x R y$ implica $(x \cdot w) R(y \cdot w)$ e $(w \cdot x) R(w \cdot y)$, para todo $w$ em $A$. Uma relação de equivalência em $A$ compatível com o produto de $A$ é dita uma congruência em $A$.

Dada uma relação de equivalência $R$ em $A$, podemos definir seu fecho multiplicativo como

$$
M_{R}:=\{(c a, c b) \mid(a, b) \in R \text { e } c \in A\} \cup\{(a c, b c) \mid(a, b) \in R \text { e } c \in A\}
$$

Assim, dada uma relação $R$ sobre $A$, se tomarmos o fecho multiplicativo da relação de equivalência gerada por $R$, obteremos a menor congruência de $A$ que contém $R$. Essa congruência é a intersecção de todas as congruências de $A$ que contêm $R$ e será chamada de congruência gerada por $R$.

Uma relação $R$ em $A$ é antissimétrica se $x R y$ e $y R x$ implicam $x=y$. Uma relação antissimétrica, reflexiva e transitiva em $A$ é também dita uma ordem parcial em $A$ (ou simplesmente ordem em $A$ ).

Se temos uma ordem parcial $\leq$ fixada no conjunto $A$, dizemos que dois elementos $x$ e $y$ de $A$ são comparáveis se $x \leq y$ ou $y \leq x$. Como é de se esperar, diremos que $x$ e $y$ são incomparáveis se eles não forem comparáveis. Uma cadeia é um subconjunto $C$ de $A$ de modo que os elementos de $C$ são dois a dois comparáveis. Por outro lado, uma anticadeia é um subconjunto $X$ de $A$, de modo que os elementos de $X$ são dois a dois incomparáveis.

Dado um subconjunto $C$ de $A$, um elemento $x$ de $C$ é minimal (resp. maximal) se não existe $y \neq x$ em $C$ com $y \leq x$ (resp. $x \leq y$ ). Não é difícil ver que toda cadeia possui no máximo um elemento minimal (resp. maximal). Ademais, toda cadeia finita possui exatamente um elemento maximal e exatamente um elemento minimal.

Se $C$ é um subconjunto com um único elemento minimal (resp. maximal) $x$, então diremos que $x$ é o menor (resp. maior) elemento de $C$ ou que $x$ é o mínimo (resp. máximo) de $C$ e denotaremos esse fato por $x=\min (C)$ (resp. $x=\max (C)$ ).

Uma ordem parcial em $A$ é uma ordem total se todo par de elementos de $A$ for comparável. Claro que nesse caso, todo subconjunto de $A$ é uma cadeia.

Uma cadeia descendente infinita (em $A$ ) é uma seqüência infinita de elementos de $A$ da forma $\left(a_{i}\right)_{i \in \mathbb{N}}$, satisfazendo $a_{i+1}<a_{i}$ (utilizamos o símbolo $<$ para denotar que $a_{i+1} \leq a_{i}$ e $a_{i+1} \neq a_{i}$ ). Analogamente, uma cadeia ascendente infinita (em $A$ ) é uma seqüência infinita de elementos de $A$ da forma $\left(a_{i}\right)_{i \in \mathbb{N}}$, satisfazendo $a_{i+1}>a_{i}$. 
O seguinte conceito será utilizado exaustivamente em todo o trabalho.

Definição 1.1.1 (Boa Ordem) Uma ordem parcial $\leq$ em um conjunto A é uma boa ordem se todo subconjunto não vazio de A possui um menor elemento (segundo $\leq$ ).

Nesse caso, diremos que $A$ é bem ordenado (por $\leq$ ).

Exemplos de conjuntos bem ordenados são facilmente vistos na literatura. Desse modo, nos limitaremos a exemplificar esse conceito mencionando que o conjunto $\{i \in \mathbb{Z} \mid i \geq n\}$ (com a ordem canônica em $\mathbb{Z}$ ) é bem ordenado, para todo $n$ em $\mathbb{Z}$.

Suponha que temos uma boa ordem $\leq$ fixada em $A$. Então, para cada par $x, y$ de elementos de $A$ podemos considerar o subconjunto $\{x, y\}$. Assim, segue que toda boa ordem é uma ordem total. A seguinte proposição terá grande impacto em tudo que será desenvolvido neste trabalho. Ela pode ser facilmente verificada a partir da definição de boa ordem.

Proposição 1.1.1 Se uma ordem parcial $\leq$ em um conjunto A é uma boa ordem, então não existem cadeias descendentes infinitas em $A$. Em outras palavras, se $\leq$ é uma boa ordem então toda seqüência infinita de elementos de $A$ da forma:

$$
a_{1}>a_{2}>a_{3}>\cdots
$$

termina.

Demonstração. Mostraremos a afirmação acima em sua forma contrapositiva: se existe uma cadeia descendente infinita em $A$, então $\leq$ não é uma boa ordem.

Se $\left(a_{i}\right)_{i \in \mathbb{N}}$ é uma cadeia descendente infinita em $A$, então o conjunto $C:=\left\{a_{i} \mid i \in \mathbb{N}\right\}$ não possui um menor elemento. De fato, se $m \in C$ é o menor elemento de $C$, então $m=a_{j}$, para algum $j$. Mas então, como a cadeia é infinita, $a_{j+1}$ é estritamente menor que $m$, nos levando a uma contradição.

A propriedade expressa na proposição acima é comumente chamada de condição de cadeia descendente. De acordo com a proposição, ela é presente em conjuntos bem ordenados. Contudo, outras ordens (que não sejam boas ordens) podem apresentar essa propriedade. ${ }^{1}$

Aqui cabem alguns comentários sobre uma técnica de demonstração que usaremos recorrentemente neste trabalho. Esse tipo de argumentação é chamado por alguns autores de indução noetheriana.

Suponha que temos um conjunto $M$ bem ordenado por uma ordem $\leq$ e uma propriedade $P$, que cada elemento de $M$ pode ou não satisfazer. Então podemos provar a afirmação "cada elemento de $M$ satisfaz $P$ " da seguinte maneira. Assumimos por absurdo que a afirmação é falsa. Portanto, existem elementos de $M$ que não satisfazem $P$. Seja então

$$
C:=\{m \in M \mid m \text { não satisfaz } P\}
$$

Como $C$ é um subconjunto não vazio de $M$ e $M$ está bem ordenado, podemos tomar o menor elemento de $C$, segundo $\leq$; seja $a$ esse elemento. A contradição desejada pode então ser obtida

\footnotetext{
${ }^{1}$ As ordens que satisfazem a condição de cadeia descendente são freqüentemente chamadas de ordens bemfundamentadas (well-founded, no original). Tais ordens nāo precisam ser totais e, portanto, são uma generalização das boas ordens.
} 
se encontrarmos um elemento de $C$ menor que $a$. Ou ainda, pode-se chegar a essa contradição mostrando que $a$ satisfaz $P$. Para tanto, podemos utilizar o fato de que todo elemento menor que $a$ satisfaz $P$, pela minimalidade de $a$.

A técnica descrita acima será utilizada em muitos lugares. O leitor encontra alguns dos primeiros exemplos de sua utilização nas demonstrações dos Teoremas 2.4.2 e 3.1.6, do Lema 3.1.12 e da Proposição 3.2.1.

\subsection{Estruturas Algébricas}

Apresentamos aqui as principais estruturas algébricas que serão consideradas ao longo do texto. Procuramos exibir as principais definições e resultados relacionados a elas, mas estamos longe de poder dar toda a atenção que elas merecem. Assim, nos contentamos em apresentar apenas um panorama resumido e deixar que referências mais profundas [Jac85, Her64, Fra99, HK61, Lan71] preencham as lacunas deixadas por nós.

Semigrupos, Monóides e Grupos Uma operação binária em um conjunto $X$ é associativa se, para todos $x, y$ e $w$ em $X$, vale: $(x y) w=x(y w)$.

Um semigrupo é um par $(S, \cdot S)$, onde $S$ é um conjunto não vazio e $\cdot S$ uma operação binária associativa em $S$. Como usual, faremos um abuso de notação e denotaremos o semigrupo por $S$ e sua operação por ·, quando não houver dúvida sobre qual a operação em questão. ${ }^{2}$

O semigrupo $S$ é comutativo (ou abeliano) se, para todos $x$ e $y$ em $S$, vale: $x y=y x$. É comum denotar a operação de semigrupos comutativos aditivamente pelo símbolo $+_{S}$, em contraste com a notação multiplicativa usada até o momento. Dessa forma, a igualdade anterior ficaria $x+s y=y+s x$.

Dados dois subconjuntos $X$ e $Y$ do semigrupo $S$, podemos estender a operação $\cdot S$ definida entre elementos de $S$ para conjuntos de $S$. Assim, definimos o produto de dois conjuntos como sendo $X Y:=\{x \cdot s y \mid x \in X$ e $y \in Y\}$. Ao utilizar a notação aditiva, nos referiremos à soma de dois conjuntos: $X+_{S} Y:=\{x+s y \mid x \in X$ e $y \in Y\}$.

Dados dois semigrupos $S$ e $R$, um homomorfismo de semigrupos é uma função $\varphi: S \longrightarrow R$ que respeita a operação do semigrupo, isto é, para todos $x$ e $y$ em $S$, vale: $\varphi(x \cdot S y)=\varphi(x) \cdot R \varphi(y)$.

Um homomorfismo injetor será chamado de monomorfismo, um homomorfismo sobrejetor de epimorfismo e um homomorfismo bijetor de isomorfismo. Dizemos que dois semigrupos $S_{1}$ e $S_{2}$ são isomorfos se existe um isomorfismo entre eles e denotaremos esse fato por $S_{1} \cong S_{2}$. Um homomorfismo $\varphi: S \longrightarrow S$ é chamado de endomorfismo. Um endomorfismo que seja também um isomorfismo é chamado de automorfismo. Essa terminologia pode ser estendida facilmente para homomorfismos entre as estruturas algébricas abordadas adiante, de forma que a utilizaremos livremente também para esses homomorfismos.

Vale observar que, se tomarmos uma congruência $\equiv$ em um semigrupo $S$, o quociente $S / \equiv$ é um semigrupo. De fato, não é difícil verificar que $[a]_{\equiv} \cdot[b]_{\equiv} \subseteq\left[a \cdot_{S} b\right]_{\equiv}$, para todos $a$ e $b$ em $S$. Isso mostra que a multiplicação no quociente é independente de representante e, portanto, está bem definida.

Um conjunto gerador de um semigrupo $S$ é um subconjunto $G$ de $S$ de modo que todo elemento de $S$ se escreve como produto de elementos de $G$. Diremos então que $S$ é gerado por $G$.

\footnotetext{
${ }^{2}$ Essa abreviação será adotada também para as demais estruturas algébricas que definiremos.
} 
Ainda, se $S$ admitir algum conjunto gerador finito, diremos que $S$ é finitamente gerado (como semigrupo); caso contrário, diremos que é infinitamente gerado (como semigrupo).

Dado um semigrupo $S$, uma unidade de $S$ (também chamado de elemento neutro) é um elemento $1_{S}$ de $S$, com a seguinte propriedade: $1_{S} \cdot x=x \cdot 1_{S}=x$, para todo $x$ em $S$. É bem conhecido o fato de que, se $S$ possui uma unidade, então ela é única, de modo que podemos falar simplesmente na unidade de $S$ (caso ela exista). Ao utilizarmos a notação aditiva, denotaremos o elemento neutro por 0 .

Se temos um semigrupo multiplicativo $S$, podemos definir a noção de divisão em $S$. Diremos então que $u$ divide $v(u, v$ pertencem a $S)$ se:

- existem $a, b$ em $S$ tais que $v=a u b$ ou

- existe $a$ em $S$ tal que $v=a u$ ou $v=u a$.

Denotaremos o fato de que $u$ divide $v$ por $u \mid v$. Se $u \neq v$ e $u \neq 1$, diremos que $u$ é um divisor próprio de $v$. Por outro lado, se $u=v$ ou $u$ for o elemento neutro, diremos que $u$ é um divisor impróprio de $v$.

Ainda, se $v=u w$, diremos que $v$ é um múltiplo à esquerda (resp. à direita) de $w$ (resp. de $u$ ) e que $u$ (resp. $w$ ) é um divisor à esquerda (resp. à direita) de $v$, ou que $u$ (resp. $w$ ) é um prefixo (resp. sufixo) de $v$.

Um ideal de semigrupo é um subconjunto $I$ de um semigrupo $S$ com a seguinte propriedade: se $x$ pertence a $I$, então $a x$ e $x a$ também pertencem a $I$, para todo $a$ em $S$. Um conjunto gerador do ideal $I$ é um subconjunto $G$ de $I$, de modo que todo elemento de $I$ é divisível por (ou igual a) algum elemento de $G$. Caso $I$ admita algum conjunto gerador finito, diremos que $I$ é finitamente gerado. Se $I$ não for finitamente gerado, diremos que é infinitamente gerado.

Um monóide é uma tripla $\left(M, \cdot M, 1_{M}\right)$, de modo que $\left(M, \cdot_{M}\right)$ é um semigrupo e $1_{M}$ é o elemento neutro de $M$. Assim, um monóide nada mais é que um semigrupo munido de um elemento neutro. Um monóide é dito abeliano se for um semigrupo abeliano.

Dados dois monóides $M$ e $N$, um homomorfismo de monóides é um homomorfismo da forma $\varphi: M \longrightarrow N$ de semigrupos que satisfaz: $\varphi\left(1_{M}\right)=1_{N}$. $\left.1_{N}\right\}$.

Denotaremos por $\operatorname{Ker}(\varphi)$ o kernel (ou núcleo) da função $\varphi$, isto é, $\operatorname{Ker}(\varphi):=\{m \in M \mid \varphi(m)=$

Dado um monóide $M$, um elemento $m$ de $M$ é inversível se existe $m^{\prime}$ em $M$, de modo que $m m^{\prime}=m^{\prime} m=1$. Um grupo é um monóide em que todo elemento é inversível. Um grupo é abeliano se for um monóide abeliano.

Se tomarmos uma congruência $\equiv$ em um monóide $M$, o quociente de $M$ por $\equiv$ é um monóide (com unidade $\left[1_{M}\right]_{\equiv}$ ).

Anéis Um anel é uma quíntupla $(A,+, \cdot, 0,1)$, satisfazendo:

- $(A,+, 0)$ é um grupo abeliano (que denotaremos com a notação aditiva).

- $(A, \cdot 1)$ é um monóide.

- Para todos $a, b$ e $c$ em $A$, valem: 


$$
\begin{aligned}
& a \cdot(b+c)=(a \cdot b)+(a \cdot c) . \\
& (b+c) \cdot a=(b \cdot a)+(c \cdot a) .
\end{aligned}
$$

É usual encontrarmos definições mais gerais de anéis - que não exigem a presença de unidade multiplicativa ou mesmo da associatividade da multiplicação. Contudo, ao longo de todo o trabalho, assumiremos que todos os anéis são associativos e possuem unidade. Dessa forma, preferimos a versão acima.

Um anel como definido acima é abeliano se $(A, \cdot, 1)$ for um monóide comutativo.

Dados dois anéis $A$ e $B$, um homomorfismo de anéis é uma aplicação $\varphi: A \longrightarrow B$ que satisfaz:

- $\varphi\left(a+{ }_{A} b\right)=\varphi(a)+{ }_{B} \varphi(b)$.

- $\varphi\left(a \cdot{ }_{A} b\right)=\varphi(a) \cdot B \varphi(b)$.

- $\varphi\left(1_{A}\right)=1_{B}$.

Em outras palavras, $\varphi$ é um homomorfismo de monóides com respeito à estrutura aditiva e multiplicativa do anel $A$. Note que a terceira propriedade listada na definição não é conseqüência das duas primeiras.

Vale ressaltar que muitas vezes na literatura, a definição de homomorfismo de anéis não exige que a imagem da unidade do domínio seja a unidade do contra-domínio. Homomorfismos que possuem essa última propriedade são chamados então de homomorfismos de anéis com unidade. Contudo, uma vez que trabalharemos somente com anéis com unidade preferiremos a definição ora apresentada. $\left.0_{B}\right\}$.

Denotaremos por $\operatorname{Ker}(\varphi)$ o kernel (ou núcleo) da função $\varphi$, isto é, $\operatorname{Ker}(\varphi):=\{a \in A \mid \varphi(a)=$

Dado um anel $A$, um ideal (de anel) de $A$ é um subconjunto $I$ de $A$ com a seguintes propriedades:

- Se $a$ e $b$ pertencem a $I$, então $a+b$ pertence a $I$.

- Se $a$ pertence a $I$ e $c$ pertence a $A$, então $a \cdot c$ e $c \cdot a$ pertencem a $I$.

O leitor familiarizado com as noções de ideais à esquerda e à direita deve notar que nossos ideais serão sempre bilaterais (como explícito na definição acima).

É um fato bem conhecido que, se $\varphi$ é um homomorfismo de anéis de $A$ em $B$, então $\operatorname{Ker}(\varphi)$ é um ideal de anel de $A$

Dado um conjunto $G:=\left\{g_{1}, g_{2}, \ldots\right\}$ (não necessariamente finito) do anel $A$, podemos considerar a intersecção de todos os ideais de $A$ que contêm $G$. Essa intersecção é um ideal $I$ de $A$ e seus elementos são da forma:

$$
\sum_{i=1}^{k} \sum_{j=1}^{m_{i}} a_{i, j} g_{i} a_{i . j}^{\prime}
$$

com $a_{i, j}$ e $a_{i, j}^{\prime}$ em $A$, para todos $i, j$. Chamamos $I$ de o ideal gerado por $G$ e denotamos esse fato por $I=\langle G\rangle$. Ressaltamos que a notação $\langle G\rangle$ denotará sempre o ideal de anel (ou de 
álgebra, como será visto adiante) gerado pelo conjunto $G$. Conforme necessário, outras notações serão introduzidas para denotar ideais de semigrupo.

Um dos pontos fundamentais de ideais é que eles definem congruências no anel. De fato, dado um ideal $I$ do anel $A$, podemos definir que $a \sim b$ se e somente se $a-b$ pertence a $I$. Com isso, pode-se verificar de maneira bem natural que $\sim$ é uma congruência em $A$. Ainda, cada classe de $A / \sim$ é da forma

$$
[a]_{\sim}=\{a+i \mid i \in I\}
$$

de modo que denotaremos a classe do elemento $a$ de $A$ por $a+I$. Ainda, podemos introduzir uma estrutura de anel no quociente $A / \sim$ por meio das operações:

$$
\begin{aligned}
& (a+I)+(b+I):=(a+b)+I \\
& (a+I) \cdot(b+I):=(a \cdot b)+I
\end{aligned}
$$

Não é difícil verificar que as operações dadas acima estão bem definidas. Com isso, temos uma estrutura de anel no quociente de $A$ por $\sim$. Chamaremos esse anel de anel quociente e o denotaremos por $A / I$.

Dados $A$ e um ideal $I$ de $A$, a projeção canônica de $A$ em $A / I$ é a aplicação $\pi$ dada por:

$$
\begin{aligned}
\pi: & A \longrightarrow A / I \\
& a \mapsto a+I
\end{aligned}
$$

Novamente, é bastante rotineira a demonstração de que a aplicação $\pi$ está bem definida e que se trata de um epimorfismo de anéis. Note que $\pi(a)=0$ se e somente se $a$ pertence a $I$ e que $\pi(a)=\pi(b)$ se e somente se $a-b$ pertence a $I$.

Um anel que terá destaque neste texto (em especial no Capítulo 2) é o anel de polinômios comutativo em várias variáveis: $K\left[x_{1}, \ldots, x_{n}\right]$. É interessante notar que, além da estrutura de anel, $K\left[x_{1}, \ldots, x_{n}\right]$ possui uma estrutura de espaço vetorial sobre $K$ (com os monômios formando uma base).

O seguinte resultado é bastante famoso e sua demonstração pode ser vista em muitos textos clássicos de álgebra.

Proposição 1.2.1 (Primeiro Teorema do Homomorfismo) Sejam A e B anéis e seja

$$
\varphi: A \longrightarrow B
$$

um epimorfismo. Então $A / \operatorname{Ker}(\varphi)$ é isomorfo a $B$.

Dado um conjunto $X$ qualquer, é bem conhecido o fato de que a relação de inclusão de subconjuntos forma uma ordem parcial em $\mathcal{P}(X)$. Assim, dizemos que um anel $A$ é noetheriano se não existem cadeias ascendentes infinitas de ideais de $A$ (com a relação de inclusão de subconjuntos). Em outras palavras, $A$ é noetheriano se toda seqüència da forma

$$
I_{0} \subsetneq I_{1} \subsetneq I_{2} \cdots
$$

(com $I_{i}$ um ideal de $A$, para todo $i \geq 0$ ) termina. 
Lembramos o leitor que um domínio de integridade é um anel com a seguinte propriedade: se $a b=0$, então $a=0$ ou $b=0$.

Um corpo é um anel $(K,+, \cdot, 0,1)$, de modo que $(K, \cdot, 1)$ é um grupo abeliano. É bem conhecido o fato de que todo corpo é um domínio de integridade.

Espaços Vetoriais Assumiremos familiaridade do leitor com os conceitos básicos de álgebra linear. Notamos somente que faremos uso de espaços vetoriais infinitamente gerados. Exposições detalhadas sobre eles podem ser vistas em muitos textos clássicos [HK61, Lan71] e no Capítulo 3 de [BW93].

Seja $V$ um espaço vetorial e $X$ uma base de $V$. Dado um elemento $f$ em $V$, é claro que $f$ se escreve de maneira única como combinação linear de elementos de $X$ :

$$
f=\sum_{i=1}^{n} \alpha_{i} x_{i}
$$

(assumimos que $x_{h} \neq x_{j}$ se $h \neq j$ ).

Definimos então o suporte de $f$ (com relação à base $X$ ) como sendo o conjunto formado pelos elementos de $X$ que aparecem com coeficiente não nulo na expressão de $f$ como combinação linear de elementos de $X$. Mais precisamente, temos:

$$
\operatorname{supp}(f):=\left\{x_{i} \mid 1 \leq i \leq n \text { e } \alpha_{i} \neq 0\right\}
$$

Convencionamos que o suporte do vetor nulo é o conjunto vazio. $W$.

Ainda, dado um subconjunto $W$ de $V$, denotaremos por $\operatorname{span}(\mathrm{W})$ o espaço vetorial gerado por

Álgebras As álgebras serão talvez a estrutura algébrica abstrata mais presente neste trabalho. Suas principais propriedades podem ser vistas em profundidade em muitos textos clássicos [Jac85, Pie82, CLS83], de forma que apresentaremos aqui apenas e definição e o mínimo de conceitos necessário ao restante da dissertação.

Dado um corpo $K$, um anel $A$ é dito uma $K$-álgebra (ou simplesmente uma álgebra, quando não houver dúvidas quanto ao corpo em questão) se for um anel e um $K$-espaço vetorial, satisfazendo:

$$
\alpha(a b)=(\alpha a) b=a(\alpha b)
$$

para todos $a$ e $b$ em $A$ e $\alpha$ em $K$.

Consideraremos sempre o corpo $K$ como um subanel da $K$-álgebra $A$ mediante o homomorfismo de anéis que associa cada elemento $\alpha$ em $K$ ao elemento $\alpha 1$ de $A$. Com isso, cada elemento de $K$ comuta com todos os elementos de $A$ :

$$
\alpha a=\alpha(a 1)=(\alpha a) 1=a(\alpha 1)=a \alpha
$$

Diremos que uma $K$-álgebra $A$ tem dimensão finita se $A$ for um espaço vetorial de dimensão finita sobre $K$.

Exemplos bastante famosos de álgebras são dados pelos anéis de polinômios. Em particular, podemos considerar o anel de polinômios comutativo em $n$ variáveis, $K\left[x_{1}, \ldots, x_{n}\right]$. É claro que essa álgebra possui dimensão infinita. 
Outro exemplo de álgebra que será bastante interessante para nós é o anel de polinômios não comutativo em várias variáveis: $K\left\langle x_{1}, x_{2}, \ldots, x_{n}\right\rangle$. Referiremo-nos a ele como álgebra livre e exploraremos melhor algumas de suas propriedades adiante. Note que tal qual o anel de polinômios comutativo, a álgebra livre também possui dimensão infinita. Um terceiro exemplo são as álgebras de caminhos, que serão vistas na Seção 1.4.

O produto direto de duas $K$-álgebras $A_{1}$ e $A_{2}$ é a álgebra composta pelo conjunto $A_{1} \times A_{2}$ e com as operações feitas componente a componente. Diremos então que uma álgebra $A$ é decomponível se ela for isomorfa ao produto direto de duas álgebras. Como é de se esperar, diremos que $A$ é indecomponivel se $A$ não for decomponível.

Um conjunto gerador de $A$ como álgebra é um subconjunto $G$ de $A$, de modo que todo elemento de $A$ se escreve como combinação linear de produtos de elementos de $G$. Mais precisamente, todo elemento $a$ de $A$ é da forma:

$$
a=\sum_{i=1}^{k} \lambda_{i} f_{i_{1}} f_{i_{2}} \cdots f_{i_{n(i)}}
$$

com $\lambda_{i}$ em $K$, para todo $1 \leq i \leq k, f_{i_{h}}$ em $G$, para todo $h$.

Diremos que $A$ é finitamente gerada (como álgebra) se possuir um conjunto gerador finito (como álgebra). Caso contrário, diremos que $A$ é infinitamente gerada (como álgebra). Note que tanto $K\left[x_{1}, \ldots, x_{n}\right]$, quanto $K\left\langle x_{1}, x_{2}, \ldots, x_{n}\right\rangle$ são álgebras finitamente geradas. Em ambos os casos, as variáveis (vistas como monômios da álgebra) constituem um conjunto gerador finito.

Dadas duas álgebras $A_{1}$ e $A_{2}$ sobre $K$, uma aplicação $\varphi: A_{1} \longrightarrow A_{2}$ é um homomorfismo de álgebras se for uma aplicação linear e um homomorfismo de anéis. Não é difícil ver que se dois homomorfismos de álgebras $\varphi: A_{1} \longrightarrow A_{2}$ e $\alpha: A_{1} \longrightarrow A_{2}$ coincidem em um conjunto gerador $G$ de $A_{1}$ (como álgebra), então eles são iguais.

Um ideal (de álgebra) de $A$ é um subconjunto $I$ de $A$ que é um ideal de anel e um subespaço vetorial de $A$. Note que se $I$ satisfizer as exigências da definição de ideal de anel (ser fechado por soma e por multiplicação de um elemento qualquer de $A$ ), então ele será automaticamente um espaço vetorial. Esse fato é facilmente demonstrado por meio da definição de álgebra: se $\lambda$ é um escalar e $g$ um elemento de um ideal $I$ de anel de $A$, então

$$
\lambda g=\lambda(1 g)=\underbrace{(\lambda 1)}_{\in A} g \in I
$$

Assim, dado um subconjunto $I$ de $A$, para mostrarmos que se trata de um ideal de álgebra de $A$, basta mostrar que ele é um ideal de anel.

Um conjunto gerador de um ideal $I$ de $A$ é um subconjunto $G$ de $I$ com a propriedade de que todo elemento de $I$ se escreve como combinação linear de múltiplos de elementos de $G$. Mais precisamente, temos que todo elemento $f$ de $I$ é da forma:

$$
f=\sum_{i=1}^{n} \lambda_{i} u_{i} g_{j_{i}} v_{i}
$$

com $\lambda_{i}$ escalares, $u_{i}, v_{i}$ elementos de $A$ e $g_{j_{i}}$ em $G$, para todo $1 \leq i \leq n$.

Podemos então agrupar os somandos com mesmo $g_{j_{i}}$ da igualdade acima para obter: 


$$
f=\sum_{h=1}^{n} \sum_{i=1}^{n_{h}} \lambda_{h, i} u_{h, i} g_{j_{h}} v_{h, i}
$$

Por outro lado, se temos uma base $\mathcal{B}$ de $A$ fixada, é claro que os elementos $u_{h, i}$ e $v_{h, i}$ se escrevem como combinação linear de elementos de $\mathcal{B}$. Assim, podemos aplicar as leias distributivas para obter:

$$
f=\sum_{h=1}^{m} \sum_{i=1}^{m_{h}} \beta_{h, i} b_{h, i} g_{l_{h}} b_{h, i}^{\prime}
$$

com $\beta_{h, i}$ escalares, $b_{h, i}, b_{h, i}^{\prime}$ elementos de $\mathcal{B}$ e $g_{l_{h}}$ em $G$, para todos $h, i$.

Expressões da forma da Equação 1.1 serão bastante utilizadas ao longo de todo o texto quando lidarmos com conjuntos geradores de ideais.

Diremos que um ideal $I$ é finitamente gerado se possuir algum conjunto gerador finito. Caso contrário, diremos que $I$ é infinitamente gerado.

Se $I$ é um ideal da $K$-álgebra $A$, então o quociente (de anel) $A / I$ é novamente uma álgebra sobre $K$. Em particular, ela possui a estrutura do espaço quociente $A / I$.

Monóides e Álgebras Livres Chamaremos de alfabeto um conjunto finito de símbolos. Dado um alfabeto, chamaremos seus elementos de letras.

Se temos um alfabeto $X$, podemos tomar seqüências de elementos de $X$ :

$$
\left(x_{1}, x_{2}, \ldots, x_{r}\right)
$$

$\operatorname{com} x_{i}$ em $X$, para $1 \leq i \leq r$.

Chamaremos essas seqüências de palavras (sobre $X$ ) e um conjunto de palavras (sobre $X$ ) será chamado de linguagem (sobre $X$ ).

Se temos duas palavras, podemos definir seu produto por concatenação. Assim, se tomamos duas palavras $s:=\left(x_{1}, x_{2}, \ldots, x_{r}\right)$ e $r:=\left(y_{1}, y_{2}, \ldots, y_{l}\right)$, teremos:

$$
s r=\left(x_{1}, x_{2}, \ldots, x_{r}, y_{1}, y_{2}, \ldots, y_{l}\right)
$$

É imediato ver que essa operação é associativa. Ademais, se tomarmos a seqüência vazia ( ), ela pode ser vista como uma palavra sobre $X$ que não contém letras. Ainda, não é difícil ver que ela é uma unidade do conjunto de todas as palavras sobre $X$ com a operação de concatenação.

Portanto, o conjunto de todas as palavras sobre $X$, munido da operação de concatenação, forma um monóide. Chamaremos esse monóide de monóide livre sobre $X$ e o denotaremos por $X^{*}$. Vale ressaltar que, uma vez que definimos alfabetos como conjuntos finitos, nossos monóides livres serão sempre finitamente gerados.

Denotaremos ainda uma seqüência $\left(x_{i}\right)$ simplesmente por $x_{i}$. Com isso, pode-se denotar um elemento $\left(x_{1}, x_{2}, \ldots, x_{r}\right)$ de $X^{*}$ por $x_{1} x_{2} \ldots x_{r}$.

Algumas vezes, será conveniente considerar o monóide livre $X^{*}$ sem a palavra vazia e passar a vêlo como um semigrupo. Chamaremos esse semigrupo de semigrupo livre sobre $X$ e o denotaremos por $X^{+}$. Com isso, $X^{+}:=X^{*} \backslash\{1\}$. 
Dada uma palavra $u$ sobre $X$ definimos seu comprimento (ou grau) como o número de letras que a compõem e o denotaremos por $\|u\|$. A palavra vazia é a única palavra de $X^{*}$ com comprimento zero e é fácil ver que $\|u v\|=\|u\|+\|v\|$. Ainda, se $y$ é uma letra, denotaremos por $\|u\|_{y}$ o número de ocorrências de $y$ na palavra $u$.

Mencionamos em seguida alguns resultados importantes envolvendo monóides livres. Não apresentaremos demonstrações para as afirmações feitas aqui, uma vez que essas podem ser vistas em muitos textos que abordam esses monóides [Ber79, $\left.\mathrm{LSS}^{+} 79\right]$.

Uma propriedade importante do semigrupo livre $X^{+}$(e também do monóide livre $X^{*}$ ) é que todo elemento se escreve de maneira única como produto de letras em $X$.

Consideremos $X$ e $\Sigma$ dois alfabetos e $\varphi: X^{*} \longrightarrow \Sigma^{*}$ e $\alpha: X^{*} \longrightarrow \Sigma^{*}$ dois homomorfismos de monóides. É bem conhecido o fato de que se $\varphi$ e $\alpha$ coincidem nos elementos de $X$, então eles são iguais. Ainda, se $M$ é um monóide qualquer e $\beta: X \longrightarrow M$ uma função, então existe uma única extensão de $\beta$ a um homomorfismo de monóides de $X^{*}$ em $M$.

Seja $K$ um corpo, $X$ um alfabeto e considere o monóide livre sobre $X$. Se tomarmos o conjunto composto por todas as combinações lineares sobre $K$ de elementos de $X^{*}$, teremos um espaço vetorial sobre $K$. Ainda, podemos estender a estrutura multiplicativa de $X^{*}$ linearmente para essas combinações. Com isso, obtemos uma álgebra, chamada de álgebra livre sobre $X$ e denotada por $K\langle X\rangle$.

Outra forma de ver a álgebra livre é pensá-la como o anel de polinômios não comutativo, com os símbolos de $X$ no papel das variáveis.

Note que $K\langle X\rangle$ é finitamente gerada como álgebra ( $X$ é um conjunto gerador), mas possui dimensão infinita. Freqüentemente, ao trabalharmos com a álgebra livre, consideraremos sua base composta por todos os elementos de $X^{*}$.

\subsection{Grafos}

Apresentamos nesta seção alguns conceitos fundamentais relacionados à Teoria dos Grafos. Vale ressaltar que alguns dos conceitos aqui apresentados podem diferir ligeiramente daqueles comumente vistos na literatura. Entretanto, essas diferenças serão bastante pequenas e só serão introduzidas com o intuito de fornecer maior comodidade ao lidar com o uso particular desses conceitos neste trabalho. Algumas referências interessantes sobre grafos são [BM76, CLRS01, Feo01]

O leitor familiarizado com o uso de álgebras de caminhos em Teoria de Representações de Álgebras (e em outras áreas da Matemática) deve notar que se trata exatamente do conceito de carcaz. Entretanto, optamos pela terminologia apresentada aqui por ela ser clássica em Ciência da Computação.

Um grafo (dirigido) é um par $\Gamma=(V, A)$, onde $V$ é um conjunto arbitrário chamado conjunto de vértices e $A$ é um conjunto arbitrário chamado de conjunto de arcos. Cada arco está associado a dois vértices: à sua ponta inicial, ou origem, e à sua ponta final, ou término.

Mais formalmente, podemos ver $\Gamma$ como uma tripla $\Gamma=(V, A, f)$, onde $f$ é uma função de $A$ em $V \times V$ que associa cada arco a sua origem e a seu término.

Dizemos que o grafo $\Gamma$ é finito se os conjuntos $V$ e $A$ forem finitos. No restante de todo o trabalho, assumiremos que nossos grafos são finitos, exceto quando explicitado de forma contrária.

Deve estar claro que podem existir arcos que possuem o mesmo vértice como ponta inicial e ponta final. Tais arcos serão chamados de laços. Ainda, são permitidos arcos paralelos em nossos grafos, ou seja, arcos cuja origem e término coincidem. 
Um exemplo de grafo (em sua representação pictórica tradicional) pode ser visto na Figura 1.1.

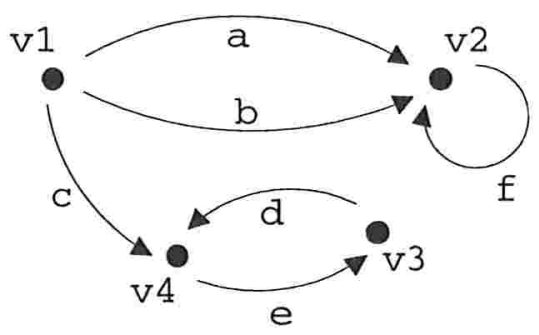

Figura 1.1: Grafo $H$

Dado um grafo $\Gamma$ qualquer, denotaremos seu conjunto de vértices por $V(\Gamma)$ e seu conjunto de arcos por $A(\Gamma)$. Na Figura 1.1, $A(H)=\{a, b, c, d, e, f\}$ e $V(H)=\left\{v_{1}, v_{2}, v_{3}, v_{4}\right\}$. Ainda, $a$ e $b$ são arcos paralelos e $f$ é um laço.

Um caminho (ou passeio) em um grafo $\Gamma$ é uma seqüência da forma

$$
c=v_{0} a_{1} v_{1} a_{2} v_{2} \cdots v_{n-1} a_{n} v_{n}
$$

onde $v_{j}$ pertence a $V(\Gamma)$, para todo $0 \leq j \leq n$ e $a_{i}$ é um arco de $\Gamma$ com origem $v_{i-1}$ e término $v_{i}$, para todo $1 \leq i \leq n .^{3}$ Diremos que a origem do caminho $c$ é $v_{0}$; ou ainda que $c$ começa em $v_{0}$. Analogamente, o término do caminho $c$ é $v_{n}$ e diremos que $c$ termina em $v_{n}$.

Dois caminhos distintos são ditos paralelos se eles possuem a mesma origem e o mesmo término.

O comprimento (ou grau) de um caminho é o número de arcos que existe nele. Assim, o comprimento de $c$ é $n$. Dado um caminho $c$, denotaremos seu comprimento por $\|c\|$. Com isso, arcos podem ser vistos como caminhos de comprimento 1 . Vértices serão vistos como caminhos de comprimento 0 e chamados de caminhos triviais. Retomando o grafo $H$ da Figura 1.1, temos alguns exemplos de caminhos:

$$
\begin{array}{ll}
c_{1}:=v_{1} a v_{2} f v_{2} f v_{2} & c_{2}:=v_{1} c v_{4} e v_{3} d v_{4} \\
c_{3}:=v_{3} & c_{4}:=v_{1} c v_{4}
\end{array}
$$

Note que $\left\|c_{1}\right\|=\left\|c_{2}\right\|=3,\left\|c_{3}\right\|=0$ e $\left\|c_{4}\right\|=1$.

Muitas vezes será conveniente denotar um caminho $c$ simplesmente por sua seqüência de arcos. Assim, $c$ pode ser denotado simplesmente por $c=a_{1} a_{2} \cdots a_{n}$. Um caminho de comprimento zero será denotado pelo vértice que o compõe. Note que essa representação determina unicamente um caminho. $^{4}$

Com isso, podemos denotar os caminhos $c_{1}, c_{2}$ e $c_{3}$ vistos antes por aff, ced e $v_{3}$, respectivamente.

Diremos que um subcaminho $c^{\prime}$ de $c=a_{1} a_{2} \cdots a_{n}$ é uma subsequiência de seus arcos. Assim, $c^{\prime}=a_{i} a_{i+1} \cdots a_{r}$, com $1 \leq i \leq r \leq n$. Se $c$ for um caminho trivial, seu único subcaminho será ele próprio.

\footnotetext{
${ }^{3}$ É comum diferenciar a noção de passeio da de caminho em grafos dirigidos. Contudo, não faremos essa distinção aqui por ela não ser necessária em nosso uso de grafos. Ademais, a noção de "caminho" presente nas álgebras de caminhos é exatamente a definida aqui.

${ }^{4} \mathrm{Na}$ literatura, é comum denotar um caminho por sua seqüência de vértices (note que, quando são permitidos arcos paralelos, uma seqüència de vértices pode denotar mais de um caminho). Entretanto, a notação fixada aqui é mais apropriada para nossos propósitos.
} 
Dados dois caminhos $c=a_{1} a_{2} \cdots a_{n}$ e $c^{\prime}=b_{1} b_{2} \cdots b_{r}$ tais que a origem de $c$ é igual ao término de $c^{\prime}$, a concatenação de $c^{\prime} \operatorname{com} c$ é o caminho

$$
c^{\prime} c:=b_{1} b_{2} \cdots b_{r} a_{1} a_{2} \cdots a_{n}
$$

Se $c$ e $c^{\prime}$ forem como acima e $c^{\prime}$ (resp. $c$ ) for um caminho de comprimento nulo, então $c^{\prime} c$ é definido como $c^{\prime} c:=c$ (resp. $c^{\prime} c:=c^{\prime}$ ). Note que o comprimento da concatenação de dois caminhos é a soma de seus comprimentos.

Um ciclo é um caminho que começa e termina no mesmo vértice. Ainda, se um ciclo não tiver vértices repetidos (excetuando-se sua origem e seu término, naturalmente), diremos que ele é um ciclo simples. Um grafo é dito acíclico se ele não possuir ciclos.

Seja $\Gamma$ um grafo e tomemos um subconjunto $X$ de $V(\Gamma)$. Chamaremos de subgrafo determinado por $X$ o grafo $\Gamma^{\prime}$ que contém como conjunto de vértices $X$ e como conjunto de arcos todos os arcos de $\Gamma$ com ponta inicial e final em elementos de $X$.

Diremos simplesmente que $\Gamma^{\prime}$ é um subgrafo de $\Gamma$ para denotar o fato de que $\Gamma^{\prime}$ é o subgrafo de $\Gamma$ determinado por $X$, para algum subconjunto $X$ de $V(\Gamma)$. Ainda, $\Gamma^{\prime}$ é dito um subgrafo próprio de $\Gamma$ se $X$ for um subconjunto próprio de $V(\Gamma)$.

Dado um grafo $\Gamma$, um subgrafo $\Gamma^{\prime}$ de $\Gamma$ é chamado de convexo (em $\Gamma$ ) se todo arco de $\Gamma$, com ponta inicial ou final em $V\left(\Gamma^{\prime}\right)$ é um arco de $\Gamma^{\prime}$.

Dado um grafo $\Gamma$, uma componente de $\Gamma$ é um subgrafo convexo $\Gamma^{\prime}$ que não possui subgrafos convexos próprios.

Aqui talvez seja o caso de explorar informalmente o que significa dizer que a única componente de $\Gamma$ é $\Gamma$. Esse significado será utilizado na seção seguinte. Se $\Gamma$ satisfaz essa exigência, então um subgrafo próprio qualquer $\Gamma^{\prime}$ de $\Gamma$ não é convexo. Logo, $\Gamma^{\prime}$ tem a seguinte propriedade: para algum vértice $v_{1}$ de $\Gamma^{\prime}$, existe um vértice $v_{2}$ em $V(\Gamma) \backslash V\left(\Gamma^{\prime}\right)$ ligado a $v_{1}$ por um arco de $\Gamma$.

\section{4 Álgebras de Caminho}

Introduzimos aqui uma das estruturas algébricas mais presentes ao longo do texto. Essas álgebras são muito estudadas em Teoria de Representações de Álgebras e possuem uma importância central nessa teoria e em outras áreas da Álgebra [ARS95, CLS83]. Conforme veremos adiante, elas também têm um papel fundamental em Teoria de Bases de Gröbner não comutativas.

Seja $K$ um corpo e $\Gamma$ um grafo. A partir deles definiremos uma álgebra $K \Gamma$. Primeiramente, consideremos $\mathcal{P}$ o conjunto de todos os caminhos de $\Gamma$. Lembramos que os vértices também estão incluídos em $\mathcal{P}$ através dos caminhos de comprimento zero.

Considere $K \Gamma$ como sendo o $K$-espaço vetorial com base $\mathcal{P}$. Para transformarmos $K \Gamma$ numa álgebra, precisamos definir uma multiplicação em $\mathcal{P}$. O produto em $\mathcal{P}$ será definido por concatenação de caminhos de $\Gamma$ da seguinte forma. Sejam $a$ e $c$ elementos de $\mathcal{P}$. Então:

$$
a \cdot c:=\left\{\begin{array}{l}
\text { o caminho } a c, \text { se o término de } a \text { for igual à origem de } c ; \\
0, \text { caso contrário. }
\end{array}\right.
$$

Estendendo por linearidade essa multiplicação a todos os elementos de $K \Gamma$, temos uma estrutura de álgebra bem definida. A álgebra $K \Gamma$ definida acima é chamada de álgebra de caminhos.

Consideraremos sempre como uma base distinguida de $K \Gamma$ a base $\mathcal{P}$ composta por todos os caminhos em $\Gamma$. 
Antes de mais nada, note que $K \Gamma$ é uma álgebra de dimensão finita se e somente se $\Gamma$ for um grafo acíclico. Ademais, ela é uma álgebra finitamente gerada se $\Gamma$ for um grafo finito: o conjunto $A(\Gamma) \cup V(\Gamma)$ constitui um conjunto gerador de $K \Gamma$ como álgebra.

Outra propriedade importante dessas álgebras e que enunciaremos sem demonstração é o fato de que $K \Gamma$ é uma álgebra indecomponível se e somente se a única componente de $\Gamma$ é $\Gamma$. Uma demonstração para esse fato pode ser vista no Capítulo 2 de [CLS83].

Podemos ilustrar a estrutura algébrica das álgebras de caminhos se tomarmos o grafo $H$ visto como exemplo na Figura 1.1 e considerarmos a álgebra de caminhos $\mathbb{Q} H$ :

São elementos de $\mathbb{Q} H$ :

$$
\begin{array}{ll}
f_{1}:=3 c e d+25 a f^{2} & f_{2}:=2 e d e+3 f^{2} \\
f_{3}:=c(e d)^{2} & f_{4}:=v_{4} \\
f_{5}:=e & f_{6}:=b
\end{array}
$$

Então:

$$
\begin{array}{ll}
f_{4} f_{6}=f_{4} f_{3}=0 & f_{4}^{2}=f_{4} \\
f_{4} f_{5}=f_{5} & f_{3} f_{5}=c(e d)^{2} e \\
f_{5}^{2}=0 & f_{5} f_{6}=f_{6} f_{5}=f_{3} f_{6}=f_{6} f_{3}=0 \\
f_{1} f_{5}=3 \mathrm{ced} \cdot e+25 a f^{2} \cdot e=3 \mathrm{cede} & f_{5} f_{2}=2 e \cdot e d e+3 e \cdot f^{2}=0
\end{array}
$$

e

$$
f_{1} f_{2}=6 c e d \cdot e d e+9 c e d \cdot f^{2}+50 a f^{2} \cdot e d e+75 a f^{2} \cdot f^{2}=6 c e d e d e
$$

Não é difícil ver que $\mathcal{P} \cup\{0\}$ forma um semigrupo e é interessante ressaltar que não se trata de um monóide - a unidade de $K \Gamma$ não pertence a $\mathcal{P}$ em geral, conforme discutiremos adiante. Essa observação nos será valiosa adiante.

Considere o grafo $H$ da Figura 1.1 e tome a álgebra $\mathbb{Q} H$. Note que $c:=v_{1} \cdot a \cdot v_{2}=v_{1} \cdot a=a$. Com isso, temos mais de uma maneira de escrever o caminho $c$.

Assim, nos referiremos sempre à forma canônica de um elemento $c$ de $\mathcal{P}$ para indicar a seqüência de arcos que compõem $c$ (ou o vértice que o compõe, no caso de o comprimento de $c$ ser nulo). É claro que, para todo elemento $c$ de $\mathcal{P}$, existe uma única forma canônica para $c$ : é exatamente a seqüência de arcos (ou o vértice) que determina o caminho em $\Gamma$ que esse elemento representa.

Não é difícil ver que em uma álgebra de caminhos $K \Gamma$, os vértices são idempotentes, isto é, para cada vértice $v$ em $\mathcal{P}, v^{2}=v$. Ademais, é claro pela definição de concatenação de caminhos, que os vértices são os únicos idempotentes em $\mathcal{P}$ (basta notar que, se $c$ é um caminho de comprimento maior que 0 e $c^{2} \neq 0$, então $\left.\left\|c^{2}\right\|>\|c\|\right)$.

Ademais, o conjunto de todos os vértices de $\Gamma$ é um conjunto ortogonal, ou seja $v_{i} v_{j}=0$, para todo par de elementos distintos $v_{i}, v_{j}$ de $V(\Gamma)$.

Assim, se tomarmos um caminho $c$ e um vértice $v, c v$ (resp. $v c$ ) é não nulo se e somente se $v$ for o término (resp. a origem) de $c$. Essa afirmação é conseqüência imediata da ortogonalidade de $V(\Gamma)$ e da observação simples de que, se denotamos por $o$ e $t$ a origem e o término de $c$, respectivamente, então $c=o \cdot c \cdot t$. Portanto,

$$
c=\left(\sum_{v \in V(\Gamma)} v\right) c=c\left(\sum_{v \in V(\Gamma)} v\right)
$$


Isso nos permite concluir o importante fato de que, em uma álgebra de caminhos $K \Gamma$, vale a seguinte igualdade:

$$
1=\sum_{v \in V(\Gamma)} v
$$

Dada uma álgebra de caminhos $K \Gamma$, denotaremos por $J_{K \Gamma}$ o ideal de álgebra de $K \Gamma$ gerado pelos arcos de $\Gamma$. Quando o contexto tornar claro qual a álgebra de caminhos em questão, denotaremos esse ideal simplesmente por $J$.

Será bastante comum lidarmos com potências desse ideal; e não é difícil ver que $J^{n}$ é um ideal, para todo $n>0$. Informalmente, $J^{n}$ é o ideal gerado por todos os caminhos de comprimento maior ou igual a $n$, ou seja, $J^{n}$ contém todas as combinações lineares de caminhos de comprimento maior ou igual a $n$.

Um ideal de $K \Gamma$ é dito homogêneo se existe um conjunto gerador $F$ de $I$ de modo que todo elemento $f$ de $F$ satisfaz a seguinte propriedade: existe um inteiro $n$ (que depende de $f$ ) tal que, para todo $b$ em $\operatorname{supp}(f),\|b\|=n$.

Exploramos a seguir a relação das álgebras de caminhos com as álgebras livres. Na realidade, veremos que toda álgebra livre é isomorfa a um tipo especial de álgebra de caminhos. Dessa maneira, podemos ver as álgebras livres como um caso particular das álgebras de caminhos.

Seja $K\langle X\rangle$ uma álgebra livre, de modo que $X=\left\{x_{1}, x_{2}, \ldots, x_{n}\right\}$. Construímos então o seguinte grafo $\Gamma: V(\Gamma):=\{v\} ; A(\Gamma):=\left\{e_{1}, e_{2}, \ldots, e_{n}\right\}$ e a origem e o término de $e_{i}$ é $v$, para todo $1 \leq i \leq n$.

Consideremos agora $K \Gamma$. Note que todas as multiplicações envolvendo caminhos em $\Gamma$ são não nulas por termos um só vértice e que $v=1$. Com isso, $\mathcal{P}$ é um monóide. Definimos então a aplicação

$$
\begin{aligned}
\varphi: & X \longrightarrow \mathcal{P} \\
& x_{i} \mapsto e_{i}
\end{aligned}
$$

Essa aplicação pode ser estendida de maneira única para um homomorfismo de monóides de $X^{*}$ em $\mathcal{P}$. Denotamos essa extensão por $\varphi^{\prime}$. Mas como $\varphi^{\prime}$ leva elementos de uma base de $K\langle X\rangle$ em elementos de uma base de $K \Gamma$, podemos estendê-la de maneira única para uma aplicação linear de $K\langle X\rangle$ em $K \Gamma$. Não é difícil verificar que essa extensão é um homomorfismo de anéis (isso segue, na realidade, do fato de ela ser linear e de sua restrição à base $X^{*}$ ser um homomorfismo de monóides). Finalmente, uma verificação rotineira nos mostra que ela é bijetora, fornecendo um isomorfismo de álgebras entre $K\langle X\rangle$ e $K \Gamma$.

\subsection{Linguagens Formais e Autômatos}

Apresentaremos nesta seção alguns pontos bem conhecidos relacionados à Teoria de Linguagens Formais e Autômatos. Relembramos que os conceitos vistos aqui serão utilizados exclusivamente no Capítulo 5. Ademais, uma vez que esses conceitos são explorados exaustivamente na literatura [HU79, $\operatorname{LSS}^{+} 79$, Beri9], faremos apenas uma apresentação resumida e focada naquilo de que precisaremos nesse capítulo.

Consideramos inicialmente um alfabeto $X$ e o monóide livre sobre $X$. Abordaremos algumas operações envolvendo linguagens sobre $X$. Lembramos que $X^{*}$ é um semigrupo. Assim, dadas duas linguagens $L$ e $L^{\prime}$ sobre $X$, seu produto é definido por: 


$$
L L^{\prime}:=\left\{u v \mid u \in L \text { e } v \in L^{\prime}\right\}
$$

Com esse produto em mãos, dada uma linguagem $L$, podemos definir indutivamente o conjunto $L^{n}$, da seguinte maneira:

$$
L^{0}:=\{1\} \text { e } L^{n}:=L^{n-1} L
$$

Definimos ainda a operação estrela (de Kleene) por:

$$
L^{*}:=\bigcup_{i=0}^{\infty} L^{i}
$$

Em outras palavras, $L^{*}$ é o monóide gerado por $L$. Deve estar claro que o monóide livre sobre $X$ pode ser visto como o resultado da aplicação da operação estrela a $X$. Dessa forma, a notação que temos utilizado para denotar o monóide livre é consistente com a acima apresentada para a operação estrela.

Por conveniência, definimos a operação mais: ${ }^{5}$

$$
L^{+}:=L L^{*}=L^{*} \backslash\{1\}=\bigcup_{i=1}^{\infty} L^{i}
$$

Note mais uma vez que a notação aqui utilizada para a operação mais concorda com a utilizada para denotar o semigrupo livre gerado por $X$.

As operações produto de linguagens, mais, estrela de Kleene e união de linguagens são chamadas de operações racionais. A partir delas, podemos definir o importante conceito de linguagem racional.

Definição 1.5.1 (Linguagem Racional) Uma linguagem $L$ sobre $X$ é dita racional (ou regular) se ela pode ser obtida a partir dos conjuntos unitários $\{w\}$, com $w$ em $X$ e do conjunto vazio através de um número finito de aplicações das operações união, produto e estrela.

Ainda, denotaremos a família de todas as linguagens racionais sobre $X$ por $\operatorname{Rac}(X)$.

É bem conhecido o fato de que linguagens racionais podem ser representadas por expressões racionais. Definimos uma expressão racional indutivamente (no número de operações racionais que ela contém), da seguinte forma:

$\emptyset$ e $w$ são expressões racionais, para todo $w$ em $X$.

Se $L$ é uma linguagem finita, $L$ é uma expressão racional.

Se $e_{1}$ e $e_{2}$ são duas expressões racionais então $\left(e_{1}\right), e_{1} \cup e_{2}, e_{1} e_{2}, e^{+}$e $e_{1}^{*}$ são expressões racionais.

Ilustramos essa discussão com alguns exemplos.

\footnotetext{
${ }^{5}$ É claro que se trata apenas de uma notação e não de uma nova operação. Por outro lado, também deve ficar evidente que a operação estrela pode ser definida em termos da operação mais.
} 
Exemplo 1.5.1 A expressão $e_{1}$ dada por:

$$
e_{1}:=x(y x)^{*} \cup(x y)^{*} y^{+} x^{+}
$$

corresponde à linguagem $L_{1}$ dada por:

$$
L_{1}:=\left\{x(y x)^{n} \in X^{*} \mid n \geq 0\right\} \cup\left\{(x y)^{n} y^{k} x^{l} \in X^{*} \mid n \geq 0, k, l \geq 1\right\}
$$

A expressão $e_{2}$, dada por:

$$
e_{2}:=x\left(y^{4}\right)^{*}(y x)^{*} \cup x\left(y^{2}\right)^{+}
$$

corresponde à linguagem $L_{2}$ dada por:

$$
L_{2}:=\left\{x\left(y^{4}\right)^{n}(y x)^{k} \in X^{*} \mid n, k \geq 0\right\} \cup\left\{x\left(y^{2}\right)^{n} \in X^{*} \mid n \geq 1\right\}
$$

$\Delta$

Dessa forma, uma maneira bastante usual de mostrar que uma dada linguagem $L$ é racional é exibir uma expressão racional para $L$. No restante do trabalho, cometeremos um abuso de linguagem e identificaremos uma expressão racional com a linguagem racional que ela representa.

Seguimos com algumas propriedades importantes das linguagens racionais. As demonstrações para os resultados apresentados aqui podem ser vistos em muitos textos clássicos de Teoria de Linguagens Formais e Autômatos.

Proposição 1.5.1 A família das linguagens racionais sobre um alfabeto $X$ é fechada por: união, produto, operação estrela, operação mais, intersecção, complementação (em $X^{*}$ ) e diferença.

Proposição 1.5.2 Sejam $X$ e $\Sigma$ dois alfabetos e seja

$$
\varphi: X^{*} \longrightarrow \Sigma^{*}
$$

um homomorfismo de monóides. Então, se $L$ é uma linguagem racional sobre $X, \varphi(L)$ é uma linguagem racional sobre $\Sigma$.

Inversamente, se $R$ é uma linguagem racional sobre $\Sigma$, então $\varphi^{-1}(R)$ é uma linguagem racional sobre $X$.

Definição 1.5.2 (Autômato Finito) Um autômato (finito) (ou X-autômato) é uma máquina $\mathcal{A}=(Q, X, I, T, \gamma)$ composta de:

- Um alfabeto $X$.

- Um conjunto finito $Q$ de estados.

- Subconjuntos $I$ e $T$ de $Q$ de estados iniciais e finais, respectivamente.

- Uma função de transição $\gamma: Q \times X \longrightarrow \mathcal{P}(Q)$. 
Podemos estender a função de transição $\gamma$ para uma função de $\mathcal{P}(Q) \times X^{*}$ em $\mathcal{P}(Q)$, da seguinte forma:

Se $q$ é um estado de $A$, então $\gamma(q, 1)=q$.

Se $A$ é um subconjunto de $Q$, definimos $\gamma(A, x)=\cup_{q \in A} \gamma(q, x)$, para todo $x$ em $X$.

Dada uma palavra $u=x_{1} x_{2} \cdots x_{n}\left(\operatorname{com} x_{i}\right.$ em $X$, para cada $\left.1 \leq i \leq n\right)$, definimos $\gamma(q, u)=\gamma\left(\gamma\left(q, x_{1} \cdots x_{n-1}\right), x_{n}\right)$.

Denotaremos essa extensão de $\gamma$ também por $\gamma$.

Um autômato $\mathcal{A}=(Q, X, I, T, \gamma)$ pode ser visto como um grafo $\Gamma$, cujos arcos são rotulados por elementos de $X$. Isso pode ser feito tomando o conjunto de vértices de $\Gamma$ como $Q$ e determinando seu conjunto de arcos pela função de transição $\gamma$, da seguinte maneira. Para cada par de estados $q_{1}$ e $q_{2}$ de forma que $q_{2}=\varphi\left(q_{1}, u\right)$, para algum $u$ em $X$, inserimos um arco com ponta inicial $q_{1}$, ponta final $q_{2}$ e rótulo $u$. Chamaremos cada arco rotulado de transição e denotaremos uma transição com ponta inicial $q_{1}$, ponta final $q_{2}$ e rótulo $u$ pela tripla $\left(q_{1}, u, q_{2}\right)$.

Com isso, podemos representar autômatos graficamente de maneira muito similar à que representamos grafos. De fato, chamamos a atenção do leitor para o fato de que, pela discussão acima, podemos ver autômatos como grafos (com arcos rotulados), de maneira que os conceitos, definições e notações vistos para grafos também se aplicam para autômatos.

Em nossas representações gráficas de autômatos, denotaremos estados finais por círculos cheios e estados não finais por círculos vazios. Ainda, um estado inicial $q$ será denotado com uma flecha dupla incidente a $q$. Apresentamos um exemplo de autômato.

Exemplo 1.5.2 A representação gráfica de um autômato da forma $\mathcal{A}=(Q, X, I, T, \gamma)$ pode ser vista abaixo.

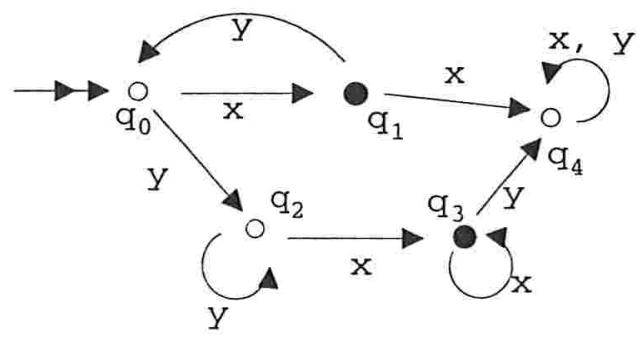

Neste exemplo, $Q=\left\{q_{0}, q_{1}, q_{2}, q_{3}, q_{4}\right\}, X=\{x, y\}, I=\left\{q_{0}\right\}, T=\left\{q_{1}, q_{3}\right\}$ e a função de transição $\gamma$ é dada por:

$$
\begin{array}{ll}
\gamma\left(q_{0}, x\right)=\left\{q_{1}\right\} & \gamma\left(q_{0}, y\right)=\left\{q_{2}\right\} \\
\gamma\left(q_{1}, x\right)=\left\{q_{4}\right\} & \gamma\left(q_{1}, y\right)=\left\{q_{0}\right\} \\
\gamma\left(q_{2}, x\right)=\left\{q_{3}\right\} & \gamma\left(q_{2}, y\right)=\left\{q_{2}\right\} \\
\gamma\left(q_{3}, x\right)=\left\{q_{3}\right\} & \gamma\left(q_{3}, y\right)=\left\{q_{4}\right\} \\
\gamma\left(q_{4}, x\right)=\left\{q_{4}\right\} & \gamma\left(q_{4}, y\right)=\left\{q_{4}\right\}
\end{array}
$$


Ao denotarmos autômatos graficamente, convencionaremos que os símbolos indicados em cada arco representam seus rótulos e não um nome para o arco em si, como fizemos para grafos mais gerais.

Um caminho bem-sucedido em um autômato $\mathcal{A}=(Q, X, I, T, \gamma)$ é um caminho cuja origem pertence a $I$ e cujo término pertence a $T$.

Dado um caminho $c$ em um autômato $\mathcal{A}$, diremos que o rótulo do caminho $c$ é a concatenação dos rótulos dos arcos que compõem $c$. Convencionamos que o rótulo de um caminho de comprimento zero é a palavra vazia. Denotaremos o rótulo de um caminho $c$ por $|c|$. Ainda, se $c$ é um caminho em $\mathcal{A}$, tal que $|c|=u$, diremos que $c$ soletra $u$.

Uma palavra $u$ sobre $X$ é reconhecida pelo autômato $\mathcal{A}=(Q, X, I, T, \gamma)$ se $\gamma(I, u) \cap T \neq \emptyset$. Equivalentemente, $u$ é reconhecida por $\mathcal{A}$ se e somente se existe um caminho bem-sucedido em $\mathcal{A}$ que soletra $u$.

O comportamento do autômato $\mathcal{A}$ (denotado por $|\mathcal{A}|$ ) é então definido como o conjunto das palavras reconhecidas por $\mathcal{A}$. Mais precisamente,

$$
|\mathcal{A}|:=\left\{u \in X^{*} \mid \gamma(I, u) \cap T \neq \emptyset\right\}
$$

Se uma linguagem $L$ é o comportamento de $\mathcal{A}$, diremos também que $L$ é reconhecida por $\mathcal{A}$.

No Exemplo 1.5.2, não é difícil ver que o comportamento de $\mathcal{A}$ é a linguagem $L_{1}$ vista no Exemplo 1.5.1.

Dizemos que dois $X$-autômatos $\mathcal{A}$ e $\mathcal{B}$ são equivalentes se $|\mathcal{A}|=|\mathcal{B}|$.

Comentamos brevemente que existe uma teoria de minimização de autômatos (e ela é descrita em todos os os textos clássicos na área). Um dos principais resultados dessa teoria afirma que, dado um autômato $\mathcal{A}$, existe um algoritmo que devolve um autômato $\mathcal{B}$, equivalente a $\mathcal{A}$, com a seguinte propriedade: se $\mathcal{C}$ é um autômato equivalente a $\mathcal{B}$, então o número de estados de $\mathcal{C}$ não é menor que o número de estados de $\mathcal{B}$.

Um autômato $\mathcal{A}=(Q, X, I, T, \gamma)$ é determinístico se $\operatorname{card}(I)=1$ e $\operatorname{card}(\gamma(q, u))=1$, para todo $\operatorname{par}(q, u)$ de $Q \times X$. Vale notar que o autômato $\mathcal{A}$ definido no Exemplo 1.5.2 é determinístico. Abaixo segue um exemplo de autômato não-determinístico.

Exemplo 1.5.3 Considere o autômato $\mathcal{B}=(Q, X, I, T, \gamma)$ representado abaixo:

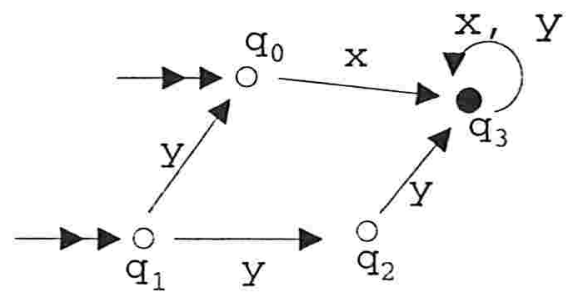

No exemplo, $Q=\left\{q_{0}, q_{1}, q_{2}, q_{3}\right\}, X=\{x, y\}, I=\left\{q_{0}, q_{1}\right\}, T=\left\{q_{3}\right\}$ e $\gamma$ é dada por:

$$
\begin{array}{ll}
\gamma\left(q_{0}, x\right)=\left\{q_{3}\right\} & \gamma\left(q_{0}, y\right)=\emptyset \\
\gamma\left(q_{1}, x\right)=\emptyset & \gamma\left(q_{1}, y\right)=\left\{q_{0}, q_{2}\right\} \\
\gamma\left(q_{2}, x\right)=\emptyset & \gamma\left(q_{2}, y\right)=\left\{q_{3}\right\} \\
\gamma\left(q_{3}, x\right)=\left\{q_{3}\right\} & \gamma\left(q_{3}, y\right)=\left\{q_{3}\right\}
\end{array}
$$


Não é difícil ver que o comportamento de $\mathcal{B}$ é $x X^{*} \cup y x X^{*} \cup y^{2} X^{*}$. $\Delta$

É bem conhecido o fato de que se $\mathcal{A}=(Q, X,\{i\}, T, \gamma)$ é um autômato determinístico, então, para cada par $(q, u)$ em $Q \times X^{*}$, existe um único caminho em $\mathcal{A}$ com origem $q$ e que soletre $u$.

Proposição 1.5.3 Todo autômato é equivalente a um autômato deterministico.

Definição 1.5.3 (Linguagem Reconhecível) Uma linguagem sobre um alfabeto $X$ é reconhecivel se for o comportamento de um $X$-autômato deterministico.

Denotamos a familia de todas as linguagens reconheciveis sobre $X$ por $\operatorname{Rec}(X)$.

O seguinte teorema é certamente um dos mais importantes em Teoria de Linguagens Formais.

Teorema 1.5.4 (Kleene, 1956) Se $X$ é um alfabeto, então

$$
\operatorname{Rac}(X)=\operatorname{Rec}(X)
$$

Algumas vezes será importante encontrar linguagens que não sejam racionais. O seguinte resultado é conhecido como Lema da Iteração (Iteration Lemma ou Pumping Lemma, no original) e é muito útil para esse propósito.

Proposição 1.5.5 (Lema da Iteração) Seja L uma linguagem racional sobre $X$. Então existe um inteiro $n$, de modo que, para toda palavra $w$ em $L$ de comprimento maior ou igual a $n$, $w$ pode ser escrita como $w=u v z$, de modo que $\|u v\| \leq n$, $\|v\| \geq 1$ e, para todo $i \geq 0$, uv $v^{i}$ pertence a $L$.

Exemplo 1.5.4 Considere a linguagem $L$ dada por $L=\left\{x^{k} y^{k} \mid k \geq 1\right\}$. Vamos mostrar que $L$ não é uma linguagem racional, fazendo uso do Lema da Iteração.

Com efeito, suponha por absurdo que $L$ é racional. Então, pelo Lema da Iteração, existe uma constante $n$ com as propriedades descritas no lema. Tomamos então uma palavra $w=x^{k} y^{k}$, com $k>n$. Claro que $w$ pertence a $L$.

Por outro lado, pelo Lema da Iteração, podemos fatorar $w$ como $w=u v z$, com $\|u v\| \leq n$. Mas então, $u=x^{j}, v=x^{h}$, para alguns $0 \leq j<n$ e $1 \leq h \leq n$ e $z=x^{l} y^{k}$, para algum $1 \leq l<k$. Note que $j+h+l=k$.

Logo, o Lema da Iteração implica que $u v^{2} z=x^{j+2 h+l} y^{k}$ pertence a $L$, nos levando a uma contradição com a definição de $L$, uma vez que $j+2 h+l>k$. Assim, concluímos que $L$ não é racional.

De forma bastante similar, pode-se utilizar o Lema da Iteração para mostrar que a linguagem $L^{\prime}:=\left\{u \in X^{*} \mid\|u\|_{x}=\|u\|_{y}\right\}$ não é racional.

\subsection{Algoritmos e Computabilidade}

Apresentamos nesta seção alguns conceitos e notações envolvendo algoritmos e computabilidade que serão utilizados ao longo de todo o texto.

Lembramos que, informalmente, um procedimento (efetivo) é uma seqüência de passos que podem ser executados por um "agente computacional". Supõe-se também que todo procedimento 
possua uma descrição finita. Note que cada passo de um procedimento dever ser efetivo; em particular, cada passo deve ser não ambíguo e executado em tempo finito. Um procedimento recebe dados como entrada e produz uma saída.

Usualmente, ao descrever um procedimento $\mathrm{A}$, indicaremos o conjunto de valores $V$ que são aceitos como entrada por A. Diremos então que um elemento de $V$ é uma entrada válida para A.

Um algoritmo é um procedimento cuja execução termina para qualquer entrada válida fornecida. Essa distinção entre algoritmo e procedimento será largamente utilizada ao longo de todo o trabalho, uma vez que encontraremos procedimentos (e entradas válidas para eles) que não terminam sua computação.

Dado um procedimento $\mathrm{A}$, que recebe como entrada $n$ parâmetros, utilizaremos as notações $s=\mathrm{A}\left(v_{1}, v_{2}, \ldots v_{n}\right)$ ou $s:=\mathrm{A}\left(v_{1}, v_{2}, \ldots v_{n}\right)$ para indicar que $s$ é o valor da saída do procedimento $\mathrm{A}$ ao receber como entrada os valores $v_{1}, v_{2} \ldots v_{n}$.

Ao descrever algoritmos e procedimentos, utilizaremos a notação definida por Cormen, Leiserson, Rivest e Stein [CLRS01]. De acordo com essa notação, cada procedimento começa com um nome, seguido de uma lista de parâmetros e devolve algum valor. Assumiremos também que algoritmos podem modificar os valores dos parâmetros recebidos como entrada e que podem fazer uso de um número infinito de variáveis, indexadas por algum conjunto (um exemplo desse uso pode ser visto no Algoritmo 4.3.1). ${ }^{6}$

Uma atribuição será denotada por

$$
v \leftarrow a
$$

indicando que a variável $v$ recebe o valor $a$. Note que variáveis poderão armazenar objetos de quaisquer estruturas algébricas que abordarmos, bem como conjuntos.

As estruturas de controle que utilizaremos ao longo do trabalho são:

- devolva (expressão)

- se (condição) então ... senão ...

- enquanto (condição) faça ...

- para cada (elemento em conjunto) faça ...

- repita ... até (condição)

O leitor não familiarizado com essa notação deve notar que a estrutura repita executa seu corpo de instruções enquanto a condição for falsa. Ainda, o corpo de uma instrução repita é executado ao menos uma vez (a condição é testada ao final de cada iteração e não no início).

Uma chamada de função será denotada pelo nome da função, seguida da lista de valores passados como parâmetro para ela.

Faremos uso também de alguns outros tipos de operações, que embora não sejam elementares, serão tratadas com tal neste trabalho. São elas:

\footnotetext{
${ }^{6}$ Conforme ficará evidente nas descrições de procedimentos adiante, utilizaremos um número infinito de variáveis apenas com o propósito de tornar algumas dessas descriçōes mais adequadas a certas demonstraçōes. Entretanto, deve ficar claro que, sempre que utilizarmos esse recurso, uma modificação simples na descrição do procedimento permitirá que ele faça uso de quantidade finita de armazenamento, sem prejuízo para seu funcionamento.
} 
- As seguintes operações envolvendo conjuntos (finitos): união, intersecção, diferença e complementação.

- A partir de um elemento $f$ e um conjunto finito $C$, decidir se $f$ pertence a $C$.

- A partir de um conjunto finito $C$ e uma condição $Q$ (que envolva apenas manipulações algébricas "simples"), decidir se existe um elemento em $C$ que satisfaz $Q$ e devolvê-lo.

- Computação das operações algébricas sobre corpos, álgebras e demais estruturas algébricas.

Vale ressaltar que, dependendo do contexto, algumas das operações acima descritas podem não ser efetivas. Contudo, as assumiremos como elementares nas descrições dos algoritmos e comentários sobre sua efetividade serão feitos oportunamente, quando forem pertinentes.

Ainda, em demonstrações e demais discussões com referência a um procedimento $\mathrm{A}$, faremos um abuso de linguagem e identificaremos uma variável $f$ utilizada em A com seu valor (desde que não haja possibilidade de confusão).

Algumas descrições de procedimentos que apresentamos neste trabalho são não determinísticas, ou seja, permitem uma série de escolhas alternativas para uma mesma entrada durante uma execução do procedimento (e eventualmente fornecem saídas diferentes para diferentes escolhas). Como é usual, assumiremos que qualquer escolha pode ser feita e que qualquer uma é igualmente boa para as propriedades que nos interessam.

Dado um problema $P$, diremos que $P$ é computável, se existe um algoritmo A que resolve $P$. Um problema é dito não computável se ele não for computável. Em outras palavras, $P$ é não computável se não existe nenhum algoritmo que resolve $P$.

Ainda, chamaremos um problema de decisão computável de decidível e um problema de decisão não computável de indecidível. 



\section{MotivaÇÃo e BASES De Gröbner COMUtativas}

Este capítulo apresenta os principais conceitos da Teoria de Bases de Gröbner. Iniciamos com um pequeno histórico da área e alguns problemas que motivaram seu estudo, seguindo com uma apresentação bastante sucinta da teoria para anéis de polinômios comutativos.

\subsection{Histórico e Motivação}

As Bases de Gröbner para ideais do anel de polinômios comutativo, $K\left[x_{1}, \ldots, x_{n}\right]$, foram introduzidas por Buchberger em sua tese de doutorado em 1965 [Buc65] e desde então desempenham um papel muito importante na área de Álgebra Computacional.

Grande parte dessa importância se deve ao fato de, nesse contexto, elas fornecerem uma solução algorítmica para o problema da pertinência para ideais e, conseqüentemente, para o problema da igualdade em quocientes do anel de polinômios comutativo. Descrições mais precisas desses dois problemas são apresentadas em seguida.

Problema da pertinência para ideais de polinômios Dado $f \in K\left[x_{1}, \ldots, x_{n}\right]$ e um conjunto gerador para um ideal $I$ de $K\left[x_{1}, \ldots, x_{n}\right]$, decida se $f$ pertence a $I$.

Problema da igualdade em quocientes do anel de polinômios Dados $f, g$ em $K\left[x_{1}, \ldots, x_{n}\right]$ e um conjunto gerador para um ideal $I$ de $K\left[x_{1}, \ldots, x_{n}\right]$, decida se $f+I=g+I$.

Como dois elementos $f$ e $g$ de $K\left[x_{1}, \ldots, x_{n}\right]$ estão na mesma classe de equivalência do quociente $K\left[x_{1}, \ldots, x_{n}\right] / I$ se e somente se $f-g$ pertence a $I$, fica claro que ao solucionar o problema da pertinência, as Bases de Gröbner fornecem uma solução para o problema da igualdade. Um ponto forte da teoria é a existência dos seguintes algoritmos.

Algoritmo da Divisão Este algoritmo decide a pertinência de um elemento em um ideal, dados o elemento e uma Base de Gröbner para o ideal em questão. Por hora, pode-se pensar em uma Base de Gröbner como um conjunto gerador "especial" do ideal.

Algoritmo de Buchberger Constrói uma Base de Gröbner para um ideal, a partir de um conjunto finito de geradores.

Muitas das idéias envolvidas com a teoria foram estudadas independentemente por Hironaka [Hir64] e Knuth e Bendix [KB70]. O termo "standard base" é bastante presente na literatura e se refere, tipicamente, ao trabalho de Hironaka. 
O termo "Base de Gröbner" foi introduzido por Buchberger posteriormente a sua defesa de tese, em uma homenagem a seu orientador, W. Gröbner. As Bases de Gröbner tiveram como motivação problemas em Geometria Algébrica. Mais especificamente, W. Gröbner levantou a questão de existência de um algoritmo que computasse uma base do quociente $K\left[x_{1}, \ldots, x_{n}\right] / I$, dado um conjunto gerador (finito) para o ideal $I$. Essa base deveria ter a propriedade de permitir que cálculos em $K\left[x_{1}, \ldots, x_{n}\right] / I$ pudessem ser feitos algoritmicamente.

As Bases de Gröbner, em certo sentido, trouxeram um novo impulso para a área de Álgebra Computacional e são abordadas (ao menos o caso comutativo) em muitos textos em nível de pósgraduação e graduação [CLO97, BWV93, AL94, vzGG99]. Ademais, muitos sistemas de computação algébrica incluem pacotes para lidar com Bases de Gröbner (comutativas). Entre eles, podemos citar: Axiom, Maple, Mathematica, CoCoA e Reduce.

As principais idéias envolvidas na generalização da Teoria de Bases de Gröbner para o caso não comutativo foram inicialmente estudada por Bergman [Ber78] e por Bokut [Bok76], mas o primeiro a tratar esta generalização de um ponto de vista mais construtivo foi Mora [Mor86, Mor94]. Mora introduziu um procedimento equivalente ao algoritmo de Buchberger para o caso não comutativo (comumente chamado de procedimento de Mora). Adiaremos maiores discussões sobre a importância dessa generalização para o Capítulo 3 .

Neste capítulo apresentamos de forma bastante sucinta os principais conceitos envolvidos em Teoria de Bases de Gröbner comutativas. Estas mesmas idéias se repetem durante o estudo do caso não comutativo, visto nos Capítulos 3 e 4 . Assim, a leitura desta motivação é bastante indicada para o leitor não familiarizado com a teoria.

Aqui apresentamos também uma breve motivação envolvendo o problema da pertinência. Consideraremos o estudo de tal problema em dois contextos mais simples que $K\left[x_{1}, \ldots, x_{n}\right]$ : em espaços vetoriais de dimensão finita e em $K[x]$.

Vale ressaltar que, dado o caráter introdutório deste capítulo, não nos preocuparemos com detalhes de alguns resultados ou algoritmos apresentados. Tal refinamento será feito ao introduzirmos os análogos das idéias desenvolvidas aqui para o caso não comutativo.

\subsection{O Problema da Pertinência em Subespaços Vetoriais}

Nesta seção consideramos o problema da pertinência para subespaços de um espaço vetorial de dimensão finita. A apresentação deste problema tem o intuito de destacar, em um contexto bastante simples e familiar, alguns conceitos importantes em Teoria de Bases de Gröbner.

Considere um corpo $K$, seja $V=K^{n}$ e fixe uma base ordenada canônica de $V$. Seja ainda $v$ um vetor de $V, W$ um subespaço de $V$ e $B=\left\{b_{1}, b_{2}, \ldots, b_{m}\right\}$ uma base ordenada de $W$. Denotamos por $\left(b_{i}^{1}, b_{i}^{2}, \ldots, b_{i}^{n}\right)$ as coordenadas de cada elemento $b_{i}$ de $B$ com relação à base ordenada canônica de $V$. Consideramos então o problema de decidir se $v$ pertence a $W$.

A primeira e mais simples abordagem para solucionar este problema consiste em resolver um sistema linear. De fato, $v$ pertence a $W$ se e somente se $v$ pode ser escrito como combinação linear de elementos de $B$. Com isso, se considerarmos $v=\left(v^{1}, v^{2}, \ldots, v^{n}\right)$ as coordenadas de $v$ com relação à base canônica de $V$, resta-nos resolver o seguinte sistema linear: 


$$
\left\{\begin{array}{l}
x_{1} b_{1}^{1}+x_{2} b_{2}^{1}+\cdots+x_{m} b_{m}^{1}=v^{1} \\
x_{1} b_{1}^{2}+x_{2} b_{2}^{2}+\cdots+x_{m} b_{m}^{2}=v^{2} \\
\cdots \\
x_{1} b_{1}^{n}+x_{2} b_{2}^{n}+\cdots+x_{m} b_{m}^{n}=v^{n}
\end{array}\right.
$$

Caso o sistema possua solução, teremos que $v$ pertence a $W$ e $v$ não pertence a $W$ caso contrário. ${ }^{1}$

Uma segunda abordagem bastante simples e comum é construir uma matriz $M$ de forma que cada linha de $M$ contenha as coordenadas de um elemento de $B$. Adiciona-se ainda uma linha a $M$ com as coordenadas de $v$ e aplica-se o algoritmo de Gauss-Jordan a $M$. Com isso, temos que $v$ pertence a $W$ se e somente se a matriz resultante de aplicação de Gauss-Jordan a $M$ contiver uma linha nula (i.e. somente com zeros).

Incluímos no Algoritmo 2.2.1 uma descrição informal do algoritmo de Gauss-Jordan para conveniência do leitor. Contudo, omitimos detalhes e maiores comentários sobre esse algoritmo, pois sua apresentação neste texto tem um caráter apenas ilustrativo de conceitos que serão importantes adiante. O leitor interessado encontra maiores detalhes em muitos textos clássicos; a versão aqui apresentada é uma versão simplificada de uma abordagem mais algorítmica [Feo99].

Antes de proceder com a descrição do algoritmo, fixamos alguma notação que facilitará a leitura do que segue: se $M$ é uma matriz, denotamos por $M[i$,$] a i$-ésima linha de $M$ e por $M[, j]$ sua $j$-ésima coluna.

\section{Algoritmo 2.2.1 - Algoritmo de Gauss-Jordan (GAUSSJORDAN) \\ O algoritmo recebe como entrada uma matriz $M p \times q$ e devolve uma matriz $M^{\prime}$ com as mesmas dimensões de $M$. O algoritmo faz uso ainda de um conjunto de índices $P$. Inicialmente, $M^{\prime}=M \mathrm{e}$ $P$ é vazio. A cada iteração, o algoritmo considera uma coluna $j$ de $M$, da esquerda para a direita e cada iteração consiste no seguinte:}

Se todas as colunas de $M$ já foram consideradas, o algoritmo pára e devolve $M$.

Caso contrário, seja $j$ a primeira coluna de $M$ (da esquerda para a direita) ainda não considerada em nenhuma iteração. Se não existe uma linha $i$, com $i \notin P$ tal que $M[i, j] \neq 0$, o algoritmo passa para a próxima iteração. Caso contrário, seja $i$ uma linha de $M$ com as propriedades acima (essa linha é comumente chamada de pivô da iteração). Constrói-se, então, a matriz $M^{\prime} p \times q$ definida da seguinte maneira:

$$
\begin{aligned}
& M^{\prime}[i,]=\frac{1}{M[i, j]} M[i,] \\
& M^{\prime}[h,]=M[h,]-\frac{M[h . j]}{M[i . j]} M[i,], \text { para cada } h \neq i
\end{aligned}
$$

Ainda, neste caso, o algoritmo faz $P^{\prime}=P \cup\{i\}$. Após isso, inicia-se uma nova iteração com $M^{\prime}$ no lugar de $M$ e $P^{\prime}$ no lugar de $P$.

Ilustramos essa segunda abordagem com um exemplo.

\footnotetext{
${ }^{1}$ Vale mencionar o fato de que para esta abordagem não é necessário que $B$ seja uma base; basta ser um conjunto gerador.
} 
Exemplo 2.2.1 Tome $V=\mathbb{Q}^{6}, W$ o subespaço gerado pela base

$$
B=\{(1,0,-1,3,3,2),(0,-1,2,4,3,2),(2,3,0,2,5,-2)\}
$$

e $v=(1,-1,2,8,7,4)$. O algoritmo de Gauss-Jordan executará três iterações, correspondentes às três primeiras colunas da matriz. Apresentamos a matriz $M^{\prime}$ calculada pelo algoritmo ao final de cada iteração, assumindo que ele escolheu como pivô as linhas 1, 2 e 3 da matriz, respectivamente para cada iteração.

$$
\begin{aligned}
& {\left[\begin{array}{cccccc}
1 & 0 & -1 & 3 & 3 & 2 \\
0 & -1 & 2 & 4 & 3 & 2 \\
2 & 3 & 0 & 2 & 5 & -2 \\
1 & -1 & 2 & 8 & 7 & 4
\end{array}\right] \simeq\left[\begin{array}{cccccc}
1 & 0 & -1 & 3 & 3 & 2 \\
0 & -1 & 2 & 4 & 3 & 2 \\
0 & 3 & 2 & -4 & -1 & -6 \\
0 & -1 & 3 & 5 & 4 & 2
\end{array}\right] \simeq} \\
& {\left[\begin{array}{cccccc}
1 & 0 & -1 & 3 & 3 & 2 \\
0 & 1 & -2 & -4 & -3 & -2 \\
0 & 0 & 8 & 8 & 8 & 0 \\
0 & 0 & 1 & 1 & 1 & 0
\end{array}\right] \simeq\left[\begin{array}{cccccc}
1 & 0 & 0 & 4 & 4 & 2 \\
0 & 1 & 0 & -2 & -1 & -2 \\
0 & 0 & 1 & 1 & 1 & 0 \\
0 & 0 & 0 & 0 & 0 & 0
\end{array}\right]}
\end{aligned}
$$

Com isso, podemos concluir que $v$ pertence a $W$. ^

Por fim, apresentamos uma terceira abordagem para o problema da pertinência em subespaços. Embora ela seja menos usual e direta que as anteriores, ela desperta a atenção para uma série de elementos que aparecem, em especial, na segunda abordagem e que serão de grande valia para a generalização desse problema para os anéis de polinômios comutativo e não comutativo. ${ }^{2}$

Em linhas gerais, o problema da pertinência pode ser resolvido em duas etapas: primeiro encontramos uma base "especial" $G$ de $W$, que chamaremos de Base de Gauss (a definição é dada adiante). De posse dessa base, utilizamos a Redução Incompleta de Gauss para "dividir" o vetor $v$ pelos elementos de $G$.

Iniciamos com o análogo de um conceito central em Teoria de Bases de Gröbner para espaços vetoriais.

Definição 2.2.1 (Coordenada Líder) Chamamos de coordenada líder de um vetor não nulo $v$ (denotada por $\widehat{v}$ ) a menor coordenada não nula de $v$.

A fim de exemplificar esse conceito, podemos tomar os vetores $v_{1}:=(3,0,2)$ e $v_{2}:=(0,0,1)$ de $\mathbb{Q}^{3}$. Neste caso, $\widehat{v_{1}}=1$ e $\widehat{v_{2}}=3$.

Definição 2.2.2 (Base de Gauss) Uma base $G$ de um espaço vetorial $V$ é dita uma Base de Gauss se ela satisfaz as seguintes propriedades:

- Não existem dois vetores em $G$ com a mesma coordenada lider.

- Se um vetor não nulo u pertence a $V$, então existe um elemento $g$ em $G$ tal que $\widehat{u}=\widehat{g}$.

\footnotetext{
${ }^{2}$ Talvez haja um certo exagero em chamar o que segue de terceira abordagem. Mais justo seria dizer que será feito um "refinamento" da segunda abordagem
} 
Para obter uma Base de Gauss $G$ a partir de uma base qualquer $B$ de $W$, basta aplicar o algoritmo de Gauss-Jordan, como mostra o seguinte resultado.

Lema 2.2.1 Seja $A=\left\{a_{1}, a_{2}, \ldots, a_{k}\right\}$ um conjunto gerador de um espaço vetorial $W$. Então, se fornecermos a matriz $M$ dada por:

$$
M=\left[\begin{array}{c}
a_{1} \\
a_{2} \\
\vdots \\
a_{k}
\end{array}\right]
$$

para o algoritmo de Gauss-Jordan, a matriz $M^{\prime}$ será da forma

$$
M^{\prime}=\left[\begin{array}{c}
g_{1} \\
g_{2} \\
\vdots \\
g_{k}
\end{array}\right]
$$

Além disso, existe $m \leq k$ tal que $g_{m+1}=g_{m+2}=\cdots=g_{k}=0$ e $G:=\left\{g_{1}, g_{2}, \ldots, g_{m}\right\}$ é uma Base de Gauss para W.

Demonstração. Utilizando propriedades bem conhecidas do algoritmo de GaussJordan é fácil ver que $M^{\prime}$ será da forma descrita e que os vetores $g_{1}, g_{2}, \ldots, g_{m}$ formam uma base de $W$. Resta, portanto, mostrar que a base $G$ é de Gauss.

Vejamos primeiramente que não existem dois elementos de $G$ com a mesma coordenada líder. De fato, suponha que existem linhas $h_{1}$ e $h_{2}$ de $M^{\prime}$ (representando elementos $g_{i_{1}}$ e $g_{i_{2}}$, respectivamente) tais que $\widehat{g_{i_{1}}}=\widehat{g_{i_{2}}}=j$. Note que $M\left[h_{1}, k\right]=M\left[h_{2}, k\right]=0$, para todo $k<j$. Com isso, no início da $j$-ésima iteração, nenhuma dessas linhas pertence a $P$ e alguma linha será escolhida para ser o pivô dessa iteração; seja $h_{1}$ essa linha (podemos supor isso sem perda de generalidade). Então, ao final dessa iteração, $M^{\prime}\left[h_{2}, j\right]$ será igual a 0 e $M^{\prime}\left[h_{1}, j\right]$ será igual a 1 , donde $\widehat{g_{i_{2}}} \neq \widehat{g_{i_{1}}}=j$, uma contradição.

Para ver que a coordenada líder de todo elemento não nulo de $W$ é igual a coordenada líder de algum elemento de $G$, tome $u$ vetor não nulo de $W$. Então, $u=\lambda_{1} g_{1}+\lambda_{2} g_{2}+$ $\cdots+\lambda_{m} g_{m}$. Considere $g_{j}$ o elemento com menor coordenada líder dentre os $g_{i}$ com $\lambda_{i} \neq 0$ (ele é único pelo que foi mostrado no parágrafo anterior). Então, pela minimalidade de $g_{i}$, segue que sua coordenada não pode ser cancelada na soma acima e $\widehat{u}=\widehat{g}_{i}$, o que encerra a demonstração.

Uma vez que temos uma Base de Gauss de $W$ em mãos, precisamos "dividir" o vetor $v$ por essa base e verificar o resultado dessa divisão. O processo que chamamos informalmente aqui de divisão é, na realidade, a Redução Incompleta de Gauss, que descrevemos no Algoritmo 2.2.2.

Utilizamos a notação $c l(u)$ para denotar o valor da coordenada líder de um vetor $u$. Ainda, se, para um dado vetor $v$ e uma dada base $G, h:=\operatorname{RedinCGaUss}(G, v)$, diremos que o vetor $v$ se reduz para $h$ sobre $G$. Note que o coeficiente líder de $h$ aumenta a cada iteração. Isso nos garante que a Redução Incompleta de Gauss pára ( $\widehat{h}$ não pode ser maior que a dimensão de $V$ ). O próximo resultado nos mostra como utilizar esse algoritmo para o problema da pertinência. 


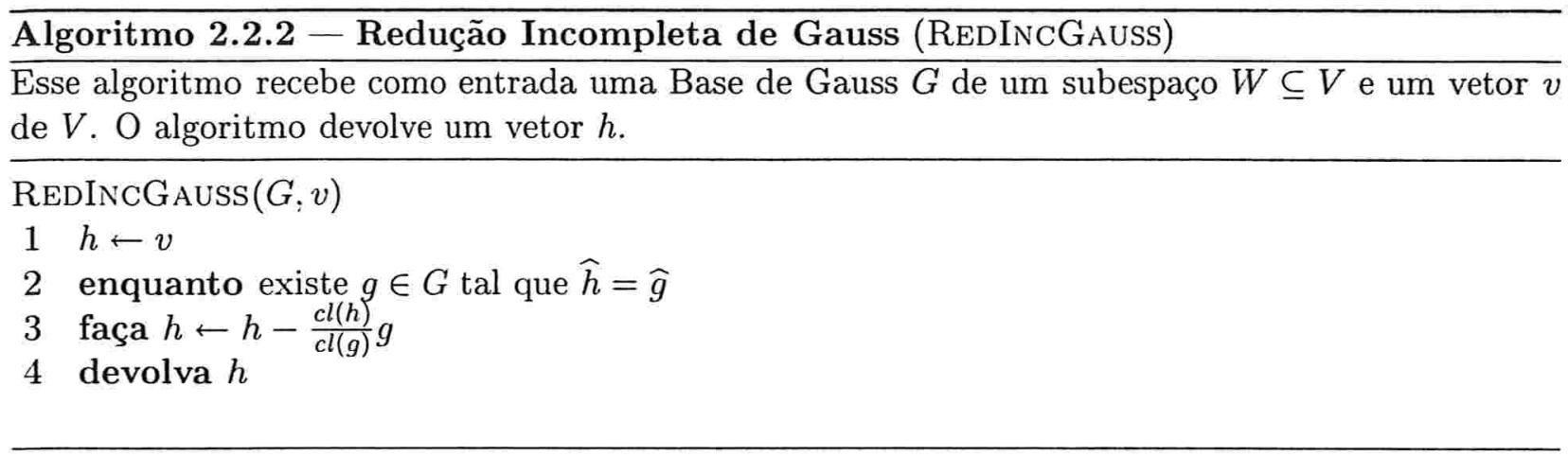

Lema 2.2.2 Seja $G$ uma Base de Gauss de um subespaço $W$ de $V$ e $v \in V$. Então $v \in W$ se e somente se $h:=\operatorname{REDINCGauss}(G, v)$ for igual a 0 .

Demonstração. Se $v=0$, então $h$ será igual a 0 e não há o que mostrar. Supomos então no que segue que $v \neq 0$.

$(\Leftarrow)$

Se $h=0$, então existe uma seqüência de $g_{i}$ 's tal que

$$
v-\lambda_{1} g_{i_{1}}-\lambda_{2} g_{i_{2}}-\cdots-\lambda_{k} g_{i_{k}}=0
$$

donde $v$ se escreve como combinação linear de elementos da base $G$ de $W$.

$(\Rightarrow)$

Essa parte da prova segue por contradição. Suponha que essa implicação do lema é falsa. Então existem elementos de $W$ para os quais a saída de $\operatorname{RedincGauss}\left(G, v_{i}\right)$ é diferente de zero. Dentre tais elementos que contradizem a implicação, escolhemos um elemento $v$ que possua coordenada líder maximal; ou seja, qualquer outro elemento $u$ de $W$ com coordenada líder maior que a coordenada líder de $v$ (se existir) satisfaz $\operatorname{RedincGauss}(G, u)=0$.

Mas, como $v \in W$, segue que $v$ se escreve como combinação linear dos elementos de $G$. Como $G$ é Base de Gauss, podemos eliminar os termos dessa soma com coeficiente nulo e reordená-los de forma que $v=\lambda_{1} g_{i_{1}}+\lambda_{2} g_{i_{2}}+\cdots+\lambda_{k} g_{i_{k}}$, satisfazendo $\widehat{g_{i_{1}}}<\widehat{g_{i_{2}}}<\cdots<\widehat{g_{i_{k}}}$ e $\widehat{v}=\widehat{g_{i_{1}}}$. Com isso, quando $v$ é fornecido para o algoritmo de Redução Incompleta de Gauss, na primeira iteração, o algoritmo seleciona o único elemento $g \in G$ que satisfaz $\widehat{g}=\widehat{v}: g_{i_{1}}$ (a existência e unicidade de tal elemento são dadas pelas duas propriedades de Bases de Gauss destacadas na Definição 2.2.2).

Então $h \leftarrow v-\lambda_{1} g_{i_{1}}$; note que $h \in W$ e $\widehat{h}<\widehat{v}$ ou $h=0$. Se $h=0$ não há mais o que mostrar; senão, pela maximalidade de $\widehat{v}$, segue que $h$ se reduz para zero sobre $G$, donde $\operatorname{RedINCGauss}(G, v)=0$.

Ilustramos esses conceitos retomando o Exemplo 2.2.1.

Exemplo 2.2.2 Tome $V=\mathbb{Q}^{6}, W$ o subespaço gerado pela base 


$$
B=\{(1,0,-1,3,3,2),(0,-1,2,4,3,2),(2,3,0,2,5,-2)\}
$$

e $v=(1,-1,2,8,7,4)$.

Se tomamos como pivô das iterações 1,2 e 3 as linhas 1,2 e 3 da matriz respectivamente, a Base de Gauss calculada pelo algoritmo de Gauss-Jordan é $G:=\left\{g_{1}:=\right.$ $\left.(1,0,0,4,4,2), g_{2}:=(0,1,0,-2,-1,-2), g_{3}:=(0,0,1,1,1,0)\right\}$ (os detalhes do funcionamento do algoritmo são os mesmos dos mostrados no exemplo anterior).

Ao aplicarmos a Redução Incompleta de Gauss, obteremos $h=0$, como é de se esperar. $O$ valor de $h$ calculado a cada iteração é dado em seguida:

Primeira iteração: o algoritmo escolhe $g_{1}: h=(0,-1,2,4,3,2)$

Segunda iteração: o algoritmo escolhe $g_{2}: h=(0,0,2,2,2,0)$

Terceira iteração: o algoritmo escolhe $g_{3}: h=(0,0,0,0,0,0)$ e o algoritmo pára.

Encerramos esta seção com um exemplo que mostra a importância da base utilizada na Redução Incompleta de Gauss ser uma Base de Gauss. Considere a base $B=\{(1,2,1),(1,0,2),(0,0,1)\}$ de $\mathbb{Q}^{3}$ e o vetor $v=(1,3,1)$. Naturalmente, $v \in \mathbb{Q}^{3}$. Porém, se aplicamos a redução de Gauss com o conjunto $B$ e escolhemos $(1,2,1)$ na primeira iteração, o algoritmo pára, devolvendo $(0,1,0)$. Mais que isso, se na primeira iteração do algoritmo o vetor $(1,0,2)$ for escolhido, sua saída será $(0,3,-1)$.

Logo, podemos ver que a ordem dos elementos da base escolhidos altera a saída da redução (além da redução não responder corretamente à pergunta de pertinência). Destacamos que quando a base é de Gauss, uma única ordem de escolha de elementos da base é possível pelo fato de não existirem dois elementos com o mesmo coeficiente líder e o algoritmo sempre responde corretamente à pergunta de pertinência.

\subsection{O Problema da Pertinência em $K[x]$}

Consideramos agora o problema da pertinência em $K[x]$. Veremos que muitas das idéias desenvolvidas para espaços vetoriais podem ser aplicadas neste contexto. Ainda, destacaremos alguns elementos que serão fundamentais na apresentação da Teoria de Bases de Gröbner comutativas, que aborda o caso mais geral do anel de polinômios comutativo em várias variáveis: $K\left[x_{1}, \ldots, x_{n}\right]$.

Vale notar que no caso particular em que $n=1$, o algoritmo de Buchberger se reduz exatamente ao Algoritmo de Euclides. O mesmo ocorre com o Algoritmo da Divisão, de modo que o conteúdo desta seção pode ser visto como a particularização da Teoria de Bases de Gröbner comutativas para o caso em que $n=1$.

Seja $I$ um ideal de $K[x]$ e tome $p$ um polinòmio qualquer. Para decidir se $p$ é um elemento de $I$, utilizamos o fato de que em $K[x]$ todo ideal é principal, ou seja $I=\langle h\rangle$, para algum polinômio $h$. Uma vez que temos $h$, basta verificarmos se o resto da divisão de $p$ por $h$ é zero utilizando o Algoritmo da Divisão em $K[x]$.

Com isso, para resolvermos o problema da pertinência de um polinômio $p$ em um ideal $I$, dado um conjunto gerador $\left\{f_{1}, f_{2}, \ldots, f_{n}\right\}$ de $I$, precisamos primeiro encontrar um polinômio $h$ tal que 
$I=\langle h\rangle$ e então aplicar o Algoritmo da Divisão. Para encontrar $h$, fazemos uso do famoso Algoritmo de Euclides, que encontra um máximo divisor comum de dois polinômios. ${ }^{3}$

Iniciamos, então, com o Algoritmo da Divisão (Algoritmo 2.3.1). Utilizamos as notações $\partial(p)$ para denotar o grau do polinômio $p, \widehat{p}$ para denotar seu monômio de maior grau e $c l(p)$ para denotar seu coeficiente dominante.

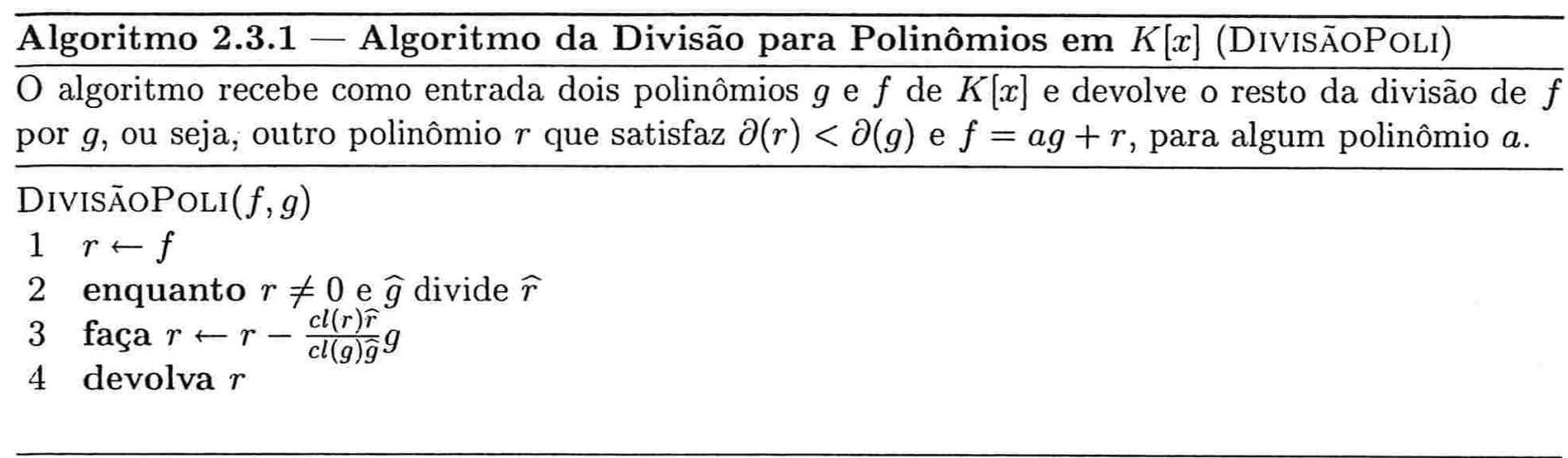

Comentamos que, em geral, o algoritmo também calcula o quociente da divisão (o polinômio $a$ ). Contudo, o omitimos de sua saída por não ser necessário para nossos propósitos.

Uma observação bastante importante com relação a esse algoritmo é o fato dele considerar os termos dos polinômios "em ordem". De fato, podemos imaginar o funcionamento do algoritmo como primeiro ordenando os termos de $g$ e de $f$ de acordo com seu grau, depois considerando os termos de $f$ do maior para o menor e sempre dividindo o termo de $f$ escolhido pelo maior termo de $g .{ }^{4}$

Com efeito, o sucesso do algoritmo depende dessa ordenação (o leitor é convidado a tentar dividir, por exemplo, $f:=x^{5}-x^{2}$ por $g:=x^{2}-x$, considerando $x$ como $\widehat{g}$ no Algoritmo da Divisão) e ela pode ser utilizada para mostrar que o algoritmo pára, uma vez que o grau de $r$ diminui a cada iteração.

Vale ressaltar que nas duas estratégias que utilizamos até agora para resolver o problema da pertinência (tanto para espaços vetoriais quanto para $K[x]$ ) uma ordem nos termos é necessária, bem como é necessário definir, para cada elemento não nulo, seu "termo líder". No caso de espaços vetoriais, a própria noção de coordenada nos deu uma tal ordem e agora podemos utilizar o grau de cada termo para ordená-los totalmente.

Note que em ambos os casos, essas ordens nos fornecem a garantia de que os processos de "divisão" que utilizamos de fato páram, seja com o Algoritmo da Divisão em $K[x]$ ou com a Redução Incompleta de Gauss.

Ao apresentarmos os elementos da Teoria de Bases de Gröbner na Seção 2.4 e no Capítulo 3, generalizações dessa ordenação nos termos e dessa noção de "termo líder" serão apresentadas com um propósito muito parecido ao visto nesses dois exemplos.

Retomando o problema da pertinência em $K[x]$, uma vez que temos o Algoritmo da Divisão em mãos, só nos resta encontrar o polinômio $h$ tal que $I=\langle h\rangle$, a partir de geradores $f_{1}, f_{2}, \ldots, f_{n}$ do ideal I. Para tanto, apresentamos o Algoritmo de Euclides (Algoritmo 2.3.2).

\footnotetext{
${ }^{3}$ Lembramos que o máximo divisor comum de dois polinômios é único a menos de multiplicação por uma constante não nula. Cometeremos então um abuso de notação escrevendo $m=\operatorname{mdc}(f, g)$ para denotar que $m$ é um máximo divisor comum de $f$ e $g$.

${ }^{4}$ Deve estar claro que todo polinômio em $K[x]$ se escreve de forma única como soma de seus termos agrupados e ordenados.
} 


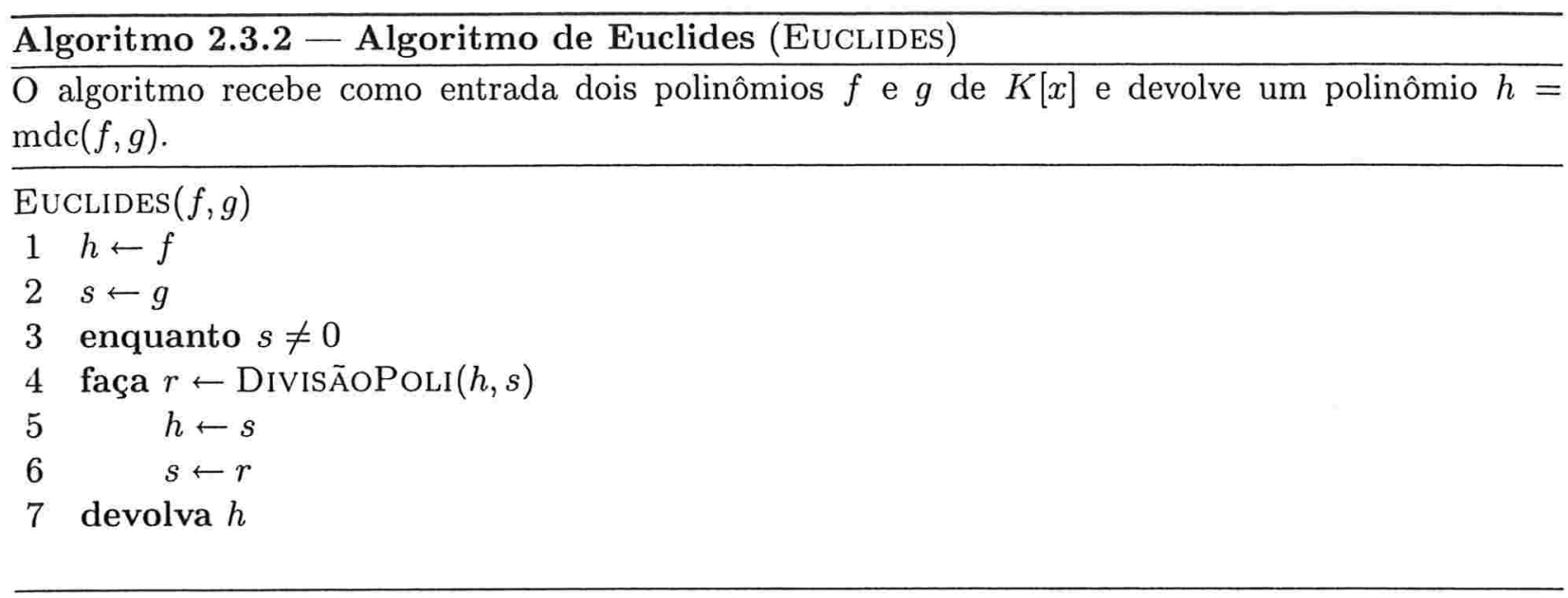

O algoritmo é bem conhecido, de forma que omitiremos maiores comentários sobre seu funcionamento (para uma exposição introdutória, referimo-nos a [CLO97]). Também é bem conhecido o fato de que o polinômio $h$ procurado é um máximo divisor comum de $f_{1}, f_{2}, \ldots, f_{n}$. Além disso, $\operatorname{mdc}\left(f_{1}, f_{2}, \ldots, f_{n}\right)=\operatorname{mdc}\left(f_{1}, \operatorname{mdc}\left(f_{2}, \ldots, f_{n}\right)\right)$, de forma que podemos encontrar $h$ por meio de sucessivạs aplicações do Algoritmo de Euclides.

Um comentário importante antes de encerrarmos esta seção é que utilizamos, a grosso modo, estratégias muito parecidas para resolver a pertinência nos contextos de espaços vetoriais e $K[x]$. Em ambos os casos, primeiro encontramos um conjunto gerador especial do ideal (ou subespaço) em questão. Com esse conjunto em mãos, pudemos realizar alguma espécie de "divisão" e a pertinência foi dada de forma imediata pelo resto nessa divisão. Adiante, veremos que esses conjuntos geradores especiais (no caso de ideais) são justamente as Bases de Gröbner.

Encerramos a seção com um exemplo que ilustra o procedimento descrito acima, verificando a pertinência do polinômio $p$ no ideal $I$.

Exemplo 2.3.1 Seja $p:=x^{3}+4 x^{2}+3 x-7$ um polinômio de $\mathbb{Q}[x]$ e

$$
I:=\left\langle f_{1}:=x^{3}-3 x+2, f_{2}:=x^{4}-1, f_{3}:=x^{6}-1\right\rangle
$$

um ideal de $\mathbb{Q}[x]$.

Utilizando o Algoritmo de Euclides, obtemos:

$\operatorname{mdc}\left(f_{1}, f_{2}, f_{3}\right)=\operatorname{mdc}\left(f_{1}, \operatorname{mdc}\left(f_{2}, f_{3}\right)\right)=\operatorname{mdc}\left(f_{1}, x^{2}-1\right)=-2 x+2$, donde $I=$ $\langle-2 x+2\rangle$.

Por fim, ao aplicarmos o Algoritmo da Divisão para dividirmos $p$ por $-2 x+2$, obtemos resto 2 , o que nos mostra que $p$ não pertence a $I$.

\subsection{Bases de Gröbner Comutativas}

Nesta seção apresentamos os principais conceitos e resultados envolvendo a Teoria de Bases de Gröbner comutativas. Contudo, uma vez que o foco do restante do trabalho será o caso não 
comutativo, não temos a pretensão de mostrar essa teoria em profundidade nem dar a ela todo espaço que merece, deixando tal aprofundamento para outras referências no assunto [Buc65, BW93, CLO97, AL94, vzGG99]. Nossa exposição seguirá de perto a de Cox, Little e O'Shea [CLO97].

Ao longo desta seção, trabalharemos com o anel de polinômios comutativo em $n$ variáveis: $K\left[x_{1}, \ldots, x_{n}\right]$. Utilizaremos bastante o fato de que, dada uma ordem nas variáveis, podemos escrever um monômio como um elemento de $\mathbb{N}^{n}$. Assumiremos aqui que tal ordem é $x_{1}>x_{2}>\cdots>x_{n}$ e escreveremos os monômios sempre em ordem decrescente, como ilustrado nos exemplos que seguem. Dado um elemento $\alpha$ de $\mathbb{N}^{n}$, chamaremos de $\alpha_{i}$ sua $i$-ésima coordenada e denotaremos então um monômio $x_{1}^{\alpha_{1}} x_{2}^{\alpha_{2}} \cdots x_{n}^{\alpha_{n}}$ por $x^{\left(\alpha_{1}, \alpha_{2}, \ldots, \alpha_{n}\right)}=x^{\alpha}$. Por exemplo, $x_{1}^{2} x_{3} x_{5}^{6} \in K\left[x_{1}, x_{2}, \ldots, x_{6}\right]$ pode ser escrito como $x^{(2,0,1,0,6,0)}$ (ou simplesmente como $(2,0,1,0,6,0)$ ), $x_{1} x_{2}^{3} x_{6}$ como $x^{(1,3,0,0,0,1)}$ (ou $(1,3,0,0,0,1))$ e $x_{1}^{2} x_{3} x_{5}^{6} \cdot x_{1} x_{2}^{3} x_{6}$ como $x^{(2,0,1,0,6,0)+(1,3,0,0,0,1)}$ (ou $(2,0,1,0,6,0)+(1,3,0,0,0,1)$ ). Consideramos ainda a unidade de $K\left[x_{1}, \ldots, x_{n}\right], 1$, como o monômio $x^{(0,0, \ldots, 0)}$ e chamamos a atenção do leitor para o fato de que os monômios formam uma base (infinita) para $K\left[x_{1}, \ldots, x_{n}\right]$ como espaço vetorial.

Iniciamos a seção com um conceito fundamental em Teoria de Bases de Gröbner. Como vimos nas seções anteriores, as soluções apresentadas para o problema da pertinência em espaços vetoriais de dimensão finita e em $K[x]$ exigiram a presença de uma ordem especial. Aqui também precisaremos de uma ordem nos monômios de $K\left[x_{1}, \ldots, x_{n}\right]$ que satisfaça nossos propósitos.

Definição 2.4.1 (Ordem monomial) Uma ordem monomial em $K\left[x_{1}, \ldots, x_{n}\right]$ é uma relação $\leq$ nos monômios de $K\left[x_{1}, \ldots, x_{n}\right]$, satisfazendo:

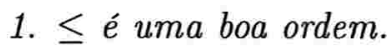

2. Para todos os monômios $x^{\alpha}, x^{\beta}, x^{\gamma} \in K\left[x_{1}, \ldots, x_{n}\right]$, se $x^{\alpha}<x^{\beta}$ então $x^{\alpha} x^{\gamma}<x^{\beta} x^{\gamma}$.

Em particular, a segunda condição da definição nos diz que se temos uma seqüência de monômios ordenada por uma ordem monomial, ao multiplicarmos todos eles por um mesmo monômio, a relação de ordem original será preservada. Uma conseqüência importante dessa condição é que se escrevemos um polinômio como soma de seus termos agrupados e ordenados por uma ordem monomial (existe uma única maneira de escrever um polinômio dessa forma) e o multiplicamos por um monômio, não é necessário reordenar os termos após a multiplicação.

Seguem alguns exemplos de ordens monomiais em $K\left[x_{1}, \ldots, x_{n}\right]$. Assumimos que $x^{\alpha}$ e $x^{\beta}$ são dois monômios de $K\left[x_{1}, \ldots, x_{n}\right] \operatorname{com} \alpha=\left(\alpha_{1}, \alpha_{2}, \ldots, \alpha_{n}\right)$ e $\beta=\left(\beta_{1}, \beta_{2}, \ldots, \beta_{n}\right)$.

Ordem lexicográfica (abreviada por lex) Dizemos que $x^{\alpha}<x^{\beta}$ se existe $1 \leq j \leq n$ tal que $\alpha_{j}<\beta_{j}$ e $\alpha_{i}=\beta_{i}$, para todo $1 \leq i<j .{ }^{5}$ Como exemplos, podemos citar:

$$
\begin{aligned}
& x_{1} x_{2}^{2} x_{3} x_{4}<x_{1} x_{2}^{3} x_{3} \\
& x_{1} x_{3}^{10}<x_{1} x_{2} x_{3}^{2} \\
& x_{1}>x_{2}>\cdots>x_{n}
\end{aligned}
$$

Ordem de grau e lexicografia (abreviada por deglex) Dizemos que $x^{\alpha}<x^{\beta}$ se

\footnotetext{
${ }^{5}$ Note que a ordem lexicográfica depende da ordem definida previamente nas variáveis e da forma como os monòmios são escritos como elementos de $\mathbb{N}^{n}$. Ainda, a ordem lexicográfica é compatível com a ordem que assumimos inicialmente para as variáveis.
} 
$\sum_{i=1}^{n} \alpha_{i}<\sum_{i=1}^{n} \beta_{i}$ ou

$\sum_{i=1}^{n} \alpha_{i}=\sum_{i=1}^{n} \beta_{i}$ e $x^{\alpha}$ é menor que $x^{\beta}$ na ordem lexicográfica.

Podemos citar os seguintes exemplos:

$$
\begin{aligned}
& x_{1} x_{2} x_{3}^{2}<x_{1} x_{3}^{10} \\
& x_{1} x_{2} x_{3}<x_{2}^{5} \\
& x_{2}^{2}<x_{1}^{2} \\
& x_{1}>x_{2}>\cdots>x_{n}
\end{aligned}
$$

Ordem lexicográfica reversa (abreviada por revlex) Dizemos que $x^{\alpha}<x^{\beta}$ se existe $1 \leq j \leq$ $n$ tal que $\alpha_{j}<\beta_{j}$ e $\alpha_{i}=\beta_{i}$, para todo $j<i \leq n$. Como exemplos, podemos citar:

$$
\begin{aligned}
& x_{1} x_{2}^{2} x_{3} x_{4}>x_{1} x_{2}^{3} x_{3} \\
& x_{1} x_{3}^{10}>x_{1} x_{2} x_{3}^{2} \\
& x_{1}<x_{2}<\cdots<x_{n}
\end{aligned}
$$

Uma observação bastante interessante é que, tal qual a ordem lexicográfica, a ordem revlex também depende da ordem inicial escolhida para as variáveis e da forma como escrevemos os monômios como tuplas de $\mathbb{N}^{n}$.

Porém, revlex não é compatível com a ordem que demos para as variáveis no início da seção a fim de representar os monômios como tuplas ordenadas de inteiros não negativos. Isso não representa qualquer tipo de problema para a ordem revlex. De fato, a ordem escolhida inicialmente nas variáveis tem apenas o propósito de representar monômios de uma maneira conveniente, mas para decidirmos qual monômio é menor que outro, utilizamos sempre a ordem monomial fixada.

O leitor interessado é ainda convidado a verificar o que ocorre com as ordens lexicográficas se invertermos a ordem escolhida nas variáveis e escrevermos as tuplas em ordem crescente. Ao longo do texto, seguiremos com a maneira definida no início da seção por ser a mais natural.

Ordem de grau e lexicografia reversa (abreviada por degrevlex) Dizemos que $x^{\alpha}<x^{\beta}$ se

$$
\begin{aligned}
& \sum_{i=1}^{n} \alpha_{i}<\sum_{i=1}^{n} \beta_{i} \text { ou } \\
& \sum_{i=1}^{n} \alpha_{i}=\sum_{i=1}^{n} \beta_{i} \text { e } x^{\alpha}<x^{\beta} \text { de acordo com a ordem lexicográfica reversa. }
\end{aligned}
$$

Seguem alguns exemplos de monômios ordenados por esta ordem.

$$
\begin{aligned}
& x_{1} x_{2}^{2} x_{3}<x_{1} x_{2} x_{3}^{2} \\
& x_{1} x_{2}^{2}<x_{1} x_{3}^{2} \\
& x_{1}<x_{2}<\cdots<x_{n}
\end{aligned}
$$

Deixamos a cargo do leitor verificar que essas ordens são, de fato, monomiais.

Daqui por diante assumiremos que está fixada uma ordem monomial em $K\left[x_{1}, \ldots, x_{n}\right]$. Além disso, escreveremos os polinômios sempre com seus termos em ordem decrescente (de acordo com a ordem monomial sendo utilizada). 
Definição 2.4.2 (Monômio Líder) Seja $f=a_{1} x^{\alpha_{1}}+a_{2} x^{\alpha_{2}}+\cdots+a_{k} x^{\alpha_{k}}$ um polinômio não nulo de $K\left[x_{1}, \ldots, x_{n}\right]$, com $0 \neq a_{i} \in K$ e $\alpha_{i} \in \mathbb{N}^{n}$, para $1 \leq i \leq k$ e $\leq$ uma ordem monomial em $K\left[x_{1}, \ldots, x_{n}\right]$. Tome $x^{\alpha_{j}}$ o máximo dentre todos os $x^{\alpha_{i}}$, na ordem $\leq$.

Chamamos $x^{\alpha_{j}}$ o monômio líder de $f$ (na ordem dada), e o denotamos por $\widehat{f}$.

Ainda, chamamos $a_{j}$ de coeficiente lider de $f$ e o denotamos por $c l(f)$.

Por fim, se $S$ é um subconjunto de $K\left[x_{1}, \ldots, x_{n}\right]$, chamamos $\widehat{S}$ o conjunto $\{\widehat{h} \mid h \in S\}$.

Com essa definição, podemos apresentar o Algoritmo da Divisão (Algoritmo 2.4.1), que realiza para Teoria de Bases de Gröbner um papel análogo ao que realizaram a Redução Incompleta de Gauss e o Algoritmo da Divisão em $K[x]$ nas seções anteriores. Conforme veremos na Seção 4.1, esse algoritmo pode ser adaptado praticamente sem alterações para o caso não comutativo, de forma que adiaremos o estudo dos detalhes de seu funcionamento para o Capítulo 4.

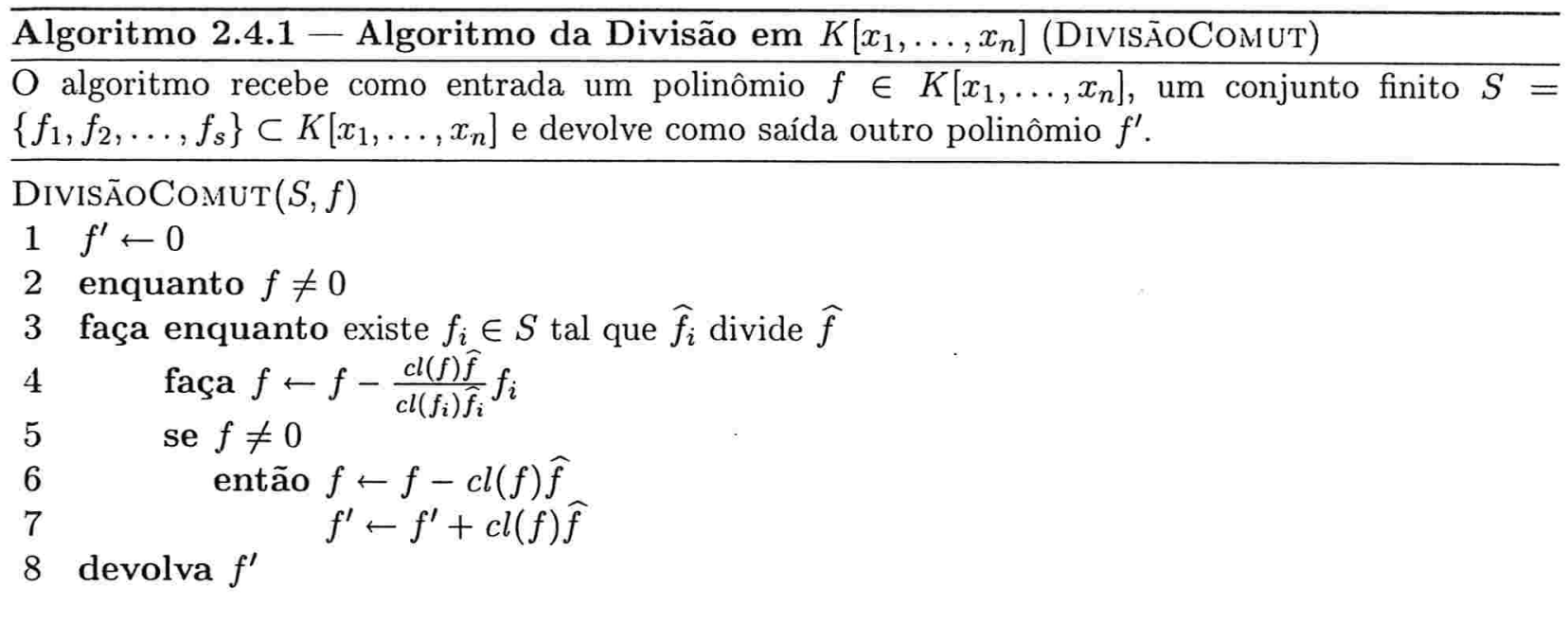

Dados um polinômio $f$ em $K\left[x_{1}, \ldots, x_{n}\right]$ e um subconjunto finito $S$ de $K\left[x_{1}, \ldots, x_{n}\right]$, se $f^{\prime}:=$ $\operatorname{DivisãoComut~}(f, S)$, diremos que $f$ se reduz para $f^{\prime}$ sobre $S$ (denotado por $f \Rightarrow_{S} f^{\prime}$ ). Além disso, dado um polinômio $p$ tal que $\widehat{p}$ divide $\widehat{f}$, chamaremos o elemento $f-\frac{c l(f) \widehat{f}}{c l(p) \hat{p}} p$ de redução simples de $f$ por $p$ (note que o lado direito de cada atribuição da linha 4 do algoritmo é uma redução simples de $f$ por $f_{i}$ ).

Como comentado acima, adiaremos maiores análises do algoritmo para sua versão não comutativa. Porém, vale comentar que, a cada iteração, o monômio líder de $f$ se torna menor, o que garante que o processo descrito acima pára em um número finito de passos, devido à presença de uma boa ordem.

Apresentamos um exemplo a seguir que ilustra o importante fato de que a saíla do Algoritmo da Divisão depende da ordem em que os elementos do conjunto $S$ são considerados. Veremos mais adiante que a ferramenta necessária para eliminar essa característica indesejável do algoritmo são as Bases de Gröbner.

Exemplo 2.4.1 Considere $K[x, y, z]$ com a ordem lexicográfica $(x>y>z)$. Tome $S=\left\{f_{1}:=x^{2}-y^{3} z, f_{2}:=x^{2}-z^{2}, f_{3}:=y^{2} z-y\right\}$ e $f:=x^{2}-y^{2}$. 
Se o algoritmo escolhe $f_{1}$ para a primeira iteração teremos, na linha $4, f \leftarrow y^{3} z-y^{2}$. Para a segunda iteração, resta então uma única opção: $f_{3}$, donde $f \leftarrow 0$ e o algoritmo devolverá 0 .

Por outro lado, se o algoritmo escolher $f_{2}$ na primeira iteração, a atribuição realizada nesta iteração pela linha 4 será $f \leftarrow-y^{2}+z^{2}$. Mas então, como nem $-y^{2}$ nem $z^{2}$ são divisíveis pelo monômio líder de nenhum elemento de $S$, segue que a saída do algoritmo será $-y^{2}+z^{2}$.

Apresentamos em seguida um resultado bastante conhecido, que nos dará uma importante propriedade sobre ideais monomiais de $K\left[x_{1}, \ldots, x_{n}\right]$, o Lema de Dickson. Lembramos que um ideal $I$ de $K\left[x_{1}, \ldots, x_{n}\right]$ é monomial se existe um conjunto (possivelmente infinito) $A \subset \mathbb{N}^{n}$ tal que $I=\left\langle\left\{x^{\alpha} \mid \alpha \in A\right\}\right\rangle$.

Teorema 2.4.1 (Lema de Dickson) Dado um ideal monomial de $K\left[x_{1}, \ldots, x_{n}\right]$, da forma $I=$ $\left\langle\left\{x^{\alpha} \mid \alpha \in A\right\}\right\rangle$, com $A$ um subconjunto (possivelmente infinito) de $\mathbb{N}^{n}$, existe um subconjunto finito $B$ de $A$ tal que $I=\left\langle\left\{x^{\beta} \mid \beta \in B\right\}\right\rangle$. Em particular, segue que todo ideal monomial de $K\left[x_{1}, \ldots, x_{n}\right]$ é finitamente gerado.

Demonstração. Tome $A$ um subconjunto (possivelmente infinito) de $\mathbb{N}^{n}$ e seja $I=$ $\left\langle\left\{x^{\alpha} \mid \alpha \in A\right\}\right\rangle$. Mostraremos que $I$ possui um conjunto gerador monomial finito. A prova segue por indução em $n$ (o número de variáveis). Se $n=1$, então os elementos de $A$ podem ser vistos como inteiros maiores ou iguais a zero, de forma que podemos tomar o menor elemento de $A$ (denote-o por $m$ ). Claramente, $x^{m}$ gera $I$ neste caso.

Supomos agora que o resultado é verdadeiro para $K\left[x_{1}, x_{2}, \ldots, x_{l}\right]$, para todo $l<n$ e vejamos que ele é verdadeiro para $K\left[x_{1}, \ldots, x_{n}\right]$. Consideramos então o seguinte homomorfismo de álgebras, dado por sua imagem nos monômios:

$$
\begin{array}{ll}
\varphi: & K\left[x_{1}, \ldots, x_{n}\right] \longrightarrow K\left[x_{1}, x_{2}, \ldots, x_{n-1}\right] \\
& x^{\left(\alpha_{1}, \alpha_{2}, \ldots, \alpha_{n-1}, \alpha_{n}\right)} \mapsto x^{\left(\alpha_{1}, \alpha_{2}, \ldots, \alpha_{n-1}\right)}
\end{array}
$$

Em outras palavras, $\varphi$ preserva os monômios $x_{1}, \ldots, x_{n-1}$ e leva $x_{n}$ em 1 .

Claro que $\varphi(I)$ é um ideal monomial de $K\left[x_{1}, x_{2}, \ldots, x_{n-1}\right]$. Então, por hipótese de indução, $\varphi(I)$ é finitamente gerado, digamos, por $x^{\beta^{1}}, x^{\beta^{2}}, \ldots, x^{\beta^{k}}$. Considere então, para cada $1 \leq i \leq k, \varphi^{-1}\left(x^{\beta^{i}}\right)$. Pela definição de $\varphi$, temos que, para cada $1 \leq i \leq k$ existe um inteiro $m^{i} \geq 0$ com a propriedade de que $x^{\left(\beta_{1}^{i}, \beta_{2}^{i}, \ldots, \beta_{n-1}^{i}, m^{i}\right)}$ pertence a $I$ (em particular pertence a $\left.\varphi^{-1}\left(x^{\beta^{i}}\right)\right)$, uma vez que $x^{\beta^{i}} \in \varphi(I)$. Escolha então, para cada $1 \leq i \leq k$ um tal valor de $m^{i}$ e denote por $m$ o maior inteiro dentre esses números. Vamos então construir um conjunto gerador finito para $I$.

Para tanto, para cada inteiro $0 \leq j \leq m-1$, definimos por $I_{j}$ o subconjunto de $I$ formado pelos elementos da forma $x^{\left(\alpha_{1}, \alpha_{2}, \ldots, \alpha_{n-1} \cdot j\right)}$, ou seja, $I_{j}$ é o subconjunto de $I$ composto pelos monômios cuja coordenada $n$ é exatamente $j$. Note ainda que, para cada $j, \varphi\left(I_{j}\right)$ é um ideal monomial de $K\left[x_{1}, x_{2}, \ldots, x_{n-1}\right]$. 
Logo, por hipótese de indução, cada $\varphi\left(I_{j}\right)$ possui um conjunto gerador monomial finito. Denotemos por $\left\{\beta(j)^{1}, \beta(j)^{2}, \ldots, \beta(j)^{k_{j}}\right\}$ tal conjunto gerador. Sejam então: ${ }^{6}$

$$
\begin{aligned}
& J:=\left\{x^{\beta^{1}} x_{n}^{m}, x^{\beta^{2}} x_{n}^{m}, \ldots, x^{\beta^{k}} x_{n}^{m}\right\} \\
& J_{j}:=\left\{x^{\beta(j)^{1}} x_{n}^{j}, x^{\beta(j)^{2}} x_{n}^{j}, \ldots, x^{\beta(j)^{k_{j}}} x_{n}^{j}\right\}, \text { para } 1 \leq j \leq m-1
\end{aligned}
$$

Denotamos por

$$
B=\cup_{j=1}^{m-1} J_{j} \cup J
$$

Afirmamos que $B$ é uma conjunto gerador para $I$ (ele é claramente finito). Primeiramente, note que $B$ está contido em $I$ por construção. Mostraremos então que todo monômio $x^{\alpha}$ de $I$ é divisível por algum monômio em $B$. Mas, se $\alpha_{n} \geq m$, segue que $x^{\alpha}$ é divisivel por algum monômio de $J$. Senão, $\alpha_{n}$ é um valor entre 0 e $m-1$ e $x^{\alpha}$ será divisível por algum monômio de $J_{\alpha_{n}}$.

Para encerrar a demonstração, resta mostrar que podemos encontrar um subconjunto finito de $A$ que gera $I$. Tome então um elemento $x^{\beta}$ de $B$. Como $x^{\beta}$ pertence a $I$, segue que ele é divisível por algum $x^{\alpha}$, $\operatorname{com} \alpha$ em $A$. Portanto, podemos escolher finitos elementos de $A$ que geram $I$.

Em seguida apresentamos uma versão do Teorema da Base de Hilbert, que tem como conseqüência o fato de $K\left[x_{1}, \ldots, x_{n}\right]$ ser noetheriano. Esse resultado é bastante famoso e tem importância central tanto em Teoria de Bases de Gröbner comutativas, quanto em Teoria de Anéis comutativos em geral.

Vale ressaltar que na demonstração aqui apresentada alguns conceitos importantes da Teoria Bases de Gröbner serão utilizados e a própria definição dessas bases surgirá de maneira natural. Ademais, a prova aqui apresentada é construtiva, diferentemente da demonstração original desse teorema. Além disso, o fato da Teoria de Bases de Gröbner ser utilizada para demonstrar um resultado tão central serve como um indício de sua importância.

Teorema 2.4.2 (Teorema da Base de Hilbert) Todo ideal $I$ de $K\left[x_{1}, \ldots, x_{n}\right]$ é finitamente gerado, ou seja $I=\left\langle g_{1}, g_{2}, \ldots, g_{k}\right\rangle$, para alguns $g_{1}, g_{2}, \ldots, g_{k} \in I$.

Demonstração. Primeiramente, se $I=\{0\}$ não há o que fazer. Assumimos então que existe um polinômio não nulo em $I$ e consideramos o ideal $\langle\widehat{I}\rangle$, que é monomial por definição. Pelo Lema de Dickson (Teorema 2.4.1), $\langle\widehat{I}\rangle$ é finitamente gerado por monômios, i.e. $\langle\widehat{I}\rangle=\left\langle b_{1}, b_{2}, \ldots, b_{k}\right\rangle$. Porém, como cada $b_{i}$ pertence a $\langle\widehat{I}\rangle$ e é um monômio, segue que $b_{i}=x^{\alpha} \widehat{f}_{i}$, para algum $\widehat{f}_{i} \in \widehat{I}$. Com isso, temos que existem polinômios $f_{1}, f_{2}, \ldots f_{k}$ em $I$ tais que $\langle\widehat{I}\rangle=\left\langle\widehat{f}_{1}, \widehat{f}_{2}, \ldots, \widehat{f}_{k}\right\rangle$. Mostraremos que $\left\{f_{1}, f_{2}, \ldots f_{k}\right\}$ constitui um conjunto gerador para $I$ (naturalmente esse conjunto é finito).

Por um lado, $f_{1}, f_{2}, \ldots f_{k}$ pertencem a $I$, de forma que $\left\langle f_{1}, f_{2}, \ldots f_{k}\right\rangle \subseteq I$. Resta verificar que $I \subseteq\left\langle f_{1}, f_{2}, \ldots f_{k}\right\rangle$. Assumimos por contradição que isso não é verdade, ou seja, que

\footnotetext{
${ }^{6}$ Cometemos aqui um pequeno abuso de notação e identificamos o elemento $x^{\left(\alpha_{1}, \alpha_{2}, \ldots \ldots \alpha_{n-1}\right)}$ de $K\left[x_{1}, x_{2}, \ldots, x_{n-1}\right]$ com o elemento $x^{\left(\alpha_{1}, \alpha_{2}, \ldots, \alpha_{n-1}, 0\right)}$ de $K\left[x_{1}, \ldots, x_{n}\right]$.
} 
existe um conjunto $A \subseteq I$ não vazio formado pelos elementos que não pertencem ao ideal gerado por $f_{1}, f_{2}, \ldots f_{k}$.

Mas então, pela presença de uma ordem monomial, temos que o conjunto $\widehat{A}$ possui um menor elemento. Tome então um polinômio $p \in A$, tal que $\widehat{p}$ é mínimo em $\widehat{A}$, ou seja, qualquer outro polinômio com monômio líder menor (se existir) não pertence a $A$. Por outro lado, $\widehat{p} \in \widehat{I}$, logo $\widehat{p}$ é divisível por $\widehat{f}_{i}$ (digamos que $\widehat{p}=x^{\alpha} \widehat{f}_{i}$ ), para algum $1 \leq i \leq k$. Podemos então escrever

$$
h=p-\frac{c l(p)}{c l\left(f_{i}\right)} x^{\alpha} f_{i}
$$

Com isso, $h \in I$ e $\widehat{h}<\widehat{p}$, donde $h \in\left\langle f_{1}, f_{2}, \ldots f_{k}\right\rangle$ (pela minimalidade de $\widehat{p}$ ) ou $h=0$. Mas então $p$ também pertence a $\left\langle f_{1}, f_{2}, \ldots f_{k}\right\rangle$, uma contradição.

Logo, $I \subseteq\left\langle f_{1}, f_{2}, \ldots f_{k}\right\rangle$ e concluímos que $I=\left\langle f_{1}, f_{2}, \ldots f_{k}\right\rangle$, o que encerra a demonstração.

O sucesso da demonstração apresentada para o Teorema da Base de Hilbert faz uso de uma propriedade fundamental do conjunto $\left\{f_{1}, f_{2}, \ldots f_{k}\right\}:\left\langle\widehat{f}_{1}, \widehat{f}_{2}, \ldots, \widehat{f}_{k}\right\rangle=\langle\widehat{I}\rangle$, ou equivalentemente, para todo polinômio $h$ não nulo de $I$, existe $f_{i},(1 \leq i \leq k)$ tal que $f_{i}$ divide $\widehat{h}$. Essa é exatamente a propriedade chave das Bases de Gröbner.

Definição 2.4.3 (Base de Gröbner) Um subconjunto $G$ de um ideal $I$ de $K\left[x_{1}, \ldots, x_{n}\right]$ é uma Base de Gröbner para I (na ordem monomial fixada) se $\langle\widehat{G}\rangle=\langle\widehat{I}\rangle$, ou equivalentemente se, para todo polinômio não nulo $f \in I$, existe um polinômio $g \in G$ tal que $\widehat{g}$ divide $\widehat{f}$.

Chamamos a atenção do leitor para o fato de que o conjunto $G$ presente na definição acima pode ser infinito.

Uma observação interessante é que todo ideal não nulo possui uma Base de Gröbner (todo ideal é uma Base de Gröbner para si mesmo). Além disso, uma pequena modificação na demonstração do Teorema 2.4.2 nos mostra que uma Base de Gröbner para um ideal $I$ gera $I$ (como ideal). Com isso, freqüentemente nos referiremos apenas a uma Base de Gröbner $G$, o que quer dizer que $G$ é uma Base de Gröbner para o ideal gerado por ela.

Vale observar ainda que, apesar de seu nome, uma Base de Gröbner para um ideal $I$ não é uma base de $I$ como espaço vetorial (em geral não é nem mesmo um conjunto gerador como espaço vetorial). A fim de ilustrar a definição acima, apresentamos um exemplo:

Exemplo 2.4.2 Considere $K[x, y]$ com a ordem de grau e lexicografia $(x>y)$. Tome $F=\left\{f_{1}, f_{2}\right\}$ e $I$ o ideal gerado por $F$, onde $f_{1}=x^{3}-2 x y$ e $f_{2}=x^{2} y-2 y^{2}+x$. Então o conjunto $F$ não é uma Base de Gröbner para $I$. De fato, $f_{1} y-f_{2} x=-x^{2} \in I$, mas $-x^{2} \notin\left\langle\widehat{f}_{1}, \widehat{f}_{2}\right\rangle$.

Adiante nesta seção, veremos como construir Bases de Gröbner a partir de um conjunto gerador. Por ora, podemos citar que o conjunto $\left\{x^{3}-2 x y, x^{2} y-2 y^{2}+x, x^{2}, 2 x y, 2 y^{2}-x\right\}$ é uma Base de Gröbner para $I$ (na realidade, o conjunto $\left\{x^{2}, 2 x y, 2 y^{2}-x\right\}$ também é uma Base de Gröbner para $I$, embora ele não contenha nem $f_{1}$ nem $f_{2}$ ). 
O seguinte resultado fornece uma das propriedades mais importantes das Bases de Gröbner e justifica a observação sobre elas feita logo após a apresentação do Algoritmo da Divisão para polinômios comutativos.

Proposição 2.4.3 Seja $G$ uma Base de Gröbner de um ideal $I$ de $K\left[x_{1}, \ldots, x_{n}\right]$ e seja $f \in$ $K\left[x_{1}, \ldots, x_{n}\right]$ um polinômio. Então existe um único polinômio $f^{\prime} \in K\left[x_{1}, \ldots, x_{n}\right]$ tal que:

1. Nenhum termo de $f^{\prime}$ é divisivel por um elemento do conjunto $\widehat{G}$.

2. Existe $g \in I$ tal que $f=g+f^{\prime}$.

Em particular, essa proposição nos garante que a saída do Algoritmo da Divisão ao se utilizar uma Base de Gröbner independe da ordem em que os elementos dessa base são escolhidos durante as reduções feitas pelo algoritmo. Outra conseqüência importante é o seguinte corolário.

Corolário 2.4.4 Seja G uma Base de Gröbner de um ideal I de $K\left[x_{1}, \ldots, x_{n}\right]$ e seja $f$ um polinômio em $K\left[x_{1}, \ldots, x_{n}\right]$. Então $f^{\prime}:=\operatorname{DivisÃoComut~}(G, f)$ é igual a zero se e somente se $f$ pertence a $I$.

Demonstrações para os análogos desses dois últimos resultados no contexto não comutativo serão apresentadas adiante. Omitimo-nas daqui pelo caráter superficial desta seção e pelo fato de que as demonstrações feitas no caso não comutativo são facilmente adaptáveis para cá. Ilustramos a importância desses resultados com um exemplo de sua aplicação para resolver uma instância do problema da pertinência, dada uma Base de Gröbner para o ideal de interesse.

Exemplo 2.4.3 Tome $K[x, y]$ com a ordem de grau e lexicografia $(x>y)$ e $G=$ $\left\{x^{2}, 2 x y, 2 y^{2}-x\right\}$ a Base de Gröbner vista no exemplo anterior. Seja ainda $f=x^{2} y-$ $2 y^{2}+x$; vamos utilizar o Algoritmo da Divisão para mostrar que $f \in\langle G\rangle$. Exibimos a seqüência de reduções realizada pelo algoritmo: ${ }^{7}$

O algoritmo escolhe $x^{2}: f \leftarrow x^{2} y-2 y^{2}+x-x^{2} y=-2 y^{2}+x$

O algoritmo escolhe $2 y^{2}-x: f \leftarrow 0$ e o algoritmo pára, devolvendo 0 como saída, donde se conclui que $f$ pertence ao ideal $\langle G\rangle$.

Voltamo-nos agora para a importante questão de como encontrar Bases de Gröbner para um ideal $I$ a partir de um conjunto gerador $F$. Intuitivamente, o conjunto $F$ pode não conter todos os monômios líderes de $I$, pois podem ocorrer cancelamentos de monômios líderes, como visto no Exemplo 2.4.2. A fim de transformar $F$ em uma Base de Gröbner, precisamos do conceito de S-polinômio.

Definição 2.4.4 (S-Polinômio) Sejam $f, g$ polinômios de $K\left[x_{1}, \ldots, x_{n}\right]$. O S-polinômio de $f e$ $g$ é

\footnotetext{
${ }^{7}$ Note que mais de uma seqüência de reduções é possivel. Entretanto, de acordo com a Proposição 2.4.3, pelo fato de termos uma Base de Gröbner em mãos, diferentes sequiências de reduçōes não alteram a saída do algoritmo. $O$ leitor cético é convidado a examinar a saída do algoritmo para uma sequêencia diferente de reduçōes da apresentada neste exemplo.
} 


$$
S(f, g):=\frac{x^{\gamma}}{c l(f) \widehat{f}} f-\frac{x^{\gamma}}{c l(g) \widehat{g}} g
$$

onde $x^{\gamma}$ é o minimo múltiplo comum de $\widehat{f}$ e $\widehat{g}$, ou seja, se $\widehat{f}=x^{\alpha}$ e $\widehat{g}=x^{\beta}$, com $\alpha=\left(\alpha_{1}, \alpha_{2}, \ldots, \alpha_{n}\right)$ e $\beta=\left(\beta_{1}, \beta_{2}, \ldots, \beta_{n}\right)$, então $\gamma=\left(\gamma_{1}, \gamma_{2}, \ldots, \gamma_{n}\right)$, com $\gamma_{i}=\max \left(\left\{\alpha_{i}, \beta_{i}\right\}\right)$, para $1 \leq i \leq n$.

Os S-polinômios são projetados para que ocorra o cancelamento de monômios líderes; se retomarmos o Exemplo 2.4.2, teremos:

$$
S\left(f_{1}, f_{2}\right)=y f_{1}-x f_{2}=-x^{2}
$$

O seguinte teorema é de fundamental importância para encontrarmos Bases de Gröbner para um ideal a partir de seus geradores e nos fornece um critério para decidir quando um conjunto gerador é uma Base de Gröbner.

Teorema 2.4.5 Seja I um ideal de $K\left[x_{1}, \ldots, x_{n}\right]$. Então um conjunto gerador $G=\left\{g_{1}, g_{2}, \ldots, g_{k}\right\}$ de I é uma Base de Gröbner para $I$ se e somente se para todos os pares $(i, j)$, com $i \neq j, S\left(g_{i}, g_{j}\right)$ se reduz para zero sobre $G$ (para alguma ordem em que os elementos de $G$ são considerados pelo Algoritmo da Divisão).

Com esse resultado, podemos apresentar o Algoritmo de Buchberger, que constrói uma Base de Gröbner para um ideal $I$ a partir de um conjunto gerador $F$.

A idéia básica do algoritmo é considerar cada S-polinômio entre elementos de $F$. Então, para cada um deles, o algoritmo calcula sua redução sobre $F$ e a adiciona em $F$, caso ela não seja nula.

Se tomarmos como exemplo o S-polinômio visto acima, o algoritmo incluiria $-x^{2}$ em $F$ e, com isso, esse S-polinômio passaria a se reduzir para zero sobre $F$ (pelo menos para alguma sequiência de reduções). Uma descrição do Algoritmo de Buchberger pode ser vista no Algoritmo 2.4.2.

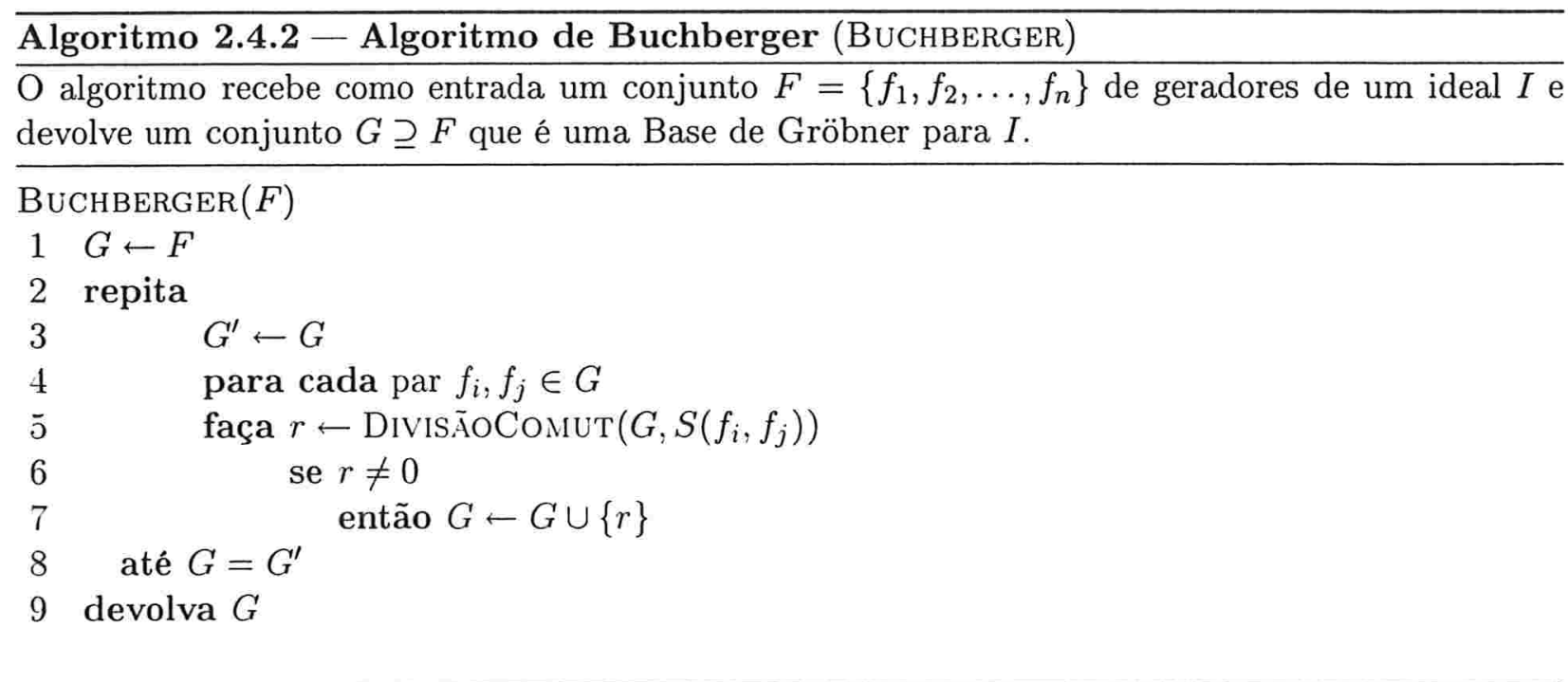

Mostramos então como esse algoritmo pode ser utilizado para encontrar uma Base de Gröbner para o ideal do Exemplo 2.4.2. 
Exemplo 2.4.4 Sejam $F$ e $I$ como no Exemplo 2.4.2. O Algoritmo de Buchberger trata os seguintes S-polinômios.

$$
\begin{aligned}
& S\left(f_{1}, f_{2}\right)=-x^{2} \Rightarrow_{F}-x^{2}, \text { e } F \leftarrow F \cup\left\{f_{3}:=-x^{2}\right\} \\
& S\left(f_{1}, f_{3}\right)=-2 x y \Rightarrow_{F}-2 x y, \text { e } F \leftarrow F \cup\left\{f_{4}:=-2 x y\right\} \\
& S\left(f_{2}, f_{3}\right)=-2 y^{2}+x \Rightarrow_{F}-2 y^{2}+x, \text { e } F \leftarrow F \cup\left\{f_{5}:=-2 y^{2}+x\right\} \\
& S\left(f_{1}, f_{4}\right)=-2 x y^{2} \Rightarrow_{F} 0
\end{aligned}
$$

Os demais S-polinômios envolvendo elementos de $F$ também se reduzem todos para zero sobre $F$. Logo, o conjunto $G$ não é mais modificado e o algoritmo pára, devolvendo o conjunto $\left\{f_{1}, f_{2}, f_{3}, f_{4}, f_{5}\right\}$, que é de fato uma Base de Gröbner para $I$, como comentado no Exemplo 2.4.2. A

O leitor interessado encontra demonstrações de correção e outros comentários em referências voltadas para o caso comutativo [Buc65, CLO97, BW93]. Limitamo-nos a mencionar que o Teorema da Base de Hilbert tem uma importância fundamental na demonstração de que o processo acima pára, tendo como conseqüência que todo ideal de $K\left[x_{1}, \ldots, x_{n}\right]$ possui uma Base de Gröbner finita (e que pode ser encontrada pelo Algoritmo de Buchberger). Destacamos esse importante fato no seguinte teorema.

Teorema 2.4.6 Seja $F=\left\{f_{1}, f_{2}, \ldots, f_{n}\right\}$ o conjunto gerador de um ideal $I$ não nulo do anel de polinômios comutativo, $K\left[x_{1}, \ldots, x_{n}\right]$. Se $F$ for fornecido como entrada para o Algoritmo de Buchberger, esse terminará em um número finito de passos e devolverá uma Base de Gröbner finita para $I$.

Destacamos que o Algoritmo de Buchberger constitui o elemento final para resolver o problema da pertinência em $K\left[x_{1}, \ldots, x_{n}\right]$. De fato, para resolver a pertinência em $K\left[x_{1}, \ldots, x_{n}\right]$, basta encontrar uma Base de Gröbner para o ideal de interesse por meio do Algoritmo de Buchberger e então utilizar o Algoritmo da Divisão, à luz do que foi feito no Exemplo 2.4.3.

Lembramos ainda que, conforme mencionado na Seção 2.1, esse processo fornece uma solução para o problema da igualdade em quocientes de $K\left[x_{1}, \ldots, x_{n}\right]$. Com efeito, dado um conjunto gerador para um ideal $I$ de $K\left[x_{1}, \ldots, x_{n}\right]$, para decidir se $f+I=g+I$, basta encontrar uma Base de Gröbner para $I$ com o Algoritmo de Buchberger e então calcular a redução de $f-g$ sobre essa Base de Gröbner. A saída do Algoritmo da Divisão será zero se e somente se a classe de equivalência de $f$ for igual à de $g$ em $K\left[x_{1}, \ldots, x_{n}\right] / I$. Alternativamente, pode-se calcular a redução de $f$ e de $g$ sobre essa Base de Gröbner. Eles estarão na mesma classe de equivalência se e somente se essas reduções forem iguais.

Vale mencionar ainda que o Algoritmo de Buchberger está descrito de uma maneira vaga, permitindo uma série de implementações alternativas. De fato, existem muitas "melhorias" que podem ser feitas nesse algoritmo, tornando-o mais eficiente.

Em particular, há critérios que permitem ignorar certos tipos de S-polinômios e ainda assim encontrar uma Base de Gröbner, ou mesmo considerá-los em ordens diferentes e com isso reduzir o número de operações realizadas pelo algoritmo. ${ }^{8}$ A busca por tais melhorias constitui ainda hoje uma área bastante ativa de pesquisa e sem resultados conclusivos. O leitor interessado encontra

\footnotetext{
${ }^{8} \mathrm{~A}$ estratégia que uma implementação do Algoritmo de Buchberger utiliza para decidir qual a ordem em que os S-polinòmios são considerado é conhecida na literatura como estratégia de seleção.
} 
muitos artigos na área [Buc79, GMN ${ }^{+} 91$, GM88, Tra89]. Ainda, as Seções 2.9 de [CLO97] e 5.5 de [BW93] fornecem boas introduções a esses assuntos.

Contudo, cabe mencionar que o algoritmo devolve a saída esperada em um número finito de passos para qualquer ordem em que os S-polinômios são considerados e qualquer ordem de reduções realizada sobre eles.

Comentamos também que foram realizados estudos envolvendo a complexidade de tempo e espaço desse algoritmo para diferentes alternativas de implementação. Não exploraremos os detalhes dessas análises, mas cabe mencionar que o Algoritmo de Buchberger consome espaço exponencial. ${ }^{9}$ O leitor interessado encontra maiores detalhes nas Seções 2.9 de [CLO97], 21.7 de [vzGG99] e no apêndice de [BW93]. Um bom resumo dos resultados mais recentes nessa área é dado por E. W. Mayr [May97].

Encerramos a seção citando brevemente algumas áreas de aplicação das Bases de Gröbner comutativas. A literatura é muito rica nesse assunto, de modo que apenas tocaremos nos pontos principais. Conforme visto anteriormente, a Teoria de Bases de Gröbner nos fornece uma solução algorítmica para os problemas da pertinência em ideais e da igualdade em quocientes de $K\left[x_{1}, \ldots, x_{n}\right]$. Ademais, elas possuem sérias aplicações em Geometria Algébrica. Elas fornecem soluções para sistemas de equações polinomiais, a possibilidade de responder (algoritmicamente) perguntas importantes sobre ideais e foram utilizadas em métodos de criptanálise recentemente [FJ03] (graças a sua capacidade de resolver sistemas de equações polinomiais).

O leitor interessado encontra muitos detalhes dessas aplicações (e de várias outras) nos livros sobre o assunto [CLO97, BW93, vzGG99, AL94].

\footnotetext{
${ }^{9}$ É válido comentar que existem diferentes alternativas para medir o "tamanho" de um conjunto de geradores de um ideal de $K\left[x_{1}, \ldots, x_{n}\right]$. Em particular, costuma-se levar em consideração o número de elementos desse conjunto, os graus das variáveis e o tamanho do suporte desses elementos, bem como seus coeficientes. Contudo, é sabido que o Algoritmo de Buchberger pode retornar um conjunto com número de elementos exponencial no número de elementos do conjunto fornecido como entrada [May97].
} 


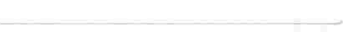




\section{INTRODUÇÃO A BASES DE GRÖBNER NÃO COMUTATIVAS}

Este capítulo fornece uma introdução à Teoria de Bases de Gröbner não comutativas. Ao contrário do que fizemos no Capítulo 2, a apresentação dada aqui será bastante detalhada e extensa, uma vez que esta teoria será o foco de todo o trabalho. Neste capítulo e nos que seguem, abordaremos sempre o caso não comutativo, a menos que explicitado de forma contrária.

As idéias envolvidas na Teoria de Bases de Gröbner não comutativas foram inicialmente estudadas de forma independente por Bokut [Bok76] e por Bergman [Ber78]. De fato, o Lema do Diamante de Bergman tem um forte impacto nesta teoria, como será explicitado na Seção 4.2.6.

Entretanto, quem primeiro considerou essa teoria de um ponto de vista mais computacional foi Mora [Mor86, Mor94], introduzindo uma generalização do Algoritmo de Buchberger para o caso não comutativo (em particular para a álgebra livre), conhecido como Procedimento de Mora.

Desde o surgimento da Teoria de Bases de Gröbner não comutativas, uma série de estudos foram feitos a seu respeito [Mor94, Gre97, Ufn98]. Além disso, outros trabalhos, contendo generalizações, aplicações e refinamentos foram apresentados.

Um desses desenvolvimentos será especialmente interessante para este trabalho. Trata-se da generalização da teoria do contexto das álgebras livres para o das álgebras de caminhos feita por Farkas, Feustel e Green [FFG93, Gre97]. De fato, nossa exposição trabalhará nesse contexto mais geral e não no das álgebras livres.

A seção 5 de [Gre97] traz mais comentários sobre aplicações da teoria de Bases de Gröbner no estudo de módulos, de resoluções projetivas, álgebras de Koszul, entre outras. Vale ressaltar que o uso da Teoria de Bases de Gröbner no estudo de séries de Hilbert e de Poincaré foi explorado por Ufnarovski [Ufn80, Ufn89].

É bem conhecido o fato de que existe uma forte ligação entre a Teoria de Bases de Gröbner e Sistemas de Reescrita de Termos [DJ90]. Apesar de não termos a pretensão de explorar nenhum aspecto dessa ligação neste trabalho, comentamos que a computação de uma Base de Gröbner pode ser vista como uma forma especial do método de Knuth-Bendix. Um polinômio pode ser considerado como uma regra de reescrita e, com isso, uma Base de Gröbner passa a ser um conjunto convergente de regras de reescrita. Bündgen [Bün91, Bün92] mostra que de fato o Algoritmo de Buchberger corresponde ao método de Knuth-Bendix.

Por outro lado, podemos considerar sistemas de reescrita de strings [BO93] (eles são uma forma particular de sistemas de reescrita de termos). Assim, o método de Knuth-Bendix para esses sistemas particulares de reescrita pode ser visto como um caso particular do Procedimento de Mora. Tal 
ligação foi explorada por Heyworth [Hey00].

É importante ressaltar que existem uma série de pacotes computacionais que implementam os algoritmos e procedimentos da Teoria de Bases de Gröbner não comutativas. Entre eles, destacamos:

Opal O pacote Opal foi desenvolvido por B. Keller como parte de sua tese de doutorado [Kel97, Kel98, GHK97]. Ele trabalha no contexto das álgebras de caminhos.

NCGB O pacote NCGB foi desenvolvido por M. Stankus, J. W. Helton et al. e trabalha no contexto das álgebras livres. Ele foi desenvolvido para ser utilizado em conjunto com o software Mathematica.

Bergman Desenvolvido por J. Backelin, V. Ufnarovski et al. em Standard Lisp. O pacote Bergman trabalha com ideais homogêneos no contexto da álgebra livre.

GNBP Consiste de um pacote para o GAP4 implementado por A. M. Cohen e D. A. H. Gijsbers. Trabalha com a álgebra livre.

Deve estar claro que abordaremos neste capítulo e no seguinte uma generalização do que foi apresentado de forma sucinta no capítulo anterior sobre Bases de Gröbner comutativas. Dessa forma, o leitor pode ter em mente o problema da pertinência para ideais abordado durante todo o Capítulo 2.

Existem muitas semelhanças entre o que será feito daqui em diante e a linha do que foi feito nas Seções 2.2, 2.3 e 2.4: utilizaremos um conjunto gerador "especial" de um ideal para "dividir" um elemento do ideal por esse conjunto. A pertinência do elemento no ideal pode então ser decidida analisando-se o "resto" dessa divisão.

Contudo, vale mencionar que nessa generalização surgem alguns problemas inexistentes no caso comutativo. Os seguintes problemas serão discutidos detalhadamente em suas respectivas seções, mas é válido apresentar um breve resumo neste momento:

- No caso comutativo há sempre a garantia de existência de uma Base de Gröbner finita, de forma que o Algoritmo de Buchberger sempre termina sua computação (Teorema 2.4.6). Para o caso não comutativo, entretanto, existem exemplos de ideais finitamente gerados que não possuem nenhuma Base de Gröbner finita, independente da ordem dada nos termos (vide Exemplo 3.2.3).

Com isso, temos que o procedimento de Mora pode nunca finalizar sua computação. Por outro lado, existe a garantia de que se o ideal admitir alguma Base de Gröbner finita (para a ordem dada) então o procedimento de Mora terminará e devolverá uma Base de Gröbner finita.

- O problema de calcular uma Base de Gröbner não é computável. ${ }^{1}$ A demonstração original para esse fato é atribuída a Mora [Mor91, Mor94] e foi incluída na Seção 4.4.

Essa demonstração consiste em reduzir o problema da palavra (sabidamente indecidível) para o problema da pertinência para ideais. Então, se uma Base de Gröbner pode ser construída, podemos resolver o problema de pertinência para um dado elemento, por meio do algoritmo da divisão, o que nos levaria a uma solução para o problema da palavra.

\footnotetext{
${ }^{1}$ Adiaremos uma discussão sobre o sentido preciso dessa afirmação para a Seção 4.4.
} 
Outros pontos que destacam a maior complexidade do caso não comutativo quando comparado com a versão comutativa da teoria foram apresentados por Green, Mora e Ufnarovski [GMU98].

Diante desses problemas, surgiram muitos estudos que se restringem a classes de álgebras para as quais os problemas descritos acima se tornam mais tratáveis. Entre elas, mencionamos álgebras solúveis, introduzidas por Kandri-Rody e Weispfenning [KRW90], álgebras de envelopamento de álgebras de Lie, álgebras que sejam o quociente de uma álgebra livre por um ideal homogêneo [Mor94] e álgebras bi-autômato (introduzidas por Mansson e Nordbeck [MN02] e exploradas no Capítulo 5). Outras generalizações da teoria foram apresentadas por Mora [Mor94].

Este capítulo apresenta inicialmente as bases da teoria com resultados e definições iniciais na Seção 3.1. Em seguida, apresentamos na Seção 3.2 o conceito de Base de Gröbner e exploramos suas principais propriedades e exemplos. A Seção 3.3 contém um resultado importante sobre álgebras de dimensão finita que afirma que se uma álgebra $R / I$ tem dimensão finita, então o ideal $I$ possui uma Base de Gröbner finita. Ainda nessa seção, apresentaremos mais dois resultados envolvendo álgebras com essa propriedade.

Ainda, a Seção 3.4 apresenta propriedades fundamentais das álgebras para as quais se pode desenvolver uma Teoria de Bases de Gröbner não comutativas e estabelece um forte paralelo entre estas e as álgebras de caminhos. Em particular, apresentaremos um teorema de Green [Gre00] que afirma que toda álgebra para a qual se pode desenvolver uma Teoria de Bases de Gröbner não comutativas é um quociente de uma álgebra de caminhos.

Finalmente, o Algoritmo da Divisão, o Procedimento de Mora e algumas questões envolvendo computabilidade (incluindo as questões acima mencionadas) serão abordados no Capítulo 4.

\subsection{Conceitos Iniciais}

Daqui em diante, denotaremos por $K$ um corpo e trabalharemos com uma álgebra $R$ sobre $K$. Além disso, assumiremos sempre que nossas álgebras são finitamente geradas.

Assumiremos ainda que $\mathcal{B}$ é uma base de $R$ como espaço vetorial multiplicativa, isto é, para todos $b_{1}$ e $b_{2}$ em $\mathcal{B}$, vale

$$
b_{1} \cdot b_{2} \in \mathcal{B} \cup\{0\}
$$

Em outras palavras, $\mathcal{B}$ é uma base multiplicativa se $\mathcal{B} \cup\{0\}$ for um semigrupo.

Vale comentar que a noção dada aqui de base multiplicativa pode ser vista como uma generalização do conceito de base multiplicativa utilizado em outras áreas da Teoria de Representações de Álgebras [BGRS85]. Entretanto, os conceitos se tornam muito parecidos a partir do momento em que fixamos uma ordem admissível nesta base, conforme será feito adiante.

Chamaremos os elementos de uma tal base $\mathcal{B}$ de monômios ou termos. Por hora, assumiremos como hipótese que a divisão em $\mathcal{B}$ é reflexiva, no seguinte sentido: se $b$ é um elemento de $\mathcal{B}$, então existem $b_{1}$ e $b_{2}$ em $\mathcal{B}$ tais que $b=b_{1} b b_{2}$. Uma conseqüência importante dessa suposição é que um monômio $b$ divide outro monômio $a$ se e somente se existem monômios $b_{1}$ e $b_{2}$ tais que $a=b_{1} b b_{2}$. Mais adiante, o Corolário 3.4.3 nos mostrará que essa hipótese não constitui nenhuma perda de generalidade, uma vez que todas as bases multiplicativas que servem para os nossos propósitos possuem essa propriedade.

Dado um alfabeto finito $X=\left\{x_{1}, x_{2}, \ldots, x_{a}\right\}$, podemos mencionar como exemplo a álgebra livre sobre $X$, denotada por $K\langle X\rangle$. Neste caso, o conjunto $X^{*}$ das palavras sobre $X$ forma uma base 
multiplicativa de $K\langle X\rangle$.

Ainda, dado um grafo (finito) $\Gamma$, podemos considerar a álgebra de caminhos $K \Gamma$, com o conjunto de todos os caminhos em $\Gamma$ (denotado por $\mathcal{P}$ ) constituindo uma base multiplicativa para $K \Gamma$. Esses de fato, são os os dois exemplos centrais que utilizaremos ao longo de todo o trabalho.

Por um lado, a álgebra livre pode ser vista como um caso especial das álgebras de caminhos, conforme comentado na Seção 1.4. Entretanto, optamos por explicitar exemplos e particularidades desse caso devido ao seu largo uso e por ele ter sido o contexto do trabalho original de Mora.

Prosseguimos então apresentando o conceito de ordem admissível. Esse conceito é uma generalização da definição de ordem monomial vista para o caso comutativo (Definição 2.4.1) e tem exatamente o mesmo objetivo.

Definição 3.1.1 (Ordem Admissivel) Dizemos que uma ordem $\leq$ em uma base multiplicativa $\mathcal{B}$ é admissível se, para todos $p, q, r$ em $\mathcal{B}$, as seguintes condições forem satisfeitas.

1. $\leq$ é uma boa ordem em $\mathcal{B}$.

2. $p<q$ implica $r p<r q$, se ambas as multiplicações forem não nulas.

3. $p<q$ implica $p r<q r$, se ambas as multiplicações forem não nulas.

4. $p$ divide $q$ implica $p \leq q$.

Seguem alguns exemplos de ordens admissíveis para a álgebra livre e para as álgebras de caminhos. Considere, para tanto, as bases multiplicativas $X^{*}$ de $K\langle X\rangle$ e $\mathcal{P}$ de $K \Gamma$.

Ordem de grau e lexicografia (abreviada por deglex) A ordem de grau e lexicografia é provavelmente a ordem admissível mais utilizada ao se lidar com Bases de Gröbner não comutativas. Contudo, antes de fornecer sua definição, é preciso mostrar como ordenar elementos lexicograficamente.

Para a álgebra livre, podemos dar uma ordem total nas letras de $X$. Com isso, dizemos que $u=x_{i_{1}} x_{i_{2}} \cdots x_{i_{k}}$ em $X^{*}$ é lexicograficamente menor que outra palavra $w=x_{j_{1}} x_{j_{2}} \cdots x_{j_{l}}$ se

$w=u k$, para algum $1 \neq k \in X^{*}$ ou

existe $1 \leq h \leq \min (k, l)$ tal que $x_{i_{t}}=x_{j_{t}}$, para $1 \leq t<h$ e $x_{i_{h}}<x_{j_{h}}$, de acordo com a ordem dada nos elementos de $X$.

Vale observar que esta é exatamente a ordem alfabética utilizada em dicionários (o que justifica seu nome). Para ver alguns exemplos, podemos assumir que $X=\{a, b, c\}$ e que a ordem dada nos elementos de $X$ é $a<b<c$. Então teremos:

$$
\begin{aligned}
& 1<a^{2}<b<b^{2} \\
& a b^{2}<a b^{3}<b \\
& a b>a^{2} b>a^{3} b>a^{4} b>\cdots
\end{aligned}
$$


Note o leitor que a ordem lexicográfica definida acima é uma ordem total. Ainda, vale salientar que essa ordem, embora utilizada para o caso comutativo como monomial não é admissível. De fato, o terceiro exemplo acima nos mostra uma cadeia descendente infinita em $K\langle a, b, c\rangle$. Isso mostra que a ordem lexicográfica não é uma boa ordem em $X^{*}$ e, portanto, não é admissível para o caso das álgebras livres (e, conseqüentemente, não é admissível para o caso mais geral das álgebras de caminhos, visto a seguir).

A ordem lexicográfica em $\mathcal{P}$ para o caso das álgebras de caminhos é definida de maneira bastante análoga. Dada uma álgebra de caminhos $K \Gamma$, ordenamos totalmente e independentemente os vértices e os arcos de $\Gamma$ (como estamos assumindo que nossas álgebras são finitamente geradas, esses dois conjuntos são finitos). Com isso, dados dois caminhos $p=p_{1} p_{2} \cdots p_{k} \mathrm{e}$ $q=q_{1} q_{2} \cdots q_{l}$ escritos na forma canônica, temos que $p$ é lexicograficamente menor que $q$ se

$p$ é um vértice e $q$ é um caminho de comprimento não nulo ou $p$ e $q$ são vértices e $p<q$ na ordem definida entre os vértices ou $p$ e $q$ são caminhos de comprimento não nulo e

$q=p k$, para algum caminho $k$ de comprimento não nulo ou existe $1 \leq h \leq \min (k, l)$ tal que $p_{t}=q_{t}$, para $1 \leq t<h$ e $p_{h}<q_{h}$, de acordo com a ordem dada nos arcos.

Seguem alguns exemplos para a álgebra de caminhos construída a partir do grafo $\Gamma$ da figura abaixo. Considere $v_{1}<v_{2}<\cdots<v_{5}$ e $a<b<c<d<e<f$.

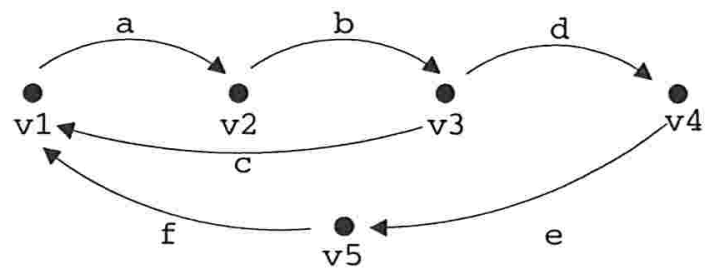

$$
\begin{aligned}
& v 1<a<a b<b<d e f \\
& a b c<d e<e \\
& d>c a b d>(c a b)^{2} d>\ldots
\end{aligned}
$$

Chamamos a atenção para o fato de que a ordem lexicográfica definida acima para álgebras de caminhos é compativel com a definida para a álgebra livre, no seguinte sentido. Se tomamos $X=\left\{x_{1}, x_{2}, \ldots, x_{a}\right\}$ e a álgebra livre $K\langle X\rangle$, podemos construir um grafo $H$ com apenas um vértice e $a$ laços e teremos um isomorfismo $\varphi: K\left\langle x_{1}, x_{2}, \ldots, x_{a}\right\rangle \longrightarrow K H{ }^{2}$ Seja ainda $<_{1}$ a ordem lexicográfica definida para a álgebra livre e $<_{2}$ a definida para álgebras de caminhos. Então, se $b, c$ pertencem a $X^{*}, b<_{1} c$ se e somente se $\varphi(b)<_{2} \varphi(c)$.

Finalmente podemos dizer como ordenar elementos utilizando a ordem de grau e lexicografia. Sejam $p, q$ dois caminhos em $\mathcal{P}$ (ou duas palavras de $X^{*}$ ). Então $p$ é menor que $q$ se

\footnotetext{
${ }^{2}$ Detalhes desta construção são dados na Seção 1.4
} 


$$
\begin{aligned}
& \|p\|<\|q\| \text { ou } \\
& \|p\|=\|q\| \text { e } p \text { é menor que } q \text { lexicograficamente. }
\end{aligned}
$$

Seguem alguns exemplos para $K\langle a, b, c\rangle(\operatorname{com} a<b<c)$ :

$$
\begin{aligned}
& 1<b<a^{2}<b^{2}<a^{3} \\
& b a<b^{2} a<b^{3} a<b^{4} a
\end{aligned}
$$

Para ver alguns exemplos para a álgebra de caminhos, considere o grafo $\Gamma$ visto anteriormente, com a mesma ordem dada nos vértices e arcos.

$$
\begin{aligned}
& v 1<a<b<a b<d e f \\
& e<d e<c a b<c a b d<(c a b)^{2} d
\end{aligned}
$$

Deixamos para o leitor os detalhes da verificação de que a ordem de grau e lexicografia é admissível. Para ver que ela é uma boa ordem, basta notar que o conjunto formado pelos graus de todos os elementos de $\mathcal{P}$ (ou de $X^{*}$ ) é bem ordenado (é um subconjunto de $\{0,1,2,3, \ldots\}$ ). Além disso, uma vez que tanto o grafo, quanto o alfabeto considerados são finitos, existe apenas um número finito de elementos com o mesmo grau.

Então, se tomamos um subconjunto $A$ de $\mathcal{P}$ (ou de $X^{*}$ ), existe um inteiro $i \geq 0$, que é o mínimo dentre os graus dos elementos de $A$. Assim, podemos tomar $B$ como sendo o subconjunto de $A$ composto pelos elementos de grau igual a $i$. Não é difícil ver que todos os elementos de $A \backslash B$ são estritamente maiores que os elementos de $B$ (pela definição da ordem de grau e lexicografia) e que $B$ é finito. Portanto, $B$ é um conjunto finito e totalmente ordenado, donde segue que $B$ possui um menor elemento $b$, que será o menor elemento de $A$.

Ordem de peso e lexicografia (abreviada por weight-lex) Esta ordem constitui uma generalização da ordem deglex e é definida para $K\langle X\rangle$ da seguinte forma. Dada uma aplicação peso $\phi: X \longrightarrow\{1,2,3, \ldots\}$, definimos $\phi: X^{*} \longrightarrow\{0,1,2,3, \ldots\}$ sua extensão natural. Ou seja, se $a=x_{i_{1}} x_{i_{2}} \cdots x_{i_{k}}$ é um elemento de $X^{*}$, então $\phi(a)=\sum_{j=1}^{k} \phi\left(x_{i_{j}}\right)$ (com a convenção de que $\phi(1)=0$ ). Com isso, dadas duas palavras $u$ e $w$, dizemos que $u$ é menor que $w$ se

$$
\begin{aligned}
& \phi(u)<\phi(w) \text { ou } \\
& \phi(u)=\phi(w) \text { e } u \text { é menor que } w \text { lexicograficamente }
\end{aligned}
$$

A mesma ordem pode ser generalizada para uma álgebra de caminhos $K \Gamma$ se tivermos uma aplicação $\phi: A(\Gamma) \longrightarrow\{1,2,3, \ldots\}$ e a estendermos para $\phi: \mathcal{P} \longrightarrow\{0,1,2,3, \ldots\}$ da seguinte maneira. Seja $p=e_{1} e_{2} \cdots e_{k}$ um caminho escrito em sua forma canônica. Então $\phi(p)=0$ se $p$ é um vértice ou $\phi(p)=\sum_{i=1}^{k} \phi\left(e_{i}\right)$.

Ordem de peso e lexicografia reversa (abreviada por rev-weight-lex) Esta ordem é muito similar à anterior, diferindo apenas no fato de que ela utiliza a ordem de lexicografia reversa para ordenar elementos de mesmo peso (valor de $\phi$ ). Resta-nos então definir a ordem lexicográfica reversa para $X^{*}$ e para $\mathcal{P}$.

Dada uma ordem total nas letras de $X$ e se $u=x_{i_{1}} x_{i_{2}} \cdots x_{i_{k}}$ e $w=x_{j_{1}} x_{j_{2}} \cdots x_{j_{t}}$ são duas palavras de $X^{*}$, dizemos que $u<w$ se 
$w=a u$, para algum $1 \neq a \in X^{*}$ ou

existe $0 \leq h<\min (k, l)$ tal que $x_{i_{k-t}}=x_{j_{l-t}}$, para $0 \leq t<h$ e $x_{i_{k-h}}<x_{j_{l-h}}$, de acordo com a ordem dada nos elementos de $X$.

De maneira similar, podemos definir a ordem lexicográfica reversa para $\mathcal{P}$. Como antes, ordene totalmente e independentemente os vértices e os arcos de $\Gamma$. Então, dados dois caminhos $p=p_{1} p_{2} \cdots p_{k}$ e $q=q_{1} q_{2} \cdots q_{l}$ escritos na forma canônica, temos que $p<q$ se

$p$ é um vértice e $q$ é um caminho de comprimento não nulo ou

$p$ e $q$ são vértices e $p<q$ na ordem definida entre os vértices ou

$p$ e $q$ são caminhos de comprimento não nulo e

$q=a p$, para algum caminho $a$ de comprimento não nulo ou

existe $0 \leq h<\min (k, l)$ tal que $p_{k-t}=q_{l-t}$, para $0 \leq t<h$ e $p_{k-h}<q_{l-h}$,

de acordo com a ordem dada nos arcos.

Ordem comutativo-lexicográfica (abreviada por comut-lex) Considere $[X]$ o monóide livre comutativo sobre o alfabeto $X$. Seja $\varphi: X^{*} \longrightarrow[X]$ o homomorfismo canônico. Note que $[X]$ é precisamente o conjunto dos monômios do anel de polinômios comutativo em $a$ variáveis. Então $u \in X^{*}$ é menor que $w \in X^{*}$ se

$\varphi(u)<\varphi(w)$ utilizando a ordem deglex definida para o caso comutativo (vide Seção 2.4) ou

$\varphi(u)=\varphi(w)$ e $u$ é menor que $w$ lexicograficamente.

Antes de prosseguirmos com alguns resultados fundamentais envolvendo ordens admissíveis, faremos uma breve discussão sobre alguns aspectos da própria definição desse conceito. Em particular, as condições 2 , 3 e 4 da definição são comumente formuladas de maneira distinta à aqui apresentada quando a definição é referente apenas à álgebra livre (e essa é uma situação comum).

Em linhas gerais, ao trabalhar com a álgebra livre, podemos agrupar as condições 2 e 3 em apenas uma e eliminar a condição 4. Formalizamos essa discussão nas seguintes afirmações.

Afirmação 3.1.1 Seja $\mathcal{B}$ uma base multiplicativa tal que $\mathcal{B} \cdot \mathcal{B} \subseteq \mathcal{B}$ (i.e. todas as multiplicações envolvendo elementos de $\mathcal{B}$ são não nulas) e sejam $p, q \in \mathcal{B}$. Então as condições 2 e 3 da Definição 3.1.1 são equivalentes a

2'. $p<q$ implica upr $<$ uqr, para todos $u, r$ em $\mathcal{B}$

Demonstração. Mostraremos inicialmente que se as condições 2 e 3 são verdadeiras, então a condição 2' também é verdadeira (nas hipóteses da afirmação). Sejam então $p, q \in \mathcal{B}$ com $p<q$. Como as multiplicações entre elementos da base são não nulas, concluímos que, dado $u$ um elemento qualquer de $\mathcal{B}, u p<u q$. Novamente, como as multiplicações entre elementos de $\mathcal{B}$ são não nulas, dado outro elemento qualquer $r$ em $\mathcal{B}$, temos que $u p r<u q r$, o que encerra essa parte da demonstração.

Para o outro lado, assuma que 2' não implica as condições 2 e 3 . Então existem $p, q \in \mathcal{B}$, tais que $p<q$ e $u p \geq u q$ ou $p u \geq q u$. Mas se $u p \geq u q$ e todas as multiplicações entre 
elementos de $\mathcal{B}$ são não nulas, podemos escolher elementos $u^{\prime}$ e $r$ de $\mathcal{B}$ e considerar os elementos $u^{\prime} u p r$ e $u^{\prime} u q r$ (ambos não nulos por hipótese). Então teremos dois casos: se $u p>u q$, então pela condição 2' temos que $u^{\prime} u p r>u^{\prime} u q r$; se $u p=u q$, claramente $u^{\prime} u p r=u^{\prime} u q r$. Logo podemos concluir que $u^{\prime} u p r \geq u^{\prime} u q r$. Mas isso é uma contradição, pois, pela condição 2', $p<q$ (nossa hipótese inicial) implica $u^{\prime} u p r<u^{\prime} u q r$; logo $u p<u q$. Analogamente, teremos que $p u<q u$, o que encerra a demonstração.

Afirmação 3.1.2 Seja $\mathcal{B}$ uma base multiplicativa de uma álgebra $R$ tal que 1 (a unidade de $R$ ) pertence a $\mathcal{B}$. Então, a condição 4 da Definição 3.1 .1 é equivalente a

\section{4'. $1 \leq p$, para todo $p$ em $\mathcal{B}$}

Demonstração. O fato de que 4 implica 4' é imediato, uma vez que 1 divide todo elemento de $\mathcal{B}$.

Para a implicação reversa, tome $p$ e $q$ em $\mathcal{B}$, tais que $p$ divide $q$. Isso ocorre se e somente se existem $u$ e $v$ em $\mathcal{B}(u, v$ ou ambos podem ser iguais a 1$)$ tais que $q=u p v$. Mas então

$$
1 \leq u \Rightarrow 1 \cdot p v \leq u p v=q
$$

Por outro lado,

$$
1 \leq v \Rightarrow p 1 \leq p v \leq q
$$

e $p \leq q$, como queríamos.

Afirmação 3.1.3 Seja $\mathcal{B}$ uma base multiplicativa de uma álgebra $R$ tal que 1 (a unidade de $R$ ) pertence a $\mathcal{B}$ e $\mathcal{B} \cdot \mathcal{B} \subseteq \mathcal{B}$ (i.e todas as multiplicações envolvendo elementos de $\mathcal{B}$ são não nulas). Então as condições 1, 2 e 3 da Definição 3.1 .1 implicam $1 \leq b$, para todo b em $\mathcal{B}$.

Demonstração. Tome uma ordem $\leq$ em $\mathcal{B}$ satisfazendo as condições 1,2 e 3 da Definição 3.1.1. Mostraremos que $1 \leq b$, para todo $b$ em $\mathcal{B}$.

Suponha que a afirmação é falsa e seja $b \in \mathcal{B}$ um contra exemplo, isto é $1>b$. Como as multiplicações em $\mathcal{B}$ são não nulas, $b^{i} \neq 0$, para todo $i>0$.

Assim, pela compatibilidade da ordem $\leq$ com a multiplicação em $\mathcal{B}$, teremos:

$$
1>b \Rightarrow b>b^{2} \Rightarrow b^{2}>b^{3} \Rightarrow \cdots
$$

Isso nos leva a construir a seguinte cadeia descendente infinita: $1>b>b^{2}>\cdots$, contradizendo a hipótese de $\leq$ ser uma boa ordem (vide Proposição 1.1.1).

Com isso, diante das hipóteses de que as multiplicações em $\mathcal{B}$ são não nulas (i.e. $\mathcal{B} \cdot \mathcal{B} \subseteq \mathcal{B}$ ) e de que 1 pertence a $\mathcal{B}$, podemos dizer simplesmente que uma ordem admissível é uma boa ordem que satisfaz:

Se $u \leq v$, então $a u b \leq a v b$, para todos $u, v, a$ e $b$ em $\mathcal{B}$. 
Conforme mencionado anteriormente, essa definição de ordem admissível é muitas vezes utilizada em abordagens da teoria no contexto das álgebras livres.

Chamamos a atenção do leitor para duas propriedades bastante importantes de ordens admissíveis. Primeiro, uma vez que toda ordem admissível é por definição uma boa ordem, elas gozam da propriedade de que toda cadeia descendente infinita de elementos estabiliza (Proposição 1.1.1). Esse fato foi utilizado na demonstração da afirmação acima. Além disso, toda cadeia descendente de divisão estabiliza, como nos mostra a seguinte proposição.

Proposição 3.1.4 Seja $\mathcal{B}$ uma base multiplicativa de uma álgebra $R$ e seja $\leq$ uma ordem admissível em $\mathcal{B}$. Então não existem seqüências infinitas de elementos distintos de $\mathcal{B}$ da forma $b_{1}, b_{2}, b_{3}, \ldots$ tal que $b_{i}$ divide $b_{i-1}$, para $i \geq 2$.

Demonstração. Para provar a proposição, suponha por absurdo que ela seja falsa. Então existe uma cadeia infinita de elementos da forma descrita no enunciado da proposição. Mas, pela definição de ordem admissível (propriedade 4), temos que $b_{1}>b_{2}>b_{3}>\cdots$ é uma cadeia descendente infinita, o que é um absurdo, dado que $\leq$ é uma boa ordem.

Outra observação importante é a de que se uma base multiplicativa $\mathcal{B}$ admite alguma ordem admissível, então a divisão em $\mathcal{B}$ é uma relação antissimétrica. Claro que esta propriedade não é válida para bases multiplicativas em geral, uma vez que uma base multiplicativa é simplesmente um semigrupo com zero.

Lema 3.1.5 Seja $\mathcal{B}$ uma base multiplicativa que admite alguma ordem admissivel. Se dois elementos $b_{1}$ e $b_{2}$ de $\mathcal{B}$ são tais que $b_{1}$ divide $b_{2}$ e $b_{2}$ divide $b_{1}$, então $b_{1}=b_{2}$.

Demonstração. Tome $\leq$ uma ordem admissível em $\mathcal{B}$. Então, pela definição de ordem admissível, as hipóteses do lema implicam $b_{1} \leq b_{2}$ e $b_{2} \leq b_{1}$.

Com isso, se $\mathcal{B}$ é uma base multiplicativa munida de uma ordem admissível, podemos considerar a divisão em $\mathcal{B}$ como uma ordem parcial. Essa propriedade da divisão será usada livremente no restante do texto.

Assumiremos daqui por diante que temos sempre uma base multiplicativa $\mathcal{B}$ em $R$ com uma ordem admissível fixada. Em geral, dada uma álgebra $R$, diremos que $R$ admite uma teoria de Bases de Gröbner se existem uma base multiplicativa $\mathcal{B}$ de $R$ e uma ordem admissível $\leq$ em $\mathcal{B}$.

Lma vez que temos uma ordem admissivel em $\mathcal{B}$, podemos, dado um elemento não nulo $f$ de $R$, encontrar o maior elemento de seu suporte (o suporte de $f$ é um conjunto finito, não vazio e totalmente ordenado pela ordem admissível dada). Esse é precisamente o conceito de termo líder.

Definição 3.1.2 (Termo Lider) Seja $R$ uma álgebra com uma base multiplicativa $\mathcal{B}$ e $\leq$ uma ordem admissivel em $\mathcal{B}$. Entăo, se $f$ é um elemento não nulo de $R$, definimos como seu termo líder o maior elemento do suporte de $f$ (de acordo com a ordem admissivel $\leq$ ) e o denotamos por $\hat{f}$. Chamaremos ainda o coeficiente de $\widehat{f}$ de coeficiente lider de $f$ (denotado por cl $(f)$ ). Ademais, se $A$ é um subconjunto de $R$, definimos $\widehat{A}:=\{\widehat{a} \mid a \in A\}$. 
Conforme veremos mais adiante (na realidade, o leitor pode recordar isso de nossa abordagem da Teoria de Bases de Gröbner comutativas), será muito importante trabalharmos com o conjunto dos termos líderes de um ideal $I$ de $R$. Por outro lado, também merecem destaque os elementos de $\mathcal{B}$ que não são termos líderes de nenhum elemento desse ideal $I$. Para lidarmos com esses elementos, apresentamos a seguinte definição.

Definição 3.1.3 (Termo Normal) Dado um subconjunto $A$ de $R$, um elemento $b$ de $\mathcal{B}$ é dito normal com respeito a $A$ se não existe $h$ em $A$ tal que $\widehat{h}=b$. Denotaremos ainda por Norm(A) o conjunto dos termos normais com respeito a $A$, ou seja $\operatorname{Norm}(\mathrm{A})=\mathcal{B} \backslash \widehat{A}$.

Vale a observação simples de que, com a notação da Definição 3.1.3, se $A$ é um ideal, podemos substituir a condição " $\widehat{h}=b$ " por " $\widehat{h}$ divide $b$ ". Também deve ficar claro para o leitor que, neste caso em que $A$ é um ideal da álgebra $R, \widehat{A} \cup\{0\}$ é um ideal de semigrupo de $\mathcal{B} \cup\{0\}$ e Norm(A) é um ideal de ordem de $\mathcal{B}$, isto é, se $b^{\prime}$ divide $b$ e $b$ é um elemento de Norm(A), então $b^{\prime}$ também pertence a $\operatorname{Norm}(\mathrm{A})$.

No que segue, assumiremos que temos um ideal $I$ de $R$ (dado por um conjunto gerador) e chamaremos de $N$ o espaço vetorial gerado pelos elementos de $\mathcal{B}$ que são normais com respeito a $I$, ou seja $N=\operatorname{span}(\operatorname{Norm}(\mathrm{I}))$. O seguinte teorema possui conseqüências centrais na Teoria de Bases de Gröbner não comutativas.

Teorema 3.1.6 Seja $R$ uma álgebra com uma base multiplicativa $\mathcal{B}$ e $\leq$ uma ordem admissível fixada em $\mathcal{B}$. Seja ainda I um ideal de $R$ e $N=\operatorname{span}(\operatorname{Norm}(\mathrm{I}))$. Então

$$
R=I \oplus N \text { (como espaço vetorial) }
$$

Demonstração. Para provar o resultado precisamos mostrar que a intersecção de $I$ com $N$ é $\{0\}$ e que todo elemento $f$ de $R$ se escreve como $f=f_{I}+N(f)$, onde $f_{I} \in I$ e $N(f) \in N$.

Para verificar que a intersecção é zero, assuma $g$ um elemento não nulo em $I \cap N$. Então, como $g$ é um elemento de $R$, ele se escreve como combinação linear dos elementos de $\mathcal{B}$ e, como ele é não nulo, seu suporte não é vazio. Logo, podemos encontrar o maior elemento do suporte de $g, \widehat{g}$. Portanto, como $g$ pertence a $I$, segue, por definição, que $\widehat{g} \in \widehat{I}$. Por outro lado, como $g$ é um elemento de $N=\operatorname{span}(\operatorname{Norm}(\mathrm{I}))$ e $\widehat{g}$ é um elemento do suporte de $g$, segue que $\widehat{g} \in N$, uma contradição com a definição de $N$. Logo, concluímos que $I \cap N=\{0\}$.

Para a segunda parte, usaremos a técnica de indução noetheriana, comentada na Seção 1.1. Suponha que o resultado é falso e seja $f$ não nulo um contra exemplo de termo líder mínimo, isto é, $f$ não se escreve como $f=f_{I}+N(f)$, mas todo elemento não nulo com termo líder menor que $\hat{f}$ se escreve dessa forma.

Então temos duas possibilidades: $\widehat{f}$ pertence a $\widehat{I}$ ou $\widehat{f}$ pertence a $N$. No primeiro caso, podemos tomar $s$ em $I$ tal que $\widehat{s}=\widehat{f}$. Mas então, $f^{\prime}:=f-(\operatorname{cl}(f) / \operatorname{cl}(s)) s$ possui termo líder menor que $\widehat{f}$ ou é zero. No primeiro caso, segue, pela minimalidade de $\widehat{f}$, que $f^{\prime}$ se escreve como $f^{\prime}=f_{I}^{\prime}+N\left(f^{\prime}\right)$, com $f_{I}^{\prime}$ em $I$ e $N\left(f^{\prime}\right)$ em $N$. Portanto, $f=$ $f_{I}^{\prime}+(\operatorname{cl}(f) / \operatorname{cl}(s)) s+N\left(f^{\prime}\right) \in I+N$, uma contradição. No segundo caso, $(\operatorname{cl}(f) / \operatorname{cl}(s)) s=$ $f \in I \subseteq I+N$, nos levando a uma contradição. 
Por outro lado, se $\widehat{f} \in N$, podemos tomar $f^{\prime}:=f-c l(f) \widehat{f}$. Analogamente, $f^{\prime}=$ $f_{I}^{\prime}+N\left(f^{\prime}\right), \operatorname{com} f_{I}^{\prime}$ em $I$ e $N\left(f^{\prime}\right)$ em $N$ ou $f^{\prime}=0$. Assim, $f=f_{I}^{\prime}+N\left(f^{\prime}\right)+c l(f) \widehat{f} \in I+N$, ou $f=c l(f) \widehat{f} \in N \subseteq I+N$, o que encerra a demonstração.

Uma vez que o teorema acima possui conseqüências fundamentais para o restante da teoria, é válido explorar melhor seu significado. Uma das grandes conseqüências desse teorema é que, dado um ideal $I$ de $R$, podemos escrever um elemento $f$ de $R$ de forma única como $f=f_{I}+N(f)$ (com $f_{I}$ em $I$ e $N(f)$ em $\left.N\right)$. Devemos destacar que esse resultado nos dá o respaldo para definir uma função de $R$ em $N$ que associa cada elemento $f$ de $R$ à sua forma normal $N(f)$. Formalizamos esse fato na seguinte definição.

Definição 3.1.4 (Forma Normal) Seja $R$ uma álgebra com uma base multiplicativa $\mathcal{B}$ e $\leq$ uma ordem admissivel fixada em $\mathcal{B}$. Seja ainda $I$ um ideal de $R, N=\operatorname{span}(\operatorname{Norm}(\mathrm{I}))$ e $f$ um elemento de $R$. Logo, pelo Teorema 3.1.6, existe um único $N(f)$ em $N$, tal que $f$ se escreve como $f=f_{I}+N(f)$, com $f_{I}$ em $I$.

Definimos então $N(f)$ como a forma normal de $f$ módulo $I$. Denotaremos esse elemento sempre por $N(f)$ ou por $N_{I}(f)$ quando for necessário explicitar o ideal a que a forma normal se refere.

Um problema que temos considerado como motivador até aqui é o problema da pertinência para ideais. Note que $f$ pertence a $I$ se e somente se $N(f)=0$. Mais que isso, dois elementos de $R$ estão na mesma classe de equivalência de $R / I$ se e somente se suas formas normais forem iguais. Com isso, se há interesse em estudar o quociente $R / I$, as formas normais são bons representantes de classes de elementos de $R / I$ (para, por exemplo, representar essas classes em um computador).

Podemos ainda definir uma estrutura multiplicativa em $N$ da seguinte forma: para multiplicar dois elementos de $N$, basta efetuar a multiplicação utilizando a estrutura de $R$ e tomar a forma normal do resultado, ou seja, dados $f, f^{\prime}$ em $N, f \cdot N f^{\prime}:=N\left(f \cdot_{R} f^{\prime}\right)$. A soma em $N$ pode ser feita da mesma forma que em $R$, dado que $N\left(f+f^{\prime}\right)=N(f)+N\left(f^{\prime}\right)$. Com isso, $N$ (munido dessas operações) é isomorfo como álgebra a $R / I$ e computações em $R / I$ podem ser feitas por meio do cálculo de formas normais.

Detalhamos a discussão acima em uma seqüência de corolários do Teorema 3.1.6. Contudo, antes de deixarmos o leitor a sós com as demonstrações, é importante ressaltar que as formas normais representam os "restos" da divisão especiais mencionados no início do capítulo. De fato, elas são a saída do Algoritmo da Divisão para o caso não comutativo (que será estudado adiante) e têm o mesmo papel que esse resto tinha no caso comutativo. ${ }^{3}$

Se conseguimos calcular formas normais algoritmicamente, então possuímos um algoritmo que decide pertinència e podemos realizar operações em $R / I$. Contudo, veremos que é impossível calcularmos todas as formas normais que gostaríamos no contexto mais geral. O restante deste capítulo e o seguinte concentram seus esforços em desenvolver ferramentas para calcular formas normais.

Para os corolários, assuma $R$ uma álgebra com uma base multiplicativa $\mathcal{B}$ e $\leq$ uma ordem admissivel fixada em $\mathcal{B}$. Seja ainda $I$ um ideal de $R$ e $N=\operatorname{span}(\operatorname{Norm}(\mathrm{I}))$.

\footnotetext{
${ }^{3}$ É importante mencionar que, para que o algoritmo da divisão produza essas formas normais como saída, ele deve ser utilizado em conjunto com uma Base de Gröbner.
} 
Corolário 3.1.7 Seja $f$ em $R$. Então a forma normal de $f$ (módulo $I$ ) é zero se e somente se $f$ pertence a $I$.

Demonstração. Um elemento $f$ pertence a $I$ se e somente se ele se escreve como $f=f+0(\operatorname{com} f$ em $I$ e 0 em $N)$ e, pelo Teorema 3.1.6, isso ocorre se e somente se $N(f)=0$, o que encerra a demonstração.

Corolário 3.1.8 Sejam $f_{1}, f_{2}, \ldots, f_{n}$ elementos de $R$. Então as seguintes afirmações são verdadeiras.

1. Se $f_{1}$ pertence a $N$, então $N\left(f_{1}\right)=f_{1}$.

2. $N\left(N\left(f_{1}\right)\right)=N\left(f_{1}\right)$.

3. $N\left(f_{1}+f_{2}+\cdots+f_{n}\right)=N\left(f_{1}\right)+N\left(f_{2}\right)+\cdots+N\left(f_{n}\right)$.

4. $N\left(f_{1} f_{2} \cdots f_{n}\right)=N\left(N\left(f_{1}\right) \cdot N\left(f_{2}\right) \cdots N\left(f_{n}\right)\right.$ ) (todas as multiplicações são em $R$ ).

5. Se $\lambda$ é um escalar, então $N\left(\lambda f_{1}\right)=\lambda N\left(f_{1}\right)$.

Demonstração. A primeira parte decorre imediatamente da definição de forma normal e do Teorema 3.1.6.

A segunda parte, decorre da primeira parte e do fato de que $N\left(f_{1}\right)$ pertence a $N$.

Para a terceira parte, se escrevermos cada $f_{i}$ como $f_{i}^{I}+N\left(f_{i}\right)$, com $f_{i}^{I}$ em $I$, para $1 \leq i \leq n$, basta notar que:

$$
f_{1}+f_{2}+\cdots+f_{n}=f_{1}^{I}+f_{2}^{I}+\cdots+f_{n}^{I}+N\left(f_{1}\right)+N\left(f_{2}\right)+\cdots+N\left(f_{n}\right)
$$

Para a quarta parte, se escrevermos cada $f_{i}$ como antes, teremos:

$$
f_{1} f_{2} \cdots f_{n}=\left(f_{1}^{I}+N\left(f_{1}\right)\right)\left(f_{2}^{I}+N\left(f_{2}\right)\right) \cdots\left(f_{n}^{I}+N\left(f_{n}\right)\right)
$$

Não é difícil ver que o único elemento que pode não pertencer a $I$ nesse produto é $N\left(f_{1}\right) \cdot N\left(f_{2}\right) \cdots N\left(f_{n}\right)$ e o resultado segue pelo Teorema 3.1.6.

Finalmente, a quinta parte segue do seguinte: $\lambda f_{1}=\lambda f_{1}^{I}+\lambda N\left(f_{1}\right)$; onde $\lambda f_{1}^{I}$ é um elemento de $I$ e $\lambda N\left(f_{1}\right)$ pertence a $N$ e o resultado segue do Teorema 3.1.6.

Corolário 3.1.9 Sejam $f, f^{\prime}$ em $R$. Então $f+I=f^{\prime}+I$ se e somente se $N_{I}(f)=N_{I}\left(f^{\prime}\right)$.

Demonstração. Para tornar a notação mais leve durante a prova, assuma que todas as formas normais são com respeito ao ideal $I$. Assuma também que $f=f_{I}+N(f)$ e $f^{\prime}=f_{I}^{\prime}+N\left(f^{\prime}\right), \operatorname{com} f_{I}, f_{I}^{\prime}$ em $I$.

Primeiro, note que $N\left(f-f^{\prime}\right)=N(f)-N\left(f^{\prime}\right)$ pelo Corolário 3.1.8. Então temos que 


$$
\begin{aligned}
f+I=f^{\prime}+I & \Leftrightarrow \\
f-f^{\prime} \text { pertence a } I & \Leftrightarrow \\
N\left(f-f^{\prime}\right)=0 & \Leftrightarrow \\
N(f)-N\left(f^{\prime}\right)=0 & \Leftrightarrow \\
N(f)=N\left(f^{\prime}\right) &
\end{aligned}
$$

o que encerra a demonstração.

Corolário 3.1.10 Podemos definir uma estrutura multiplicativa em $N$ da seguinte forma: dados $f, f^{\prime}$ em $N$, definimos $f \cdot N f^{\prime}=N\left(f \cdot R f^{\prime}\right)$. Então, $N$, munido dessa estrutura multiplicativa é uma álgebra (possivelmente nula).

Ademais, $N$ juntamente com essa multiplicação é isomorfo a $R / I$ como álgebra.

Demonstração. Primeiro vejamos que $N$ é uma álgebra. Mas, por definição, $N$ é um espaço vetorial sobre $K$. Com isso, resta-nos verificar a associatividade da multiplicação, as leis distributivas envolvendo a multiplicação definida no enunciado do corolário e a existência de unidade para essa multiplicação.

As provas da associatividade e das leis distributivas decorrem do Corolário 3.1 .8 e são deixadas a cargo do leitor. A forma normal da unidade de $R$ é a unidade da multiplicação definida em $N$ (a verificação desse fato é simples).

Para verificar o isomorfismo, defina

$$
\begin{aligned}
\varphi: & R / I \longrightarrow N \\
& f+I \mapsto N(f)
\end{aligned}
$$

Primeiro note que, pelo Corolário 3.1.9, a função $\varphi$ definida acima é independente de representante. Para verificar que ela é um isomorfismo, precisamos verificar que é um homomorfismo de anéis, que ela é $K$-linear e que é bijetora. As duas primeiras verificações são conseqüências imediatas do Corolário 3.1.8, da definição de multiplicação em $N$ e do fato de $N(1)$ ser a unidade de $N$. Portanto nos voltamos para mostrar que $\varphi$ é bijetora.

Note que $\varphi$ é injetora pelo Corolário 3.1.9. A verificação de que $\varphi$ é sobrejetora é imediata: se $g$ é um elemento de $N$, então $g+I$ pertence a $\varphi^{-1}(g)$.

O próximo conceito será bastante útil para as demonstrações de muitos resultados importantes do restante da teoria.

Definição 3.1.5 (Elemento reduzido) Seja $R$ uma álgebra com uma base multiplicativa $\mathcal{B}, \leq$ uma ordem admissivel em $\mathcal{B}$ e $A$ um subconjunto de $R$. Um elemento $s \in A$ é reduzido com respeito a $A$ se

1. O coeficiente líder de sé igual a 1 . 
2. $\operatorname{supp}(s) \backslash\{\widehat{s}\}$ é um subconjunto de Norm(A).

Seguem alguns resultados interessantes sobre elementos reduzidos e que nos serão bastante úteis.

Lema 3.1.11 Seja $R$ uma álgebra com uma base multiplicativa $\mathcal{B}, \leq$ uma ordem admissivel em $\mathcal{B}$ e I um ideal de $R$. Sejam s; $s^{\prime}$ elementos reduzidos com respeito a um ideal $I$ de $R$. Então $\widehat{s}=\widehat{s^{\prime}}$ se e somente se $s=s^{\prime}$

Demonstração. É claro que $s=s^{\prime}$ implica $\widehat{s}=\widehat{s}^{\prime}$. Portanto, nos resta mostrar a outra implicação.

Assuma que $\widehat{s}=\widehat{s^{\prime}}$. Então o elemento $s-s^{\prime}$ pertence a $I$ (por esse ser um ideal). Contudo, como $\widehat{s}=\widehat{s^{\prime}}, \operatorname{cl}(s)=\operatorname{cl}\left(s^{\prime}\right)=1$ e $s$ e $s^{\prime}$ são reduzidos, teremos

$$
s-s^{\prime}=\widehat{s}+\sum_{\substack{\lambda_{i} \in K, b_{i} \in \operatorname{Norm}(\mathrm{I})}} \lambda_{i} b_{i}-\left(\widehat{s}+\sum_{\substack{\lambda_{j} \in K, b_{j} \in \operatorname{Norm}(\mathrm{I})}} \lambda_{j} b_{j}\right)
$$

Suponha então que $s-s^{\prime}$ é não nulo. Então, $s-s^{\prime}$ possui um termo líder. Logo, teremos, pela expressão acima, que $\widehat{s-s^{\prime}} \in(\operatorname{supp}(s) \backslash\{\widehat{s}\}) \cup\left(\operatorname{supp}\left(s^{\prime}\right) \backslash\left\{\widehat{s^{\prime}}\right\}\right) \subseteq \operatorname{Norm}(\mathrm{I})$ e o termo líder de $s-s^{\prime}$ pertence ao conjunto dos termos normais com respeito a $I$, uma contradição. Logo, $s-s^{\prime}=0$, donde $s=s^{\prime}$, como queríamos.

Lema 3.1.12 Seja $R$ uma álgebra com uma base multiplicativa $\mathcal{B}, \leq$ uma ordem admissivel em $\mathcal{B}$ e $I$ um ideal de $R$. Se $f$ é um elemento qualquer de $I$, então existe um elemento reduzido $s$ de $I$ tal que $\widehat{f}=\widehat{s}$.

Demonstração. A prova segue por contradição. Se o resultado é falso, então existe um conjunto $C$ de elementos de $I$ para os quais não existe um elemento reduzido de mesmo termo líder. Podemos escolher um elemento $f$ de $C$ que possua termo líder mínimo dentre todos os elementos de $C$ (afinal, os termos líderes estão bem ordenados pela ordem admissível fixada).

Se $f$ é reduzido, temos uma contradição e nada mais resta a demonstrar; então assuma que $f$ não é reduzido. Considere o conjunto dos elementos de $\mathcal{B}$ pertencentes ao suporte de $f$ e que são termos líderes de outros elementos de $I$, diferentes de $f$. Mais precisamente, podemos definir o conjunto:

$$
X_{f}:=(\operatorname{supp}(f) \cap \widehat{I}) \backslash\{\widehat{f}\}
$$

Se $X_{f}$ é vazio, basta dividirmos $f$ por $c l(f)$ e teremos um elemento reduzido, o que leva a unia contradição com as hipóteses iniciais e encerra a demonstração do lema. Suponha então que $X_{f}$ não é vazio.

Mas então, como $X_{f}$ é subconjunto do suporte de $f$, ele é finito. Ademais, todo elemento de $X_{f}$ é menor que $\widehat{f}$. Tome então $X_{f}=\left\{b_{1}, b_{2}, \ldots, b_{k}\right\}$. Pela hipótese de minimalidade, existem elementos reduzidos de $I, s_{1}, s_{2}, \ldots, s_{k}$, tais que $\widehat{s_{i}}=b_{i}$, para $1 \leq i \leq k$. Assim se tomarmos o seguinte elemento (cada $\lambda_{i}, 1 \leq i \leq k$ é o coeficiente de $b_{i}$ em $f$ ): 


$$
f^{\prime}=f-\lambda_{1} s_{1}-\lambda_{2} s_{2} \cdots-\lambda_{k} s_{k}
$$

teremos que $X_{f^{\prime}}:=\left(\operatorname{supp}\left(f^{\prime}\right) \cap \widehat{I}\right) \backslash\left\{\widehat{f}^{\prime}\right\}$ é vazio (note que cada subtração elimina um elemento de $\widehat{I}$ do suporte de $f$ e não pode introduzir novos elementos de $\widehat{I}$ ao suporte de $f^{\prime}$, por se tratar de um elemento reduzido). Com isso, $\frac{1}{c l(f)} f^{\prime}$ é um elemento reduzido com termo líder igual a $\widehat{f}$, o que encerra a demonstração.

Para maior praticidade, resumimos os dois resultados anteriores no seguinte corolário.

Corolário 3.1.13 Seja $R$ uma álgebra com uma base multiplicativa $\mathcal{B}$ sobre a qual está fixada uma ordem admissivel e seja $I$ um ideal de $R$. Então, para cada elemento $f$ de $I$, existe um único elemento reduzido $s$ tal que $\widehat{s}=\widehat{f}$.

Equivalentemente, para todo $m$ em $\widehat{I}$, existe um único elemento reduzido $s$ tal que $\widehat{s}=m$.

Encerramos esta seção com um lema que descreve a forma de elementos reduzidos.

Lema 3.1.14 Seja $R$ uma álgebra com uma base multiplicativa $\mathcal{B}$ sobre a qual está fixada uma ordem admissivel, $I$ um ideal de $R$ e $s$ um elemento reduzido com respeito a $I$. Então $s$ é da forma $s=\widehat{s}-n$, onde $n$ é a forma normal de $\widehat{s}$.

Demonstração. Se $s$ é um elemento reduzido de $I$, ele é, por definição, da forma $s=\widehat{s}+m$, com $m$ em Norm(I). Mas então, $\widehat{s}=s-m$ e a forma normal de $\widehat{s}$ (denotada no enunciado do lema por $n$ ) é $-m$ pelo Teorema 3.1.6.

\subsection{Bases de Gröbner}

Continuamos com a notação da seção anterior: seja $R$ uma álgebra com base multiplicativa $\mathcal{B}$ sobre a qual está fixada uma ordem admissível $\leq$. Apresentamos então a definição formal de Base de Gröbner de um ideal.

Definição 3.2.1 (Base de Gröbner) Seja $R$ uma álgebra com base multiplicativa $\mathcal{B}$ sobre a qual está fixada uma ordem admissivel $\leq$ e seja $I$ um ideal de $R$. Então uma Base de Gröbner para $I$ (com respeito a $\mathcal{B}$ e a $\leq$ ) é um subconjunto $G$ de $I$ tal que

$$
\langle\widehat{G}\rangle=\langle\widehat{I}\rangle
$$

Essa definição é equivalente a exigir que, para todo $f$ não nulo em $I$, exista $g$ em $G$ tal que $\widehat{g}$ divide $\widehat{f}$.

A partir de sua definição, podemos ver que a existência de Bases de Gröbner não é um problema. uma vez que todo ideal é uma Base de Gröbner para si próprio.

Uma observação importante feita para Bases de Gröbner comutativas e que deve ser lembrada também para o caso não comutativo é que uma Base de Gröbner para um ideal $I$ não é uma base de $I$ como espaço vetorial. Na realidade, em geral, podemos encontrar Bases de Gröbner para $I$ 
que não são sequer conjuntos geradores para $I$ como espaço vetorial. ${ }^{4}$ Por outro lado, toda Base de Gröbner para $I$ gera $I$ como ideal, como nos mostra o seguinte resultado.

Proposição 3.2.1 Seja $R$ uma álgebra com base multiplicativa $\mathcal{B}$ sobre a qual está fixada uma ordem admissivel $\leq$, seja I um ideal de $R$ e $G$ uma Base de Gröbner para $I$. Então G gera I como ideal.

Demonstração. A prova segue por contradição. Suponha que o resultado seja falso e tome um contra exemplo $f$ de termo líder mínimo. Mas então, pela definição de Base de Gröbner, existe $g$ em $G$ tal que $\widehat{f}=b \widehat{g} b^{\prime}$ para alguns $b, b^{\prime}$ em $\mathcal{B}$. Logo, o elemento

$$
f^{\prime}:=f-\frac{c l(f)}{c l(g)} b g b^{\prime}
$$

está em $I$ e temos que $\widehat{f}^{\prime}$ é menor que $\widehat{f}$ ou $f^{\prime}=0$. Mas se $f^{\prime}$ é nulo, $f$ é múltiplo de $g$ e não há mais o que mostrar. Por outro lado, se $f^{\prime} \neq 0$, esse está no ideal gerado por $G$ pela minimalidade de $\widehat{f}$. Mas então,

$$
f=f^{\prime}+\frac{c l(f)}{c l(g)} b g b^{\prime} \in I
$$

e chegamos a uma contradição, com a hipótese de que $f$ não pertence a $\langle G\rangle$.

Em vista desse resultado, freqüentemente nos referiremos simplesmente a uma Base de Gröbner $G$, o que quer dizer que $G$ é uma Base de Gröbner para o ideal gerado por $G$.

A partir da definição de Base de Gröbner, podemos ver que um ideal pode possuir muitas Bases de Gröbner e, muitas vezes, estas podem ser maiores que o desejável (até mesmo infinitas). O leitor deve recordar de nossa discussão sobre Teoria de Bases de Gröbner comutativas que, apesar disso, no caso comutativo temos sempre a garantia de que todo ideal admite (para cada ordem admissível dada) ao menos uma Base de Gröbner finita. Tal garantia é dada pela demonstração apresentada na Seção 2.4 para o Teorema da Base de Hilbert (Teorema 2.4.2) e encontra suas raízes no Lema de Dickson (Teorema 2.4.1).

Infelizmente, ao generalizarmos a teoria para o caso não comutativo, essa garantia é perdida. De fato, ideais (monomiais ou não) podem não ser finitamente gerados no caso não comutativo, o que elimina qualquer esperança de encontrar Bases de Gröbner finitas para eles.

Ademais, podemos encontrar ideais finitamente gerados para os quais não existe nenhuma Base de Gröbner finita, independente da ordem admissível fixada em $\mathcal{B}$. Ainda, existem ideais finitamente gerados que não admitem nenhuma Base de Gröbner finita para uma escolha de ordem admissível em $\mathcal{B}$, mas possuem Base de Gröbner finita para outra escolha de ordem. Exemplos para esses importantes fatos são fornecidos ao final da seção, nos Exemplos 3.2.2 e 3.2.3.

Então buscamos alguns casos particulares de Bases de Gröbner mais interessantes. O seguinte resultado é consequiência imediata da Definição 3.2.1 e nos permite eliminar elementos de uma Base de Gröbner.

\footnotetext{
${ }^{4} \mathrm{Em}$ vista dessa observação, encontra-se em alguns trabalhos o termo "conjunto gerador de Gröbner", em vez de "Base de Gröbner" [FFG93]. Contudo, optamos pela nomenclatura dada aqui (mesmo correndo o risco de causar alguma confusāo) por ela ser muito bem estabelecida na área.
} 
Afirmação 3.2.2 Seja $G$ uma Base de Gröbner para um ideal I de $R$. Se g é um elemento de $G$ tal que $\langle\widehat{G \backslash\{g}\}\rangle=\langle\widehat{G}\rangle$ (ou, equivalentemente, se existe $g^{\prime} \neq \mathrm{g}$ em $G$ tal que $\widehat{g^{\prime}}$ divide $\widehat{g}$ ), então $G \backslash\{g\}$ é uma Base de Gröbner para $I$.

No que segue, faremos uma breve digressão a fim de utilizar a idéia da afirmação acima para encontrar Bases de Gröbner com um número menor de elementos e, em certo sentido, mais simples. Para tanto, é preciso estudar uma forma de encontrar uma Base de Gröbner $G$ tal que $\widehat{G}$ seja "o menor possível".

Note que $\langle\widehat{G}\rangle$ é, por definição, gerado por elementos de $\mathcal{B}$, o que nos leva a estudar as propriedades de ideais dessa forma. Faremos então uso do conceito e de alguns resultados interessantes envolvendo ideais monomiais.

Definição 3.2.2 (Ideal Monomial) Seja $R$ uma álgebra com base multiplicativa $\mathcal{B}$. Um ideal I de $R$ é dito monomial se ele for gerado por elementos de $\mathcal{B}$ (como ideal).

No caso em que I é um ideal monomial, diremos que um conjunto gerador composto apenas por elementos de $\mathcal{B}$ é um conjunto gerador monomial.

Ainda, dado um ideal monomial I e um conjunto gerador monomial A para I, dizemos que $A$ é um conjunto gerador monomial minimal para I se nenhum subconjunto próprio de A gera $I$.

Na Proposição 3.2.9, mostraremos que conjuntos geradores monomiais minimais existem e são únicos. Uma vez que tivermos esse resultado demonstrado, poderemos definir Bases de Gröbner bastante especiais para o ideal $I$.

Os dois lemas seguintes refletem propriedades bem conhecidas dos ideais monomiais e serão utilizados livremente no texto.

Lema 3.2.3 Seja $A \subseteq \mathcal{B}$ e tome $m$ um monômio em $\langle A\rangle$. Então, existe $m^{\prime}$ em $A$ tal que $m^{\prime}$ divide $m$.

Lema 3.2.4 Seja I um ideal monomial e $f$ em I tal que $f=\sum_{i=1}^{k} \alpha_{i} b_{i}$, com $\alpha_{i} \in K$ e $b_{i} \in \mathcal{B}$, para $1 \leq i \leq k$. Então cada $b_{i}$ pertence a $I$, para $1 \leq i \leq k$.

O lema a seguir é de verificação simples, mas será usado em muitas ocasiões.

Lema 3.2.5 Se $R$ é uma álgebra com uma base multiplicativa $\mathcal{B}$ munida de uma ordem admissivel e I é um ideal de $R$, então, para todo monômio $m$ no ideal gerado pelos termos líderes de $I,\langle\widehat{I}\rangle$, existe um elemento $f$ de $I$ tal que $\widehat{f}=m$.

Demonstração. Tome um monômio qualquer $m$ em $\langle\widehat{I}\rangle$. Como $m$ é um monômio e é um elemento do ideal gerado por $\widehat{I}$, segue que existe um elemento $m_{i} \in \widehat{I}$ tal que $m_{i}$ divide $m$, ou seja $m=b m_{i} b^{\prime}$, para alguns $b, b^{\prime} \in \mathcal{B}$. Mas então, como $m_{i} \in \widehat{I}$, segue que existe $f \in I$ tal que $\widehat{f}=m_{i}$. Assim, se definimos $f^{\prime}:=b f b^{\prime}$, teremos que $\widehat{f}^{\prime}=m$, uma vez que $b \widehat{f} b^{\prime}=b m_{i} b^{\prime} \neq 0$. É claro que $f^{\prime}$ pertence a $I$, o que encerra a demonstração.

O seguinte resultado é conseqüència imediata dos lemas acima.

Corolário 3.2.6 Se I é um ideal monomial, então $I=\langle\widehat{I}\rangle$. 
O seguinte lema é de cunho mais técnico e será utilizado diretamente na demonstração da Proposição 3.2.9.

Lema 3.2.7 Seja $R$ uma álgebra com base multiplicativa $\mathcal{B}$ munida de uma ordem admissível $\leq$. Seja $A$ um subconjunto não vazio de $\mathcal{B}$. Então existe pelo menos um elemento $m$ de $A$ com a seguinte propriedade:

Se existe $m^{\prime}$ em A tal que $m^{\prime}$ divide $m$, então $m^{\prime}=m$.

Demonstração. Uma vez que $\mathcal{B}$ é um conjunto bem ordenado pela ordem admissível fixada, $A$ possui um menor elemento $m$. Portanto, $m \leq b$, para todo $b$ em $A$. Mostraremos que $m$ é um elemento com a propriedade destacada no enunciado do lema.

Tome agora $m^{\prime}$ em $A$ tal que $m^{\prime}$ divide $m$. Pela definição de ordem admissível, $m^{\prime} \leq m$ e concluímos que $m^{\prime}=m$.

Lema 3.2.8 Seja B um conjunto não vazio de monômios. Então B é um conjunto gerador monomial minimal para $\langle B\rangle$ se e somente se não existem elementos distintos $b$ e $b^{\prime}$ em $B$ de forma que $b$ divide $b^{\prime}$.

Demonstração. Tome $B \subseteq \mathcal{B}$ de forma que $B$ é um conjunto gerador monomial minimal para $\langle B\rangle$. Neste caso, se existem $b$ e $b^{\prime}$ em $B$ como no enunciado do lema, $B \backslash\left\{b^{\prime}\right\}$ é um subconjunto próprio de $B$ e gera $\langle B\rangle$, nos levando a uma contradição com a hipótese de que $B$ é um conjunto gerador monomial minimal.

Para a implicação reversa, assuma que $B$ é um conjunto não vazio de monômios e que não existem $b$ e $b^{\prime}$ distintos em $B$ tais que $b$ divide $b^{\prime}$. Claro que $B$ é um conjunto gerador monomial para $\langle B\rangle$. Resta mostrar que nenhum subconjunto próprio de $B$ gera $\langle B\rangle$.

Mas, se $b$ pertence a $B, b$ não é divisível por nenhum elemento de $B \backslash\{b\}$, donde segue que $b$ não pertence a $\langle B \backslash\{b\}\rangle$. Portanto, $B$ é um conjunto gerador monomial minimal para $\langle B\rangle$.

Proposição 3.2.9 Seja $R$ uma álgebra com base multiplicativa $\mathcal{B}$ munida de uma ordem admissível. Então, se I é um ideal monomial de $R, I$ possui um único conjunto gerador monomial minimal.

Demonstração. Temos duas coisas a demonstrar: primeiro mostraremos que $I$ de fato possui um conjunto gerador monomial minimal e, em seguida, provaremos que ele é único.

A fim de mostrar a existència de um conjunto gerador monomial minimal para $I$, considere $A$ como sendo o conjunto de todos os monòmios de $I$. Seja então

$$
M:=\left\{m \text { em } A \mid \text { se } m^{\prime} \in A \text { divide } m \text {, então } m^{\prime}=m\right\}
$$

Note que, como $I$ é um ideal monomial, $A$ é não vazio. Ademais, $A \subseteq \mathcal{B}$ por definição. Logo, segue do Lema 3.2.7 que $M$ é não vazio.

Afirmamos que $M$ é um conjunto gerador monomial minimal para $I$. O conjunto $M$ é composto apenas por monòmios e está contido em $I$ por definição. Ainda, segue da 
definição de $M$ que não existem elementos distintos $b$ e $b^{\prime}$ em $M$ tais que $b$ divide $b^{\prime}$. Logo, pelo Lema 3.2.8, $M$ é um conjunto gerador monomial minimal para $\langle M\rangle$.

Resta-nos então mostrar que $\langle M\rangle=I$. Naturalmente $\langle M\rangle \subseteq I$; para ver a inclusão reversa, basta tomar $I_{M}$ um conjunto gerador monomial de $I$ (ele existe pelo fato de $I$ ser monomial) e mostrar que $I_{M} \subseteq\langle M\rangle$. Consideramos então $i$ um elemento qualquer de $I_{M}$ e definimos

$$
D_{i}:=\{m \in A \mid m \text { divide } i \text { e } m \neq i\}
$$

Uma propriedade interessante do conjunto $D_{i}$ é que, para todo $a \in D_{i}$, todos os elementos de $A$ que dividem $a$ também pertencem a $D_{i}$ (pela transitividade da divisão).

Por um lado, se $D_{i}$ é vazio, $i \in M$ por definição. Por outro lado, se $D_{i}$ é não vazio, pelo Lema 3.2.7, existe um elemento $m_{i} \in D_{i}$ que não possui divisores diferentes de si próprio dentre os elementos de $D_{i}$. Isso, em vista do comentário acima, implica que $m_{i}$ não possui divisores diferentes de si próprio dentre os elementos de $A$, donde $m_{i} \in M$ pela definição do conjunto $M$. Logo, $i \in\langle M\rangle$ e concluímos que $\langle M\rangle=\left\langle I_{M}\right\rangle=I$, como queríamos.

Para ver que $I$ possui um único conjunto monomial minimal, tome $M$ e $M^{\prime}$ dois conjuntos geradores monomiais minimais distintos para $I$. Mostraremos que isso nos leva a uma contradição.

Podemos supor, sem perda de generalidade, que existe $m$ em $M$, tal que $m$ não pertence a $M^{\prime}$. Mas como $m$ é um monômio de $I$ e $M^{\prime}$ é um conjunto gerador para $I$, segue que existe um elemento $m^{\prime}$ em $M^{\prime}$ que divide $m$. Analogamente, existe $m^{\prime \prime}$ em $M$ de forma que $m^{\prime \prime}$ divide $m^{\prime}$. Mas então, ou $m^{\prime \prime} \neq m$ e temos uma contradição com a hipótese de $M$ ser um conjunto gerador monomial minimal para $I$ (pelo Lema 3.2.8) ou $m^{\prime \prime}=m=m^{\prime}$, contradizendo a hipótese de que $m$ não pertence a $M^{\prime}$.

O seguinte corolário segue imediatamente da Proposição 3.2.9 e do Lema 3.2.8.

Corolário 3.2.10 Seja B um conjunto não vazio de monômios. Então $B$ é o conjunto gerador monomial minimal de $\langle B\rangle$ se e somente se não existem $b$ e $b^{\prime}$ distintos em $B$ de forma que $b$ divide $b^{\prime}$.

Ajustando o coeficiente líder de cada elemento de uma Base de Gröbner e utilizando o fato de que todo ideal monomial possui um único conjunto gerador monomial minimal, chegamos ao conceito de Base de Gröbner minimal.

Definição 3.2.3 (Base de Gröbner Minimal) Uma Base de Gröbner minimal para um ideal $I$ de $R$ é uma Base de Gröbner $G$ para I satisfazendo:

1. Para todo g em $G$, o coeficiente líder de g é 1.

2. O conjunto $\widehat{G}$ é o conjunto gerador monomial minimal de $\langle\widehat{G}\rangle$. 
Mostraremos adiante que todo ideal possui pelo menos uma Base de Gröbner minimal. Infelizmente, um ideal pode ter muitas Bases de Gröbner minimais, e elas podem ser maiores que o desejável, como nos mostra o seguinte exemplo.

Exemplo 3.2.1 Considere o alfabeto $X=\{a, b\}$, a álgebra livre sobre $X, K\langle X\rangle$, e tome o conjunto de todas as palavras sobre $X$ como uma base multiplicativa de $K\langle X\rangle$.

Definimos então uma ordem admissível $\leq \mathrm{em} X^{*}$. Primeiro, dada uma letra $v \in X \mathrm{e}$ uma palavra $p \in X^{*}$, Lembramos que $\|p\|_{v}$ é o grau de $v$ em $p$ (ou seja, o número de ocorrências da letra $v$ na palavra $p$ ). Assim, dados dois elementos $p, p^{\prime}$ de $X^{*}$, diremos que $p<p^{\prime}$ se

$$
\begin{aligned}
& \|p\|_{a}<\left\|p^{\prime}\right\|_{a} \text { ou } \\
& \left.\|p\|_{a}=\left\|p^{\prime}\right\|_{a} \text { e } p \text { é menor que } p^{\prime} \text { de acordo com a ordem deglex (com } a>b\right)
\end{aligned}
$$

Afirmamos que essa ordem é admissível e fornecemos uma demonstração para esse fato na Afirmação 3.2.11 a seguir.

Consideramos então o ideal $I$ de $K\langle X\rangle$ gerado por $B:=\left\{a^{3}, b^{2}\right\}$. Logo, como $I$ é monomial, segue do Corolário 3.2.6 que $I=\langle\widehat{I}\rangle$ e $B$ é um conjunto gerador de $\langle\widehat{I}\rangle$.

Assim, pelo Corolário 3.2.10, $B$ é o conjunto gerador monomial minimal para $\langle\widehat{I}\rangle$. Com isso, cada elemento da família

$$
\left(G_{i}:=\left\{a^{3}-b^{2 i}, b^{2}\right\}\right)_{i \in \mathbb{N}^{*}}
$$

constitui uma Base de Gröbner minimal (finita) para $I$.

Por outro lado, note que a união de todas essas Bases de Gröbner:

$$
G:=\bigcup_{i \in \mathbb{N}^{*}} G_{i}
$$

constitui uma Base de Gröbner minimal infinita para $I$.

Afirmação 3.2.11 A ordem $\leq$ sobre $X^{*}=\{a, b\}^{*}$ definida no Exemplo 3.2.1 é admissivel.

Demonstração. Precisamos mostrar que $\leq$ é uma boa ordem e que a condição 2' da Afirmação 3.1.1 é satisfeita.

Vejamos primeiro que $\leq$ é uma boa ordem. Tome $A$ um subconjunto qualquer de $X^{*}$. Mostraremos que $A$ possui um menor elemento. Com efeito, seja

$$
\text { ९: } \begin{aligned}
A \longrightarrow \mathbb{N} \\
p \longmapsto\|p\|_{a}
\end{aligned}
$$

Naturalmente, o conjunto $\operatorname{Im}(\varphi)$ é bem ordenado, de forma que podemos considerar seu menor elemento $m$. Pela definição de $\leq$ vemos que $p<p^{\prime}$, para todo elemento $p \mathrm{~cm}$ $p^{-1}(m)$ e todo $p^{\prime} \in A \backslash \varphi^{-1}(m)$. Dessa forma, se encontrarmos o menor elemento do conjunto $\varphi^{-1}(m)$, ele será o menor elemento de $A$. 
Mas, como os elementos de $\varphi^{-1}(m)$ possuem mesmo grau (com relação a $a$ ), temos que eles são ordenados pela ordem deglex, que é uma boa ordem. Com isso $\varphi^{-1}(m)$ possui um menor elemento e $\leq$ é uma boa ordem.

A verificação da propriedade 2' é imediata e deixada a cargo do leitor.

Contudo, dado um ideal $I$, podemos destacar uma Base de Gröbner minimal especial que é, em certo sentido, melhor que as demais. Sua definição é a seguinte.

Definição 3.2.4 (Base de Gröbner Reduzida) Uma Base de Gröbner reduzida para um ideal I de $R$ é uma Base de Gröbner $G$ para I satisfazendo:

1. G é uma Base de Gröbner minimal.

2. Todo elemento de $G$ é reduzido.

Em seguida, mostramos que todo ideal possui uma única Base de Gröbner reduzida.

Proposição 3.2.12 Seja $R$ uma álgebra com base multiplicativa $\mathcal{B}$ sobre a qual está fixada uma ordem admissivel $\leq$. Seja I um ideal de $R$. Então I possui uma única Base de Gröbner reduzida (com respeito $a \leq$ ).

Demonstração. Considere o ideal gerado pelos termos líderes de $I,\langle\widehat{I}\rangle$. Naturalmente esse ideal é monomial e, portanto, possui um único conjunto gerador monomial minimal (que denotaremos por $M$ ). Tome então $m$ um monômio qualquer em $M$. Pelo Lema $3.2 .5, m$ pertence a $\widehat{I}$; logo, pelo Corolário 3.1 .13 , existe um único elemento reduzido $s$ de $I$ tal que $\widehat{s}=m$. Definimos então o seguinte conjunto:

$$
G:=\{s \mid s \text { é reduzido com respeito a } I \text { e } \widehat{s} \in M\}
$$

Então, p'elos comentários acima, existe um elemento em $G$ para cada $m$ em $M$, donde $\widehat{G}=M$ e $G$ é uma Base de Gröbner minimal (lembramos que o coeficiente líder de todo elemento reduzido é 1 por definição). Além disso, como todo elemento de $G$ é reduzido, ela é reduzida.

A unicidade de $G$ segue diretamente do Corolário 3.1 .13 e da unicidade de $M$.

Corolário 3.2.13 Seja $R$ uma álgebra com base multiplicativa $\mathcal{B}$ sobre a qual está fixada uma ordem admissivel $\leq$. Seja I um ideal de R. Então I possui ao menos uma Base de Gröbner minimal.

O seguinte resultado é consequiência imediata da Proposição 3.2.12 e do Lema 3.1.14.

Corolário 3.2.14 Seja $R$ uma álgebra com base multiplicativa $\mathcal{B}$ sobre a qual está fixada uma ordem admissivel $\leq$. Seja $I$ um ideal de $R$ e seja $M$ o conjunto gerador monomial minimal de $\langle\widehat{I}\rangle$. Então a Base de Gröbner reduzida para I (com respeito $a \leq$ ) é:

$$
G=\{m-N(m) \mid m \in M\}
$$

Em particular, $\operatorname{card}(G)=\operatorname{card}(M)$. 
É interessante notar que, em certo sentido, se $I$ é um ideal, a Base de Gröbner reduzida para $I$, $G$, é a Base de Gröbner "mais enxuta" possível. A partir do corolário acima, não é difícil ver que o número de elementos de qualquer Base de Gröbner para $I$ terá pelo menos card $(G)$ elementos. Além disso, se $G^{\prime}$ é uma Base de Gröbner para $I$, da definição de conjunto gerador monomial minimal e de sua unicidade, segue que $\widehat{G} \subseteq \widehat{G^{\prime}}$.

Esses comentários nos levam ao seguinte corolário.

Corolário 3.2.15 Seja $R$ uma álgebra com base multiplicativa $\mathcal{B}$ sobre a qual está fixada uma ordem admissivel $\leq$. Seja I um ideal de $R$ e seja $G$ a Base de Gröbner reduzida para $I$. Então, se $G^{\prime} e ́$ uma Base de Gröbner para $I, \widehat{G} \subseteq \widehat{G^{\prime}}$.

Em particular, I possui uma Base de Gröbner finita se e somente se a Base de Gröbner reduzida para I é finita.

Antes de encerrar a seção, retomamos a discussão sobre existência de Bases de Gröbner finitas para um ideal. Apresentamos em seguida o exemplo prometido que exibe um ideal finitamente gerado que não admite nenhuma Base de Gröbner finita (com respeito a uma ordem admissível dada). O exemplo ilustra também o importante fato de que essa característica do ideal depende da ordem escolhida. Com efeito, o ideal apresentado abaixo não admite nenhuma Base de Gröbner finita para uma dada ordem, mas admite uma Base de Gröbner com apenas um elemento para outra escolha de ordem admissível.

Exemplo 3.2.2 Seja $X=\{a, b\}$ e considere a álgebra livre sobre $X, K\langle X\rangle$, com base multiplicativa $X^{*}$. Fixe ainda a ordem de grau e lexicografia em $X^{*} \operatorname{com} a>b$. Tome então $f=a b a-b^{2} a$ e considere o ideal $I=\langle f\rangle$. Então a Base de Gröbner reduzida para $I$ é: ${ }^{5}$

$$
G:=\left\{a b^{2 i-1} a-b^{2 i} a \mid i \geq 1\right\}
$$

Por outro lado, se considerarmos a ordem de grau e lexicografia em $X^{*} \operatorname{com} b>a$, teremos que o conjunto $\{f\}$ é uma Base de Gröbner para $I$. $\Delta$

O seguinte exemplo foi daclo por Mansson e Nordbeck (Exemplo 2 de [MN02]) e apresenta um ideal finitamente gerado que não admite Base de Gröbner finita para nenhuma escolha de ordem admissível.

Exemplo 3.2.3 (Mansson e Nordbeck, 2002) Seja $I$ o ideal de $K\langle a, x, y\rangle$ gerado por $\{a x y a-x y a, x y-y x\}$. Então o conjunto

$$
G:=\left\{a y^{i} x^{i} a-y^{i} x^{i} a, x y-y x \mid i \geq 1\right\}
$$

é a Base de Gröbner reduzida para $I$ para qualquer ordem admissível satisfazendo $x y>$ $y x .{ }^{6}$ Um argumento análogo é válido no caso em que $x y<y x$, levando à construção de uma Base de Gröbner reduzida infinita para $I$.

\footnotetext{
${ }^{5}$ Detalhes desta construção podem ser vistos no Exemplo 4.2.6.

${ }^{6}$ As ferramentas que permite verificar esse fato serão apresentadas na Seção 4.2.
} 
Resumimos essas importantes observações a seguir para futura referência.

Observação 3.2.16 É possivel encontrar uma álgebra $R$ com uma base multiplicativa $\mathcal{B}$ e um ideal finitamente gerado I de $R$, tais que:

- Existe uma ordem admissível em $\mathcal{B}, \leq$, de forma que I não possui nenhuma Base de Gröbner finita com respeito $a \leq$.

- Existe uma ordem admissivel $\leq 1$ (diferente de $\leq$ ) em $\mathcal{B}$, tal que I admite uma Base de Gröbner finita com respeito $a \leq$.

É possivel encontrar uma álgebra $R$ com uma base multiplicativa $\mathcal{B}$ e um ideal finitamente gerado $I$ de $R$, de forma que $I$ não admite nenhuma Base de Gröbner finita, independente da ordem admissivel fixada em $\mathcal{B}$.

\section{3 Álgebras de Dimensão Finita}

Nesta seção apresentaremos três resultados envolvendo álgebras de dimensão finita. Primeiro, mostraremos que se um ideal $I$ de uma álgebra (finitamente gerada) $R$ é tal que $R / I$ é de dimensão finita, então $I$ possui uma Base de Gröbner finita (Proposição 3.3.2). Não exploraremos maiores conseqüências ou aplicações desse fato, mas referimos o leitor interessado para [Gre00].

Em seguida, introduziremos a noção de Base de Gröbner universal e mostraremos que todo ideal nas condições acima admite uma Base de Gröbner universal finita.

Finalmente, mostraremos que, uma vez fixado um corpo, existem apenas finitas álgebras (não isomorfas) de uma dada dimensão que admitem teoria de Bases de Gröbner (Proposição 3.3.8).

Inicialmente, precisamos mostrar que se $R$ é finitamente gerada como álgebra, então a base $\mathcal{B} \cup\{0\}$ é finitamente gerada como semigrupo.

Lema 3.3.1 Seja $R$ uma álgebra e $\mathcal{B}$ uma base multiplicativa de $R$. Se a álgebra $R$ é finitamente gerada (como álgebra), então $\mathcal{B} \cup\{0\}$ é finitamente gerado como semigrupo.

Demonstração. Como $R$ é finitamente gerada, podemos tomar um conjunto finito $S=$ $\left\{f_{1}, f_{2}, \ldots, f_{n}\right\}$ que gera $R$ como álgebra. Considere então o conjunto $B=\cup_{i=1}^{n} \operatorname{supp}\left(f_{i}\right)$. Como $S$ é um conjunto finito, teremos que o conjunto $B$ também é finito. Afirmamos que $B$ gera $\mathcal{B} \cup\{0\}$ como semigrupo. Para verificar esse fato, precisamos mostrar que um elemento $b$ qualquer de $\mathcal{B}$ se escreve como produto de elementos de $B$. Mas, como $b$ é um elemento da álgebra $R$, ele se escreve como

$$
b=\sum_{i=1}^{k} \lambda_{i} f_{i_{1}} f_{i_{2}} \cdots f_{i_{n(2)}}
$$

Claro que cada $f_{i_{j}}$ se escreve como combinação linear de elementos de $\mathcal{B}$ e podemos expandir cada $f_{i_{j}}$ na soma acima. Mas então, os únicos elementos de $\mathcal{B}$ que surgirão desta expansão serão produtos de elementos de $B$. Como se trata de uma base, temos que $b$ deve ser igual a somente um dos monômios que aparece nessa expansão. 
Ainda, note que se $\mathcal{B} \cdot \mathcal{B} \nsubseteq \mathcal{B}$, o zero é gerado por $B$, dado que existem dois monômios em $\mathcal{B}$ (gerados por $B$ ) cujo produto é zero. Por outro lado, se $\mathcal{B} \cdot \mathcal{B} \subseteq \mathcal{B}$, podemos incluir o zero no conjunto $B$.

Com isso, podemos apresentar o principal resultado desta seção.

Proposição 3.3.2 Seja $R$ uma álgebra finitamente gerada com base multiplicativa $\mathcal{B}$ sobre a qual está fixada uma ordem admissivel. Se I é um ideal de $R$ tal que $R / I$ é de dimensão finita, então $I$ possui uma Base de Gröbner finita.

Demonstração. Note primeiramente que, pelo Corolário 3.1.10, span(Norm(I)) é isomorfo a $R / I$, e concluímos que Norm(I) é uma base de $R / I$ (como espaço vetorial) e é, portanto, um conjunto finito.

Em seguida, podemos utilizar a Proposição 3.2 .9 para ver que $\langle\widehat{I}\rangle$ possui um único conjunto gerador monomial minimal. Denotemos tal conjunto por $M$ e vejamos que $M$ é um conjunto finito.

Mas, pelo Lema 3.3.1, segue que $\mathcal{B} \cup\{0\}$ possui um conjunto gerador finito (como semigrupo); denotemo-lo por $B$. Considere agora

$$
\mathcal{T}:=\{b c \mid b \in B \text { e } c \in \operatorname{Norm}(\mathrm{I})\} \cup B
$$

Pelas observações acima, $\mathcal{T}$ é um subconjunto finito de $\mathcal{B}$. Afirmamos que $M$ está contido em $\mathcal{T}$. Para ver esse fato, tome $m$ em $M$. Como $M$ é um subconjunto de $\mathcal{B}$, segue que $m$ se escreve como um produto de elementos não nulos de $B: m=b_{1} b_{2} \cdots b_{k}$.

Se $k=1$, segue que $m$ está em $B \subseteq \mathcal{T}$. Por outro lado, se $k>1$, segue que $m=b_{1} c$, com $b_{1}$ em $B$ e $c$ em $\mathcal{B}$. Porém, como $c$ divide $m$ e $M$ é o conjunto gerador monomial minimal de $\langle M\rangle=\langle\widehat{I}\rangle$, segue do Corolário 3.2 .10 que $c$ não pertence a $\widehat{I}$. Logo, acabamos de mostrar que $M$ está contido em $\mathcal{T}$, donde $M$ é um conjunto finito.

Finalmente, se utilizarmos o Corolário 3.1.13, podemos encontrar um elemento reduzido para cada elemento de $M$; tais elementos constituem uma Base de Gröbner reduzida finita para $I$.

Bases de Gröbner Universais Vejamos em seguida que um ideal $I$ nas condições acima admite mais que uma Base de Gröbner finita: ele admite uma Base de Gröbner universal finita.

Definição 3.3.1 (Base de Gröbner Universal) Seja $R$ uma álgebra que admite teoria de Bases de Gröbner. Fixamos uma base multiplicativa $\mathcal{B}$ de $R$ e tomamos $I$ um ideal de $R$. Uma Base de Gröbner universal para $I$ (com respeito à base $\mathcal{B}$ ) é um subconjunto $G$ de $I$ com a propriedade de que $G$ é uma Base de Gröbner para I, para qualquer ordem admissivel fixada em $\mathcal{B}$.

Denotamos por $d=\operatorname{dim}_{K}(R / I)$ e seja $B=\left\{b_{1}, b_{2}, \ldots, b_{x}\right\}$ um conjunto gerador para $\mathcal{B} \cup\{0\}$ (como semigrupo). Claro que sua finitude é garantida pelo Lema 3.3.1.

Definimos então a extensão de um monomio qualquer $m$ (com respeito a $B$ ) como sendo o menor inteiro $i$ tal que $m$ pode ser escrito como produto de $i$ elementos de $B$ e o denotamos por 
$\mathrm{e}(m)$. Com isso, a extensão de um elemento está bem definida e não é difícil de ver que dois monômios de extensões diferentes são distintos.

Chamamos a atenção do leitor para o fato de que a noção de extensão não concorda com as noções de grau de palavras ou de caminhos na álgebra livre ou na álgebra de caminhos, respectivamente. Com efeito, a palavra vazia pertence a qualquer conjunto gerador de $X^{*}$ como semigrupo, donde e $(1)=1 \mathrm{com}$ respeito a qualquer conjunto gerador de $X^{*}$.

Lema 3.3.3 Para toda ordem admissivel fixada em $\mathcal{B}$, para todo monômio $m$ de extensão maior que $d+1$, existe um elemento $f$ de $I$, tal que:

1. O termo líder de $f$ divide $m$.

2. Cada elemento b do suporte de $f$ é tal que $\mathrm{e}(b) \leq d+1$.

Demonstração. Tome um monômio $m \operatorname{com~} \mathrm{e}(m)>d+1$. Então $m$ se escreve como $m=b_{i_{1}} b_{i_{2}} \cdots b_{i_{\mathrm{e}(m)}}$. Considere o conjunto

$$
C:=\left\{m_{k}:=b_{i_{1}} b_{i_{2}} \cdots b_{i_{k}} \mid 0<k \leq d+1\right\}
$$

Afirmamos que $\mathrm{e}\left(m_{k}\right)=k$, para todo $0<k \leq d+1$. De fato, pela definição de extensão, $\mathrm{e}\left(m_{k}\right) \leq k$. Por outro lado, se $\mathrm{e}\left(m_{k}\right)<k$, podemos reescrever $m_{k}$ como produto de $h$ geradores de $B$, com $h<\mathrm{e}\left(m_{k}\right)$, o que nos permite reescrever $m$ como produto de $j$ geradores, com $j<\mathrm{e}(m)$, nos levando a uma contradição.

Portanto, $m_{i} \neq m_{j}$, se $i \neq j$ e o conjunto $C$ contém $d+1$ elementos.

Seja agora $\pi: R \longrightarrow R / I$ a projeção canônica e considere

$$
L=\left(\pi\left(m_{1}\right), \pi\left(m_{2}\right), \ldots, \pi\left(m_{k}\right)\right)
$$

Como temos $d+1>\operatorname{dim}_{K}(R / I)$ elementos em $L$, existem coeficientes $\alpha_{1}, \alpha_{2}, \ldots, \alpha_{k}$ não todos nulos, tais que $\sum_{i=1}^{k} \alpha_{i} \pi\left(m_{i}\right)=0$ (note que não estamos assumindo que $\pi\left(m_{i}\right) \neq \pi\left(m_{j}\right)$, se $\left.i \neq j\right)$.

Com isso, $f:=\sum_{i=1}^{k} \alpha_{i} m_{i}$ pertence a $I$ e é não nulo (pelo fato de os monômios que aparecem nessa soma serem distintos). Por outro lado, é claro que cada $m_{i}$ divide $m$ e $\mathrm{e}\left(m_{l}\right) \leq d+1$.

Assim, se tomarmos uma ordem admissível qualquer em $\mathcal{B}$, o termo líder de $f$ será igual a $m_{i}$, para algum $i$ e $\widehat{f}$ divide $m$.

Lema 3.3.4 Para toda ordem admissivel $\leq$ fixada em $\mathcal{B}$, se $m$ é um monômio de extensão maior que $d+1$, entäo existe um elemento $f$ de $I$ tal que $\widehat{f}=m$ e, para todo b em $\operatorname{supp}(f) \backslash\{\widehat{f}\}$, $\mathrm{e}(b) \leq d+1$.

Demonstração. Denotemos por $M$ o subconjunto de $\mathcal{B}$ formado pelos elementos de extensão menor ou igual a $d+1$ e suponha que o lema seja falso. Então existem uma ordem admissível $\leq$ em $\mathcal{B}$ e um monômio $m$, de modo que $m$ não satisfaz o lema com a ordem $\leq$ fixada em $\mathcal{B}$. 
Fixamos então a ordem $\leq$ em $\mathcal{B}$ e tomamos um monômio $m$ que não satisfaz o lema e que seja mínimo (segundo $\leq$ ) no conjunto de todos os monômios que não satisfazem o lema.

Mas então, como e $(m)>d+1$, segue do Lema 3.3.3 que existe $h$ em $I$ de forma que $\widehat{h}$ divide $m$ e $\operatorname{supp}(h) \subseteq M$. Portanto, existem monômios $a$ e $b$, tais que $m=a \widehat{h} b$. Consideramos:

$$
x:=m-\frac{1}{c l(h)} a h b
$$

Note que $\operatorname{supp}(x)=\operatorname{supp}(a h b) \backslash\{m\}$. Ademais, é claro que, para todo $z \operatorname{em~} \operatorname{supp}(x)$, $z<m$.

Por um lado, $\operatorname{se} \operatorname{supp}(x)$ estiver contido em $M$, tomamos $f=x$ e temos uma contradição com a suposição de $m$ não satisfazer o lema.

Assuma, portanto, que $\operatorname{supp}(x) \nsubseteq M$. Logo, existem monômios $a_{1}, a_{2}, \ldots, a_{l}$ de extensão maior que $d+1$ em $\operatorname{supp}(x)$.

Mas então, podemos utilizar a hipótese de minimalidade sobre $m$ para concluir que, para cada $a_{i}(1 \leq i \leq l)$, existe um elemento $h_{i}$ em $I$ tal que $\widehat{h_{i}}=a_{i}$ e $\operatorname{supp}\left(h_{i}\right) \backslash\left\{\widehat{h_{i}}\right\} \subseteq M$. Claro que todo elemento em $\bigcup_{i=1}^{l} \operatorname{supp}\left(h_{i}\right)$ é menor que $m$. Tomamos então:

$$
f:=a h b-\sum_{i=1}^{l} \frac{1}{c l\left(h_{i}\right)} h_{i}
$$

Pelo argumento acima, $\widehat{f}=a \widehat{h} b=m$. Ainda, cada $a_{i}$ é cancelado na soma acima, fazendo com que $\operatorname{supp}(f) \backslash\{\widehat{f}\}$ esteja contido em $M$. Finalmente, como $h_{i}$ pertence a $I$, segue que $f$ pertence a $I$ e chegamos a uma contradição com a hipótese do lema ser falso.

Com isso, podemos apresentar a seguinte proposição.

Proposição 3.3.5 Seja $R$ uma álgebra que admite teoria de Bases de Gröbner e tomemos $\mathcal{B}$ uma base multiplicativa de $R$. Se I é um ideal de $R$ tal que $R / I$ é de dimensão finita, então I admite uma Base de Gröbner universal finita (com respeito à base $\mathcal{B}$ ).

Demonstração. Denotamos por $M$ o subconjunto de $\mathcal{B}$ formado somente por monômios de extensão menor ou igual a $d+1$. Não é difícil ver que $M$ é um conjunto finito. Com isso, definimos:

$$
X=\{f \in I \mid \operatorname{supp}(f) \subseteq M\}
$$

e

$$
Y=\bigcup_{f \in X} \operatorname{supp}(f)
$$


Pelo Lema 3.3.3, $X$ e $Y$ são conjuntos não vazios. Note ainda que $Y \subseteq M$, e concluímos que $Y$ é um conjunto finito. Portanto, as partes de $Y$ constituem um conjunto finito. Seja agora

$$
C:=\{c \in \mathcal{P}(Y) \mid \text { existe } f \in I \operatorname{com} \operatorname{supp}(f)=c\}
$$

Tome, para cada $c \in C$, um elemento $g_{c}$ de $I$ tal que $\operatorname{supp}\left(g_{c}\right)=c$ e defina $G$ como sendo o conjunto formado por todos os elementos $g_{c}$ assim escolhidos. Claro que $G$ é um subconjunto finito de $I$. Mostraremos em seguida que ele é uma Base de Gröbner universal para $I$.

Para tanto, tome uma ordem admissível qualquer em $\mathcal{B}$ e um elemento arbitrário $f$ de $I$. Mostraremos que $\widehat{f}$ é divisível pelo termo líder de algum elemento de $G$. Mas então temos três casos:

1. O suporte de $f$ está contido em $Y^{7}$

Em outras palavras, todo elemento de $\operatorname{supp}(f)$ tem extensão menor ou igual a $d+1$. Neste caso, existe algum $g_{c}$ em $G$ tal que $\operatorname{supp}\left(g_{c}\right)=\operatorname{supp}(f)$, donde $\widehat{g_{c}}=\widehat{f}$ na ordem admissível qualquer fixada.

2. A extensão de $\widehat{f}$ é maior que $d+1$.

Neste caso, pelo Lema 3.3.3 existe um elemento $g$ de $G$, de forma $\widehat{g}$ divide $\widehat{f}$ na ordem admissível fixada.

3. A extensão de $\widehat{f}$ é menor ou igual a $d+1$ e $f$ não está contido em $Y$.

Neste caso, existem elementos $a_{1}, a_{2}, \ldots, a_{k} \operatorname{em} \operatorname{supp}(f)$ com extensão maior que $d+1$. Mas, pelo Lema 3.3.4, para cada monômio $a_{i}$, existe um elemento $h_{i}$ em $I$, satisfazendo o seguinte:

- $\widehat{h_{i}}=a_{i}$.

- $\operatorname{supp}\left(a_{i}-\frac{1}{c l\left(h_{i}\right)} h_{i}\right) \subseteq M$.

- Para cada $b$ em $\operatorname{supp}\left(a_{i}-\frac{1}{c l\left(h_{i}\right)} h_{i}\right), b<a_{i}<\widehat{f}$.

Assim, tomamos:

$$
f^{\prime}:=f-\sum_{i=1}^{k} \frac{1}{\operatorname{cl}\left(h_{i}\right)} h_{i}
$$

Note que cada $a_{i}$ é cancelado na soma acima, de modo que $\operatorname{supp}\left(f^{\prime}\right)$ está contido em $M$. Ainda, como cada $h_{i}$ pertence a $I$, temos que $f^{\prime}$ pertence a $I$, donde $\operatorname{supp}\left(f^{\prime}\right) \in C$. Finalmente, note que $\hat{f}=\widehat{f}^{\prime}$, uma vez que todos os monomios que aparecem na soma $\sum_{i=1}^{k} \frac{1}{c l\left(h_{i}\right)} h_{i}$ são estritamente menores que $\widehat{f}$ (pelo fato de cada $a_{i}$ ser estritamente menor que $\widehat{f}$ ).

Portanto, existe $g$ em $G$, de modo que $\operatorname{supp}(g)=\operatorname{supp}\left(f^{\prime}\right)$, encerrando a demonstração.

\footnotetext{
${ }^{7} \mathrm{Em}$ vista da definição de $Y$ e do fato de $f$ ser um elemento de $I$, essa condição é equivalente a exigir que o suporte de $f$ esteja contido em $M$.
} 
Finitude de $A B G_{K}(n)$ Voltamo-nos então para mostrar que, fixados um corpo $K$ e uma dimensão $n$ existe apenas um número finito de $K$-álgebras não isomorfas com essa dimensão que admitem teoria de Bases de Gröbner.

Assim, fixe um corpo $K$ e uma dimensão $n$. Seja $\operatorname{Alg}_{K}(n)$ a classe de todas as álgebras de dimensão $n$ sobre $K$ que admitem bases multiplicativas. Então, temos o seguinte resultado (sua demonstração é bem natural, e é conseqüência imediata do fato de $\mathcal{B}_{1}$ e $\mathcal{B}_{2}$ serem conjuntos geradores de $A_{1}$ e $A_{2}$, respectivamente, de forma que a omitiremos aqui).

Lema 3.3.6 Sejam $A_{1}$ e $A_{2}$ em $\operatorname{Alg}_{K}(n)$, com bases multiplicativas $\mathcal{B}_{1}$ e $\mathcal{B}_{2}$. Se existe um isomorfismo de semigrupos

$$
\phi: \mathcal{B}_{1} \cup\{0\} \longrightarrow \mathcal{B}_{2} \cup\{0\}
$$

então as álgebras $A_{1}$ e $A_{2}$ são isomorfas.

Seja agora $X$ um conjunto finito com $n$ elementos, isto é, $X:=\left\{e_{1}, e_{2}, \cdots, e_{n}\right\}$ e denote por $T:=X \times X \times X$.

Dada uma base multiplicativa $\mathcal{B}$ e uma bijeção $\pi_{\mathcal{B}}: \mathcal{B} \longrightarrow X$, podemos definir uma aplicação $\tau_{\pi_{\mathcal{B}}}: \mathcal{B} \times \mathcal{B} \longrightarrow T$, da seguinte forma:

$$
\tau_{\pi_{\mathcal{B}}}\left(b_{1}, b_{2}\right):=\left(\pi_{\mathcal{B}}\left(b_{1}\right), \pi_{\mathcal{B}}\left(b_{2}\right), \pi_{\mathcal{B}}\left(b_{1} b_{2}\right)\right)
$$

Com isso, podemos demonstrar os seguinte resultado.

Lema 3.3.7 Sejam $\mathcal{B}_{1}$ e $\mathcal{B}_{2}$ duas bases multiplicativas de álgebras $A_{1}$ e $A_{2}$, respectivamente. Então, se existem bijeções $\pi_{\mathcal{B}_{1}}: \mathcal{B}_{1} \longrightarrow X \quad$ e $\pi_{\mathcal{B}_{2}}: \mathcal{B}_{2} \longrightarrow X$ tais que

$$
\tau_{\pi_{\mathcal{B}_{1}}}\left(\mathcal{B}_{1} \times \mathcal{B}_{1}\right)=\tau_{\pi_{\mathcal{B}_{2}}}\left(\mathcal{B}_{2} \times \mathcal{B}_{2}\right)
$$

então $A_{1}$ é uma álgebra isomorfa a $A_{2}$.

Demonstração. Pelo Lema 3.3.6, é suficiente exibir um isomorfismo de semigrupos entre $\mathcal{B}_{1} \cup\{0\}$ e $\mathcal{B}_{2} \cup\{0\}$. Defina então

$$
\begin{aligned}
\phi: & \mathcal{B}_{1} \cup\{0\} \longrightarrow \mathcal{B}_{2} \cup\{0\} \\
& b \mapsto \pi_{\mathcal{B}_{2}}^{-1}\left(\pi_{\mathcal{B}_{1}}(b)\right)
\end{aligned}
$$

Então teremos que

$$
\phi\left(b_{1}\right) \cdot \phi\left(b_{2}\right)=\pi_{\mathcal{B}_{2}}^{-1}\left(\pi_{\mathcal{B}_{1}}\left(b_{1}\right)\right) \cdot \pi_{\mathcal{B}_{2}}^{-1}\left(\pi_{\mathcal{B}_{1}}\left(b_{2}\right)\right)=b_{1}^{\prime} \cdot b_{2}^{\prime}
$$

para alguns $b_{1}^{\prime}$ e $b_{2}^{\prime} \mathrm{em} \mathcal{B}_{2}$. Ademais, valem as seguintes propriedades:

$$
\begin{aligned}
& \pi_{\mathcal{B}_{2}}\left(b_{1}^{\prime}\right)=\pi_{\mathcal{B}_{1}}\left(b_{1}\right) \mathrm{e} \\
& \pi_{\mathcal{B}_{2}}\left(b_{2}^{\prime}\right)=\pi_{\mathcal{B}_{1}}\left(b_{2}\right)
\end{aligned}
$$


Ma então, pela hipótese de que $\tau_{\pi_{\mathcal{B}_{1}}}\left(\mathcal{B}_{1} \times \mathcal{B}_{1}\right)=\tau_{\pi_{\mathcal{B}_{2}}}\left(\mathcal{B}_{2} \times \mathcal{B}_{2}\right)$ e pela construção das funções $\tau_{\pi_{\mathcal{B}}}$, não é difícil ver que $\pi_{\mathcal{B}_{2}}\left(b_{1}^{\prime} b_{2}^{\prime}\right)=\pi_{\mathcal{B}_{1}}\left(b_{1} b_{2}\right)$. Mas então, teremos

$$
\phi\left(b_{1} b_{2}\right)=\pi_{\mathcal{B}_{2}}^{-1}\left(\pi_{\mathcal{B}_{1}}\left(b_{1} b_{2}\right)\right)=b_{1}^{\prime} b_{2}^{\prime}
$$

e segue que $\phi$ é homomorfismo de semigrupos, como queríamos.

Suponha, portanto, que temos uma seqüencia infinita de álgebras de $A \lg _{K}(n)$, duas a duas não isomorfas, e que admitem bases multiplicativas

$$
S=\left(A_{i}\right)_{A_{i} \in \operatorname{Alg}_{K}(n)}
$$

Escolha uma base multiplicativa $\mathcal{B}_{i}$ para cada uma das álgebras $A_{i}$ e uma bijeção $\pi_{\mathcal{B}_{i}}$ dessa base $\operatorname{com} X$.

Como essas álgebras são duas a duas não isomorfas, segue do Lema 3.3 .7 que $\operatorname{Im}\left(\tau_{\pi_{\mathcal{B}_{i}}}\right) \neq$ $\operatorname{Im}\left(\tau_{\pi_{\mathcal{B}_{j}}}\right)$, para todos $A_{i}$ e $A_{j}$ na seqüência $S$. Por outro lado, $\operatorname{Im}\left(\tau_{\pi_{\mathcal{B}_{i}}}\right)$ é um subconjunto de $T$, para todo $A_{i}$ em $S$. Claro que $T$ é um conjunto finito e chegamos a uma contradição com a hipótese de que não existem $A_{i}$ e $A_{j}$ não isomorfos na seqüência infinita $S$.

Portanto, podemos enunciar a seguinte proposição.

Proposição 3.3.8 Dado um corpo $K$ e uma dimensão $n$, existe um número finito de álgebras sobre $K$ e de dimensão $n$ que admitem teoria de Bases de Gröbner, a menos de isomorfismo.

Não é difícil ver que se $A_{1}$ e $A_{2}$ são duas álgebras isomorfas, então $A_{1}$ admite teoria de Bases de Gröbner se e somente se $A_{2}$ também admite teoria de Bases de Gröbner. Com isso, podemos definir $A B G_{K}(n)$ como sendo o conjunto das classes de isomorfismo (de álgebra) de $A l g_{K}(n)$, consistindo de álgebras que admitem teoria de Bases de Gröbner.

Com essa terminologia, a Proposição 3.3.8 nos mostra que $A B G_{K}(n)$ é um conjunto finito, para todo $K$ e todo $n$.

Comentamos ainda que é sabido que $\operatorname{Alg}_{K}(n)$ é uma variedade algébrica afim de $K^{3 n}$, que $G L(n)$ age em $\operatorname{Alg}_{K}(n)$ e que cada órbita corresponde a uma classe de isomorfismo de $\operatorname{Alg}_{K}(n)$ [LRS95]. Assim, $A B G_{K}(n)$ pode ser visto como um subconjunto finito de $\operatorname{Alg}_{K}(n) / G L(n)$.

\subsection{Propriedades Básicas e Álgebras de Caminhos}

Assumiremos as mesmas hipóteses que temos tomado até o presente momento, ou seja, $R$ é uma álgebra finitamente gerada sobre um corpo $K$, que possui uma base multiplicativa $\mathcal{B}$ sobre a qual está fixada uma ordem admissível $\leq$.

O propósito desta seção é apresentar uma série de propriedades comuns a toda álgebra nas hipóteses acima. Essa caracterização nos levará à interessante conclusão de que, na realidade, toda álgebra nessas condições pode ser vista como um quociente de uma álgebra de caminhos. As principais idéias contidas aqui foram originalmente exploradas por Farkas, Feustel e Green [FFG93] e foram refinadas posteriormente. A abordagem dada aqui é muito semelhante à apresentada por Green [Gre00].

Em particular, é interessante mencionar que é possivel encontrar em qualquer álgebra $R$ que admita uma teoria de Bases de Gröbner elementos com propriedades muito parecidas com aquelas 
dos vértices e arcos em álgebras de caminhos. Com efeito, a busca por tais elementos consiste na essência do que é feito nesta seção e eles são a chave para o estabelecimento de um isomorfismo entre $R$ e um quociente de uma álgebra de caminhos.

\subsubsection{Resultados Iniciais}

Recordamos que um elemento qualquer $f$ de $R$ é idempotente se $f^{2}=f$. Além disso, um subconjunto $A$ de $R$ é dito ortogonal se $f \cdot f^{\prime}=0$, para todos os elementos $f$ e $f^{\prime}$ distintos de $A$.

Iniciamos então nossa discussão com a observação trivial de que a unidade de $R$ se escreve como combinação linear de elementos da base $\mathcal{B}$ (não estamos assumindo que 1 é um elemento de $\mathcal{B}$ ). Com isso, podemos escrever

$$
1=\sum_{k=1}^{n} \alpha_{k} v_{k}
$$

onde cada $\alpha_{k}$ é um escalar não nulo e $v_{k}$ (para $1 \leq k \leq n$ ) são elementos distintos de $\mathcal{B}$.

$O$ primeiro resultado que apresentamos traz algumas propriedades interessantes desses elementos $v_{k}$. Posteriormente ficará claro que podemos estabelecer uma correspondência desses elementos com vértices de uma álgebra de caminhos.

Lema 3.4.1 O conjunto $\left\{v_{1}, v_{2}, \ldots, v_{n}\right\}$ é um conjunto ortogonal de idempotentes e cada $\alpha_{k}=1$

Demonstração. Precisamos demonstrar três coisas:

1. O produto $v_{i} \cdot v_{j}=0$, se $i \neq j$.

2. Para todo $1 \leq i \leq n, v_{i}=v_{i}^{2}$.

3. Cada $\alpha_{i}=1$.

Para ver a primeira parte, tomemos dois índices quaisquer $i, j$ (entre 1 e $n$ ) distintos. Assumimos então que $v_{i} v_{j} \neq 0$ e mostraremos que isso nos leva a uma contradição.

Como a base $\mathcal{B}$ é multiplicativa e os elementos $v_{i}$ e $v_{j}$ pertencem a ela, temos que o produto $v_{i} v_{j}=b$ pertence a $\mathcal{B}$ (por estarmos assumindo que o produto $v_{i} v_{j}$ é não nulo). Naturalmente $b \neq v_{i}$ ou $b \neq v_{j}$, uma vez que assumimos que os elementos $v_{i}$ e $v_{j}$ são distintos. Podemos assumir por hora que $b \neq v_{i}$. Então, utilizando a Equação 3.1, teremos:

$$
v_{i}=v_{i} \cdot 1=v_{i}\left(\sum_{k=1}^{n} \alpha_{k} v_{k}\right)=\sum_{k=1}^{n} \alpha_{k} v_{i} v_{k}
$$

Em particular, $v_{i} v_{j}=b$ aparece na soma acima. Por outro lado, essa soma é uma combinação linear de elementos da base $\mathcal{B}$. Mas $v_{i}$ só pode ser escrito de uma única maneira como combinação linear de elementos de $\mathcal{B}: 1 \cdot v_{i}$ (dado que $v_{i} \in \mathcal{B}$ ).

Assim, como assumimos que $b \neq v_{i}$, temos que o coeficiente de $b$ na soma acima deve ser zero. Mas como nenhum $\alpha_{i}$ é zero por hipótese (fizemos essa suposição ao apresentar a Equação 3.1), temos que existe algum $l \neq j$ de forma que $v_{i} v_{l}=b=v_{i} v_{j}$. 
Mas, ou $v_{j}<v_{l}$ ou $v_{l}<v_{j}$. Se assumimos que $v_{j}<v_{l}$, utilizamos o fato de a ordem ser admissível para concluir que $b=v_{i} v_{j}<v_{i} v_{l}=b$, um absurdo. Analogamente, se $v_{l}<v_{j}$, teremos que $v_{i} v_{l}<v_{i} v_{j}$, nos levando a uma contradição com a suposição de que $v_{i} v_{j} \neq 0$. Podemos utilizar argumentos análogos para obter contradições semelhantes às acima para o caso em que $b \neq v_{j}$. Assim, concluímos que $v_{i} v_{j}=0$.

Podemos utilizar o fato que acabamos de demonstrar na equação acima para concluir que $v_{i}=\alpha_{i} v_{i} v_{i}$, donde segue que $v_{i}=v_{i}^{2}$ e $\alpha_{i}=1$, para cada $1 \leq i \leq n$.

A seguinte proposição nos mostra uma propriedade muito interessante das bases multiplicativas munidas de ordens admissíveis. Em particular, ela justifica a hipótese feita na Seção 3.1 de que a multiplicação em $\mathcal{B}$ é reflexiva.

Proposição 3.4.2 Seja $R$ uma álgebra com base multiplicativa $\mathcal{B}$ sobre a qual está fixada uma ordem admissivel $\leq$. Então, se $b$ é um elemento de $\mathcal{B}$, existem únicos $1 \leq i, j \leq n$ tais que $v_{i} b=b$ $e b v_{j}=b$.

Ademais, dado um índice $1 \leq l \leq n$, teremos:

- Se l é diferente de $i$, então $v_{l} b=0$.

- Se l é diferente de $j$, então $b v_{l}=0$.

Demonstração. Mostraremos o argumento para $j$; o outro caso é completamente análogo. Utilizando a Equação 3.1 e o Lema 3.4.1, temos:

$$
b=b \cdot 1=b\left(\sum_{k=1}^{n} v_{k}\right)
$$

Porém, como $b$ é um elemento da base $\mathcal{B}$, existe uma única forma de escrevê-lo como combinação linear de elementos de $\mathcal{B}: b=1 \cdot b$. Com isso, $b$ deve aparecer em pelo menos um dos termos na soma acima.

De fato, se isso não ocorre, conseguimos escrever $b$ como combinação linear de elementos de $\mathcal{B}$ distintos de $b$, donde $\mathcal{B}$ é linearmente dependente. Portanto, existe $j$ tal que $b v_{j}=b$.

Vejamos agora que $b v_{l}=0$ se $l \neq i$. Mas então, basta utilizar a primeira parte:

$$
b v_{l}=\left(b v_{j}\right) v_{l} \stackrel{\text { Lema }}{=} 3.4 .10
$$

Temos então a segunda parte do lema demonstrada. Resta-nos apresentar o argumento para o fato de $j$ ser único.

Mas, se isso não for verdade, teremos $l \neq j$ tal que $b v_{l}=b$, o que é impossível pela segunda parte do lema (naturalmente $b \neq 0$ por ser um elemento de uma base).

Com esse resultado em mãos, podemos definir os conceitos de origem e término de um elemento da base $\mathcal{B}$. 
Definição 3.4.1 (Origem e Término) Seja $R$ uma álgebra com base multiplicativa $\mathcal{B}$ sobre a qual está fixada uma ordem admissível $\leq$. Se $b$ é um elemento de $\mathcal{B}$, definimos a origem de $b$ como sendo o único elemento idempotente $\mathrm{o}(b)$ de $\mathcal{B}$ tal que $\mathrm{o}(b) \cdot b=b$. Analogamente, definimos como término de $b$ o único elemento idempotente de $\mathcal{B} \mathrm{t}(b)$ tal que $b \cdot \mathrm{t}(b)=b$.

Deve estar claro para o leitor que no caso em que $R$ é uma álgebra de caminhos (com base $\mathcal{P}$ fixada), as noções de origem e término vistas na Definição 3.4 .1 coincidem com as noções de origem e término de caminhos em um grafo. Dessa forma, não faremos distinção entre elas.

Ao longo de todo trabalho, temos feito largo uso da observação envolvendo divisões entre elementos de $\mathcal{B}$ feita imediatamente antes da Proposição 3.4.2. Destacamos esse comentário no seguinte corolário para maior conveniência do leitor.

Corolário 3.4.3 Seja $R$ uma álgebra com base multiplicativa $\mathcal{B}$ sobre a qual está fixada uma ordem admissivel $\leq$. Então a divisão é reflexiva em $\mathcal{B}$, no seguinte sentido: para todo a em $\mathcal{B}$, existem monômios $a_{1}$ e $a_{2}$ tais que $a=a_{1} a a_{2}$. Conseqüentemente, se $b$ e $b^{\prime}$ são elementos de $\mathcal{B}$, tais que $b$ divide $b^{\prime}$, então existem elementos $b_{1}$ e $b_{2}$ de $\mathcal{B}$, tais que $b^{\prime}=b_{1} b b_{2}$.

Demonstração. O resultado segue imediatamente do fato de que $b=\mathrm{o}(b) b \mathrm{t}(b)$.

Lema 3.4.4 Seja $b \in \mathcal{B}$. Então $b \geq \mathrm{o}(b)$ e $b \geq \mathrm{t}(b)$.

Demonstração. Da definição de $\mathrm{o}(b)$, temos que $b=\mathrm{o}(b) b$, donde $\mathrm{o}(b)$ divide $b$. O resultado segue então do fato de a ordem ser admissível.

$\mathrm{O}$ argumento para $\mathrm{t}(b)$ é análogo.

Em seguida, mostraremos que os únicos idempotentes em $\mathcal{B}$ são os elementos $v_{1}, v_{2}, \ldots, v_{n}$.

Corolário 3.4.5 Se $b \in \mathcal{B} \backslash\left\{v_{1}, v_{2}, \ldots, v_{n}\right\}$, então $b^{2} \neq b$.

Demonstração. Uma vez que $\mathcal{B}$ é uma base de $R$ como espaço vetorial, segue que 0 não pertence a $\mathcal{B}$. Portanto se $b^{2}=0$, não há mais o que demonstrar, pois $b \neq 0$ por pertencer a $\mathcal{B}$.

Suponha então que $b^{2} \neq 0$. Mas pelo fato de $b$ não ser um elemento de $\left\{v_{1}, v_{2}, \ldots, v_{n}\right\}$ e pelo Lema $3.4 .4, b>\mathrm{o}(b)$. Com isso, utilizamos o fato de $b^{2}$ ser não nulo e a presença de uma ordem admissível para concluir que $b^{2}>\mathrm{o}(b) b=b$, o que demonstra o resultado.

No que segue, chamaremos de $V$ o subconjunto de $\mathcal{B}$ formado pelos idempotentes de $\mathcal{B}$, ou seja $V:=\left\{v_{1}, v_{2}, \ldots, v_{n}\right\}$. Note o leitor que dada uma base $\mathcal{B}$ de uma álgebra $R$, os elementos $v_{i}$ $(1 \leq i \leq n)$ da Equação 3.1 estão unicamente determinados (pelo fato de $\mathcal{B}$ ser uma base de $R$ ). Com isso, o conjunto $V$ está unicamente determinado (uma vez fixada a base $\mathcal{B}$ ).

Antes de apresentar o próximo resultado, definimos as noções de elementos decomponíveis e indecomponíveis por produto. L'm elemento $b$ de $\mathcal{B}$ é dito decomponível por produto (ou simplesmente decomponível) se existem elementos $b_{1}$ e $b_{2}$ em $\mathcal{B} \backslash V$, tais que $b=b_{1} b_{2}$. Como esperado, diremos que um elemento $b$ de $\mathcal{B}$ é indecomponível por produto (ou simplesmente indecomponível) se ele não for decomponível por produto.

Lema 3.4.6 Cada elemento $v$ de $V$ é indecomponivel por produto. 
Demonstração. A prova segue por contradição. Suponha que $v \in V$ é decomponível por produto. Logo existem $b_{1}$ e $b_{2}$ em $\mathcal{B} \backslash V$ tais que $v=b_{1} b_{2}$. Mas então

$$
v=b_{1} b_{2}=\mathrm{o}\left(b_{1}\right) b_{1} b_{2}=\mathrm{o}\left(b_{1}\right) v
$$

Podemos utilizar o Lema 3.4.1 para concluir que $v=0$ se $v \neq \mathrm{o}\left(b_{1}\right)$ (o que nos leva a uma contradição). Por outro lado, se tomamos $v=\mathrm{o}\left(b_{1}\right)$, temos que $v$ divide $b_{1}$. Porém, $b_{1}$ divide $v$ por hipótese, donde $v=b_{1}$, uma contradição, pois, neste caso, $b_{1} \in V$.

Denotaremos pelo restante desta seção por $E$ o conjunto composto pelos elementos de $\mathcal{B} \backslash V$ que são indecomponíveis por produto. Conforme veremos mais adiante, os elementos desse conjunto serão identificados com arcos em uma álgebra de caminhos.

Assim, como é de se esperar, mostraremos (na Proposição 3.4.8) que todo elemento não idempotente de $\mathcal{B}$ se escreve como produto de elementos de $E$. Vale comentar também que, dada uma base $\mathcal{B}$, como $V$ está unicamente determinado (pelo fato de $\mathcal{B}$ ser um base), o conjunto $E$ também está unicamente determinado.

O próximo fato é uma conseqüência imediata da presença de uma ordem admissível e nos será muito útil na demonstração da Proposição 3.4.8.

Lema 3.4.7 Se $b_{1}$ e $b_{2}$ são elementos distintos de $\mathcal{B}$, então, para todo $b$ em $\mathcal{B}$, valem as seguintes implicações:

1. Se $b_{1} b=b_{2} b$, então $b_{1} b=b_{2} b=0$.

2. $S e b b_{1}=b b_{2}$, então $b b_{1}=b b_{2}=0$.

Demonstração. Demonstraremos a primeira implicação; a demonstração da segunda é completamente análoga. Podemos assumir sem perda de generalidade que $b_{1}<b_{2}$. Portanto, como temos uma ordem admissível, se as multiplicações $b b_{1}$ e $b b_{2}$ são não nulas, segue que $b_{1} b<b_{2} b$, nos levando a uma contradição com as hipóteses.

Proposição 3.4.8 Seja $R$ uma álgebra com base multiplicativa $\mathcal{B}$ sobre a qual está fixada uma ordem admissivel $\leq$. Seja $V$ o conjunto formado pelos idempotentes de $\mathcal{B}$ e $E$ o conjunto formado pelos elementos indecomponiveis por produto de $\mathcal{B} \backslash V$. Então todo elemento de $\mathcal{B} \backslash V$ é um produto da forma $b_{1} b_{2} \cdots b_{m}$, com $b_{i} \in E$, para $1 \leq i \leq m$.

Demonstração. Suponha que o resultado seja falso. Então existe um subconjunto não vazio $C$ de $\mathcal{B} \backslash V$ composto pelos elementos que não são indecomponíveis por produto (e, portanto, não pertencem a $E$ ) e nem se escrevem como um produto de elementos de E.

Devido à presença de uma boa ordem, $C$ possui um elemento mínimo. Chamemos um tal elemento de $b$. Se $b$ não puder ser escrito como produto de elementos de $\mathcal{B} \backslash V, b$ pertence a $E$ por definição, o que nos leva a uma contradição com o fato de $C$ ser não vazio e não há mais o que demonstrar.

Portanto, podemos assumir que $b=b_{1} b_{2}$, com $b_{1}$ e $b_{2}$ em $\mathcal{B} \backslash V$. Ainda, pela definição de ordem admissível, $b \geq b_{1}$ e $b \geq b_{2}$ (dado que tanto $b_{1}$ quanto $b_{2}$ dividem $b$ ). Mostraremos que $b \neq b_{1}$ e $b \neq b_{2}$. 
De fato, suponha que $b=b_{1}$. Então $b=b \mathrm{t}(b)$ e $b=b_{1} b_{2}=b b_{2}$, donde $b=b \mathrm{t}(b)=b b_{2}$. Agora, pelo Lema 3.4.7, se $\mathrm{t}(b)$ e $b_{2}$ são distintos então o produto $b \mathrm{t}(b)=b b_{2}=b$ é zero. Como isso não pode acontecer, concluímos que $b_{2}=\mathrm{t}(b)$. Porém, $\mathrm{t}(b) \in V$ e $b_{2} \notin V$, por suposição. Isso nos leva a uma contradição e concluímos que $b \neq b_{1}$. De forma análoga, $b \neq b_{2}$, e temos que $b>b_{1}$ e $b>b_{2}$.

Mas então, pela minimalidade de $b$, segue que tanto $b_{1}$ quanto $b_{2}$ se escrevem como um produto de elementos de $E$, donde

$$
b=b_{1} b_{2}=a_{1} a_{2} \cdots a_{k} a_{1}^{\prime} a_{2}^{\prime} \cdots a_{j}^{\prime}
$$

com $a_{i}$ e $a_{h}^{\prime}$ em $E$, para todos $1 \leq i \leq k$ e $1 \leq h \leq j$.

Logo, $b$ se escreve como um produto de elementos de $E$, o que contradiz a hipótese do conjunto $C$ ser não vazio.

O lema a seguir nos traz uma observação de verificação simples, mas que será importante nos argumentos que seguem.

Lema 3.4.9 Se $R$ é finitamente gerada, então E é finito.

Demonstração. Pelo Lema 3.3.1, sabemos que se $R$ é finitamente gerada, então $\mathcal{B} \cup\{0\}$ é finitamente gerado como semigrupo. Por outro lado, a partir da definição de elemento indecomponível por produto e pelas propriedades dos elementos idempotentes de $\mathcal{B}$, é imediato ver que $E$ está contido em qualquer conjunto gerador de $\mathcal{B} \cup\{0\}$, donde segue que $E$ é finito.

Vale comentar que no caso de $R$ ser uma álgebra de caminhos $K \Gamma$ (com a base $\mathcal{P}$ fixada), $V$ é exatamente o conjunto $V(\Gamma)$ e $E$ o conjunto $A(\Gamma)$. Esses fatos são verificados facilmente pelas propriedades das álgebras de caminhos que exploramos na Seção 1.4. Ainda, caso $R$ seja uma álgebra livre $K\langle X\rangle$ (com a base $X^{*}$ fixada), $V=\{1\}$ e $E=X$.

Com o que temos até aqui, podemos construir um grafo orientado $\Gamma$ a partir da álgebra $R$, da seguinte maneira. Tome o conjunto de vértices de $\Gamma$ como sendo $V(\Gamma)=V$. Ainda, um arco $e_{v_{1} v_{2}}$ com ponta inicial $v_{1}$ e ponta final $v_{2}$ pertence ao conjunto de arcos de $\Gamma$ quando existe um elemento $b \in E$ tal que o $(b)=v_{1}$ e t $(b)=v_{2}$. Uma vez que os conjuntos $E$ e $V$ estão unicamente determinados para uma base $\mathcal{B}$ fixada de $R$, então o grafo $\Gamma$ também está unicamente determinado (a menos de isomorfismo). Chamaremos o grafo $\Gamma$ de grafo associado a $\mathcal{B}$.

Seguimos com a introdução de alguns conceitos que serão necessários para enunciar o principal resultado desta seção.

Definição 3.4.2 (Binômio Puro) Seja $R$ uma álgebra qualquer com base $\mathcal{B}$. Um binômio puro é um elemento da forma $b-b^{\prime}$, com $b$ e $b^{\prime}$ em $\mathcal{B}$.

Um ideal de $R$ é dito puramente binomial se possuir um conjunto gerador composto somente por binômios puros.

Um conjunto gerador puramente binomial de um ideal é um conjunto gerador composto somente por binômios puros.

A definição a seguir é uma generalização do conceito de ideal puramente binomial. 
Definição 3.4.3 (Ideal 2-nomial) Seja $R$ uma álgebra qualquer com base $\mathcal{B}$. Um ideal $I$ de $R$ é 2-nomial se possuir um conjunto gerador cujos elementos são todos binômios puros ou monômios.

Em outras palavras, I é gerado por elementos da forma $p$ ou $p-q$, com $p, q$ em $\mathcal{B}$.

Um conjunto gerador 2-nomial de um ideal é um conjunto gerador composto somente por binômios puros ou monômios.

Definição 3.4.4 (Elemento Uniforme) Seja $R$ uma álgebra que admite teoria de Bases de Gröbner, $f$ um elemento não nulo de $R$ e considere o suporte de $f$, dado por $\operatorname{supp}(f)=$ $\left\{b_{1}, b_{2}, \ldots, b_{k}\right\}$. Dizemos que $f$ é uniforme se todo elemento de $\operatorname{supp}(f)$ possui a mesma origem e o mesmo término. Mais precisamente, $f$ é uniforme quando existem idempotentes o e $t$ de $\mathcal{B}$, de forma que o $\left(b_{i}\right)=o$ e $\mathrm{t}\left(b_{i}\right)=t$, para todo $1 \leq i \leq k$.

Ainda, um subconjunto $A$ de $R$ é uniforme quando todos os seus elementos forem uniformes.

A Seção 4.2 .1 se encarregará de apresentar alguns resultados importantes acerca de elementos uniformes. Contudo, adiaremos a exposição desses resultados para essa seção, uma vez que eles não serão necessários neste momento.

Por hora, mencionamos somente que todo ideal de uma álgebra de caminhos pode ser gerado por elementos uniformes (uma demonstração para esse fato é fornecida na Proposição 4.2.5) e apresentaremos um resultado que mostra que todo ideal 2-nomial possui um conjunto gerador 2nomial composto somente por elementos uniformes.

Por outro lado, vale comentar que nem todo ideal puramente binomial possui um conjunto gerador puramente binomial e uniforme, conforme será visto no Exemplo 4.2.1.

Lema 3.4.10 Todo ideal 2-nomial I pode ser gerado por um conjunto 2-nomial uniforme. Ademais, todo ideal 2-nomial I finitamente gerado pode ser gerado por um conjunto 2-nomial uniforme e finito.

Demonstração. Antes de mais nada, afirmamos que, se $I$ é um ideal e $a-b$ é um binômio puro não uniforme em $I$, então $a$ e $b$ pertencem a $I$. De fato, suponhamos que $\mathrm{o}(a) \neq \mathrm{o}(b)$. Mas então $\mathrm{o}(a)(a-b)=a$ e pertence a $I$. Da mesma forma, $\mathrm{o}(b)(a-b)=b$ pertence a $I$. O caso em que $\mathrm{t}(a) \neq \mathrm{t}(b)$ é análogo.

Tomamos então um conjunto gerador para $I$ da forma

$$
\mathcal{G}=\left\{a_{1}-b_{1}, a_{2}-b_{2}, \ldots, c_{1}, c_{2}, \ldots\right\}
$$

com $a_{i}, b_{i}$ e $c_{i}$ caminhos em $K \Gamma$, para todo $i>1$ (ele existe pelo fato de $I$ ser 2-nomial). Por um lado, é claro que cada $c_{i}$ é uniforme, restando-nos apenas analisar os elementos da forma $a_{i}-b_{i}$. Suponha então que existem elementos dessa forma que não são uniformes. Então o conjunto

$$
H:=\left\{a_{i_{j}}-b_{i_{j}} \mid a_{i_{j}}-b_{i_{j}} \in \mathcal{G} \text { e não é uniforme }\right\}
$$

é não vazio. Logo, pelo argumento acima, $a_{i}$, e $b_{i}$, pertencem a $I$, para todos $a_{i_{j}}-b_{i_{j}}$ em H. Assim,

$$
\mathcal{G}^{\prime}:=(\mathcal{G} \backslash H) \cup\left\{a_{i_{j}}, b_{i_{j}} \mid a_{i_{j}}-b_{i_{j}} \in H\right\}
$$


é um conjunto gerador para $I$ 2-nomial e uniforme. Ademais, como $I$ é finitamente gerado, $\mathcal{G}$ é finito, pois todo elemento de um conjunto gerador finito para $I$ pode ser gerado por um número finito de elementos de $\mathcal{G}$. Finalmente, é claro que $\mathcal{G}^{\prime}$ será finito se $\mathcal{G}$ o for.

Apresentamos então o principal resultado desta seção.

Teorema 3.4.11 (Green, 2000) Seja $R$ uma álgebra com uma base multiplicativa $\mathcal{B}$ munida de uma ordem admissivel $\leq$. Tome $\Gamma$ o grafo associado a $\mathcal{B}$ e considere a álgebra de caminhos $K \Gamma$ com base $\mathcal{P}$. Então existe um epimorfismo de $K$-álgebras $\phi: K \Gamma \longrightarrow R$ satisfazendo:

1. Se $p$ é um caminho em $\mathcal{P}$, então $\phi(p) \in \mathcal{B} \cup\{0\}$.

2. Se $b \in \mathcal{B}$, então existe um caminho $p$ em $\mathcal{P}$ tal que $\phi(p)=b$.

3. O núcleo da aplicação $\phi$ (que denotamos por I) é um ideal 2-nomial.

4. $O$ ideal I está contido em $J^{2}$ (o ideal de $K \Gamma$ gerado pelos caminhos de comprimento 2). Em particular, a imagem de cada arco e de cada vértice de $\Gamma$ pela aplicação $\phi$ é não nula.

5. Existe um conjunto gerador 2-nomial e uniforme de I.

Um elemento importante para mostrar a afirmação envolvendo ideais 2-nomiais feita no teorema é compreender a relação desses com o fato de $\mathcal{B}$ e $\mathcal{P}$ serem bases multiplicativas. ${ }^{8}$

De fato, podemos demonstrar que, dada uma álgebra $S$ com base $\mathcal{D}$ que admita uma teoria de Bases de Gröbner e um ideal $J$ de $S$, a projeção de $\mathcal{D}$ sobre $S / J$ (excetuando-se o zero) será uma base multiplicativa se e somente se $J$ for um ideal 2-nomial (Proposição 3.4.22).

A próxima pergunta natural seria se a imagem de $\mathcal{D}$, nessas condições, também admite uma ordem admissível. A resposta para esta pergunta, infelizmente é negativa em geral e, até o momento em que esta dissertação foi escrita, os autores não conhecem nenhum resultado que mostre quais as condições necessárias para que a projeção de $\mathcal{D}$ admita alguma ordem admissível [Gre00, Oli02].

Em seguida, exploraremos com maior cuidado esses comentários, uma vez que eles estão diretamente ligados com o Teorema 3.4.11. Feito isso, apresentaremos alguns resultados de cunho mais técnico e finalmente a demonstração do teorema acima.

No que segue, sejam $R, \mathcal{B}, \leq$ e $I$ como no enunciado do Teorema 3.4.11.

\subsubsection{Congruências}

Considere $\mathcal{C}$ o conjunto de todas as congruencias em $\mathcal{B} \cup\{0\}$ e $\mathcal{I}$ o conjunto de todos os ideais 2-nomiais de $R$. Definimos a aplicação $\varphi: \mathcal{C} \longrightarrow \mathcal{I}$ que associa uma congruència $c \in \mathcal{C}$ ao ideal $\hat{\mathcal{P}}(c):=\langle\{(a-b) \mid(a, b) \in c\}\rangle$. Note que como $c$ é uma congruência em $\mathcal{B} \cup\{0\}$, segue que $\gamma(c)$ é um ideal 2-nomial, para todo $c \mathrm{em} \mathcal{C}$.

Iniciamos com algumas questões técnicas necessárias para a Proposição 3.4.18. Em seguida, esta proposição será utilizada na demonstração de que existe uma bijeção entre $\mathcal{C}$ e $\mathcal{I}$ (no Lema 3.4.19). O principal objetivo desta subseção é utilizar esse maquinário para demonstrar a Proposição 3.4.22.

\footnotetext{
${ }^{8}$. Tote que as condiçōes 1 e 2 do teorema são equivalentes a exigir que $\mathcal{B}=\phi(\mathcal{P}) \backslash\{0\}$.
} 
Primeiramente definiremos o conceito de expressão linear para um elemento de $R$ e exploraremos algumas de suas propriedades. Ressaltamos que estas observações serão utilizadas apenas na demonstração da Proposição 3.4 .18 e podem ser deixadas de lado pelo leitor não interessado nesta prova sem qualquer prejuízo para o restante do texto.

Uma expressão linear para um elemento $f$ de $R$ (com respeito a $\mathcal{B}$ ) é uma expressão da forma: ${ }^{9}$

$$
e=\sum_{i \in H} \alpha_{i} b_{i}
$$

em que $H$ é um conjunto (finito) de índices e, para todos os valores $i$ de $H, \alpha_{i}$ é um escalar não nulo e $b_{i}$ é um elemento de $\mathcal{B}$. Ademais, $e=f$.

Note que não assumimos que $b_{h} \neq b_{j}$, se $h \neq j$. Com isso, podemos ter mais de uma expressão para um mesmo elemento $f$, apesar de $\mathcal{B}$ ser uma base de $R$. Diremos ainda que $\operatorname{card}(H)$ é o comprimento da expressão $e$ e o denotaremos por $\|e\|$.

Definimos ainda o suporte da expressão $e$ como $\operatorname{supp}(e):=\left\{b_{i} \mid i \in H\right\}$ e, para cada monômio $b$, a família de coeficientes de $b$ em $e$ como sendo $\operatorname{coef}_{e}(b):=\left(\alpha_{i} \mid b_{i}=b\right.$, para $\left.i \in H\right)$.

Dizemos que duas expressões $e$ e $e^{\prime}$ são iguais se $\operatorname{coef}_{e}(b)=\operatorname{coef}_{e^{\prime}}(b)$, para todo $b$ em $\mathcal{B}$ e que $e$ e $e^{\prime}$ são equivalentes se $e=e^{\prime}$ em $R$, isto é, se ambas representam o mesmo elemento.

Dada uma expressão linear $e$ para um elemento $f$, diremos que $e$ é uma expressão linear reduzida para $f$ quando $e=\sum_{i \in H} \alpha_{i} b_{i}$ satisfaz $b_{h} \neq b_{j}$, se $h \neq j$. Em outras palavras, uma expressão linear reduzida é simplesmente uma combinação linear de elementos de $\mathcal{B}$ em que não aparecem coeficientes nulos (e nem termos repetidos). Assim, é imediato ver que, dado um elemento $f$, existe uma única expressão linear reduzida para $f$.

Seja $e=\sum_{i \in H} \alpha_{i} b_{i}$ uma expressão linear para um elemento $f$ de $R$. Assuma ainda que $b_{h}=b_{j}$, para alguns índices $h$ e $j$ de $H$. Nestas condições, uma simplificação de e é uma expressão linear da forma

$$
e^{\prime}=\sum_{i \in H^{\prime}}\left(\gamma_{i} b_{i}\right)
$$

de modo que $H^{\prime}=H \backslash\{j\}, \gamma_{i}=\alpha_{i}$, para todo $i$ em $H^{\prime}$ diferente de $h$ e $\gamma_{h}=\left(\alpha_{j}+\alpha_{h}\right)$ (no caso em que $\gamma_{h}=0$, eliminamos $h$ de $H^{\prime}$ ). Os índices $h$ e $j$ são chamados de índices de simplificação.

Note que o comprimento de $e^{\prime}$ é estritamente menor que o comprimento de $e$ (na realidade, não é difícil ver que $\left\|e^{\prime}\right\|=\|e\|-1$ ou $\left.\left\|e^{\prime}\right\|=\|e\|-2\right)$. Ainda, é claro que $e^{\prime}$ é equivalente a $e$.

Uma seqüência de simplificações a partir de uma expressão linear $e_{0}$ é uma seqüência da forma $\left(e_{0}, e_{1}, \ldots\right)$, de forma que $e_{i}$ é uma simplificação de $e_{i-1}$, para todo $i>0$.

Afirmação 3.4.12 Seja e uma expressão linear para um elemento $f$ de $R$. Então não existem seqüências infinitas de simplificações a partir de e.

Ademais, para toda seqüência de simplificações $\left(e, e_{1}, e_{2} \ldots, e_{n}\right)$, existe uma seqüência de simplificações (possivelmente vazia) $\left(e_{n+1}, e_{n+2}, \ldots, e_{r}\right)$ tal que $e_{r}$ é a expressão linear reduzida para $f$.

Demonstração. A primeira parte segue imediatamente do fato de que $\left\|e_{i}\right\|<\left\|e_{i-1}\right\|$, para todo $i>0$.

\footnotetext{
${ }^{9}$ Ctilizaremos simplesmente o termo "expressão" para denotar expressões lineares, quando o contexto tornar claro que estamos nos referindo ao tipo de expressão aqui definido.
} 
A segunda parte segue por um argumento indutivo. Seja $e_{n}=\sum_{i \in H} \alpha_{i} b_{i}$. Se não existem índices $h$ e $j$ em $H$ tais que $b_{h}=b_{j}$, então $e_{n}$ é uma expressão linear reduzida para $f$ e não há mais o que mostrar.

Senão, tome $e_{n+1}$ a simplificação de $e_{n}$ utilizando $h$ e $j$ como índices de simplificação.

$O$ resultado segue então da primeira parte e da unicidade da expressão linear reduzida para $f$.

A seguinte afirmação segue imediatamente das definições de expressão linear e de simplificação.

Afirmação 3.4.13 Sejam $f$ e $f^{\prime}$ elementos de $R$ e $x$ e $x^{\prime}$ expressões lineares para $f$ e $f^{\prime}$, respectivamente.

Então $x+x^{\prime}$ é uma expressão linear para $f+f^{\prime}$. Conseqüentemente, uma simplificação $\bar{x}$ de $x+x^{\prime}$ é uma expressão linear para $f+f^{\prime}$. 3.4.18.

Em seguida apresentamos alguns lemas que serão fundamentais na demonstração da Proposição

Lema 3.4.14 Considere a álgebra de caminhos $K \Gamma$ com a base multiplicativa dada pelos caminhos em $\Gamma, \mathcal{P}$. Sejam $b_{1}, b_{1}^{\prime}, b_{2}, b_{2}^{\prime}, \ldots b_{k}, b_{k}^{\prime}$ elementos de $\mathcal{P}$ (não necessariamente distintos) $e \lambda_{1}, \ldots, \lambda_{k}$ coeficientes. Seja ainda

$$
f=\sum_{i=1}^{k} \lambda_{i}\left(b_{i}-b_{i}^{\prime}\right)
$$

Então $f$ não pertence a $\mathcal{P}$.

Demonstração. Tome a aplicação linear $\varphi: K \Gamma \longrightarrow K$ definida nos elementos da base $\mathcal{P}$ por: $\varphi(b)=1$, para todo $b \in \mathcal{P}$. Com isso, $\varphi$ é uma aplicação $K$-linear.

Assim, suponha que $f$ pertence a $\mathcal{P}$. Por um lado, $\varphi(f)=1$, pela definição de $\varphi$. Entretanto, por outro lado,

$$
\varphi(f)=\sum_{i=1}^{k} \lambda_{i}\left(\varphi\left(b_{i}\right)-\varphi\left(b_{i}^{\prime}\right)\right)=0
$$

e chegamos a uma contradição com a hipótese de $f$ pertencer a $\mathcal{P}$.

O lema a seguir é conseqüència imediata do resultado que acabamos de mostrar.

Lema 3.4.15 Sejam $b_{1}, b_{1}^{\prime}, b_{2}, b_{2}^{\prime}, \ldots b_{k}, b_{k}^{\prime}$ elementos de $\mathcal{P}$ (não necessariamente distintos) $e$ $\lambda_{1}, \ldots, \lambda_{k}$ coeficientes. Seja ainda

$$
f=\sum_{i=1}^{k} \lambda_{i}\left(b_{i}-b_{i}^{\prime}\right)
$$

e seja e uma expressão linear para $f$ com respeito a $\mathcal{P}$. Então, $\operatorname{card}(\operatorname{supp}(e)) \neq 1$. 
No que segue, assuma que temos uma congruência $c$ de $\mathcal{C}$ fixada.

Apresentamos em seguida a Propriedade $P$ para uma expressão linear da forma $e=\sum_{i \in H} \alpha_{i} b_{i}$. Esta propriedade será melhor explorada no Lema 3.4.16 e será fundamental para a demonstração da Proposição 3.4.18.

Propriedade $\mathbf{P}$ Existe um subconjunto $X$ de $H$ (denote ainda $Y=H \backslash X$ ) e uma partição $Q=\left\{Q_{1}, Q_{2}, \ldots, Q_{r}\right\}$ de $X$, tais que:

1. Para todo $j \in Y, b_{j} \sim_{c} 0$.

2. Para todo $1 \leq z \leq r$, se $x, y \in Q_{z}$, então $b_{x} \sim_{c} b_{y}$.

3. Para todo $1 \leq z \leq r, \sum_{i \in Q_{z}} \alpha_{i} b_{i}$ é uma expressão linear para um elemento da forma $f=\sum_{i=1}^{k} \lambda_{i}\left(b_{i}-b_{i}^{\prime}\right)\left(\lambda_{i}\right.$ são coeficientes e $b_{i}, b_{i}^{\prime}$ são monômios, para todo i).

Note o leitor que uma expressão $e$ que satisfaça a Propriedade $P$ pode ser vista como $e=r_{1}+r_{2}$, onde $r_{1}:=\sum_{i \in X} \alpha_{i} b_{i}$ e $r_{2}:=\sum_{j \in Y} \alpha_{j} b_{j}$.

Lema 3.4.16 Seja $e=\sum_{i \in H} \alpha_{i} b_{i}$ uma expressão linear que satisfaz a Propriedade $\mathrm{P}$ e $e^{\prime}=$ $\sum_{i \in H^{\prime}} \gamma_{i} b_{i}$ uma simplificação de e. Então é satisfaz a Propriedade P.

Demonstração. Suponha que os índices de simplificação são $h$ e $j$. Seja ainda $X$ um subconjunto de $H$ e $Q=\left\{Q_{1}, Q_{2}, \ldots, Q_{r}\right\}$ uma partição de $X$ nas condições da Propriedade $P$.

Seguiremos com um estudo de casos. Para cada caso, encontraremos um subconjunto $X^{\prime}$ de $H^{\prime}$ e uma partição $Q^{\prime}$ de $X^{\prime}$, que mostram que $e^{\prime}$ satisfaz a Propriedade $P$.

Temos então três possibilidades:

- Ambos $h$ e $j$ pertencem a $X$.

Neste caso, como $e$ satisfaz a Propriedade $P$, existem índices $1 \leq t, w \leq r$ (não necessariamente distintos) de forma que $h$ pertence a $Q_{t}$ e $j$ pertence a $Q_{w}$.

Logo, como $b_{h}=b_{j}$, segue que todos os monômios com índices em $Q_{t} \cup Q_{w}$ são equivalentes por $c$. Em outras palavras, se $x_{1}$ e $x_{2}$ pertencem a $Q_{t} \cup Q_{w}$, então $b_{x_{1}} \sim_{c} b_{x_{2}}$.

Temos ainda dois subcasos:

- A soma $\alpha_{h}+\alpha_{j}$ é zero.

Então $H^{\prime}=H \backslash\{h, j\}$. Tomemos $X^{\prime}=X \backslash\{h, j\}, A=\left(Q_{t} \cup Q_{w}\right) \backslash\{h, j\} \mathrm{e}$ $Q^{\prime}=\left(Q \backslash\left\{Q_{t}, Q_{w}\right\}\right) \cup\{A\}$.

Então, a condição 1 da Propriedade $P$ é facilmente verificada. A condição 2 segue dos argumentos dados acima.

Finalmente, a condição 3 segue do fato de $\sum_{i \in A} \alpha_{i} b_{i}$ ser uma simplificação para $\sum_{i \in Q_{t}} \alpha_{i} b_{i}$, se $t=w$, ou para $\sum_{i \in Q_{t}} \alpha_{i} b_{i}+\sum_{i \in Q_{w}} \alpha_{i} b_{i}$, se $t \neq w$ e da Afirmação 3.4.13.

- A soma $\alpha_{h}+\alpha_{j}$ é diferente de zero.

Neste caso, $H^{\prime}=H \backslash\{j\}$. Como antes, existem índices $1 \leq t, w \leq r$ (não necessariamente distintos) de forma que $h$ pertence a $Q_{t}$ e $j$ pertence a $Q_{w}$. 
O resultado segue de forma análoga à anterior, tomando $X^{\prime}=X \backslash\{j\}, A=$ $\left(Q_{t} \cup Q_{w}\right) \backslash\{j\}$ e $Q^{\prime}=\left(Q \backslash\left\{Q_{t}, Q_{w}\right\}\right) \cup\{A\}$.

- Ambos $h$ e $j$ pertencem a $Y$.

É fácil ver que $e^{\prime}$ satisfaz a Propriedade $P$ nesta situação se tomarmos $X^{\prime}=X$ e $Q^{\prime}=Q$.

- O índice $h$ pertence a $X$ e $j$ pertence a $Y{ }^{10}$

Neste caso, existe $1 \leq t \leq r$, de modo que $h$ pertence a $Q_{t}$. Assim, é fácil ver que $e^{\prime}$ satisfaz a Propriedade $P$ tomando $X^{\prime}=X \backslash Q_{t}$ e $Q^{\prime}=Q \backslash\left\{Q_{t}\right\}$.

O lema a seguir é conseqüência imediata da condição 3 da Propriedade $P$ e do Lema 3.4.15.

Lema 3.4.17 Seja e uma expressão linear satisfazendo a Propriedade P. Então, $\operatorname{card}\left(Q_{z}\right) \neq 1$, para todo $1 \leq z \leq r$.

Apresentamos então a Proposição 3.4.18. Ela será fundamental para os demais resultados que relacionam ideais 2-nomiais e congruências em $\mathcal{B} \cup\{0\}$ e sua demonstração faz uso dos comentários que fizemos acerca de expressões lineares.

Proposição 3.4.18 Seja $J$ um ideal 2-nomial de $R$ (e, portanto, um elemento de I) e c uma congruência em $\mathcal{B} \cup\{0\}$ (e, portanto, um elemento de $\mathcal{C}$ ). Suponha ainda que $\varphi(c)=J$. Nestas condições, as seguintes afirmações são verdadeiras.

1. Se a é um elemento de $\mathcal{B}$, tal que $a \in J$, então $(a, 0) \in c$.

2. Se a e b são elementos de $\mathcal{B}$, tais que $a-b \in J$, então $(a, b) \in c$.

Demonstração. Naturalmente, como $J=\varphi(c)$, segue pela definição da aplicação $\varphi$, que $J$ é o ideal 2-nomial gerado pelo conjunto

$$
A:=\left\{g_{1}-g_{2}, g_{3}-g_{4}, \ldots, f_{1}, f_{2}, \ldots\right\}
$$

de forma que, para todo $h \geq 1$, os elementos $g_{h}$ e $f_{h}$ pertencem a $\mathcal{B}$ e todos os pares ordenados $\left(g_{h}, g_{h+1}\right)$ e $\left(f_{h}, 0\right)$ pertencem à congruência $c$.

Com isso, todo elemento $f$ de $J$ é gerado por elementos de $A$, o que nos leva a considerar expressões da forma $r_{1}+r_{2}$, onde:

$$
r_{1}=\sum_{i=1}^{n} \sum_{j=1}^{m_{l}}\left(\alpha_{i, j} u_{i, j} g_{h_{i}} v_{i, j}-\alpha_{i, j} u_{i, j} g_{h_{2}+1} v_{i, j}\right) \text { e } r_{2}=\sum_{l=1}^{m} \sum_{k=1}^{m_{l}}\left(\beta_{l . k} p_{l, k} f_{h_{l}} q_{l . k}\right)
$$

em que cada $\alpha_{t, r}$ e cada $\beta_{t, r}$ é um escalar não nulo, para todos t.r, e os elementos $u_{t, r}$, $v_{t, r}, p_{t, r}$ e $q_{t, r}$ são elementos de $\mathcal{B}$, para todos $t, r$.

\footnotetext{
${ }^{10}$ É claro que a situação em que $h$ pertence a $Y$ e $j$ a $X$ é absolutamente análoga.
} 
Lembramos que, como $c$ é uma congruência, equivalências entre elementos de $\mathcal{B} \cup\{0\}$ são preservadas sob multiplicação. Com isso, temos que os pares ordenados das formas

$$
\left(u_{i, j} g_{h_{i}} v_{i, j}, u_{i, j} g_{h_{i}+1} v_{i, j}\right) \text { ou }\left(u_{l, k} f_{h_{l}} v_{l, k}, 0\right)
$$

pertencem a $c$.

Vejamos que a expressão da Equação 3.2 pode ser modificada de forma a nos fornecer uma expressão linear para $f$ que satisfaça a Propriedade $P$.

Com efeito, elementos das formas $u_{i, j} g_{h_{i}} v_{i, j}$ ou $u_{l, k} f_{h_{l}} v_{l, k}$ podem ser nulos. Construímos então uma expressão linear $e$ para $f$, a partir da Equação 3.2, da seguinte forma:

- Removemos todo termo nulo da forma $u_{l, k} f_{h_{l}} v_{l, k}$ de $r_{2}$.

- Se houver um termo nulo da forma $u_{i, j} g_{h_{i}} v_{i, j}$ (resp. $u_{i, j} g_{h_{i+1}} v_{i, j}$ ) em $r_{1}$, removemolo e movemos $u_{i, j} g_{h_{i+1}} v_{i, j}$ (resp. $u_{i, j} g_{h_{i}} v_{i, j}$ ) para $r_{2}$. Caso, ambos sejam nulos, removemo-los de $r_{1}$.

- Reordenamos os termos dessa expressão e tomamos como $X$ o conjunto dos índices dos termos que aparecem em $r_{1}$. Tomamos como $Q$ a partição de $X$ que agrupa em cada parte elementos da forma $u_{i, j} g_{h_{i}} v_{i, j}$ e $u_{i, j} g_{h_{i+1}} v_{i, j}$.

Com isso, é imediato verificar que $e$ satisfaz a Propriedade $P$.

Portanto, podemos usar a Afirmação 3.4.12 e o Lema 3.4.16 para ver que todo elemento de $f$ pode ser representado por uma combinação linear de elementos de $\mathcal{P}$ (uma expressão linear reduzida) que satisfaz a Propriedade $P$.

Assim, seja $a$ um monômio em $J$. Claro que a única expressão linear reduzida para esse elemento é $a$. Pelos argumentos acima, vemos que a expressão $a$ satisfaz a Propriedade $P$. Segue então do Lema 3.4.17 e da Propriedade $P$ que $a \sim_{c} 0$.

Ainda, seja $a-b$ uma diferença de monômios em $J$. Se $a-b=0$, é claro que $a=b$, donde $(a, b) \in c$ por se tratar de uma relação de equivalência. Assumimos então que $a-b$ é não nulo.

Novamente, é imediato ver que $a-b$ é a única expressão linear reduzida para esse elemento. Pelo Lema 3.4.17, temos que ou ambos $a$ e $b$ são equivalentes a zero (donde segue que $a \sim_{c} b$ ) ou ambos pertencem a uma mesma parte $Q_{z}$, donde concluímos que $a \sim{ }_{c} b$.

Lema 3.4.19 A aplicação p é bijetora.

Demonstração. Para ver que $\rho$ é sobrejetora, tomemos um ideal 2-nomial $J$ de $R$. Então, existe um conjunto gerador de $J$ da forma $\left\{g_{1}-g_{2}, g_{3}-g_{1}, \ldots, f_{1}, f_{2}, \ldots\right\}$, onde cada $g_{i}$ e cada $f_{i}$ pertencem a $\mathcal{B}$, para todo $i \geq 1$.

Tomemos então o seguinte subconjunto de $(\mathcal{B} \cup\{0\}) \times(\mathcal{B} \cup\{0\})$ :

$$
C:=\left\{\left(g_{1}, g_{2}\right),\left(g_{3}, g_{4}\right), \ldots,\left(f_{1}, 0\right),\left(f_{2}, 0\right), \ldots\right\}
$$


Denotemos por $r$ a relação de equivalência gerada por $C$. Então

$$
r=\left(C \cup C^{-1}\right)^{(\infty)}=\bigcup_{i=1}^{\infty}\left(C \cup C^{-1}\right)
$$

Mas, pelas propriedades de fechamento de ideais, não é difícil ver que $a-b$ pertence a $J$, para todo $(a, b)$ em $C^{-1}$. Ademais, um argumento indutivo em $i$ (juntamente com as propriedades de fechamento de $J$ ) nos mostram que $a-b$ pertence a $J$, para todo $(a, b)$ em $r$.

Finalmente, se tomamos $c$ o fecho multiplicativo de $r$, teremos que $c$ é a congruência gerada por $C$ e é fácil ver que $\varphi(c)$ está contido em $J$. Por outro lado, $\varphi(c)$ contém todos os geradores de $J$ (e é um ideal, pela definição de $\varphi$ ), donde concluímos que $\varphi(c)=J$.

Para ver que $\varphi$ é injetora, tome duas congruências $c_{1}$ e $c_{2}$, tais que $\varphi\left(c_{1}\right)=\varphi\left(c_{2}\right)$. Então, pelo Lema 3.4 .18 e pela definição da aplicação $\varphi$, temos que

$$
a \sim_{c_{1}} b \Leftrightarrow a-b \in \varphi\left(c_{1}\right) \Leftrightarrow a-b \in \varphi\left(c_{2}\right) \Leftrightarrow a \sim_{c_{2}} b
$$

e segue que $c_{1}=c_{2}$.

Em seguida apresentamos dois lemas bastante técnicos, mas que serão muito importantes na demonstração da Proposição 3.4.22.

Lema 3.4.20 Seja $x$ um elemento de $R$ tal que

$$
x=\sum_{i=1}^{m} \alpha_{i} b_{i}=\sum_{j=1}^{l} \beta_{j} a_{j}
$$

com $\alpha_{i}$ e $\beta_{j}$ em $K$ e $b_{i}$ e $a_{j}$ monômios, para $1 \leq i \leq m e 1 \leq j \leq l$. Assuma também que os coeficientes são todos não nulos e que os elementos $b_{i}$ são dois a dois distintos. Seja ainda c uma congruência em $\mathcal{C}$ e fixe uma classe qualquer de $c$, [b]. Então,

$$
\sum_{b_{i} \in[b]} \alpha_{i} b_{i}=\sum_{a_{j} \in[b]} \beta_{j} a_{j}
$$

para $1 \leq i \leq m$ e $1 \leq j \leq l$.

Demonstração. Como os elementos $b_{i}$ estão todos agrupados, possuem coeficientes não nulos e são todos elementos de uma base de $R$, temos que o suporte de $x$ é formado exatamente pelos elementos $b_{i}$. Com isso, para cada valor de $i$, existe um valor $l(i) \mathrm{e}$ índices $i_{1}, i_{2}, \ldots, i_{l(i)}$ tais que $a_{i_{1}}=a_{i_{2}}=\cdots=a_{i_{l(i)}}=b_{i}$. Ademais, podem existir elementos $a_{j}$ que não fazem parte do suporte de $x$. Para formalizar esse fato, tomamos um inteiro $p$ e inteiros $t(1), t(2), \ldots t(p)$ tais que $a_{k_{1}}=a_{k_{2}}=\cdots=a_{k_{t(k)}} \notin \operatorname{supp}(x)$, para todo $1 \leq k \leq p$. Com essa notação, podemos reescrever a soma do enunciado da seguinte maneira: 


$$
\sum_{i=1}^{m} \alpha_{i} b_{i}=\sum_{j=1}^{l} \beta_{j} a_{j}=\sum_{i=1}^{m}\left(\beta_{i_{1}}+\beta_{i_{2}}+\cdots \beta_{i_{l(i)}}\right) b_{i}+\sum_{k=1}^{p}\left(\beta_{k_{1}}+\beta_{k_{2}}+\cdots+\beta_{k_{t(k)}}\right) a_{k_{1}}
$$

em que $\beta_{i_{1}}+\beta_{i_{2}}+\cdots \beta_{i_{l(i)}}=\alpha_{i}$, para todo $1 \leq i \leq m$ e $\beta_{k_{1}}+\beta_{k_{2}}+\cdots+\beta_{k_{t(k)}}=0$, para todo $1 \leq k \leq p$.

Considere então uma classe $[b]$ de $c$ e tome a soma $\sum_{b_{i} \in[b]} \alpha_{i} b_{i}$ (para $1 \leq i \leq m$ ), ou seja, somamos os elementos da intersecção entre o suporte de $x$ e a classe $[b]$ (multiplicados por seus respectivos coeficientes em $x$ ).

Pelas igualdades acima, vemos que, para cada $1 \leq i \leq m$, os elementos $a_{i_{1}}, a_{i_{2}}, \ldots, a_{i_{l(i)}}$ e $b_{i}$ estão todos na mesma classe de equivalência (eles são iguais). Pela mesma razão, os elementos $a_{k_{1}}, a_{k_{2}}, \ldots, a_{k_{t_{k}}}$ também estão na mesma classe de equivalência, para $1 \leq k \leq p$. Assim, podemos utilizar essa discussão e as igualdades entre os coeficientes mostradas acima para escrever

$$
\sum_{b_{i} \in[b]} \alpha_{i} b_{i}=\sum_{b_{i} \in[b]}\left(\beta_{i_{1}}+\beta_{i_{2}}+\cdots \beta_{i_{l(i)}}\right) b_{i}+\underbrace{\sum_{a_{k_{1}} \in[b]}\left(\beta_{k_{1}}+\beta_{k_{2}}+\cdots+\beta_{k_{t_{k}}}\right) a_{k_{1}}}_{=0}=\sum_{a_{j} \in[b]} \beta_{j} a_{j}
$$

para $1 \leq i \leq m$ e $1 \leq k \leq p$, o que encerra a demonstração.

Lema 3.4.21 Seja I um ideal 2-nomial de $R$ e consideremos a (única) congruência $c$ em $\mathcal{C}$ tal que $\varphi(c)=I$. Tomemos um elemento $x=\sum_{i=1}^{m} \alpha_{i} b_{i}$, com $\alpha_{i}$ escalares não nulos e cada $b_{i} \in \mathcal{B}$, para $1 \leq i \leq m$ (assumimos que os elementos dessa soma estão todos agrupados, ou seja, os elementos $b_{i}$ são dois a dois distintos, para $1 \leq i \leq m$ ). Nestas condições, $x$ pertence a $I$ se e somente se $\sum_{b_{i} \in[b]} \alpha_{i} b_{i}$ pertence a $I$, para cada classe de equivalência $[b]$ de $c$.

Demonstração. Como as classes [b] formam uma partição de $\mathcal{B} \cup\{0\}$ (por $c$ ser uma congruência), é claro que

$$
x=\sum_{[b] \in c} \sum_{b_{i} \in[b]} \alpha_{i} b_{i}, 1 \leq i \leq m
$$

de maneira que se cada soma da forma $\sum_{b_{i} \in[b]} \alpha_{i} b_{i}$ pertence a $I$, então $x$ também pertence a $I$. Vejamos agora a implicação reversa.

Suponha que $x$ pertence a $I$. Mas como $I$ é 2-nomial, podemos encontrar um conjunto gerador $G=\left\{g_{1}-g_{2}, g_{3}-g_{4}, \ldots, f_{1}, f_{2}, \ldots\right\}$, de forma que os elementos $g_{h}$ e $f_{h}$ são monòmios e tanto $\left(g_{h}, g_{h+1}\right)$ quanto $\left(f_{h}, 0\right)$ pertencem a $c$, para todo $h \geq 1$. Assim, como $x$ é um elemento de $I$, ele se escreve da seguinte maneira:

$$
x=\sum_{i=1}^{n} \sum_{j=1}^{n_{2}}\left(\alpha_{i, j} u_{i, j} g_{h_{i}} v_{i, j}-\alpha_{i, j} u_{i, j} g_{h_{i}+1} v_{i, j}\right)+\sum_{l=1}^{y} \sum_{k=1}^{y_{l}}\left(\alpha_{l . k} u_{l . k} f_{h_{l}} v_{l . k}\right)
$$


com cada $\alpha_{t, r}$ um escalar não nulo, para todos $t, r$, e $u_{t, r}, v_{t, r}$ elementos de $\mathcal{B}$, para todos $t, r$.

Vale notar que algumas das multiplicações acima podem ser zero. Assim, podemos reescrever $x$ como um elemento da forma:

$$
x=\sum_{i=1}^{m} \alpha_{i} b_{i}=\sum_{z=1}^{q_{1}} \gamma_{z}\left(a_{z_{1}}-a_{z_{2}}\right)+\sum_{d=1}^{q_{2}} \beta_{d} p_{d}
$$

de modo que as seguintes condições são satisfeitas, para todos $1 \leq z \leq q_{1}$ e $1 \leq d \leq q_{2}$ :

- $\gamma_{z}$ e $\beta_{d}$ são escalares não nulos.

- Cada $a_{z_{1}}$ (resp. $a_{z_{2}}$ ) é um múltiplo não nulo de $g_{h_{i}}$ (resp. $g_{h_{i}+1}$ ), para algum $i$.

- Cada $p_{d}$ é um múltiplo não nulo de $f_{h_{l}}$, para algum $l$, de $g_{h_{i}}$ ou de $g_{h_{i}+1}$, para algum $i$.

- Cada elemento da forma $\gamma_{z}\left(a_{z_{1}}-a_{z_{2}}\right)$ ou $\beta_{d} p_{d}$ pertence a $I$.

Note agora que $a_{z_{1}} \sim_{c} a_{z_{2}}$, para todo $z$. Assim, podemos usar o Lema 3.4 .20 para concluir que

$$
\sum_{b_{i} \in[b]} \alpha_{i} b_{i}=\sum_{i=1}^{o} \gamma_{z_{i}}\left(a_{z_{i_{1}}}-a_{z_{i_{2}}}\right)+\sum_{j=1}^{o^{\prime}} \beta_{d_{j}} p_{d_{j}}
$$

o que mostra que $\sum_{b_{i} \in[b]} \alpha_{i} b_{i}$ pertence a $I$.

Apresentamos em seguida o resultado prometido que associa o fato de a projeção de uma base multiplicativa ser multiplicativa com ideais 2-nomiais.

Proposição 3.4.22 Seja $S$ uma $K$-álgebra com uma base multiplicativa $\mathcal{D}$. Seja $J$ um ideal de $S$, $\pi: S \longrightarrow S / J$ o homomorfismo canônico e denote por $\mathcal{B}=\pi(\mathcal{D}) \backslash\{0\}$. Então $\mathcal{B}$ é uma base multiplicativa de $S / J$ se e somente se $J$ é um ideal 2-nomial.

Demonstração. Vejamos primeiramente que se $\mathcal{B}$ é uma base multiplicativa de $S / J$, então o ideal $J$ é 2-nomial.

Defina a seguinte congruência $c$ entre elementos de $\mathcal{D} \cup\{0\}$ : dados $d, d^{\prime}$ em $\mathcal{D}$, dizemos que $d \sim_{c} d^{\prime}$ se $\pi(d)=\pi\left(d^{\prime}\right)$. Não é difícil verificar que essa relação é de fato reflexiva, simétrica, transitiva e compatível com a multiplicação em $\mathcal{D}$. Pelo Lema 3.4.19, existe um único ideal 2-nomial $J_{c}$ tal que $\varphi(c)=J_{c}$. Mostraremos que $J=J_{c}$.

Para verificar que $J_{c} \subseteq J$, basta tomar um conjunto gerador de $J_{c}$ formado por elementos da forma $a-b$, com $a$ e $b$ em $\mathcal{D} \cup\{0\}$. Então: ${ }^{11}$

$$
a-b \in J_{c} \Leftrightarrow(a, b) \in c \Leftrightarrow \pi(a)=\pi(b) \Leftrightarrow a-b \in J
$$

\footnotetext{
${ }^{11}$ Algumas das implicações abaixo fazem uso do Lema 3.4.18.
} 
Para ver a inclusão contrária, considere a projeção canônica $\pi_{c}: S \longrightarrow S / J_{c}$ e tome $b \in J$. Precisamos mostrar que $\pi_{c}(b)=0$. Como $b$ é um elemento de $S$, ele se escreve de maneira única como combinação linear de elementos de $\mathcal{D}$. Seja então $b=\sum_{i=1}^{m} \alpha_{i} d_{i}$, com $d_{i} \in \mathcal{D}$ e $\alpha_{i}$ escalares não nulos, para todo $i$. Por outro lado, como $b$ é um elemento de $J$, temos que $\pi(b)=0$. Logo,

$$
\pi(b)=\sum_{i=1}^{m} \alpha_{i} \pi\left(d_{i}\right)=0
$$

Naturalmente, podemos ter alguns $d_{i}$ que possuem a mesma imagem por $\pi$ e, eventualmente, alguns deles terão imagem nula. Como $\mathcal{B}=\pi(\mathcal{D}) \backslash\{0\}$ é uma base multiplicativa de $S / J$, temos que, agrupando os coeficientes dos elementos $d_{i}$ de mesma imagem não nula, a soma de seus coeficientes deve ser zero. ${ }^{12}$

Mais formalmente, seja $i_{1}, i_{2}, \ldots, i_{k}$ um conjunto qualquer de índices (entre 1 e $n$ ) satisfazendo $\pi\left(d_{i_{1}}\right)=\pi\left(d_{i_{k}}\right)=\cdots \pi\left(d_{i_{k}}\right) \neq 0$. Então, $\alpha_{i_{1}}+\alpha_{i_{2}}+\cdots+\alpha_{i_{k}}=0$. Mas neste caso, teremos as seguintes implicações. ${ }^{13}$

$$
\begin{aligned}
& \pi\left(d_{i_{1}}\right)=\pi\left(d_{i_{k}}\right)=\cdots \pi\left(d_{i_{k}}\right) \\
& \quad \Leftrightarrow \quad d_{i_{1}} \sim_{c} d_{i_{2}} \sim_{c} \cdots \sim_{c} d_{i_{k}} \\
& \quad \Leftrightarrow \quad \pi_{c}\left(d_{i_{1}}\right)=\pi_{c}\left(d_{i_{2}}\right)=\cdots=\pi_{c}\left(d_{i_{k}}\right)
\end{aligned}
$$

Mas então, pela observação envolvendo os coeficientes desses elementos feita logo acima, teremos que $\pi_{c}\left(\alpha_{i_{1}} d_{i_{1}}+\alpha_{i_{2}} d_{i_{2}}+\cdots+\alpha_{i_{k}} d_{i_{k}}\right)=0$.

Por outro lado, se $i$ é um índice tal que $\pi\left(d_{i}\right)=0$, então $d_{i} \sim_{c} 0$, donde $\pi_{c}\left(d_{i}\right)=0$. Com isso concluímos que

$$
\pi_{c}(b)=\sum_{i=1}^{m} \alpha_{i} \pi_{c}\left(d_{i}\right)=0
$$

o que encerra essa parte da demonstração. Vejamos agora a implicação reversa, ou seja, que o fato de $J$ ser um ideal 2-nomial implica que $\mathcal{B}$ é uma base multiplicativa de $S / J$.

Conforme comentado anteriormente na demonstração, $\mathcal{B} \backslash\{0\}$ será multiplicativa se for uma base, de forma que basta nos dedicaremos à tarefa de demonstrar esse último fato. Vejamos primeiramente que $\mathcal{B}$ gera $S / J$ como espaço vetorial.

Tome um elemento $a+J$ de $S / J$. Então, como $\mathcal{D}$ é base de $S$, temos que $a=\sum_{i=1}^{m} \alpha_{i} d_{i}$, com $\alpha_{i}$ escalares e $d_{i}$ elementos de $\mathcal{D}$, para $1 \leq i \leq m$. Mas então, $a+J=\pi(a)=$ $\sum_{i=1}^{m} \alpha_{i} \pi\left(d_{i}\right)$, onde cada $\pi\left(d_{i}\right)$ é um elemento de $\mathcal{B} \cup\{0\}$. Naturalmente, podemos desconsiderar os elementos nulos dessa soma e obteremos uma combinação linear de

\footnotetext{
${ }^{12}$ Na realidade, basta que $\mathcal{B}$ seja uma base. Com efeito, se temos dois elementos de $\mathcal{B}$, $\pi\left(d_{1}\right)$ e $\pi\left(d_{2}\right)$, então $\pi\left(d_{1}\right) \pi\left(d_{2}\right)=\pi\left(d_{1} d_{2}\right) \in \mathcal{B} \cup\{0\}$ por $\mathcal{D}$ ser uma base multiplicativa, donde a imagem de $\mathcal{D}$ por $\pi$ (excetuando-se o zero) sempre será multiplicativa se for uma base.

${ }^{13}$ A volta da última implicação faz uso do Lema 3.4.18.
} 
elementos de $\mathcal{B}$ igual a $a+J$. Vejamos então que $\mathcal{B}$ é um conjunto linearmente independente.

Tome uma combinação linear de elementos de $\mathcal{B}$ da forma $0=\sum_{i=1}^{m} \alpha_{i} \pi\left(d_{i}\right)$, em que cada $\alpha_{i}$ é um escalar não nulo e os elementos $d_{i}$ pertencem a $\mathcal{D}$, para $1 \leq i \leq m$. Podemos assumir ainda, sem perda de generalidade, que os elementos $\pi\left(d_{i}\right)$ são dois a dois distintos. Precisamos então mostrar que cada $\alpha_{i}=0$. Tome $c$ a congruência associada a $J$, ou seja $c$ é a única congruência tal que $\varphi(c)=J$.

Mas então, como $\sum_{i=1}^{m} \alpha_{i} d_{i} \in J$ (sua imagem por $\pi$ é zero, por hipótese), podemos utilizar o Lema 3.4.21, para ver que, para cada classe de equivalência [b] de $c, \sum_{d_{i} \in[b]} \alpha_{i} d_{i}$ pertence a $J$. Por outro lado, cada $d_{i}$ da combinação linear acima está em uma classe de equivalência de $c$ diferente, pois estamos assumindo que os valores de $\pi\left(d_{i}\right)$ são dois a dois distintos, para todo $i$. Assim, se tivermos dois índices $j, k$ tais que $d_{j} \sim_{c} d_{k}$, teremos que $d_{j}-d_{k} \in J$ pela escolha de $c$, o que nos leva a concluir que tais índices não existem.

Com isso, $\alpha_{i} d_{i}$ pertence a $J$, para todo $1 \leq i \leq m$. Logo, se $\alpha_{i} \neq 0$, teremos que $\alpha_{i}^{-1} \alpha_{i} d_{i}=d_{i}$ é um elemento de $J$, o que é um absurdo, pois estamos assumindo que $\pi\left(d_{i}\right) \neq 0$. Portanto, $\alpha_{i}=0$, para $1 \leq i \leq m$ e $\mathcal{B}$ é linearmente independente.

Esse resultado encerra nossos comentários sobre ideais 2-nomiais por hora. Note que a afirmação do Teorema 3.4.11 feita sobre ideais 2-nomiais é conseqüência direta da Proposição 3.4.22.

Esse fato será relembrado na demonstração desse teorema adiante. Nos voltamos agora para algumas questões mais técnicas que visam tornar a demonstração do Teorema 3.4.11 mais limpa.

\subsubsection{Demonstração do Teorema 3.4.11}

Assumimos a notação do enunciado do Teorema 3.4.11 e definiremos uma aplicação $\phi$ de $K \Gamma$ em $R$. Para tanto, primeiro definimos uma aplicação $\phi_{0}: \mathcal{P} \longrightarrow R$ :

- Se $v$ é um elemento de $V(\Gamma)$ então $\phi_{0}(v):=v$, ou seja, a imagem de $v$ é o idempotente de $\mathcal{B}$ que deu origem ao vértice $v$ no grafo $\Gamma$.

- Se $e_{v_{1} v_{2}}$ é um elemento de $A(\Gamma)$ com ponta inicial $v_{1}$ e ponta final $v_{2}$, então $\phi_{0}\left(e_{v_{1} v_{2}}\right):=e$, onde $e$ é o elemento de $E$ de origem $v_{1}$ e término $v_{2}$ que deu origem ao arco $e_{v_{1} v_{2}}$.

- Se $c$ é um caminho em $\Gamma$ (em outras palavras, um elemento da base $\mathcal{P}$ de $K \Gamma$ ), então ele se escreve de maneira única como $c=e_{1} e_{2} \cdots e_{n}$, onde cada $e_{i}$ é um arco (trata-se de sua forma canônica). Então definimos $\phi_{0}(c):=\phi_{0}\left(e_{1}\right) \phi_{0}\left(e_{2}\right) \cdots \phi_{0}\left(e_{n}\right)$.

Tendo definido como a aplicação $\phi_{0}$ atua nos elementos da base de $K \Gamma$, definimos $\phi$ como sendo a única extensão $K$-linear de $\phi_{0}$.

Lema 3.4.23 A aplicação $\phi$ definida acima é um homomorfismo de $K$-álgebras tal que $\mathcal{B}=\phi(\mathcal{P}) \backslash$ $\{0\}$.

Demonstração. Vejamos primeiramente o fato de que $\phi(\mathcal{P}) \backslash\{0\}=\mathcal{B}$. Por um lado, tomemos um caminho $c \in \mathcal{P}$. Então, pela definição da aplicação $\phi$, se $c$ é um vértice, 
então $\phi(c) \in \mathcal{B}$. Senão, temos que $\phi(c)=\phi\left(c_{1}\right) \phi\left(c_{2}\right) \cdots \phi\left(c_{n}\right)$, onde os elementos $c_{i}$ são arcos de $\Gamma$. Logo, cada $\phi\left(c_{i}\right)$ é um elemento de $\mathcal{B}$, pela definição da aplicação $\phi$ e segue que $\phi(c)$ pertence a $\mathcal{B} \cup\{0\}$, pelo fato de $\mathcal{B}$ ser uma base multiplicativa.

Para ver a inclusão contrária, tomamos $b$ em $\mathcal{B}$. Se $b$ pertence a $V$, segue que $b$ pertence a $\phi(\mathcal{P}) \backslash\{0\}$ pela própria construção de $\Gamma$ e pela definição de $\phi$. Senão, pela Proposição $3.4 .8, b=b_{1} b_{2} \cdots b_{n}$, com $b_{i}$ em $E$, para todo $1 \leq i \leq n$. Note ainda que, como $b \neq 0$, segue que $\mathrm{t}\left(b_{i}\right)=\mathrm{o}\left(b_{i+1}\right)$, para todo $1 \leq i<n$.

Logo, pela construção de $\Gamma$ e definição de $\phi$, existem $\operatorname{arcos} e_{1}, e_{2}, \ldots e_{n}$ em $\Gamma$, tais que $\phi\left(e_{i}\right)=b_{i}$, para todo $1 \leq i \leq n$ e $e_{1} e_{2} \cdots e_{n}$ é um caminho em $\Gamma$. Logo, $e_{1} e_{2} \cdots e_{n}$ pertence a $\mathcal{P}$ (é não nulo) e $\phi\left(e_{1} e_{2} \cdots e_{n}\right)=\phi\left(e_{1}\right) \phi\left(e_{2}\right) \cdots \phi\left(e_{n}\right)=b_{1} b_{2} \cdots b_{n}=b$. Portanto, $\mathcal{B} \subseteq \phi(\mathcal{P}) \backslash\{0\}$.

Vejamos agora que se trata de um homomorfismo. A aplicação $\phi$ é $K$-linear por construção. Resta-nos mostrar que se $f$ e $f^{\prime}$ são dois elementos de $K \Gamma$, então $\phi\left(f f^{\prime}\right)=$ $\phi(f) \phi\left(f^{\prime}\right)$. Mas se escrevemos $f$ e $f^{\prime}$ como combinação linear de caminhos, teremos $f=\sum_{i=1}^{n} \alpha_{i} c_{i}$ e $f^{\prime}=\sum_{j=1}^{m} \beta_{j} d_{j}$, com $\alpha_{i}$ e $\beta_{j}$ escalares e $c_{i}$ e $d_{j}$ elementos da base $\mathcal{P}$, para $1 \leq i \leq n$ e $1 \leq j \leq m$. Logo, $\phi\left(f f^{\prime}\right)=\sum_{i=1}^{n} \sum_{j=1}^{m} \alpha_{i} \beta_{j} \phi\left(c_{i}\right) \phi\left(d_{j}\right)=\phi(f) \phi\left(f^{\prime}\right)$.

O lema anterior também poderia ter sido obtido de uma maneira mais teórica utilizando o fato de que $K \Gamma$ é uma álgebra tensorial. Contudo, preferimos apresentar aqui uma demonstração mais explícita.

Com esses resultados em mãos, podemos apresentar a demonstração do Teorema 3.4.11.

\section{Demonstração do Teorema 3.4.11.}

O fato de $\phi$ ser homomorfismo e as condições 1 e 2 do teorema foram demonstrados no Lema 3.4.23. Para verificar que esta aplicação é sobrejetora, basta notar que $\mathcal{B}$ é uma base contida em $\operatorname{Im}(\phi)$ e que $\phi$ é $K$-linear.

Resta-nos verificar a condição 3 . Para tanto, note que, $R \cong K \Gamma / \operatorname{Ker}(\phi)$, pelo primeiro teorema do homomorfismo (Proposição 1.2.1). Como $\mathcal{P}$ é uma base multiplicativa, estamos nas condições da Proposição 3.4.22, donde segue o resultado.

Para ver a condição 4, é claro que basta mostrar que a imagem de todo arco e todo vértice de $\Gamma$ pela aplicação $\phi$ é não nula. Mas esse fato segue imediatamente da construção de $\Gamma$.

Finalmente, a condição 5 segue imediatamente do Lema 3.4.10.

\subsubsection{Algumas Considerações}

Embora não tenhamos a pretensão de explorar neste trabalho as principais implicações e consequièncias do Teorema 3.4.11, apresentamos aqui alguns comentários e resultados adicionais que estão relacionados a ele e não se encontram presentes na literatura até onde é de conhecimento dos autores.

Assumimos ao longo de toda esta seção que temos uma álgebra $R$ com base multiplicativa $\mathcal{B}$ e com uma ordem admissível $\leq$ fixada em $\mathcal{B}$. Consideramos ainda $\Gamma$ o grafo associado a $\mathcal{B}$ e tomamos 
a álgebra de caminhos $K \Gamma$, a aplicação $\phi$ definida na Seção 3.4 .3 e o ideal $I:=\operatorname{Ker}(\phi)$. Então, $R \cong K \Gamma / I$, pelo Teorema 3.4.11.

Discutimos inicialmente algumas condições que nos permitem "levantar" a ordem $\leq$ para $\mathcal{P}$. Em seguida, faremos alguns comentários breves sobre como o teorema pode ser utilizado para reduzir uma instância do problema da pertinência em uma álgebra qualquer $R$ para uma instância do mesmo problema em $K \Gamma$.

\section{Ordens}

Uma pergunta de interesse é se podemos encontrar uma ordem admissível em $\mathcal{P}$ que seja "compativel" com a ordem $\leq$ fixada em $\mathcal{B}$. Mais precisamente, procuramos uma ordem admissível $\leq$ ' em $\mathcal{P}$, de modo que $\phi(p)<\phi(q)$ implica $p<^{\prime} q$, para todos $p$ e $q$ em $\mathcal{P}$.

Esta subseção se dedica a encontrar algumas situações em que esse levantamento da ordem pode ser feito de uma maneira bastante simples. Para tanto, introduzimos o conceito de quasi-domínio.

Definição 3.4.5 (Quasi-domínio) Dizemos que $R$ é um quasi-dominio quando $R$ possuir a seguinte propriedade: se $p q \neq 0$, então $\phi(p q) \neq 0$, para todos os caminhos $p$ e $q$ de $\mathcal{P}$.

Comentamos que, pela definição acima, é imediato ver que se $R$ é um domínio de integridade, então é um quasi-domínio.

A construção de $\Gamma$, as definições de $\phi$ e de quasi-domínio, a condição 4 do Teorema 3.4 .11 e um argumento indutivo bastante natural nos permitem concluir o seguinte resultado.

Afirmação 3.4.24 Se $R$ é um quasi-domínio, então $\phi(p) \neq 0$, para todo $p$ em $\mathcal{P}$.

Um ponto fundamental para nossa discussão é que, se $R$ é um quasi-domínio, então é possível encontrar uma ordem $\leq^{\prime}$ com as propriedades desejadas.

Suponha então que $R$ é uma álgebra nas hipóteses apresentadas no início da seção e definiremos uma relação $\leq^{\prime}$ em $\mathcal{P}$ da seguinte maneira. Ordene os vértices e os arcos de $\Gamma$ e tome a ordem deglex em $\mathcal{P}$ com essa ordenação (denote-a por $<_{d}$ ). Tome agora dois elementos $p$ e $q$ de $\mathcal{P}$. Então dizemos que $p<^{\prime} q$ se

$$
\begin{aligned}
& \phi(p)<\phi(q) \text { ou } \\
& \phi(p)=\phi(q) \text { e } p<_{d} q \text { ou } \\
& \phi(p) \text { e } \phi(q) \text { são incomparáveis e } p<_{d} q
\end{aligned}
$$

Proposição 3.4.25 Se $R$ é um quasi-domínio, a relação $\leq$ 'definida acima é uma ordem admissível.

Demonstração. Precisamos mostrar que $<$ é uma relação de ordem e as propriedades 1 a 4 descritas na Definição 3.1.1.

Para ver que $<$ 'é uma ordem, basta utilizar a Afirmação 3.4.2.4 para ver que o terceiro caso de sua definição nunca pode ocorrer. A partir dessa observação, a prova de que $<^{\prime}$ é reflexiva, antissimétrica e transitiva é simples e deixada a cargo do leitor.

O fato de que $\leq$ é uma boa ordem segue de uma maneira bastante natural do fato de $\leq$ e deglex serem boas ordens. 
Para a segunda propriedade, assuma $p<^{\prime} q$ e tome $r$ em $\mathcal{P}$, de modo que $r p$ e $r q$ são não nulos. Então, pela definição de $\leq^{\prime}, \phi(p) \leq \phi(q)$, donde

$$
\phi(r) \phi(p) \leq \phi(r) \phi(q)
$$

Mas, por $R$ ser um quasi-domínio, e pela Afirmação 3.4.24, os dois lados da desigualdade dada pela Equação 3.4 são não nulos. Temos, portanto, duas possibilidades:

1. $\phi(r p)=\phi(r q)$.

Suponha então que $\phi(p)<\phi(q)$. Logo, pelo fato de $\leq$ ser admissível, teremos que $\phi(r p)<\phi(r q)$, um absurdo.

Portanto, $\phi(p)=\phi(q)$. Assim, como $p<^{\prime} q$, segue que $p<_{d} q$. Com isso, $r p<_{d} r q$ $\mathrm{e}$, conseqüentemente, $r p<^{\prime} r q$, como queríamos.

2. $\phi(r p)<\phi(r q)$.

Neste caso, $r p<^{\prime} r p$ pela definição da ordem $\leq^{\prime}$.

Claro que a propriedade 3 segue por argumentos análogos aos apresentados acima, de modo que nos resta verificar a propriedade 4.

Para tanto, tome $p$ e $q$, tais que $p$ divide $q$. Então existem caminhos $u$ e $v$, com $q=u p v$. Mas então, $\phi(q)=\phi(u) \phi(p) \phi(v)$ e temos que $\phi(q) \leq \phi(p)$, donde segue o resultado.

O seguinte exemplo nos traz a má notícia de que a técnica usada anteriormente para definir $<^{\prime}$ pode produzir ordens em $\mathcal{P}$ que não sejam admissíveis, caso $R$ não seja um quasi-domínio (independente das ordens dadas nos arcos e vértices de $\Gamma$ ).

Exemplo 3.4.1 Considere o grafo $\Gamma$ dado na figura abaixo e a álgebra $K \Gamma$.

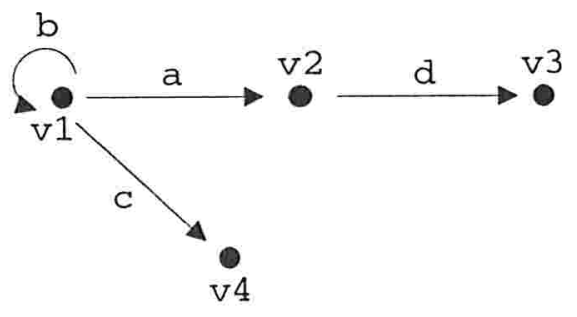

Seja ainda $I:=\left\langle\{a d-b c\} \cup J^{3}\right\rangle$ e tome $R=K \Gamma / I .^{14}$

Como o ideal $I$ è 2-nomial, a Proposição 3.4 .22 nos mostra que $\mathcal{B}:=\phi(\mathcal{P}) \backslash\{0\}$ é uma base multiplicativa para $R$. Assim, se considerarmos $R$ em conjunto com a base $\mathcal{B}$ como definidas acima, estaremos nas condições de nossa discussão anterior.

Defina então a seguinte ordem admissível $\leq \mathrm{em} \mathcal{B}:^{15}$

\footnotetext{
${ }^{14}$ Recordamos que $J$ é o ideal gerado pelos arcos de $\Gamma$.

${ }^{15}$ Como se trata de uma base finita, pode-se verificar que a ordem $\leq$ aqui apresentada é de fato admissível, por meio de uma análise de casos.
} 


$$
\phi(a)<\phi(b)<\phi(c)<\phi(d)<\phi(b a)<\phi\left(b^{2}\right)<\phi(b c)=\phi(a d)
$$

Com isso, dê uma ordem qualquer nos vértices e uma ordem qualquer nos arcos de $\Gamma$, desde que $a$ seja menor que $b$. Assim, $b a<^{\prime} a d$, pois $\phi(b a)<\phi(a d)$. Logo, se multiplicarmos os dois lados da desigualdade por $b$ à esquerda, teremos $b^{2} a<^{\prime} b a d$. Por outro lado, $\phi\left(b^{2} a\right)=\phi(b a d)=0$ e $b^{2} a>_{d} b a d$, donde concluímos que $b^{2} a>^{\prime} b a d$ pela definição de $\leq^{\prime}$, nos levando a uma contradição.

Tome agora uma ordem nos arcos em que $b$ é menor que $a$. Assim teremos que $a<^{\prime} b$, uma vez que $\phi(a)<\phi(b)$, donde concluímos que $a^{4}<b a^{3}$. Mas, novamente, $\phi\left(a^{4}\right)=$ $\phi\left(b a^{3}\right)=0$ e, pela definição da ordem $\leq^{\prime}$ o fato de que $b a^{3}<_{d} a^{4}$ implica $b a^{3}<^{\prime} a^{4}$, uma contradição com a hipótese de $\leq^{\prime}$ ser admissível.

Na realidade, o exemplo a seguir vai um passo além e nos mostra que, se $R$ não é um quasidomínio, então a relação $<^{\prime}$ pode não ser sequer uma relação de ordem em $\mathcal{P}$ (dependendo da ordem dada nos arcos).

Exemplo 3.4.2 Seja $X=\{x, y, z\}$ e $I:=\left\langle\{z\} \cup X^{2}\right\rangle$ um ideal de $K\langle X\rangle$. Se considera$\operatorname{mos} R=K\langle X\rangle / I$, então

$$
\mathcal{B}:=\phi\left(X^{*}\right) \backslash\{0\}=\{\phi(x), \phi(y)\}
$$

é uma base multiplicativa de $R$. Tomamos a seguinte ordem admissível em $\mathcal{B}$ :

$$
\phi(y)<\phi(x)
$$

Ainda, fixamos a ordem de grau e lexicografia em $X^{*} \operatorname{com} x<z<y$. Mas então, $\phi(y)<\phi(x)$ e concluímos que $y<^{\prime} x$. Ainda, $\phi(z)=0$ e concluímos, pela definição de $<^{\prime}$ que $x<^{\prime} z$. Logo, se $<^{\prime}$ fosse uma ordem, poderíamos concluir que $y<^{\prime} z$.

Contudo, $\phi(y)$ e $\phi(z)$ são incomparáveis e $y>_{d} z$, nos levando a concluir que $y>^{\prime} z$.

Nos voltamos em seguida para encontrar condições necessárias e suficientes para que $R$ seja um quasi-domínio. Além dessa caracterização, exploraremos também o caso particular em que $R$ é um domínio. Iniciamos com uma conseqüência do Lema 3.4.14.

Corolário 3.4.26 Se I é gerado somente por binômios puros e uniformes, então $\phi(p) \neq 0$, para todo $p$ em $\mathcal{P}$.

Demonstração. Claro que $\phi(p)=0$ se e somente se $p$ pertence a $I$.

Seja então $\mathcal{G}=\left\{b_{1}-b_{1}^{\prime}, b_{2}-b_{2}^{\prime}, \ldots\right\}$ um conjunto gerador para $I$ nas hipóteses do corolário e assuma por absurdo que $f$ pertence a $I$. Então ele é da forma:

$$
f=\sum_{i} \sum_{j} \lambda_{i, j}\left(u_{i, j} b_{h_{i}} v_{i, j}-u_{i, j} b_{h_{i}}^{\prime} v_{i, j}\right)
$$


Os elementos $\lambda_{i, j}$ são escalares não nulos e $u_{i, j}$ e $v_{i, j}$ são monômios.

Ainda, como os elementos $b_{i}-b_{i}^{\prime}$ são uniformes, podemos supor que cada monômio da soma acima é não nulo. O resultado segue então do Lema 3.4.14.

Proposição 3.4.27 A álgebra $R$ é um quasi-dominio se e somente se $I$ é gerado por binômios puros e uniformes.

Demonstração. Suponha que o ideal $I$ possui um conjunto gerador nas hipóteses da proposição e tome $p$ e $q$ em $\mathcal{P}$. Mas então $p q$ pertence a $\mathcal{P}$ se for não nulo e o resultado segue do Corolário 3.4.26.

Para ver a implicação reversa, assuma que $I$ não é gerado somente por binômios puros e uniformes e vejamos que $R$ não é um quasi-domínio.

Mas então podemos concluir que existe um monômio em $I$, pelo seguinte. Como $I$ é um ideal 2-nomial, segue que ele possui um conjunto gerador $\mathcal{G}$ composto somente por monômios e binômios puros. Diante de nossas hipóteses, temos que esse conjunto possui pelo menos um monômio ou possui um binômio puro que não é uniforme.

Portanto, no segundo caso, existe um binômio puro $a-b$ no conjunto $\mathcal{G}$ que não é uniforme. Assim, podemos tomar, sem perda de generalidade, dois monômios $u$ e $v$ de forma que $u a v \neq 0$ e $u b v=0$. Como $\mathcal{P}$ é uma base multiplicativa, segue que uav pertence a $\mathcal{B} \cap I$.

Com isso, acabamos de concluir que existe pelo menos um monômio $m$ em $I$. Ademais, pela condição 4 do Teorema 3.4.11, $I \subseteq J^{2}$, donde $m$ é um caminho de comprimento maior que um.

Logo, existem $p$ e $q$ em $\mathcal{P}$ tais que $m=p q$. Assim, $p q$ pertence a $\mathcal{P}$ (e, portanto, é não nulo), mas $\phi(p) \phi(q)=0$. Portanto, $R$ não é um quasi-domínio, como queríamos.

Em vista da condição 5 do Teorema 3.4.11, do Lema 3.4.14 e da Proposição 3.4.27, podemos apresentar o seguinte corolário.

Corolário 3.4.28 A álgebra $R$ é um quasi-domínio se e somente se o ideal I não contém monômios.

Nos voltamos agora para encontrar condições necessárias e suficientes para que $R$ seja um domínio de integridade.

Lema 3.4.29 A álgebra $R$ é um dominio se e somente se, para todos $b$ e $b^{\prime}$ em $\mathcal{B}, b b^{\prime} \neq 0$.

Demonstração. É claro que se $R$ é um domínio, então não existem $b$ e $b^{\prime}$ em $\mathcal{B}$ tais que $b b^{\prime}=0$.

Para demonstrar a implicação reversa, suponha que $R$ não é um domínio e mostraremos, por redução ao absurdo, que existem $b$ e $b^{\prime}$ em $\mathcal{B}$ satisfazendo $b b^{\prime}=0$. Assim, assuma que $R$ não é um domínio e que não existem $b$ e $b^{\prime}$ em $\mathcal{B}$ tais que $b b^{\prime}=0$.

Como $R$ não é um domínio, existem elementos não nulos $f$ e $g$ de $R$, tais que $f g=0$. Mas, como $\mathcal{B}$ é uma base de $R$, teremos: 


$$
\begin{aligned}
& f=\sum_{i=1}^{n} \alpha_{i} b_{i}, \text { com } 0 \neq \alpha_{i} \in K \text { e } b_{i} \in \mathcal{B}, \text { para todos } 1 \leq i \leq n \\
& g=\sum_{j=1}^{k} \beta_{j} c_{j}, \text { com } 0 \neq \beta_{j} \in K \text { e } c_{j} \in \mathcal{B}, \text { para todos } 1 \leq j \leq k
\end{aligned}
$$

Com isso,

$$
f g=\sum_{i=1}^{n} \sum_{j=1}^{k}\left(\alpha_{i} \beta_{j} b_{i} c_{j}\right)=0
$$

Note que $\alpha_{i} \beta_{j}$ é um elemento não nulo, para todos $i$ e $j$, uma vez que $K$ é um domínio de integridade. Ainda, cada $b_{i} c_{j} \neq 0$ por hipótese.

Assuma em seguida, sem perda de generalidade, que $b_{1}>b_{2}>\cdots>b_{n}$ e que $c_{1}>c_{2}>$ $\cdots>c_{k}$. Dessa forma, $\widehat{f}=b_{1}$ e $\widehat{g}=c_{1}$.

Afirmamos que $\hat{f} \widehat{g}=b_{1} c_{1}>b_{i} c_{j}$, para todos os valores de $i$ e de $j$ distintos de 1 . Para ver esta afirmação, tome $1 \leq h \leq k$. Então, $b_{1} c_{h}>b_{i} c_{h}$, para todo $1<i \leq n$, uma vez que $b_{1}>b_{i}$. Ainda, se fixamos um índice $1<h \leq k$, vemos que $c_{h-1}>c_{h}$, donde $b_{1} c_{h-1}>b_{1} c_{h}$. Logo, pelo argumento acima, $b_{1} c_{h-1}>b_{i} c_{h}$, para todo $1 \leq i \leq n$. Com isso $b_{1} c_{1}>b_{i} c_{j}$, para todos $1<i \leq n$ e $1<j \leq k$.

Assim, $b_{1} c_{1}$ aparece com coeficiente não nulo na Equação 3.5 e é estritamente maior que todos os outros monômios que aparecem nesta equação. Portanto, ele não é cancelado nesta soma e temos uma contradição com o fato de $f g$ ser igual a zero. Tal contradição encontra suas raízes na hipótese de os produtos da forma $b b^{\prime}$ serem não nulos, para todos $b$ e $b^{\prime}$ em $\mathcal{B}$, o que conclui a demonstração.

Com isso podemos apresentar condições necessárias e suficientes para que $R$ seja um domínio.

Proposição 3.4.30 A álgebra $R$ é um dominio de integridade se e somente se $K \Gamma$ contém apenas um vértice e I é puramente binomial.

Demonstração. Assuma que $R$ é um dominio de integridade. Vejamos primeiro que $K \Gamma$ contém apenas um vértice. Para tanto, suponha por absurdo que $v_{1}$ e $v_{2}$ são vértices distintos de $\Gamma$.

Pela definição da aplicação $\phi$ e pela construção de $\Gamma$, sabemos que $\phi\left(v_{1}\right)$ e $\phi\left(v_{2}\right)$ pertencem a $\mathcal{B}$ (e, portanto, são não nulos) e que são ortogonais. Assim, $\phi\left(v_{1}\right) \phi\left(v_{2}\right)=0$, o que contradiz o fato de $R$ ser um domínio.

Suponha agora por absurdo que $I$ não é um ideal puramente binomial. Lembramos que $I$ é 2-nomial pelo Teorema 3.4.11. Assim, $I$ possui um conjunto gerador composto somente por monômios e por binômios puros. Portanto, se assumimos que ele não é 
puramente binomial, podemos tomar um monômio $u$ em $I .{ }^{16}$ Pela construção de $\Gamma$ e pela definição de $\phi$, sabemos que a imagem de todo arco pela aplicação $\phi$ é não nula. Portanto, $u$ não é um arco de $\Gamma$. Com isso, $u=e_{1} e_{2} \cdots e_{n}$, com cada $e_{i}$ em $A(\Gamma)$. Assim, $0=\phi(u)=\phi\left(e_{1}\right) \phi\left(e_{2}\right) \cdots \phi\left(e_{n}\right)$ e os elementos $\phi\left(e_{1}\right), \phi\left(e_{2}\right) \ldots, \phi\left(e_{n}\right)$ são todos não nulos, levando a uma contradição com a hipótese de $R$ ser um domínio.

Vejamos em seguida a implicação reversa. Para tanto, assuma que $\Gamma$ contém apenas um vértice, que $I$ é puramente binomial e suponha por absurdo que $R$ não é um domínio. Vejamos que isso nos leva a uma contradição.

Mas como $R$ não é um domínio, o Lema 3.4 .29 e o Teorema 3.4.11 nos mostram que existem elementos $u$ e $p$ em $\mathcal{P}$, tais que $\phi(u) \phi(p)=\phi(u p)=0$.

Entretanto, como $\Gamma$ possui apenas um vértice, $\mathrm{o}(u)=\mathrm{o}(p)=\mathrm{t}(u)=\mathrm{t}(p)$, donde $u p \neq 0$.

Logo, up pertence a $I$. Ainda, como $\Gamma$ possui apenas um vértice, todos os elementos de $K \Gamma$ são uniformes. A contradição procurada segue então do Corolário 3.4.26.

Um comentário interessante acerca desta proposição é que se $R$ é um domínio, então a álgebra $K \Gamma$ é isomorfa a uma álgebra livre e é, portanto, indecomponível (veja a Seção 1.4).

Ademais, o Lema 3.4.29 e a Proposição 3.4 .30 nos mostram que se as multiplicações envolvendo elementos de $\mathcal{B}$ são não nulas (isto é, $\mathcal{B} \cdot \mathcal{B} \subseteq \mathcal{B}$ ), então $K \Gamma$ é isomorfa a uma álgebra livre.

\section{O Problema da Pertinência}

Mencionamos brevemente um uso bastante interessante do Teorema 3.4.11 que permite uma tentativa de aplicar a teoria vista até aqui (em especial os algoritmos que serão abordados no Capítulo 4) a álgebras arbitrárias (desde que elas possuam teoria de Bases de Gröbner).

Uma motivação para isso segue do fato de os algoritmos que serão apresentados neste trabalho terem seu foco nas álgebras de caminhos. Na realidade, os autores desconhecem quaisquer generalizações deles (especialmente no que concerne implementações desses algoritmos em pacotes computacionais) para casos mais gerais.

A grosso modo, os resultados do Teorema 3.4.11 fornecem a possibilidade de reduzir o problema de realizar cálculos em uma álgebra qualquer para cálculos em uma álgebra de caminhos.

Para ilustrar essa idéia, podemos supor que temos em mãos uma álgebra $R$, munida de uma base multiplicativa $\mathcal{B}$ com uma ordem admissível. Assumimos ainda que é conhecido o grafo associado a $\mathcal{B}$ (que denotaremos por $\Gamma$ ), de forma que $K \Gamma / I \cong R$. Note que o Lema 3.4.9 nos garante que, se $R$ for finitamente gerado, o grafo $\Gamma$ será finito.

Com isso, se temos um ideal $J$ de $R$ e um representante de classe $a$ para um elemento de $R$, podemos utilizar algoritmos em $K \Gamma$ para decidir a pertinência de $a+I$ em $J$. Claro que $a+I$ pertence a $J$ se e somente se a pertence ao ideal $\phi^{-1}(J)$ de $K \Gamma$. Lma pergunta natural seria então se (ou melhor, em que condições) $\phi^{-1}(J)$ possui uma Base de Gröbner finita.

Inicialmente conjeturamos que isso ocorreria se tanto $J$ quanto $I$ possuíssem Bases de Gröbner finitas. Contudo, até o presente momento, os autores não obtiveram nenhuma resposta definitiva para essa pergunta.

Por outro lado, sob a hipótese de $R / I$ ser de dimensão finita, $\phi^{-1}(J)$ possui Base de Gröbner finita. Isso ocorre pelo fato de que $\phi^{-1}(J)$ contém $I$. Assim, o fato de $R / I$ ser de dimensão finita implica que $R / \phi^{-1}(J)$ é de dimensão finita e podemos utilizar a Proposição 3.3.2.

\footnotetext{
${ }^{16}$ Na realidade, podemos assumir que $u$ pertence a um conjunto gerador de $I$, embora isto nāo seja necessário aqui.
} 


\section{Algoritmos e Outros Aspectos COMPUTACIONAIS}

Conforme comentado no Capítulo 2, a Teoria de Bases de Gröbner encontra grande parte de seu prestígio e importância no fato de ela exibir algoritmos (no caso não comutativo, seria mais preciso dizer procedimentos) para calcular formas normais e encontrar Bases de Gröbner. Nesse mesmo capítulo, foram apresentados o Algoritmo da Divisão para Polinômios em $K\left[x_{1}, \ldots, x_{n}\right]$ (Algoritmo 2.4.1) e o Algoritmo de Buchberger (Algoritmo 2.4.2). Nosso propósito aqui é fornecer uma introdução detalhada aos análogos deles para o caso não comutativo: o Algoritmo da Divisão e o Procedimento de Mora.

Com isso, a Seção 4.1 apresenta o Algoritmo da Divisão. Ela inicia abordando o contexto mais geral de qualquer álgebra que admita uma teoria de Bases de Gröbner. Nesse ponto, as principais propriedades do algoritmo são demonstradas. Ainda, a seção apresenta alguns comentários de implementação desse algoritmo para o caso das álgebras de caminhos.

As Seções 4.2 e 4.3 apresentam o Procedimento de Mora para as álgebras de caminhos. Na primeira, alguns aspectos algébricos necessários para o funcionamento do procedimento são explorados e esse é apresentado propriamente na seção seguinte. Apresentamos duas versões bastante presentes na literatura para o procedimento devidamente acompanhadas das demonstrações de suas correções.

Por fim, a Seção 4.4 fornece uma prova para a não computabilidade do problema de construir Bases de Gröbner no caso não comutativo.

\subsection{O Algoritmo da Divisão}

Esta seção tem o intuito de apresentar o Algoritmo da Divisão. Na Seção 2.4 foi vista uma descrição desse algoritmo para o caso comutativo e lá comentamos que sua generalização para o caso não comutativo praticamente não requer alterações. Por outro lado, naquela abordagem não tivemos o cuidado de explorar melhor as propriedades desse algoritmo, por brevidade. Nosso tratamento aqui será mais extenso.

A Subseção 4.1.1 apresentará uma descrição do algoritmo da divisão, à luz do que foi feito no Capítulo 2. Essa descrição será feita no contexto mais geral de uma álgebra qualquer que admita teoria de Bases de Gröbner. Neste ponto não estaremos preocupados com quaisquer questões computacionais relacionadas ao algoritmo, mas sim com as propriedades algébricas que ele possui. 
Em seguida, a Subseção 4.1.2 fornecerá alguns comentários sobre a implementação do Algoritmo da Divisão em álgebras de caminhos. Porém, prevenimos o leitor que não temos a pretensão de apresentar detalhes de implementação desse algoritmo nesse contexto (ou em qualquer outro), mas apenas de fornecer um panorama das principais questões relacionadas a ela.

\subsubsection{O Algoritmo da Divisão no Caso Geral}

Iniciamos com uma descrição do Algoritmo da Divisão (Algoritmo 4.1.1) e exploramos algumas de suas propriedades e sua relação com Bases de Gröbner. Essa descrição será feita em um contexto bastante amplo, de forma que não nos preocuparemos com a efetividade de todos os passos do algoritmo. Contudo, ressaltamos que os passos são todos efetivos no contexto das álgebras de caminhos, conforme será elaborado na seção seguinte. Comentamos ainda que a descrição dada aqui é não determinística.

Assim como feito até aqui, assuma que $R$ é uma álgebra finitamente gerada com base multiplicativa $\mathcal{B}$ e uma ordem admissível $\leq$ fixada em $\mathcal{B}$.

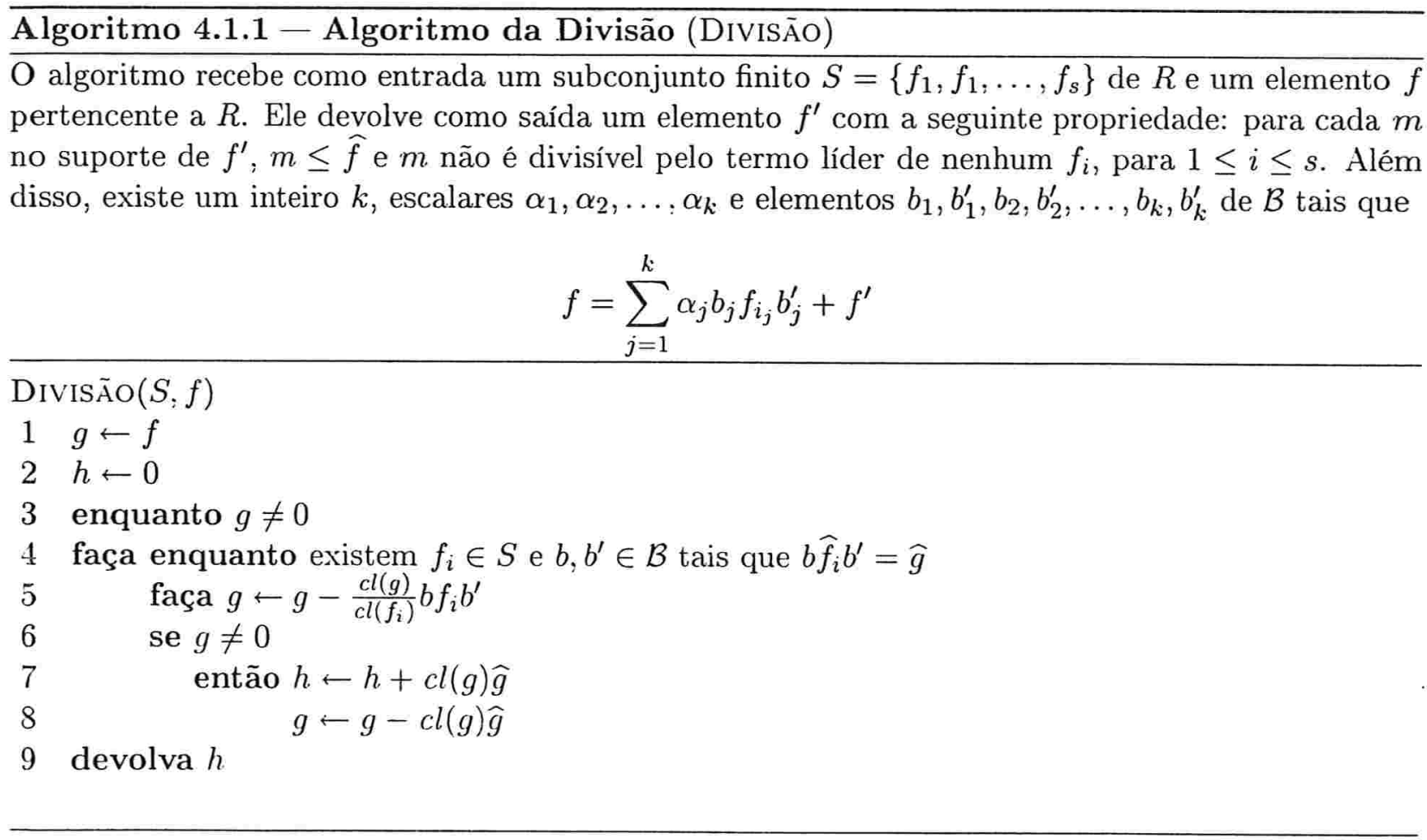

Na realidade, é fácil ver por sua descrição, que o Algoritmo da Divisão encontra os elementos $b_{i}, b_{i}^{\prime}($ com $1 \leq i \leq k)$, mas os omitimos de sua saída por simplicidade.

Apresentamos um exemplo de simulação do Algoritmo da Divisão para o caso das álgebras de caminhos.

Exemplo 4.1.1 Considere o grafo $\Gamma$ apresentado abaixo e a álgebra de caminhos $K \Gamma$. 


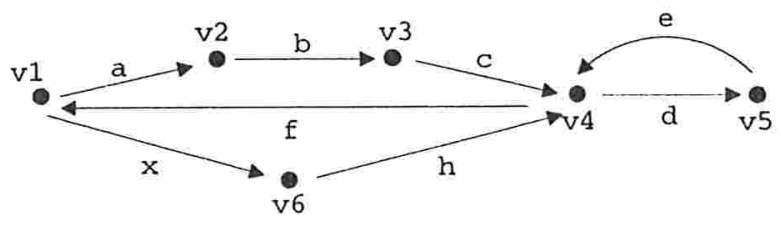

Escolhemos como base multiplicativa o conjunto $\mathcal{P}$ de todos os caminhos em $\Gamma$ e fixamos a ordem de grau e lexicografia em $\mathcal{P}$ com $a<b<\ldots<x<h$. Seja $S=\left\{f_{1}, f_{2}\right\}$, com $f_{1}=a b c-x h$ e $f_{2}=d e$ e considere $v=d e d e+a b c d+f x h+d e$. Computamos então $\operatorname{DIVISÃO}(S, v)$.

Primeiramente, $g \leftarrow v, h \leftarrow 0$ e o algoritmo escolhe $f_{2}$ para a redução feita na linha $5 .^{1}$ Então teremos:

$$
g \leftarrow g-d e f_{2} v_{4}=d e d e+a b c d+f x h+d e-d e d e
$$

Para a segunda redução, o processo escolhe $f_{1}$, obtendo:

$$
g \leftarrow g-v_{1} f_{1} d=a b c d+f x h+d e-v_{1} f_{1} d
$$

Então, $\widehat{g}=x h d$ não é divisível nem por $\widehat{f}_{1}$, nem por $\widehat{f}_{2}$, tornando a condição do laço da linha 4 falsa. Assim, como $g$ é não nulo, teremos:

$$
\begin{aligned}
& h \leftarrow h+x h d=x h d \\
& g \leftarrow g-x h d=f x h+d e
\end{aligned}
$$

Mais uma vez, $\widehat{g}=f x h$ não é divisível pelo termo líder de nenhum elemento de $S$ e teremos:

$$
\begin{aligned}
& h \leftarrow h+f x h=x h d+f x h \\
& g \leftarrow g-f x h=d e
\end{aligned}
$$

Então, o termo líder de $g$ pode ser dividido pelo termo líder de $f_{2}$, donde:

$$
g \leftarrow g-v_{4} f_{2} v_{4}=d e-d e=0
$$

E o algoritmo termina devolvendo $x h d+f x h$.

Uma característica interessante (e um pouco indesejável) do processo descrito acima é que o valor de sua saída pode ser diferente para diferentes escolhas de divisores na linha 4 . O seguinte exemplo mostra essa situação. ${ }^{2}$

\footnotetext{
${ }^{1}$ Note que $f_{2}$ é a única opção neste ponto, mas existe mais de uma forma de dividir $\widehat{g}$ por $\widehat{f_{2}}$. De fato, conforme observado, o processo apresentado nesta seção é não determinístico, de forma que apresentamos a simulação de uma execução possivel deste processo.

${ }^{2} \mathrm{O}$ leitor deve recordar que esta característica do Algoritmo da Divisão também é presente no caso comutativo (vide Exemplo 2.4.1).
} 
Exemplo 4.1.2 Considere $K\langle a, b, c\rangle$. A base multiplicativa escolhida é o conjunto de todas as palavras sobre o alfabeto $X:=\{a, b, c\}$. Fixamos a ordem de grau e lexicografia em $X^{*}$, tomando $c<b<a$.

Seja então $S=\left\{f_{1}, f_{2}, f_{3}\right\}$, com $f_{1}=a b-a c, f_{2}=a a-a c$ e $f_{3}=b a$ e $f=c a a b a$ e computamos DivisÃo $(S, f)$.

Inicialmente, $g \leftarrow f$ e $h \leftarrow 0$.

Com isso, se o algoritmo escolhe $f_{1}$ para a primeira redução da linha 5 , teremos:

$$
g \leftarrow g-c a f_{1} a=c a a c a
$$

Com isso, não resta outra opção, senão escolher $f_{2}$ para a segunda redução (uma vez que nem o termo líder de $f_{1}$ nem o de $f_{3}$ dividem $c a^{2} c a$ ):

$$
g \leftarrow g-c f_{2} c a=c a c c a
$$

Finalmente, cacca não é divisível pelo termo líder de nenhum elemento de $S$ e a saída será cacca.

Por outro lado, se para a primeira redução, o algoritmo tivesse escolhido o elemento $f_{2}$, teríamos ao seguinte:

$$
g \leftarrow g-c f_{2} b a=c a c b a
$$

Assim, resta apenas a opção de escolher $f_{3}$ para a segunda redução e:

$$
g \leftarrow g-\operatorname{cacf}_{3} 1=0
$$

Portanto, a saída para esta seqüência de escolhas será 0 .

Ainda, se o processo escolher $f_{3}$ para a primeira redução, também será devolvido 0 como saída, mas na primeira iteração.

Neste ponto vale comentar que esta dependência da saída do processo Divisão da ordem de escolha de divisores é inerente a propriedades algébricas de $S$ e ao Algoritmo da Divisão e não um produto da vaga descrição de escolha da linha 4 . Com efeito, ainda que consideremos $S$ como um conjunto ordenado, e tenhamos uma forma "canônica" de dividir um monômio por outro ${ }^{3}$, uma alteração na ordem dos elementos do conjunto de entrada $S$ poderá causar saídas diferentes do algoritmo, tal qual o exemplo acima.

Uma conseqüência ainda mais importante dessa dependência de ordem remete ao problema da pertinència. Como deve estar claro para o leitor, um dos grandes interesses no estudo de Bases de Gröbner é a possibilidade de (algoritmicamente) decidir a pertinência de um elemento em um ideal. Assim como fizemos para o caso comutativo na Seção 2.4, temos a intenção de que a saída de uma chamada para o Algoritmo da Divisào, $f^{\prime}=\operatorname{Divisĩo}(S, f)$, decidirá se $f$ pertence ou não ao ideal gerado por $S$. Mas se os valores de $f^{\prime}$ forem diferentes para diferentes ordens dadas nos elementos de $S$, como decidir qual ordem é melhor, ou ainda, qual nos fornece uma resposta correta?

\footnotetext{
${ }^{3}$ No caso das álgebras livres ou de caminhos, poderíamos estabelecer que sempre deve ser feita a divisão "mais à esquerda", por exemplo. Para uma definição mais precisa consulte a Seção 4.1.2.
} 
A resposta para esta pergunta é dada pela Proposição 4.1.4. Assim como ocorre no caso comutativo, se o conjunto $S$ for uma Base de Gröbner, então $f^{\prime}$ independe da ordem de escolha dos elementos de $S$ para efetuar reduções. Isso justifica a maneira vaga com que foi descrita a escolha da linha 4 no algoritmo DIVISÃO.

De fato, quando utilizamos uma Base de Gröbner $G$ em conjunto com o Algoritmo da Divisão, sua saída é sempre a forma normal de $f$ (módulo $\langle G\rangle$ ), independente da ordem em que os elementos de $G$ são considerados para efetuar reduções. Tal fato será demonstrado também na Proposição 4.1.4.

Antes de prosseguirmos, fixamos alguma notação e terminologia.

Definição 4.1.1 (Redução sobre um conjunto) Tome $S$ um subconjunto finito de $R$ e $f$ um elemento de $R$. Diremos que $f$ se reduz para $f^{\prime}$ sobre $S$ (denotando esse fato por $f \Rightarrow_{S} f^{\prime}$ ) quando a saída do Algoritmo da Divisão ao receber $S$ e $f$ como parâmetros for $f^{\prime}$ para alguma seqüência de escolhas de divisores nas execuções da linha 4 do algoritmo DIVISÃO.

Ainda, se existem $b, b^{\prime}$ em $\mathcal{B}$ e $v$ em $R$, tais que $b \widehat{v} b^{\prime}=\widehat{f}$, diremos que o elemento $f-\frac{c l(f)}{c l(v)} b v b^{\prime}$ é uma redução simples de $f$ por $v$ e ao construir uma redução simples de $f$ por $v$, diremos que estamos reduzindo $f$ por $v$.

Uma observação importante é que o termo líder de uma redução simples de $f$ é sempre estritamente menor que o termo líder de $f$. Resumimos esse fato na seguinte afirmação para conveniência do leitor.

Afirmação 4.1.1 Sejam $f, v$ e $f^{\prime}$ em $R$, de forma que $f^{\prime}$ é uma redução simples de $f$ por $v$. Então $\hat{f}^{\prime}<\widehat{f}$.

Em seguida apresentamos algumas propriedades importantes do Algoritmo da Divisão em uma série de lemas e proposições. Iniciamos com sua correção e término.

Proposição 4.1.2 Seja $R$ uma álgebra munida de uma base multiplicativa $\mathcal{B}$ sobre a qual está fixada uma ordem admissivel. Então, se $S=\left\{f_{1}, f_{2}, \ldots, f_{s}\right\}$ é um subconjunto finito de $R$ e $f$ é um elemento de $R$, DIVISÃo $(S, f)$ termina em um número finito de passos e sua saída (denotada por $\left.f^{\prime}\right)$ é tal que:

1. Para cada $b$ no suporte de $f^{\prime}, b \leq \widehat{f}$ e b não é divisível pelo termo líder de nenhum $f_{i}$, para $1 \leq i \leq s$.

2. Existe um inteiro $k$, elementos $b_{1}, b_{1}^{\prime}, b_{2}, b_{2}^{\prime}, \ldots, b_{k}, b_{k}^{\prime}$ de $\mathcal{B}$ e coeficientes $\alpha_{1}, \alpha_{2}, \ldots, \alpha_{k}$ tais que

$$
f=\sum_{j=1}^{k} \alpha_{j} b_{j} f_{i,} b_{j}^{\prime}+f^{\prime}
$$

satisfazendo $b_{j} \widehat{f_{i}} b_{j}^{\prime} \leq \widehat{f}$, para $1 \leq j \leq k$.

3. Uma e somente uma das afirmações abaito é verdadeira: 
(a) $\widehat{f}=\widehat{f^{\prime}}$ e $b_{j} \widehat{f_{i_{j}}} b_{j}^{\prime} \neq \widehat{f}$, para $1 \leq j \leq k$.

(b) $\widehat{f}=b_{h} \widehat{f_{i_{h}}} b_{h}^{\prime}$, para algum $1 \leq h \leq k, \widehat{f} \neq \widehat{f}^{\prime}$ e $b_{j} \widehat{f_{i_{j}}} b_{j}^{\prime} \neq \widehat{f}$, para todo $j \neq h$.

Demonstração. Para verificar que o processo termina em um número finito de passos, basta utilizar o fato de termos uma ordem admissível em $\mathcal{B}$ (que, por definição, é uma boa ordem), a Proposição 1.1.1 e observar que o termo líder de $g$ diminui a cada iteração do laço das linhas 3 a 8 e também a cada iteração do laço das linhas 4 e 5 . Com isso, se algum desses laços nunca termina, o conjunto dos termos líderes dos valores de $g$ a cada iteração formam uma cadeia descendente infinita, o que nos leva a uma contradição. Portanto, o procedimento termina em um número finito de passos.

Em seguida, note que, como comentado acima, o termo líder de $g$ diminui a cada iteração, de forma que a seguinte desigualdade é válida em qualquer ponto da execução do algoritmo: $\widehat{g} \leq \widehat{f}$.

Para verificar a primeira propriedade do enunciado da proposição, precisamos analisar o valor de $h$ ao término do algoritmo. Note que a única operação que adiciona elementos ao suporte de $h$ é a atribuição da linha 7 e que essa atribuição adiciona justamente $\widehat{g}$ a seu suporte. Mas essa atribuição só será executada quando $g$ for diferente de 0 e quando a condição da instrução enquanto da linha 4 for falsa, ou seja, quando $\widehat{g}$ não for divisível pelo termo líder de nenhum elemento de $S$. Com isso, cada elemento $b$ adicionado ao suporte de $h$ é tal que não existe $f_{i}$ em $S(1 \leq i \leq s)$ de forma que $\widehat{f}_{i}$ divida $b$. Ademais, pela observação anterior, $\widehat{g} \leq \widehat{f}$ a cada iteração, o que encerra a demonstração da propriedade 1.

Para a segunda propriedade, mostraremos que o algoritmo possui o seguinte invariante:

Invariante I1 No início de cada execução da linha 3 do algoritmo, $f=g+$ $h+\sum_{j=1}^{k} \alpha_{j} b_{j} f_{i_{j}} b_{j}^{\prime}$, para algum inteiro $k$, alguns $f_{i_{j}}$ em $S, b_{j}, b_{j}^{\prime}$ em $\mathcal{B}$ e coeficientes $\alpha_{1}, \alpha_{2}, \ldots, \alpha_{k}$, tais que $b_{j} \widehat{f_{i_{j}}} b_{j}^{\prime} \leq \widehat{f}$, para $1 \leq j \leq k$.

No início da primeira execução da linha 3 , temos que $h=0$ e $f=g$, logo o invariante é válido neste momento. Mostraremos que se o invariante é válido no início da $i$-ésima execução da linha 3 (e que não se trata da última execução dessa linha), então ele será válido no início da $(i+1)$-ésima execução dessa mesma linha.

Considere agora a $i$-ésima execução da linha 3 . O laço das linhas 4 e 5 encontram elementos $f_{i_{j}}, b_{j}, b_{j}^{\prime}$ da forma descrita no invariante, com a propriedade de que $b_{j} \widehat{f_{i_{j}}} b_{j}^{\prime}=$ $\widehat{g} \leq \widehat{f}$ e subtrai elementos da forma $\alpha_{j} b_{j} f_{i_{j}} b_{j}^{\prime}$ de $g$, de forma que o invariante continua válido após o término desse laço. Ĺma vez terminado o laço, nada é feito se $g$ for nulo e o invariante continua sendo válido no início da $(i+1)$-ésima execução da linha 3 neste caso.

Caso $g$ seja não nulo, o algoritmo faz $g-g-\operatorname{cl}(g) \widehat{g}$ e $h \leftarrow h+\operatorname{cl}(g) \widehat{g}$, de forma que a soma $g+h$ se mantém inalterada e o invariante continua sendo válido após a execução da condicional da linha 6 . Com isso, o invariante é válido no início da $(i+1)$-ésima execução da linha 3. 
Portanto, acabamos de mostrar que o Invariante $I 1$ é válido no início de cada execução da linha 3 do Algoritmo da Divisão.

Em particular, o Invariante $I 1$ é válido no início da última execução da linha 3. Mas, como se trata da última execução dessa linha, temos que sua condição de teste $(g \neq 0)$ é necessariamente falsa (caso contrário, esta linha seria executada pelo menos mais uma vez).

Com isso, imediatamente antes da execução da linha 9 do algoritmo, teremos que $g=0 \mathrm{e}$ $f=h+\sum_{j=1}^{k} \alpha_{j} b_{j} f_{i_{j}} b_{j}^{\prime}$, para algum inteiro $k$, alguns $f_{i_{j}}$ em $S, b_{j}, b_{j}^{\prime}$ em $\mathcal{B}$ e coeficientes $\alpha_{1}, \alpha_{2}, \ldots, \alpha_{k}$, satisfazendo $b_{j} \widehat{f_{i_{j}}} b_{j}^{\prime} \leq \widehat{f}$. Mas imediatamente antes da execução da linha 9 do algoritmo, $h$ é igual à saída do algoritmo $\left(f^{\prime}\right)$ e segue o resultado.

Finalmente, a terceira propriedade pode ser vista sem grande dificuldade pelo funcionamento do algoritmo e pelo fato de que o termo líder de $g$ diminui a cada iteração.

O exemplo abaixo mostra que nem todo elemento que se escreve na forma da condição 2 da Proposição 4.1.2 corresponde a uma saída do Algoritmo da Divisão.

Exemplo 4.1.3 Tome $R=K\langle a, b, c, d\rangle$ e fixe a ordem de grau e lexicografia em $\{a, b, c, d\}^{*} \operatorname{com} a>b>c>d$. Seja então $S:=\left\{f_{1}, f_{2}\right\}$, onde $f_{1}=a^{3}-a b$ e $f_{2}=a b-c^{2}$. Considere

$$
f=f_{1} b-a^{2} f_{2}+f_{1} d^{2}=a^{3} d^{2}+a^{2} c^{2}-a b d^{2}-a b^{2}
$$

Note que $f$ está escrito como nas condições do enunciado da Proposição 4.1 .2 (com $\left.f^{\prime}=0\right)$. Mostraremos que não existe nenhuma seqüência de escolhas de divisores para o Algoritmo da Divisão que produza zero como saída. Computamos então DIVISÃo $(S, f)$.

Na primeira iteração, o algoritmo não tem outra opção senão escolher $f_{1}$ para efetuar a redução. Note ainda que que existe uma única forma de dividir $\widehat{g}=a^{3} d^{2}$ por $\widehat{f}_{1}$. Então teremos:

$$
g \leftarrow g-1 f_{1} d^{2}=a^{2} c^{2}-a b^{2}
$$

Em seguida, vemos que $\widehat{g}=a^{2} c^{2}$ não é divisível nem por $\widehat{f}_{1}$, nem por $\widehat{f}_{2}$ e teremos:

$$
\begin{aligned}
& h \leftarrow h+a^{2} c^{2}=a^{2} c^{2} \\
& g \leftarrow g-a^{2} c^{2}=-a b^{2}
\end{aligned}
$$

Com isso, resta apenas a opção de utilizar $f_{2}$ para efetuar a próxima redução. Mais uma vez, há apenas uma maneira de dividir $\widehat{g}=a b^{2}$ por $\widehat{f}_{2}$. Então:

$$
g \leftarrow g+1 f_{2} b=-c^{2} b
$$

Novamente, $\widehat{g}=c^{2} b$ não é divisível pelo termo líder de nenhum elemento de $S$, levando o processo a realizar as seguintes atribuições: 


$$
\begin{aligned}
& h \leftarrow h-c^{2} b=a^{2} c^{2}-c^{2} b \\
& g \leftarrow g+c^{2} b=0
\end{aligned}
$$

E a saída do Algoritmo da Divisão será $a^{2} c^{2}-c^{2} b \neq 0$. Ainda, como a cada iteração há apenas uma escolha possível de divisores para $\widehat{g}$, segue que $f$ não se reduz para zero sobre $S$.

O corolário a seguir é conseqüência imediata da Proposição 4.1.2.

Corolário 4.1.3 Seja $S$ um subconjunto finito de $R$ e $f$ um elemento de $R$. Se $f^{\prime}=\operatorname{DiviSÃO}(S, f)$, então

$$
\langle S \cup\{f\}\rangle=\left\langle S \cup\left\{f^{\prime}\right\}\right\rangle
$$

O seguinte resultado mostra a importância de se utilizar Bases de Gröbner em conjunto com o Algoritmo da Divisão.

Proposição 4.1.4 Seja $R$ uma álgebra com base multiplicativa $\mathcal{B}$ sobre a qual está fixada uma ordem admissivel. Sejam ainda $G \subset R$ uma Base de Gröbner finita, $f$ um elemento de $R$ e denote por $I$ o ideal gerado por $G$. Então a saída do Algoritmo da Divisão (denotada por $f^{\prime}$ ) ao receber $G$ e $f$ como entrada é $N_{I}(f)$. Em particular, $f^{\prime}$ é independente da escolha de divisores na linha 4 do algoritmo.

Demonstração. Suponha que $f$ se reduz para $f^{\prime}$ sobre $G$. Então, pela Proposição 4.1.2, segue que

$$
f=\sum_{j=1}^{k} \alpha_{j} b_{j} f_{i_{j}} b_{j}^{\prime}+f^{\prime}
$$

com a propriedade de que nenhum elemento de $\operatorname{supp}\left(f^{\prime}\right)$ é divisível pelo termo líder de nenhum elemento de $G$.

Mas, dado que $G$ é uma Base de Gröbner, segue que $f^{\prime}$ pertence a span(Norm(I)). Claro que $\sum_{j=1}^{k} \alpha_{j} b_{j} f_{i j} b_{j}^{\prime}$ pertence a $I$. Logo, pelo Teorema 3.1.6, temos que $f^{\prime}=N_{I}(f)$. A unicidade da saída do algoritmo segue então da unicidade de $N_{I}(f)$ (dada pelo Teorema 3.1.6) e do fato de termos tomado uma seqüência qualquer de escolhas de divisores na linha 4 do algoritmo.

De maneira análoga ao que foi feito no Capítulo 2, essa proposição nos mostra que o Algoritmo da Divisão pode ser utilizado em conjunto com Bases de Gröbner para resolver o problema da pertinência para ideais (e, conseqüentemente o da igualdade). Ademais, ela nos mostra a importância de utilizarmos Bases de Gröbner em conjunto com o Algoritmo da Divisão e que, neste caso, pode-se escolher qualquer forma para implementar as escolhas da linha 4 do algoritmo (ao menos do ponto de vista de correção do algoritmo).

Uma observação interessante é que, dado um elemento $f$ de $R$, não é necessária uma Base de Gröbner $G$ completa para que o Algoritmo da Divisão encontre $N(f)$. Com efeito, os elementos de 
$G$ escolhidos na linha 4 do algoritmo devem dividir elementos do suporte de $f$. Logo, a presença de uma ordem admissível nos garante que apenas elementos de $G$ com termos líderes menores ou iguais a $\widehat{f}$ podem ser escolhidos pelo Algoritmo da Divisão.

Com o intuito de formalizar esses comentários, definimos a noção de Base de Gröbner truncada.

Definição 4.1.2 (Base de Gröbner Truncada) Dada uma Base de Gröbner G e um monômio $b$, definimos a Base de Gröbner truncada em b obtida a partir de $G$ como

$$
G_{b}=\{g \in G \mid \widehat{g} \leq b\}
$$

Assim obtemos o seguinte corolário como conseqüência dos comentários acima e da Proposição 4.1.4.

Corolário 4.1.5 Seja $f$ um elemento de $R, G \subset R$ uma Base de Gröbner e denote por I o ideal gerado por $G$. Nestas condições, a saída do Algoritmo da Divisão (denotada por $f^{\prime}$ ) ao receber $G_{\hat{f}}$ e $f$ como entrada é $N_{I}(f)$. Em particular, $f^{\prime}$ é independente da escolha de divisores na linha 4 do algoritmo.

Um comentário interessante a respeito de Bases de Gröbner truncadas é que existem ordens admissíveis que possuem a seguinte propriedade: dado um elemento $b$ de $\mathcal{B}$, existe apenas um número finito de elementos menores ou iguais a $b$. Ordens que satisfazem essa propriedade são chamadas em alguns trabalhos de seqüenciais [Mor94].

A ordem de grau e lexicografia definida no Capítulo 3 é um exemplo de ordem admissível seqüencial (a verificação desse fato é bastante natural e deixada a cargo do leitor). Por outro lado, existem ordens admissíveis que não são seqüenciais: podemos citar a ordem definida no Exemplo 3.2.1 (de acordo com essa ordem, qualquer potência de $b$ é menor que $a$ ).

Com isso, se a ordem admissível fixada em $\mathcal{B}$ for seqüencial, dado um elemento $f$ de $R$, sempre existe uma Base de Gröbner truncada em $\widehat{f}$ que seja finita. Contudo, a Seção 4.4 nos mostrará que não é possível encontrar um algoritmo que construa tal conjunto, apesar de sua finitude.

Seguimos com mais algumas caracterizações da saída do Algoritmo da Divisão que nos serão úteis adiante. Para os resultados que seguem, denote por $R$ uma álgebra com uma base multiplicativa $\mathcal{B}$ sobre a qual está fixada uma ordem admissível. Seja ainda $S=\left\{f_{1}, f_{2}, \ldots, f_{s}\right\}$ um subconjunto finito de $R, f$ um elemento de $R$ e denote por $f^{\prime}$ a saída do Algoritmo da Divisão ao receber como entrada $S$ e $f$, ou seja $f^{\prime}=\operatorname{Divis} \tilde{O}(S, f)$.

Lema 4.1.6 A cada execução da linha 7 do Algoritmo da Divisão, $\widehat{g}$ não pertence a $\operatorname{supp}(h)$.

Demonstração. A verificação do lema segue imediatamente da observação de que o termo líder de $g$ diminui a cada execução do laço das linhas 3 a 8 .

Lma consequiència importante do lema acima é que ele nos mostra que um monômio adicionado ao suporte de $h$ nunca é cancelado.

Lema 4.1.7 O monômio $\widehat{f}$ pertence ao suporte de $f^{\prime}$ se e somente se $\widehat{f}$ não for divisivel pelo termo líder de nenhum elemento de $S$. 
Demonstração. A propriedade 1 descrita na Proposição 4.1 .2 nos mostra claramente que se $\widehat{f}$ pertence ao suporte de $f^{\prime}$, então ele não será divisível por nenhum $\widehat{f}_{i}$, para $1 \leq i \leq s$. Vejamos então a implicação reversa.

Suponha então que $\widehat{f}$ não é divisível pelo termo líder de nenhum $f_{i}$ (para $1 \leq i \leq s$ ) e vejamos que ele pertence a $\operatorname{supp}\left(f^{\prime}\right)$. Mas então, na primeira iteração do algoritmo, a condição da linha 4 será falsa com $g=f \neq 0$ e as linhas 7 e 8 serão executadas, fazendo com que $\widehat{f}$ (que é igual a $\widehat{g}$ nessa iteração) seja adicionado a $\operatorname{supp}(h)$. Claramente, esse valor não poderá ser cancelado no suporte de $h$ pelo Lema 4.1.6.

O seguinte corolário é conseqüência imediata do lema que acabamos de demonstrar.

Corolário 4.1.8 Se um elemento $f$ de $R$ se reduz para zero sobre $S$, então $\widehat{f}$ é divisível por $\widehat{f}_{i}$, para algum $f_{i}$ em $S$.

O resultado apresentado a seguir é conseqüência imediata desse último corolário.

Corolário 4.1.9 Denote por $I=\langle S\rangle$ e suponha que todo elemento de $I$ se reduz para zero sobre $S$. Então $S$ é uma Base de Gröbner.

A observação a seguir segue imediatamente da definição de redução sobre um conjunto e da descrição do Algoritmo da Divisão. Ela será útil ao provarmos a correção do Procedimento de Mora.

Afirmação 4.1.10 O elemento $f$ se reduz para zero sobre $S \cup\left\{f^{\prime}\right\}$.

Denotaremos por $\operatorname{red}_{S}$ a relação em $R$ que associa cada elemento $f$ de $R$ ao conjunto

$$
\operatorname{red}_{S}(f):=\{g \in R \mid f \text { se reduz para } g \text { sobre } S\}
$$

Quando o contexto tornar claro qual o conjunto $S$ a que nos referimos, denotaremos essa relação simplesmente por red.

A seguinte afirmação segue imediatamente do funcionamento do Algoritmo da Divisão e do Lema 4.1.6.

Afirmação 4.1.11 Consideremos o início de uma i-ésima execução da linha 4 do Algoritmo da Divisão ao receber $f \in R$ e $S \subseteq R$ como parâmetros de entrada. Denotamos por a e b os valores das variáveis $h$ e $g$ neste ponto, respectivamente.

Se b se reduz para d sobre $S$, então $f$ se reduz para $d+a$ sobre $S$. Em outras palavras, $\operatorname{red}(b)+$ $\{a\} \subseteq \operatorname{red}(f)$.

E particular, se $f$ é uma redução simples de $f^{\prime}$, então $\operatorname{red}\left(f^{\prime}\right) \subseteq \operatorname{red}(f)$.

A fim de tornar a notação utilizada na proposição a seguir mais leve, denotaremos um conjunto unitário $\{a\}$ por a (quando não houver possibilidade de confusão).

Proposição 4.1.12 Sejam $f, f_{1}$ e $f_{2}$ elementos de $R, S=\left\{s_{1}, s_{2}, \ldots, s_{n}\right\}$ um subconjunto de $R$ e $\lambda$ um escalar näo nulo. Então: 
1. Se $f=\lambda f_{1}$, então $\operatorname{red}(f)=\lambda \operatorname{red}\left(f_{1}\right)$.

2. Se $f=f_{1}+f_{2}$, então $\operatorname{red}(f) \subseteq\left(\operatorname{red}\left(f_{1}\right)+\operatorname{red}\left(f_{2}\right)\right)$.

Demonstração. A proposição é trivialmente verdadeira se algum elemento dentre $f, f_{1}$ e $f_{2}$ for zero (note que, se $f=0$, então $f_{1}=-f_{2}$ e o segundo caso segue do primeiro, nesta situação). Dessa forma, assumimos no que segue que os três são não nulos.

Vejamos a primeira parte. Como $f_{1}=(1 / \lambda) f$, fica evidente que é suficiente mostrar que $\operatorname{red}(f) \subseteq \lambda \operatorname{red}\left(f_{1}\right)$, para todos $f, f_{1}$ e $\lambda$. Mostraremos essa inclusão por redução ao absurdo.

Para tanto, suponha que o lema é falso e tomemos um conjunto $S$ e um contra exemplo $f$ de termo líder mínimo. Então $f=\lambda f_{1}$, para algum $f_{1}$ em $R$ e existe $d$ em $\operatorname{red}(f)$ de modo que $d$ não pertence a $\lambda \operatorname{red}\left(f_{1}\right)$.

Claro que o Algoritmo de Divisão faz uma sequiência de escolhas de divisores para reduzir $f$ para $d$. Seja $\left(b_{1} s_{i_{1}} b_{1}^{\prime}, b_{2} s_{i_{2}} b_{2}^{\prime}, \ldots, b_{m} s_{i_{m}} b_{m}^{\prime}\right.$ ) essa seqüência (cada $b_{j}$ e $b_{j}^{\prime}$ pertencem a $\mathcal{B}$ e cada $s_{i_{j}}$ pertence a $S$ ).

Mostraremos que $d \in \lambda \operatorname{red}\left(f_{1}\right)$. Para tanto, consideramos uma chamada para o Algoritmo da Divisão, $\operatorname{DivisÃo}(S, f)$. Então, na primeira iteração do laço das linhas 4 e 5 , existem duas possibilidades: $\widehat{f}=b_{1} s_{i_{1}} b_{1}^{\prime}$ ou o termo líder de $f$ não é divisível pelo termo líder de nenhum elemento de $S$. Examinamos essas duas possibilidades em separado a seguir:

- O termo líder de $f$ é igual a $b_{1} s_{i_{1}} b_{1}^{\prime}$.

Neste caso, uma vez que $f=\lambda f_{1}$, é imediato ver que $\widehat{f}_{1}=\widehat{f}$ e $\operatorname{cl}(f)=\lambda c l\left(f_{1}\right){ }^{4}$ Assim, uma escolha possível para o Algoritmo da Divisão na primeira iteração ao computar DiVisÃo $\left(S, f_{1}\right)$ é $b_{1} s_{i_{1}} b_{1}^{\prime}$. Assim, definimos:

$$
f_{1}^{\prime}:=f_{1}-\alpha b_{1} s_{i_{1}} b_{1}^{\prime} \text { e } f^{\prime}:=f-\lambda \alpha b_{1} s_{i_{1}} b_{1}^{\prime}
$$

(com $\left.\alpha=\operatorname{cl}\left(f_{1}\right) / \operatorname{cl}\left(s_{i_{1}}\right)\right)$. Note que $f^{\prime}$ e $f_{1}^{\prime}$ são reduções simples para $f$ e $f_{1}$, respectivamente. Assim, $f^{\prime}=\lambda f_{1}^{\prime}$. Logo, se $f^{\prime}$ ou $f_{1}^{\prime}$ for zero, $d=0$ e não há mais o que mostrar. Assumimos então que ambos $f^{\prime}$ e $f_{1}^{\prime}$ são não nulos. Nesse caso, observamos também que $\widehat{f}^{\prime}<\widehat{f}$ e $\widehat{f}_{1}^{\prime}<\widehat{f}_{1}$.

Mas então, pela minimalidade de $\widehat{f}$, segue que $\operatorname{red}\left(f^{\prime}\right) \subseteq \lambda \operatorname{red}\left(f_{1}^{\prime}\right)$.

Não é difícil ver que $d$ pertence a red $\left(f^{\prime}\right)$ pelo funcionamento do Algoritmo da Divisão. Para ver esse fato, basta tomar a seguinte seqüência de escolhas de divisores: $\left(b_{2} s_{i_{2}} b_{2}^{\prime} \ldots, b_{m} s_{i_{m}} b_{m}^{\prime}\right)$ e notar que $h=0$ após reduzir $f$ para $f^{\prime}$.

Mas então, a Afirmação 4.1.11 nos mostra que $\operatorname{red}\left(f_{1}^{\prime}\right) \subset \operatorname{red}\left(f_{1}\right)$, donde $\lambda \operatorname{red}\left(f_{1}^{\prime}\right) \subseteq$ $\lambda \operatorname{red}\left(f_{1}\right)$. Assim,

$$
d \in \operatorname{red}\left(f^{\prime}\right) \subseteq \lambda \operatorname{red}\left(f_{1}^{\prime}\right) \subseteq \lambda \operatorname{red}\left(f_{1}\right)
$$

nos levando a uma contradição e concluindo a demonstração neste caso.

\footnotetext{
${ }^{4}$ Lembramos que, por ser um corpo, $K$ é um domínio de integridade. Portanto, $\operatorname{supp}(f)=\operatorname{supp}\left(f_{1}\right)$.
} 
- O termo líder de $f$ não é divisível pelo termo líder de nenhum elemento de $S$.

Pelo mesmo argumento dado acima, $\widehat{f}_{1}$ também não é divisível pelo termo líder de nenhum elemento de $S$. Então consideramos a primeira iteração de uma chamada para $\operatorname{DIVISÃo}\left(S, f_{1}\right)$ e definimos:

$$
f_{1}^{\prime}:=f_{1}-\operatorname{cl}\left(f_{1}\right) \widehat{f} \text { e } f^{\prime}:=f-\lambda c l\left(f_{1}\right) \widehat{f}
$$

Novamente, $\widehat{f}_{1}^{\prime}<\widehat{f}_{1}, \widehat{f}^{\prime}<\widehat{f}$ e $f^{\prime}=\lambda f_{1}^{\prime}{ }^{5}$ Logo, pela minimalidade de $\widehat{f}$, temos que $\operatorname{red}\left(f^{\prime}\right) \subseteq \lambda \operatorname{red}\left(f_{1}^{\prime}\right)$. Ainda a Afirmação 4.1.11 nos diz que $\operatorname{red}\left(f_{1}^{\prime}\right)+c l\left(f_{1}\right) \widehat{f} \subseteq$ $\operatorname{red}\left(f_{1}\right)$.

Pelo funcionamento do Algoritmo da Divisão e pelo Lema 4.1.6, não é difícil ver que $d-c l(f) \widehat{f}$ pertence a $\operatorname{red}\left(f^{\prime}\right) \subseteq \lambda \operatorname{red}\left(f_{1}^{\prime}\right)$. Logo,

$$
\frac{d-c l(f) \hat{f}}{\lambda}+\operatorname{cl}\left(f_{1}\right) \widehat{f} \subseteq \operatorname{red}\left(f_{1}\right)
$$

e concluímos que

$$
d \in \lambda \operatorname{red}\left(f_{1}\right)
$$

como queríamos.

Isso conclui a demonstração da primeira parte da proposição. Nos voltamos em seguida para demonstrar a segunda.

A prova da segunda parte também segue por contradição. Tome $S$ um subconjunto qualquer de $R$ para o qual a segunda parte do lema é falsa e seja $f$ um contra exemplo de termo líder mínimo. Então existem $d, f_{1}$ e $f_{2}$ em $R$, de modo que $f=f_{1}+f_{2}, f$ se reduz para $d$ sobre $S$, mas $d$ não pertence a $\operatorname{red}\left(f_{1}\right)+\operatorname{red}\left(f_{2}\right)$.

Por um lado, tomemos $M_{k}:=\left\{b \in \operatorname{supp}\left(f_{k}\right) \mid b>\widehat{f}\right\}$, para $1 \leq k \leq 2$. É importante notar que todo monômio em $M_{1}$ ou em $M_{2}$ deve ser cancelado na soma $f_{1}+f_{2}$. Logo, $M_{1}=M_{2}$ e podemos denotar $M=M_{1}=M_{2}$. Ainda, se $b$ pertence a $M$, os coeficientes de $b$ em $f_{1}$ e $f_{2}$ são opostos.

Considere uma chamada para $\operatorname{DIVISÃo}\left(S, f_{1}\right)$. Podemos considerar a execução do algoritmo até a primeira iteração em que a variável $g$ contém um elemento com termo líder menor ou igual ao termo líder de $f$ (ou é zero). Denotemos o valor de $g$ nesse ponto por $f_{1}^{\prime}$ e o da variável $h$ por $t_{1}$. Podemos considerar ainda a seqüência de escolhas de divisores utilizados até esse momento: $\left(a_{1} s_{j_{1}} a_{1}^{\prime}, a_{2} s_{j_{2}} a_{2}^{\prime}, \ldots, a_{v} s_{j_{v}} a_{v}^{\prime}\right)$. Note ainda que $\operatorname{supp}\left(t_{1}\right) \subseteq M, a_{x} \widehat{j_{x}} a_{x}^{\prime} \in M$, para todo $1 \leq x \leq v$ e ou $\widehat{f_{1}^{\prime}} \leq \widehat{f}$ ou $f_{1}^{\prime}=0$. Assim, teremos: ${ }^{6}$

$$
f_{1}=t_{1}+f_{1}^{\prime}+\sum_{x=1}^{v} \beta_{x} a_{x} s_{j_{x}} a_{x}^{\prime}
$$

\footnotetext{
${ }^{5}$ Aqui, como no caso anterior, nada mais resta a demonstrar se $f_{1}^{\prime}$ ou $f^{\prime}$ for nulo.

${ }^{6} \mathrm{Na}$ realidade, esta igualdade é dada pelo Invariante $I 1$ da demonstração da Proposição 4.1.2.
} 
Analogamente, podemos considerar Divisão $\left(S, f_{2}\right)$. Mas como, todo elemento de $M$ pertence ao suporte de $f_{2}$, podemos tomar a mesma seqüência de escolhas de divisores. Note que os coeficientes utilizados para efetuar as reduções serão opostos, que $\widehat{f}_{2}^{\prime} \leq \widehat{f}$ se $f_{2}^{\prime}$ for não nulo e que $t_{2}=-t_{1}$. Portanto, teremos:

$$
f_{2}=t_{2}+f_{2}^{\prime}-\left(\sum_{x=1}^{v} \beta_{x} a_{x} s_{j_{x}} a_{x}^{\prime}\right)
$$

Denote ainda por $\alpha_{1}$ o coeficiente de $\widehat{f}$ em $f_{1}^{\prime}$ (possivelmente zero) e por $\alpha_{2}$ o coeficiente de $\widehat{f}$ em $f_{2}^{\prime}$ (possivelmente zero).

Por outro lado, é claro pelo funcionamento do Algoritmo da Divisão que, para reduzir $f$ para $d$, o algoritmo faz uma seqüência de escolhas de elementos da forma $b s_{i} b^{\prime}$ (com $b$ e $b^{\prime}$ em $\mathcal{B}$ e $s$ em $S$ ) para efetuar as reduções da linha 5 . Seja $\left(b_{1} s_{i_{1}} b_{1}^{\prime}, b_{2} s_{i_{2}} b_{2}^{\prime}, \ldots, b_{m} s_{i_{m}} b_{m}^{\prime}\right)$ essa seqüência.

Como na primeira parte, temos duas possibilidades: $b_{1} s_{i_{1}} b_{1}^{\prime}=\widehat{f}$ ou $\widehat{f}$ não é divisível pelo termo líder de nenhum elemento de $S$. Analisamos cada caso em separado:

- O termo líder de $f$ é igual a $b_{1} s_{i_{1}} b_{1}^{\prime}$.

Denotamos então por $t=f-\alpha b_{1} s_{i_{1}} b_{1}^{\prime}$ (onde $\alpha=\operatorname{cl}(f) / c l\left(s_{i_{1}}\right)$ ). Claro que $\widehat{t}<\widehat{f}$ (se $t$ for não nulo) e $t$ se reduz para $d$ sobre $S$ (basta tomar a escolha de divisores $\left.\left(b_{2} s_{i_{2}} b_{2}^{\prime}, \ldots, b_{m} s_{i_{m}} b_{m}^{\prime}\right)\right)$.

Como $f=f_{1}+f_{2}=f_{1}^{\prime}+f_{2}^{\prime}$, temos que $\alpha=\alpha_{1}+\alpha_{2}$. Assim,

$$
f_{1}^{\prime}-\alpha_{1} b_{1} s_{i_{1}} b_{1}^{\prime}+f_{2}^{\prime}-\alpha_{2} b_{1} s_{i_{1}} b_{1}^{\prime}=f-\alpha b_{1} s_{i_{1}} b_{1}^{\prime}=t
$$

Se denotamos $f_{1}^{\prime \prime}=f_{1}^{\prime}-\alpha_{1} b_{1} s_{i_{1}} b_{1}^{\prime}$ e $f_{2}^{\prime \prime}=f_{2}^{\prime}-\alpha_{2} b_{1} s_{i_{1}} b_{1}^{\prime}$, vemos que $t=f_{1}^{\prime \prime}+f_{2}^{\prime \prime}$. Ademais, como assumimos que o termo líder de $f_{1}^{\prime}$ (resp. $f_{2}^{\prime}$ ) é menor ou igual ao termo líder de $f_{1}$ (resp. $f_{2}$ ) (se for não nulo), temos que $f_{1}^{\prime \prime}$ (resp. $f_{2}^{\prime \prime}$ ) é igual a $f_{1}^{\prime}$ (resp. $f_{2}^{\prime}$ ), se $\alpha_{1}$ (resp. $\alpha_{2}$ ) for zero ou $f_{1}^{\prime \prime}$ (resp. $f_{2}^{\prime \prime}$ ) é uma redução simples de $f_{1}^{\prime}$ (resp. $\left.f_{2}^{\prime}\right)$.

Assim, podemos utilizar a Afirmação 4.1 .11 e as Equações 4.1 e 4.2 para concluir:

$$
\operatorname{red}\left(f_{1}^{\prime \prime}\right) \subseteq \operatorname{red}\left(f_{1}^{\prime}\right) \text { e } \operatorname{red}\left(f_{1}^{\prime}\right)+t_{1} \subseteq \operatorname{red}\left(f_{1}\right)
$$

$\mathrm{e}$

$$
\operatorname{red}\left(f_{2}^{\prime \prime}\right) \subseteq \operatorname{red}\left(f_{2}^{\prime}\right) \operatorname{ered}\left(f_{2}^{\prime}\right)+t_{2} \subseteq \operatorname{red}\left(f_{2}\right)
$$

Mas como $t_{1}=-t_{2}$, temos que

$$
\operatorname{red}\left(f_{1}^{\prime \prime}\right)+\operatorname{red}\left(f_{2}^{\prime \prime}\right) \subseteq \operatorname{red}\left(f_{1}^{\prime}\right)+\operatorname{red}\left(f_{2}^{\prime}\right) \subseteq \operatorname{red}\left(f_{1}\right)+\operatorname{red}\left(f_{2}\right)
$$

Portanto, se $t=0$, $d$ será zero e temos que $f_{1}^{\prime \prime}+f_{2}^{\prime \prime}=0$, donde $f_{1}^{\prime \prime}=-f_{2}^{\prime \prime}$. Logo, pela primeira parte do lema $\operatorname{red}\left(f_{1}^{\prime \prime}\right)=-1 \operatorname{red}\left(f_{2}^{\prime \prime}\right)$ e concluímos que $0 \in$ $\operatorname{red}\left(f_{1}^{\prime \prime}\right)+\operatorname{red}\left(f_{2}^{\prime \prime}\right)$. O resultado nesse caso segue então das inclusões acima. 
Por outro lado, se $t \neq 0$, como $\widehat{t}<\widehat{f}$, segue da minimalidade de $\widehat{f}$, que $d$ pertence a $\operatorname{red}\left(f_{1}^{\prime \prime}\right)+\operatorname{red}\left(f_{2}^{\prime \prime}\right)$. Logo, $d$ pertence a $\operatorname{red}\left(f_{1}\right)+\operatorname{red}\left(f_{2}\right)$ pelas inclusões destacadas acima.

- O termo líder de $f$ não é divisível pelo termo líder de nenhum elemento de $S$.

Neste caso, a prova segue de forma análoga ao argumento anterior, tomando $t=$ $f-c l(f) \widehat{f}, f_{1}^{\prime \prime}=f_{1}^{\prime}-\alpha_{1} \widehat{f}$ e $f_{2}^{\prime \prime}=f_{2}^{\prime}-\alpha_{2} \widehat{f}$, com $\alpha_{1}$ (resp. $\left.\alpha_{2}\right)$ o coeficiente de $\widehat{f}$ em $f_{1}^{\prime}$ (resp. $f_{2}^{\prime}$ ).

É preciso apenas atentar para as seguintes diferenças com relação ao caso acima:

$-d-c l(f) \widehat{f} \in \operatorname{red}(t)$.

$-\operatorname{red}(t) \subseteq \operatorname{red}\left(f_{1}^{\prime \prime}\right)+\operatorname{red}\left(f_{2}^{\prime \prime}\right)$.

$-\operatorname{red}\left(f_{1}^{\prime \prime}\right)+\alpha_{1} \widehat{f} \subseteq \operatorname{red}\left(f_{1}^{\prime}\right)$.

$-\operatorname{red}\left(f_{2}^{\prime \prime}\right)+\alpha_{2} \widehat{f} \subseteq \operatorname{red}\left(f_{2}^{\prime}\right)$.

Portanto,

$$
\begin{aligned}
d-\operatorname{cl}(f) \widehat{f} & \in \operatorname{red}(t) \\
& \subseteq \operatorname{red}\left(f_{1}^{\prime \prime}\right)+\operatorname{red}\left(f_{2}^{\prime \prime}\right)+\operatorname{cl}(f) \widehat{f} \\
& \subseteq \operatorname{red}\left(f_{1}^{\prime}\right)+\operatorname{red}\left(f_{2}^{\prime}\right) \\
& \subseteq \operatorname{red}\left(f_{1}\right)+\operatorname{red}\left(f_{2}\right)
\end{aligned}
$$

Donde conclui-se que

$$
d \in \operatorname{red}\left(f_{1}\right)+\operatorname{red}\left(f_{2}\right)
$$

como queríamos.

O exemplo a seguir mostra que a inclusão reversa da segunda propriedade da proposição acima é falsa. Apresentamos ainda um exemplo que mostra que podem existir elementos $f$ e $g$, de modo que ambos se reduzem para zero sobre um conjunto $S$, mas sua soma não se reduz para zero sobre esse mesmo conjunto.

Exemplo 4.1.4 Tomamos um alfabeto $X=\{a, b, c\}$ e consideramos $K\langle X\rangle$. Fixamos ainda a ordem de grau e lexicografia em $X^{*}$ com a seguinte ordem nas letras de $X$ : $a>b>c$.

Com isso, se tomarmos $S=\left\{a^{2}-b, a^{2}-c\right\}, g_{1}=a^{2}$ e $g_{2}=-a^{2}$, teremos:

$$
\begin{aligned}
& \operatorname{red}\left(g_{1}\right)=\{b, c\} \\
& \operatorname{red}\left(g_{2}\right)=\{-b,-c\} \\
& \operatorname{red}\left(g_{1}+g_{2}\right)=\operatorname{red}(0)=\{0\} \subsetneq \operatorname{red}\left(g_{1}\right)+\operatorname{red}\left(g_{2}\right)=\{0, c-b, b-c\}
\end{aligned}
$$

Ainda, se tomarmos $H=\left\{a^{2}-b, a^{2}-c\right\}, f_{1}=a^{2}-b$ e $f_{2}=-a^{2}+c$, teremos que 0 pertence a red $\left(f_{1}\right)$ e a $\operatorname{red}\left(f_{2}\right)$ trivialmente. Porém, $\operatorname{red}\left(f_{1}+f_{2}\right)=\{-b+c\}$. 
Encerramos a seção com um exemplo que ilustra a necessidade da presença de uma boa ordem para que o Algoritmo da Divisão sempre termine sua computação em um número finito de passos, para qualquer entrada.

Exemplo 4.1.5 Considere $K\langle a, b\rangle$. Então teremos $\{a, b\}^{*}$ uma base multiplicativa. Tome a ordem lexicográfica com $a>b$ nessa base. Note que esta ordem não é admissível; nosso propósito é apresentar um exemplo em que o Algoritmo da Divisão não termine sua computação e isso só será possível devido ao fato de a ordem lexicográfica não ser uma boa ordem em $K\langle a, b\rangle$.

Considere então $S=\left\{f_{1}:=a^{2}+b a^{2}\right\}$ (note que $\widehat{f}_{1}=a^{2}$ na ordem lexicográfica) e $f=b a^{2}$. Seguem os valores atribuídos a $g$ nas primeiras iterações:

Primeira iteração: $g \leftarrow g-b f_{1}=-b^{2} a^{2}$

Segunda iteração: $g \leftarrow g+b^{2} f_{1}=b^{3} a^{2}$

Terceira iteração: $g \leftarrow g-b^{3} f_{1}=-b^{4} a^{2}$

Não é difícil verificar que esta computação continuará indefinidamente. Note que o conjunto dos termos líderes de $g$ a cada iteração $\left(\left\{b a^{2}, b^{2} a^{2}, b^{3} a^{2}, b^{4} a^{2}, \ldots\right\}\right)$ forma uma cadeia descendente infinita na ordem lexicográfica.

\subsubsection{O Algoritmo da Divisão em Álgebras de Caminhos}

Nesta seção tecemos alguns comentários breves sobre condições e modificações que são necessárias para que o algoritmo DIVISÃo descrito na seção anterior (Algoritmo 4.1.1) seja implementado para o contexto das álgebras de caminhos. Considere então $R=K \Gamma$ uma álgebra de caminhos.

Um primeiro ponto a ser considerado é a representação no computador dos próprios elementos de $K \Gamma$, bem como as operações que podem ser realizadas entre eles.

Para tanto, note primeiramente que, se temos o grafo $\Gamma$, é possível encontrar a forma canônica de um caminho qualquer: basta percorrer o grafo $\Gamma$ e armazenar a seqüência de arcos do caminho descrito. Ainda, podemos comparar facilmente dois monômios e é claro que a multiplicação entre monômios pode ser feita por concatenação, caso o passeio correspondente ao produto exista em $\Gamma$.

Com isso, podemos utilizar técnicas semelhantes às utilizadas para polinômios com o intuito de representar elementos de $K \Gamma$ em um computador. Algumas dessas técnicas são descritas na literatura [GCL92] e não é nossa intenção explorá-las aqui em profundidade. Mencionamos somente de forma muito resumida uma alternativa de implementação para o leitor não interessado em maiores detalhes (e nem em outras alternativas mais eficientes). Esses podem ser vistos em pacotes que implementam os algoritmos da Teoria de Bases de Gröbner e nos Capítulos 3 e 4 de [GCL92].

Tome $f$ em $K \Gamma$ e fixe a base $\mathcal{P}$ de $K \Gamma$. Então é claro que $f=\sum_{i=1}^{n} \alpha_{i} b_{i}$, com $\alpha_{i}$ escalares e $b_{i}$ monômios, para $1 \leq i \leq n$. Se assumirmos que todos os termos desta soma estão agrupados, podemos representar $f$ por uma lista ligada da seguinte maneira. Cada nó da lista consiste de um monômio $b$ de $\operatorname{supp}(f)$, o coeficiente (não nulo) de $b$ em $f$ e um ponteiro para o próximo nó. Os nós devem ser armazenados de forma ordenada na lista (de acordo, por exemplo, com a ordem admissivel fixada). ${ }^{7}$

\footnotetext{
${ }^{7} \mathrm{Na}$ realidade, o fato de armazenarmos um coeficiente em cada nó da lista nos remete ao problema de como representar elementos de $K$ no computador. Contudo, esse tipo de representaçāo já foi bem explorada na literatura, de forma que não a abordaremos aqui.
} 
Assim, é possível somar elementos de $K \Gamma$ e multiplicá-los por escalares ou monômios, percorrendo as listas que os representam. Ainda, dois elementos de $K \Gamma$ serão iguais se e somente se as listas ligadas que os representam forem iguais e um elemento será zero se e somente se for representado por uma lista vazia.

Destacamos a seguir os principais pontos adicionais que são necessários para uma implementação do Algoritmo da Divisão para as álgebras de caminhos.

O algoritmo deve ser capaz de encontrar o termo líder de um elemento $f$ de $R$. Isso pode ser obtido facilmente através da ordenação dos elementos do suporte de $f$, desde que seja possível comparar monômios algoritmicamente. Note que essa ordenação também foi utilizada acima, ao representar $f$ como uma lista ligada.

Diremos então que a ordem admissível fixada é computável se existe um algoritmo que recebe dois monômios como entrada e decide qual é menor. No que segue, assumiremos que dispomos de um tal algoritmo. Como exemplos de ordens admissíveis e computáveis, podemos citar todas as definidas na Seção 3.1 (deglex, weight-lex, rev-weight-lex e comut-lex).

Ademais, dividir um monômio por outro deve ser computável. Mais precisamente, dados monômios $u$ e $v$, deve existir uma função que ou devolve monômios $b$ e $b^{\prime}$ tais que $u=b v b^{\prime}$ ou diz que $v$ nâo divide $u$. Note que $b$ ou $b^{\prime}$ podem ser vértices de $\Gamma$ nos casos em que $v$ é um prefixo de $u$, um sufixo de $u$ ou igual a $u$.

Caso seja desejável tornar a descrição do algoritmo determinística, pode-se assumir que a função sempre devolve a divisão "mais à esquerda" possível. Mais precisamente, se considerarmos o conjunto $C$ de $\mathcal{B} \times \mathcal{B}$ dado por $C=\left\{\left(a, a^{\prime}\right) \in \mathcal{B} \times \mathcal{B} \mid u=a v a^{\prime}\right\}$, a função devolve sempre um par $\left(b, b^{\prime}\right)$ em $C$ tal que o comprimento de $b$ é minimal dentre os comprimentos de todos os elementos $a$, com $\left(a, a^{\prime}\right)$ em $C$.

Uma função como a descrita acima pode ser facilmente implementada fazendo uso de buscas por subpalavras, assumindo que $u$ e $v$ estão escritos na forma canônica.

O corpo $K$ deve ser computável, isto é, devem existir algoritmos que calculam as operações de corpos envolvendo elementos de $K$. Exemplos de corpos computáveis são $\mathbb{Z}_{p}$ e $\mathbb{Q}$.

Finalmente, caso seja desejável que a descrição do algoritmo seja determinística, pode-se assumir ainda que o conjunto de entrada $S$ é uma lista ordenada de elementos. Por outro lado, observamos que no restante do texto as aplicações do Algoritmo da Divisão serão independentes de qualquer escolha de divisores feita na linha 4 , de forma que qualquer implementação dessas escolhas (determinística ou não) é suficiente para nossos propósitos.

Como conseqüência da discussão acima, podemos enunciar o seguinte resultado.

Proposição 4.1.13 Dados um corpo $K$ e uma ordem admissivel $\leq$ computáveis, existe uma implementação efetiva do Algoritmo da Divisão em $K \Gamma$.

\subsection{Fundamentos Algébricos do Procedimento de Mora}

Esta seção tem o intuito de desenvolver algumas ferramentas algébricas que serão essenciais para a apresentação do Procedimento de Mora (que será visto na Seção 4.3). Mais especificamente, esta seção se dedica à demonstração do Teorema 4.2 .31 e a explorar algumas de suas consequièncias, limitações e ligações com outras áreas.

Conforme será explicitado adiante, esse teorema fornece $u m$ teste algorítmico para decidir se um conjunto gerador (finito) de um ideal $I$ de uma álgebra de caminhos é uma Base de Gröbner para 
I. Esse teste é um elemento chave para a elaboração do Procedimento de Mora.

Uma demonstração para o Teorema 4.2 .31 foi dada por Green [Gre97], mas a apresentada aqui é uma generalização feita por nós da demonstração de Mora para as álgebras livres [Mor94].

A Seção 4.2.1 retoma o conceito de elemento uniforme visto anteriormente e apresenta algumas de suas propriedades ainda não mencionadas neste trabalho. Esse conceito será utilizado livremente em todo o restante do texto. Em seguida, apresentamos o desenvolvimento das principais ferramentas algébricas para a demonstração do teorema na Seções 4.2.2 e 4.2.3. A Seção 4.2.4 resume os principais resultados obtidos pelo uso dessas ferramentas e apresenta um exemplo de aplicação do Teorema 4.2.31, mostrando que um dado conjunto constitui uma Base de Gröbner. Ainda, a Seção 4.2.5 apresenta um exemplo de um contexto mais geral que as álgebras de caminhos em que o Teorema 4.2.31 não é válido (ao menos, sem modificações em seu enunciado).

Antes de explorar as propriedades da álgebra de caminhos que nos levam a esse resultado, vale comentar que o teorema mencionado pode ser visto como uma reformulação do Lema do Diamante de Bergman [Ber78]. Com isso, na Seção 4.2.6, apresentamos brevemente alguns comentários sobre como esse importante resultado pode ser utilizado para demonstrar o Teorema 4.2.31. O único cuidado que temos nesta parte é o de utilizar o contexto das álgebras livres, dado que o Lema do Diamante foi originalmente enunciado para essas álgebras.

Assim, o leitor disposto a assumir o Lema do Diamante como conhecido (e a se limitar às álgebras livres) pode se restringir aos comentários desta seção. Por outro lado, esses comentários não têm a pretensão de introduzir os conceitos envolvidos com o Lema do Diamante, e podem ser deixados de lado pelo leitor não familiarizado com ele. ${ }^{8}$ De fato, a demonstração vista nas Seções 4.2 .1 a 4.2 .4 independe do Lema do Diamante (na realidade, ela contém em si muitos dos conceitos envolvidos na demonstração desse), além de abordar o caso mais geral das álgebras de caminhos.

Finalmente, na Seção 4.2.7, comentaremos brevemente sobre como encontrar exemplos de bases multiplicativas que não admitem nenhuma ordem admissível. No desenvolvimento da seção, faremos uso dos principais resultados apresentados nas seções anteriores, de modo que ela pode ser vista como uma aplicação do Algoritmo da Divisão e do Teorema 4.2.31.

\subsubsection{Uniformidade}

Relembramos aqui o conceito de elemento uniforme apresentado na Seção 3.4 .1 e exploramos algumas de suas propriedades básicas. Esse conceito não se faz presente em abordagens da Teoria de Bases de Gröbner no contexto das álgebras livres (com efeito, a partir da definição é imediato ver que em $K\langle X\rangle$ todo elemento é uniforme). Contudo, ele é fundamental para a generalização da teoria para as álgebras de caminhos.

Denotemos por $R$ uma álgebra qualquer que admita teoria de Bases de Gröbner. Relembramos a Definição 3.4.4 para conveniència do leitor. Umm elemento não nulo $f$ de $R$ é uniforme quando existem idempotentes $o$ e $t$ de $\mathcal{B}$, de forma que o $(b)=o$ e $t(b)=t$, para todo $b \operatorname{em} \operatorname{supp}(f)$.

O seguinte resultado é consequiencia imediata da definição de elemento uniforme e das propriedades dos idempotentes de $\mathcal{B}$ destacadas na Proposição 3.4.2 e no Corolário 3.4.5.

Corolário 4.2.1 Seja $R$ uma álgebra que admite teoria de Bases de Gröbner e $\mathcal{B}$ uma base multiplicativa de $R$ fixada. Então um elemento não nulo $f$ de $R$, cujo suporte é dado por $\operatorname{supp}(f)=$

\footnotetext{
${ }^{8}$ Contudo, o leitor interessado encontra no trabalho original de Bergman todas as definições e conceitos aqui mencionados. Outra boa introdução ao assunto é dada por Ferreira [Fer95].
} 
$\left\{b_{1}, b_{2}, \ldots, b_{k}\right\}$, é uniforme se e somente se para cada par $b, b^{\prime}$ de elementos idempotentes de $\mathcal{B}$, $b b_{i} b^{\prime}=0$ para todo $1 \leq i \leq k$ ou. $b b_{i} b^{\prime} \neq 0$ para todo $1 \leq i \leq k$.

\section{Demonstração.}

$(\Rightarrow)$

Tomemos um elemento $f$ uniforme de $R$. Denote por $o$ a origem dos elementos de $\operatorname{supp}(f)$ e por $t$ o término desses elementos. Então a Proposição 3.4.2 e o Corolário 3.4.5 nos mostram que para cada par $\left(b, b^{\prime}\right)$ de idempotentes de $\mathcal{B}$ diferente de $(o, t), b b_{i} b^{\prime}=0$, para todo $1 \leq i \leq k$. Além disso, $o b_{i} t \neq 0$, para todo $1 \leq i \leq k$.

$(\Leftarrow)$

Tome $f$ nas condições do enunciado do corolário e suponha por absurdo que $f$ não é uniforme. Então existem $b$ e $b^{\prime}$ no suporte de $f$, de modo que o $(b) \neq \mathrm{o}\left(b^{\prime}\right)$ ou $\mathrm{t}(b) \neq \mathrm{t}\left(b^{\prime}\right)$. Mostraremos o resultado na primeira situação.

Mas então o $\left(b^{\prime}\right) b \mathrm{t}\left(b^{\prime}\right)=0$ e o $\left(b^{\prime}\right) b^{\prime} \mathrm{t}\left(b^{\prime}\right) \neq 0$ pela Proposição 3.4 .2 e pelo Corolário 3.4.5 e chegamos a uma contradição com as hipóteses.

É importante notar que estaremos primordialmente interessados em utilizar o conceito de elemento uniforme no contexto das álgebras de caminhos. Nesse caso, podemos destacar a seguinte conseqüência do corolário acima.

Corolário 4.2.2 Sejam $R=K \Gamma$ uma álgebra de caminhos e $f$ um elemento não nulo de $R$, cujo suporte é dado por $\operatorname{supp}(f)=\left\{b_{1}, b_{2}, \ldots, b_{k}\right\}$. Então $f$ é uniforme se e somente se para cada $b, b^{\prime}$ em $\mathcal{P}, b b_{i} b^{\prime}=0$ para todo $1 \leq i \leq k$ ou $b b_{i} b^{\prime} \neq 0$ para todo $1 \leq i \leq k$.

Demonstração. O resultado segue imediatamente do Corolário 4.2.1 e do fato de que, em $K \Gamma, p q$ é não nulo se e somente se o $(q)=\mathrm{t}(p)$.

Uma conseqüência de grande importância desse último corolário é o fato de que, se multiplicarmos um elemento uniforme $f$ de $K \Gamma$ por um monômio $b$, então ou o resultado será zero, ou nenhuma multiplicação entre $b$ e elementos de $\operatorname{supp}(f)$ será igual a zero.

As duas propriedades destacadas a seguir são conseqüências imediatas da definição de elementos uniformes e serão utilizadas recorrentemente nas demonstrações adiante.

Lema 4.2.3 Seja g um elemento não nulo uniforme de $R=K \Gamma$. Então, se $l, r$ pertencem a $\mathcal{P}$, $\widehat{l g r}=l \widehat{g} r$, se lgr for não nulo. Conseqüentemente, $\lg r=0$ se e somente se l $\widehat{g} r=0$.

Lema 4.2.4 Seja g um elemento não nulo uniforme de $R=K \Gamma$. Então, existem monômios $b$ e $b^{\prime}$, tais que $g=b g b^{\prime}$.

Também vale ressaltar uma propriedade interessante dos ideais de álgebra admitindo teoria de Bases de Gröbner que nos mostra que podemos assumir sempre, sem nenhuma perda de generalidade, que um conjunto gerador de un ideal é composto apenas por elementos uniformes.

Proposição 4.2.5 Seja $R$ uma álgebra que admite teoria de Bases de Gröbner. Então todo ideal de $R$ pode ser gerado por elementos uniformes. Ademais, todo ideal finitamente gerado de $R$ possui um conjunto gerador finito composto apenas por elementos uniformes. 
Demonstração. Tomemos uma base multiplicativa $\mathcal{B}$ de $R$. Pelo Lema 3.4.1, a unidade de $R$ se escreve como combinação linear de elementos idempotentes:

$$
1=\sum_{j=1}^{n} \lambda_{j} v_{j}
$$

Tomemos um ideal $I=\left\langle g_{1}, g_{2}, \ldots\right\rangle$ de $R$. Então, pela Proposição 3.4.2, cada $g_{i}$ pode ser escrito como soma (finita) de elementos uniformes pertencentes a $I$ :

$$
g_{i}=1 g_{i} 1=\sum_{j=1}^{n} \sum_{k=1}^{n} \lambda_{j} \lambda_{k} v_{j} g_{i} v_{k}
$$

Claro que cada somando da expressão acima pertence a $I$ e é uniforme.

Lembramos que o Lema 3.4.10 nos mostrou que todo ideal 2-nomial pode ser gerado por um conjunto 2-nomial e uniforme.

Por outro lado, o exemplo a seguir nos mostra que nem todo ideal puramente binomial pode ser gerado por um conjunto puramente binomial e uniforme.

Exemplo 4.2.1 Considere o grafo $\Gamma$ dado na figura abaixo.

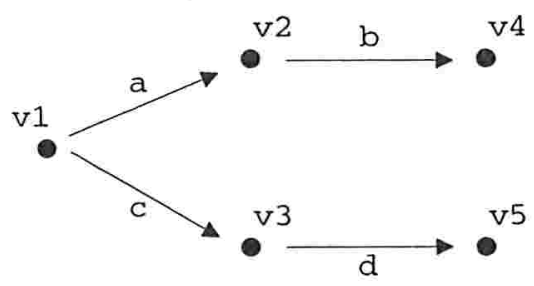

Com uma simples inspeção da figura acima, vemos que não existem caminhos paralelos em $\Gamma$. Portanto, os únicos elementos uniformes de $\mathcal{P}$ são os monômios. Assim, se tomarmos o ideal $I:=\langle a b-c d\rangle$, vemos que $I$ é puramente binomial, mas não possui um conjunto puramente binomial e uniforme.

Entretanto, note que $\{a b, c d\}$ é um conjunto gerador 2-nomial uniforme para $I$. $\Delta$

Continuamos com mais alguns lemas que serão úteis adiante.

Lema 4.2.6 Seja $f$ um elemento de $R=K \Gamma$ e b e $b^{\prime}$ dois monômios. Então bfb' é uniforme, se for não nulo.

Demonstração. Seja $f=\sum_{i=i}^{k} \alpha_{i} b_{i}$, com $\alpha_{i}$ escalares não nulos e $b_{i}$ em $\mathcal{P}, 1 \leq i \leq k$. Então, $b f b^{\prime}=\sum_{i=1}^{k} \alpha_{i} b b_{i} b^{\prime}$.

Claro que os únicos elementos $b b_{i} b^{\prime}$ não nulos serão aqueles que satisfazem: $\mathrm{o}\left(b_{i}\right)=\mathrm{t}(b)$ e $\mathrm{t}\left(b_{i}\right)=\mathrm{o}\left(b^{\prime}\right)$. Mas então, a origem de todos os elementos do suporte de $b f b^{\prime}$ será o $(b)$ e o término de todos esses elementos será $t\left(b^{\prime}\right)$. 
Lema 4.2.7 Sejam $f_{1}$ e $f_{2}$ dois elementos uniformes de $R$, tais que $\operatorname{supp}\left(f_{1}\right) \cap \operatorname{supp}\left(f_{2}\right) \neq \emptyset$. Então $\operatorname{supp}\left(f_{1}\right) \cup \operatorname{supp}\left(f_{2}\right)$ é um conjunto de elementos com mesma origem e mesmo término. Em particular, $f_{1}+\alpha f_{2}$ é uniforme, para todo $\alpha \in K$, se for não nulo.

Lema 4.2.8 Seja $S$ um subconjunto de $R=K \Gamma$ e $f$ um elemento uniforme tal que $f \Rightarrow_{S} f^{\prime}$. Então $f^{\prime}$ é um elemento uniforme, se for não nulo.

Demonstração. Para ver esse resultado, suponha que o Algoritmo da Divisão tenha recebido um elemento uniforme como entrada. Nessas condições, ele possui o seguinte invariante:

Invariante Inv No início de cada execução da linha 3 do algoritmo, os elementos $g$ e $h$ são nulos ou são uniformes. Ainda, $\operatorname{supp}(g) \cup \operatorname{supp}(h)$ é um conjunto de elementos com mesma origem e mesmo término.

O invariante é válido trivialmente no início da primeira execução, uma vez que $h=0$ e $g=f$ é uniforme. Suponha agora que o invariante é válido no início da $i$-ésima execução da linha 3 (e que não se trata da última execução dessa linha) e vejamos que ele se mantém válido no início da $(i+1)$-ésima execução dessa linha.

Considere então a $i$-ésima execução da linha 3 do processo. A partir deste ponto, a redução simples da linha 5 pode ser executada algumas vezes. Mas, pelos Lemas 4.2.6 e 4.2.7, segue que todos os elementos de $\operatorname{supp}(g) \cup \operatorname{supp}\left(b f_{i} b^{\prime}\right)$ têm mesma origem e mesmo término. Logo, $g-\frac{c l(g)}{c l\left(f_{i}\right)} b f_{i} b^{\prime}$ é um elemento uniforme e todos os elementos de $\operatorname{supp}\left(g-\frac{c l(g)}{c l\left(f_{i}\right)} b f_{i} b^{\prime}\right) \cup \operatorname{supp}(h)$ têm mesma origem e mesmo término, de forma que o invariante continua válido após a término do laço das linhas 4 e 5 .

Com isso, no início da execução da linha 6 (durante a $i$-ésima iteração do laço das linhas 3 a 8), os elementos dos suportes de $g$ e $h$ terão todos mesma origem e mesmo término. Não é difícil ver então, que o invariante continuará válido caso as linhas 7 e 8 sejam executadas. Com isso, o invariante continua válido no início da $(i+1)$-ésima execução da linha 3 do processo.

Claro que, em particular, o invariante é válido no início da última execução da linha 3 do processo, donde segue que a saída do Algoritmo da Divisão será um elemento uniforme, se for não nula.

\subsubsection{Resultados Principais}

Para o restante da seção, considere $R=K \Gamma$ uma álgebra de caminhos e sua base multiplicativa formada pelo conjunto de todos os caminhos em $\Gamma$ (denotado por $\mathcal{P}$ ). Como sempre, assumiremos que $K \Gamma$ é finitamente gerada e que temos uma ordem admissível fixada na base $\mathcal{P}$.

No que segue, tomaremos um conjunto gerador $G$ (possivelmente infinito, mas enumerável) de um ideal $I$ de $K \Gamma$. Podemos então dar uma ordem nos elementos de $G$ e, a partir disso, considerá-lo como um conjunto ordenado. Essa ordenação será essencial para formalização dos argumentos que seguem. 
Tome assim um conjunto de índices $\mathcal{I}$, definido como $\mathcal{I}:=\mathbb{N}$, no caso em que $G$ é infinito ou $\mathcal{I}:=\{x \in \mathbb{N} \mid x \in[0, n]\}$, no caso que que $G$ é finito $(\operatorname{com} \operatorname{card}(G)=n)$. Considere então

$$
G=\left(g_{i}\right)_{i \in \mathcal{I}}
$$

Assuma que os elementos de $G$ são uniformes. Nosso objetivo é desenvolver um teste para decidir se $G$ é uma Base de Gröbner para $I$. Posteriormente, veremos que esse teste é a peça chave do Procedimento de Mora.

Um ponto fundamental para desenvolver o teste em que estamos interessados é estudar certos múltiplos de elementos de $G$, isto é, elementos da forma $\lg _{i} r$, com $l, r$ caminhos. Para tanto, vamos considerar o conjunto $(\mathcal{P} \cup\{0\}) \times(\mathcal{P} \cup\{0\})$. Temos nesse conjunto algumas operações interessantes. Em particular, podemos definir uma multiplicação à esquerda e à direita por $\mathcal{P} \cup\{0\}$. Com efeito, dados $(l, t) \in(\mathcal{P} \cup\{0\}) \times(\mathcal{P} \cup\{0\})$ e $p \in \mathcal{P} \cup\{0\}$, teremos:

$$
(l, t) p:=(l, t p) \text { e } p(l, t):=(p l, t)
$$

Trabalharemos ainda com a noção de ideal em $(\mathcal{P} \cup\{0\}) \times(\mathcal{P} \cup\{0\})$. Diremos que um subconjunto $J$ de $(\mathcal{P} \cup\{0\}) \times(\mathcal{P} \cup\{0\})$ é um ideal se sempre que $(l, r)$ pertencer a $J$ e $t$ pertencer a $\mathcal{P} \cup\{0\}$, os elementos $(t l, r)$ e $(l, r t)$ também pertencerem a $J$.

Ao longo de toda esta seção trabalharemos com ideais da base multiplicativa $\mathcal{P}$. Assim, se $A$ for um subconjunto de $\mathcal{P}$, o ideal de semigrupo gerado por $A$ será denotado por $\langle A\rangle_{\mathcal{P}}$ e a notação convencional continuará denotando ideais de álgebra. ${ }^{9}$

Em seguida consideramos o seguinte conjunto gerador de $I$ (como espaço vetorial):

$$
\mathcal{G}:=\{l g r \mid l g r \neq 0, g \in G \text { e }(l, r) \in(\mathcal{P} \cup\{0\}) \times(\mathcal{P} \cup\{0\})\}
$$

Se definimos

$$
T=\{(l, r, g) \in(\mathcal{P} \cup\{0\}) \times(\mathcal{P} \cup\{0\}) \times G \mid l g r \neq 0\}
$$

então

$$
\mathcal{G}=\left(\cup_{(l, r, g) \in T} l g r\right)
$$

Note que como os elementos de $G$ são uniformes, $\widehat{\mathcal{G}}=\langle\widehat{G}\rangle_{\mathcal{P}} \backslash\{0\}$.

Vamos dar uma boa ordem nos elementos de $T$. Diremos que $\left(l_{1}, r_{1}, g_{j_{1}}\right)<_{T}\left(l_{2}, r_{2}, g_{j_{2}}\right)$ se

$l_{1} \widehat{g_{1}} r_{1}<l_{2} \widehat{g_{j_{2}}} r_{2}($ de acordo com a ordem admissível fixada em $\mathcal{P})$ ou

$l_{1} \widehat{g_{j_{1}}} r_{1}=l_{2} \widehat{g_{2}} r_{2}$ e $j_{1}<j_{2}$ ou

$l_{1} \widehat{g_{j_{1}}} r_{1}=l_{2} \widehat{g_{j_{2}}} r_{2}$ e $j_{1}=j_{2}$ e $r_{2}=w r_{1}$, com $r_{2} \neq r_{1}$ e $w \in \mathcal{P}$ (isto é, $w$ é um caminho de comprimento maior que zero).

Uma situação em que ocorre o terceiro caso é ilustrada no diagrama a seguir:

\footnotetext{
${ }^{9}$ Deve estar claro que $\langle A\rangle_{P}$ é um ideal de semigrupo de $\mathcal{P} \cup\{0\}$.
} 


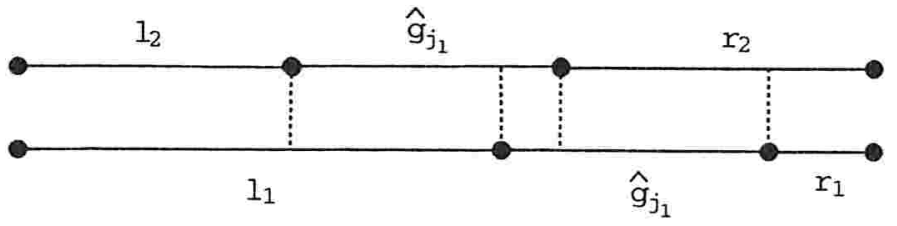

No diagrama, $l_{1} \widehat{g_{j_{1}}} r_{1}=l_{2} \widehat{g_{j_{1}}} r_{2}$. Entretanto, como $r_{2}=w r_{1}$, para algum $w$ de comprimento maior que zero, segue que $\left(l_{1}, r_{1}, g_{j_{1}}\right)<_{T}\left(l_{2}, r_{2}, g_{j_{1}}\right)$.

Em seguida, mostramos que esta ordem definida em $T$ é uma boa ordem. Antes, entretanto, apresentamos um resultado técnico de verificação imediata.

Lema 4.2.9 Sejam $\left(l_{1}, r_{1}, g\right)$ e $\left(l_{2}, r_{2}, g\right)$ dois elementos de $T$ satisfazendo $l_{1} \widehat{g} r_{1}=l_{2} \widehat{g} r_{2}$. Então as seguintes implicações são verdadeiras.

- Se o comprimento de $r_{1}$ é igual ao comprimento de $r_{2}$ então $r_{1}=r_{2}$ e $l_{1}=l_{2}$, donde $\left(l_{1}, r_{1}, g\right)=\left(l_{2}, r_{2}, g\right)$.

- Se o comprimento de $l_{1}$ é igual ao comprimento de $l_{2}$ então $r_{1}=r_{2}$ e $l_{1}=l_{2}$, donde $\left(l_{1}, r_{1}, g\right)=$ $\left(l_{2}, r_{2}, g\right)$.

Proposição 4.2.10 A ordem $<_{T}$ é uma boa ordem satisfazendo: se $\left(l_{1}, r_{1}, g_{j_{1}}\right)<_{T}\left(l_{2}, r_{2}, g_{j_{2}}\right)$ e $(l, r) \in(\mathcal{P} \cup\{0\}) \times(\mathcal{P} \cup\{0\})$, então $\left(l l_{1}, r_{1} r, g_{j_{1}}\right)<_{T}\left(l l_{2}, r_{2} r, g_{j_{2}}\right)$ se ambos forem elementos de $T$.

Demonstração. Vejamos primeiro que se trata de uma boa ordem. Tomemos então $X$ um subconjunto qualquer de $T$ e mostraremos que ele possui um menor elemento. Mas então considere o subconjunto $A$ de $\mathcal{P}$, dado por $A:=\left\{l \widehat{g_{i}} r \mid\left(l, r, g_{i}\right) \in X\right\}$. Como $\mathcal{P}$ é um conjunto bem ordenado (pela ordem admissível fixada), podemos encontrar um menor elemento de $A$ : denotemo-lo por $m$.

Considere então $X_{1}$ o conjunto formado por todos os elementos $(l, r, g)$ de $X$ tais que $l \widehat{g} r=m$. Claro que todos os elementos de $X \backslash X_{1}$ são estritamente maiores que cada um dos elementos de $X_{1}$, pela definição de $<_{T}$. Ainda, $X_{1}$ é composto por elementos da forma $\left(l, r, g_{j}\right)$, tais que $l \widehat{g_{j}} r=m$.

Podemos então definir o subconjunto $X_{2}$ de $X_{1}$ composto pelos elementos de $X_{1}$ com $j$ mínimo. Com isso, todos os elementos de $X_{1} \backslash X_{2}$ serão estritamente maiores que os elementos de $X_{2}$. Portanto, se mostrarmos que $X_{2}$ possui um menor elemento, esse certamente será o menor elemento de $X$.

Note que cada elemento de $X_{2}$ é da forma $\left(l, r, g_{j}\right)$ e satisfaz $l \widehat{g_{j}} r=m$. Logo, pelo Lema 4.2 .9 , podemos assumir que, dados dois elementos distintos $\left(l_{1}, r_{1}, g_{j}\right)$ e $\left(l_{2}, r_{2}, g_{j}\right)$ de $X_{2}$, os comprimentos de $r_{1}$ e $r_{2}$ são distintos. Assim, podemos encontrar um elemento $\left(l, r, g_{j}\right)$ de $X_{2}$ tal que $r$ possui comprimento mínimo dentre todos os elementos de $X_{2}$. Mas, pela definição de $<_{T},\left(l, r, g_{j}\right)$ será o menor elemento de $X_{2}$ e, portanto, de $X$, donde segue que $<_{T}$ é uma boa ordem.

A segunda parte é de verificação imediata. 
Para decidir se um conjunto $G$ é uma Base de Gröbner para $I:=\langle G\rangle$, precisamos verificar se os termos líderes de todos os elementos do ideal $I$ são divisíveis pelo termo líder de algum elemento de $G$. Mas por um lado, todo elemento de $I$ se escreve como uma soma de múltiplos de elementos de $G$ (mais precisamente, como uma combinação linear de elementos de $\mathcal{G}$ ).

Intuitivamente, podemos dizer que se nessa soma não ocorre nenhum cancelamento entre termos líderes de elementos de $\mathcal{G}$, então o termo líder da soma pertence a $\widehat{\mathcal{G}}$. Com isso, esse processo de cancelamento é fundamental para descobrirmos se um conjunto é ou não uma Base de Gröbner.

O leitor atento recordará de nossa abordagem do caso comutativo que a ferramenta utilizada para lidar com essa situação naquele caso foram os S-polinômios. Aqui, utilizaremos uma ferramenta muito parecida, mas vale mencionar que a situação se torna sensivelmente mais complexa. Em particular, veremos que será necessário considerar mais elementos que no caso comutativo.

Uma amostra desses comentários pode ser vista ao se considerar ideais principais. No caso comutativo, um gerador de um ideal principal sempre é uma Base de Gröbner para esse ideal (o único S-polinômio que pode ser produzido é igual a zero). O exemplo seguinte mostra que o mesmo não é verdade para o caso não comutativo.

Exemplo 4.2.2 Considere $K\langle a, b\rangle$, tome como base multiplicativa o conjunto de todas as palavras no alfabeto $\{a, b\}$ e fixe a ordem deglex com $a>b$. Se consideramos o elemento $g=a b a-b^{2}$, podemos ver que $g^{\prime}:=g b a-a b g=a b^{3}-b^{3} a$ pertence a $\langle g\rangle$. Porém , $\widehat{g^{\prime}}=a b^{3}$ claramente não é divisível por $a b a$.

Note que nesse exemplo o "novo" termo líder surgiu a partir de um cancelamento entre múltiplos de $a b a=\widehat{g}$.

Nosso objetivo é, portanto, encontrar elementos distintos com o mesmo termo líder em $I$. Eles são importantes porque causam cancelamento de termos líderes. De uma maneira muito geral e intuitiva, o que faremos nesta seção é considerar esses cancelamentos entre termos líderes. Assim, sempre que houver um cancelamento, podemos tentar reescrever o elemento como uma combinação linear de elementos de $\mathcal{G}$ formados a partir de triplas (elementos de $T$ ) menores que os que causaram o cancelamento (segundo $<_{T}$ ).

Se isso sempre é possível, então o processo de "reescrita" precisa terminar, dado que estamos lidando com uma boa ordem (em $T$ ). Mas quando o processo termina, temos uma combinação linear de elementos de $\mathcal{G}$ entre os quais não ocorre cancelamento de termos líderes; logo o termo líder da combinação precisa ser um múltiplo de um elemento de $G$.

O conjunto composto pelos elementos de $\mathcal{G}$ entre os quais não ocorre cancelamento de termos líderes será de importância fundamental para a formalização dos argumentos que seguem. Intuitivamente, utilizaremos o conceito de Base de Gauss para caracterizar um subconjunto de $\mathcal{G}$ entre cujos elementos não há cancelamento de termos líderes e que "contém" todos os termos líderes de elementos de $\mathcal{G}$. Na realidade, para construir tal conjunto, teremos de considerar todas as triplas distintas que dão origem a elementos de mesmo termo líder e escolher somente uma delas (escolheremos sempre as menores segundo $<_{T}$ ). Os conjuntos que são definidos a seguir têm exatamente esse propósito.

Apresentamos as definições de alguns conjuntos que servirão de base para o que será desenvolvido adiante. As definições aqui apresentadas são generalizações de definições apresentadas por Mora, de forma que optamos por manter a nomenclatura original para elas.

Primeiramente precisamos encontrar os pares de $(\mathcal{P} \cup\{0\}) \times(\mathcal{P} \cup\{0\})$ que anulam o termo líder de um elemento $g_{j}$ de $G$. Porém, como estamos assumindo que os elementos de $G$ são uniformes, 
isso é equivalente a encontrar os pares $(l, r)$ de $(\mathcal{P} \cup\{0\}) \times(\mathcal{P} \cup\{0\})$, tais que $l g_{j} r=0$. Definimos então o conjunto $\mathrm{Null}(j)$ :

$$
N u l l(j):=\left\{(l, r) \in(\mathcal{P} \cup\{0\}) \times(\mathcal{P} \cup\{0\}) \mid l g_{j} r=0\right\}
$$

Em seguida, encontramos, para cada $g_{j}$ de $G$, os elementos de $(\mathcal{P} \cup\{0\}) \times(\mathcal{P} \cup\{0\})$ que causam o fenômeno visto no Exemplo 4.2.2, isto é, cancelamento de termos líderes envolvendo dois múltiplos de um mesmo elemento $g_{j}{ }^{10}$

$$
S O B(j):=\left\{\left(\mathrm{o}\left(\widehat{g_{j}}\right), r\right) \in(\mathcal{P} \cup\{0\}) \times(\mathcal{P} \cup\{0\}) \mid r \neq \mathrm{t}\left(\widehat{g_{j}}\right) \text { e existe } l \in \mathcal{P} \cup\{0\} \text { tal que } l \widehat{g_{j}}=\widehat{g_{j}} r\right\}
$$

Ainda, para cada $g_{j} \in G$, consideramos o ideal de $(\mathcal{P} \cup\{0\}) \times(\mathcal{P} \cup\{0\})$ gerado por $\operatorname{SOB}(j)$ :

$$
S T(j):=\langle S O B(j)\rangle \text { (ideal de }(\mathcal{P} \cup\{0\}) \times(\mathcal{P} \cup\{0\}))
$$

Com isso, note que, para todo par $(l, r)$ em $S T(j)$, existe outro par $\left(l^{\prime}, r^{\prime}\right)$ em $(\mathcal{P} \cup\{0\}) \times(\mathcal{P} \cup\{0\})$, distinto de $(l, r)$, tal que $l \widehat{g_{j}} r=l^{\prime} \widehat{g}_{j} r^{\prime}$ (essa afirmação será demonstrada no Lema 4.2.14).

Uma observação importante é o fato de que $S T(j)$ pode ser infinitamente gerado como ideal de $(\mathcal{P} \cup\{0\}) \times(\mathcal{P} \cup\{0\})$. Registramos e mostramos esse fato no seguinte exemplo.

Exemplo 4.2.3 Se tomamos um elemento $g_{j}$ de $K\langle a, b\rangle$ com termo líder igual a $a b a$, então $\left(1, b^{i} a b a\right) \in S O B(j) \subseteq S T(j)$, para todo $i$.

Com o que temos até aqui, consideramos cancelamentos de termos líderes envolvendo múltiplos de um mesmo $g_{j}$ de $G$. Agora é necessário considerar o mesmo fenômeno envolvendo termos líderes de elementos distintos de $G$. Para tanto, definimos o conjunto $T(j)$, para cada $g_{j} \in G$ :

$$
T(j):=\left\{(l, r) \in(\mathcal{P} \cup\{0\}) \times(\mathcal{P} \cup\{0\}) \mid l \widehat{g_{j}} r \in\left\langle\widehat{g_{1}}, \widehat{g_{2}}, \ldots, \widehat{g_{j-1}}\right\rangle\right\} \cup S T(j)
$$

Se considerarmos as triplas $\left(l, r, g_{j}\right)$ de $T$ tais que $(l, r)$ não pertence a $T(j)$, teremos elementos com propriedades bastante interessantes, as quais serão exploradas adiante.

Retomando nossa discussão intuitiva dos parágrafos anteriores, o leitor deve se recordar que estamos interessados em construir um conjunto que contenha um elemento para cada termo líder que surge entre os elementos de $\mathcal{G}$ e que tenha somente elementos com termos líderes distintos. Os elementos destacados abaixo representam aqueles que foram "escolhidos" para fazer parte desse conjunto. Com isso, para trabalhar de maneira mais conveniente com esses elementos, definimos o conjunto $O(j)$.

$$
O(j):=((\mathcal{P} \cup\{0\}) \times(\mathcal{P} \cup\{0\})) \backslash(N u l l(j) \cup T(j))
$$

Ainda, por conveniência, definimos o seguinte subconjunto de $\widehat{\mathcal{G}}$ :

$$
E(j):=\left\{l \widehat{g_{j}} r \mid(l, r) \in O(j)\right\}
$$

\footnotetext{
${ }^{10} \mathrm{O}$ nome desse conjunto, $S O B(j)$, vem do termo em inglès self-obstruction.
} 
No que segue, apresentaremos uma série de lemas envolvendo os conjuntos aqui definidos. O entendimento de muitas das demonstrações não requer mais que uma boa compreensão das definições dadas, de forma que o leitor é convidado a examinar essas últimas com cuidado antes de prosseguir. Seguem alguns exemplos que ilustram as definições.

Exemplo 4.2.4 Considere $X=\{a, b, c\}$ e tome $K\langle X\rangle$, com $X^{*}$ como base multiplicativa e a ordem deglex com $a<b<c$. Seja então $G:=\left(g_{1}, g_{2}\right)$, onde $g_{1}=c b c-a^{2}$ e $g_{2}=c a-b^{2}$. Então:

$$
\begin{aligned}
& \operatorname{Null}(1)=\operatorname{Null}(2)=\left\{(l, 0) \mid l \in X^{*}\right\} \cup\left\{(0, r) \mid r \in X^{*}\right\} \\
& \text { SOB }(1)=\{(1, b c)\} \cup\left\{(1, w c b c) \mid w \in X^{*} \cup\{0\}\right\}
\end{aligned}
$$

$S T(1)$ é o conjunto formado pelos elementos de $\left(X^{*} \cup\{0\}\right) \times\left(X^{*} \cup\{0\}\right)$ da forma $(l, r)$, tais que $r$ é múltiplo à direita de $b c$ ou $r$ é múltiplo de $c b c$ (múltiplo em $\left.X^{*} \cup\{0\}\right)$

$T(1)=S T(1)$

$S O B(2)=\left\{\left(1, w c a \mid w \in X^{*} \cup\{0\}\right\}\right.$

$S T(2)$ é o conjunto formado pelos elementos de $\left(X^{*} \cup\{0\}\right) \times\left(X^{*} \cup\{0\}\right)$ da forma $(l, r)$, tais que $r$ é múltiplo de ca (em $\left.X^{*} \cup\{0\}\right)$

$T(2)$ é o conjunto formado pelos elementos $(l, r)$ de $\left(X^{*} \cup\{0\}\right) \times\left(X^{*} \cup\{0\}\right)$ satisfazendo:

$r$ é múltiplo de $c a$ ou

$l$ é múltiplo à esquerda de $c b$ ou

$r$ é múltiplo de $c b c$ ou

$l$ é múltiplo de $c b c$

Vejamos um exemplo para a álgebra de caminhos.

Exemplo 4.2.5 Considere o grafo $\Gamma$ dado na figura abaixo e a álgebra $K \Gamma$ com a ordem deglex tal que $a>b>\cdots>g$.

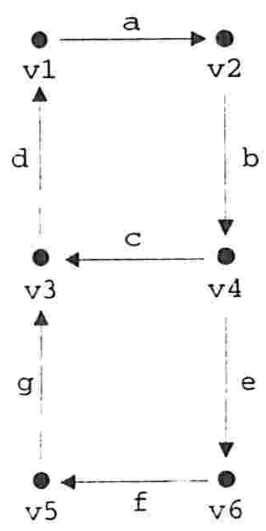

Seja $G:=\left(g_{1}, g_{2}\right)$, onde $g_{1}=c d a b c d a b-e f g d a b$ e $g_{2}=c d a b$. Então teremos: 
$\operatorname{Null}(1)$ é o conjunto dos elementos de $(\mathcal{P} \cup\{0\}) \times(\mathcal{P} \cup\{0\})$ da forma $(c, h)$ satisfazendo:

$c=0$ ou $h=0$ ou

$c \neq 0$ e $h \neq 0$ e $(c, h)$ é tal que $\mathrm{t}(c) \neq v_{4}$ ou o $(h) \neq v_{4}$

$S O B(1)=\left\{\left(v_{4}\right.\right.$, wcdabcdab $\left.) \mid w \in(\mathcal{P} \cup\{0\}) \times(\mathcal{P} \cup\{0\})\right\} \cup\left\{\left(v_{4}, c d a b\right)\right\}$

$S T$ (1) é o conjunto formado pelos elementos de $(\mathcal{P} \cup\{0\}) \times(\mathcal{P} \cup\{0\})$ da forma

$\left(l, w c d a b c d a b l^{\prime}\right)$ ou $\left(l, c d a b l^{\prime}\right), \operatorname{com} l, l^{\prime}, w$ em $(\mathcal{P} \cup\{0\}) \times(\mathcal{P} \cup\{0\})$.

$T(1)=S T(1)$

$\operatorname{Null}(2)=\operatorname{Null}(1)$

$S O B(2)=\left\{\left(v_{4}, w c d a b\right) \mid w \in(\mathcal{P} \cup\{0\}) \times(\mathcal{P} \cup\{0\})\right\}$

$S T(2)=\left\{\left(l, w c d a b l^{\prime}\right) \mid l, l^{\prime}, w \in(\mathcal{P} \cup\{0\}) \times(\mathcal{P} \cup\{0\})\right\}$

$T(2)$ é o conjunto formado pelos elementos de $(\mathcal{P} \cup\{0\}) \times(\mathcal{P} \cup\{0\})$ da forma

$(l, r)$ tais que

$r$ é múltiplo de $c d a b$ ou

$l$ ou $r$ é múltiplo de $c d a b c d a b$ ou

$l$ é múltiplo à esquerda de $c d a b$ ou $r$ é múltiplo à direita de $c d a b$.

Lema 4.2.11 O conjunto $T(j)$ é um ideal de $(\mathcal{P} \cup\{0\}) \times(\mathcal{P} \cup\{0\})$, para todo $j \in \mathcal{I}$.

Demonstração. Seja $p$ em $\mathcal{P} \cup\{0\}$ e $(l, r)$ em $T(j)$, para algum $1 \leq j \leq s$. Então, pela definição de $T(j),(l, r)$ pertence a $S T(j)$ (que é um ideal de $(\mathcal{P} \cup\{0\}) \times(\mathcal{P} \cup\{0\})$ ) ou $(l, r)$ é tal que $l \widehat{g_{j}} r \in\left\langle\widehat{g_{1}}, \widehat{g_{2}}, \ldots, \widehat{g_{j-1}}\right\rangle$. Em ambos os casos, teremos que $(p l, r)$ e $(l, r p)$ pertencem a $T(j)$, o que encerra a demonstração.

Lema 4.2.12 Para todos $i, j \in \mathcal{I}$, se existem $(l, r) \in O(i)$ e $\left(l^{\prime}, r^{\prime}\right) \in O(j)$ tais que $l \widehat{g}_{i} r=l^{\prime} \widehat{g_{j}} r^{\prime}$, então $\left(l^{\prime}, r^{\prime}\right)=(l, r)$ e $i=j$.

Demonstração. A demonstração desse resultado depende somente de um bom entendimento das definições dos conjuntos $O(i)$ e $O(j)$. Primeiramente, vale comentar que tanto $l \widehat{g_{i}} r$ quanto $l^{\prime} \widehat{g_{j}} r^{\prime}$ são não nulos, pelas definições de $O(i)$ e $O(j)$. Suponha então que o resultado seja falso e mostraremos que isso nos leva a uma contradição. Mas então, temos duas possibilidades: ou $i=j$ ou $i \neq j$.

Se $i=j$ e $(l, r)=\left(l^{\prime}, r^{\prime}\right)$ não há o que demonstrar. Assuma, portanto, que $(l, r) \neq\left(l^{\prime} . r^{\prime}\right)$. Mas então, pelo Lema 4.2.9, temos que os comprimentos de $r$ e de $r^{\prime}$ são diferentes. Supomos, sem perda de generalidade, que o comprimento de $r^{\prime}$ é menor que o de $r$.

Mas então existe um divisor de $l^{\prime}$ (à direita) $l_{1}$ e um divisor de $r$ (à esquerda) $r_{1}$, tais que $l_{1} \widehat{g_{j}}=\widehat{g_{j}} r_{1}$, donde $(l, r)$ pertence a $S T(j) \subseteq T(j)$, uma contradição.

Vejamos agora o caso em que $i \neq j$. Podemos supor, sem perda de generalidade, que $i<j$. Mas então, $l^{\prime} \widehat{g}_{j} r^{\prime}$ pertence a $\left\langle\widehat{g_{1}}, \widehat{g_{2}} \ldots, \widehat{g_{i}}, \ldots, \widehat{g_{j-1}}\right\rangle$ e concluímos que $\left(l^{\prime}, r^{\prime}\right)$ pertence a $T(j)$, um absurdo. 
Antes de continuarmos, definimos alguma notação. Seja $c$ um monômio e denote por $F_{c}$ o conjunto formado por todas as triplas $\left(l, r, g_{j}\right)$ de $T$ tais que $l \widehat{g_{j}} r=c$. Vale ressaltar que, da definição de $T$, decorre que, toda tripla $\left(l, r, g_{j}\right)$ em $F_{c}$ tem a propriedade de que $(l, r)$ não pertence a $\operatorname{Null}(j)$. Note ainda que, como os elementos de $T$ estão bem ordenados por $<_{T}$, cada conjunto não vazio $F_{c}$ possui um elemento mínimo.

Lema 4.2.13 Um elemento $\left(l, r, g_{j}\right)$ de $F_{c}$ é mínimo (com relação a $<_{T}$ ) em $F_{c}$ se e somente se $(l, r)$ pertence a $O(j)$. Em particular, existe $j$ tal que $F_{c} \cap O(j)$ é um conjunto unitário e $F_{c} \cap O(i)=\emptyset$, para todo $i$ distinto de $j$.

Demonstração. Tome $\left(l, r, g_{j}\right)$ em $F_{c}$ e assuma que ele é mínimo em $F_{c}$. Precisamos mostrar que $(l, r)$ não pertence a $T(j)$. Mas por um lado, se $l \widehat{g_{j}} r$ pertence a $\left\langle\widehat{g_{1}}, \widehat{g_{2}}, \ldots, \widehat{g_{j-1}}\right\rangle$, então teremos que $l \widehat{g_{j}} r=l^{\prime} \widehat{g_{i}} r^{\prime}$, para algum $i<j$, o que contraria a minimalidade de $\left(l, r, g_{j}\right) .{ }^{11}$

Por outro lado, se $(l, r)$ pertence a $S T(j)$, então existe $r_{1}$, tal que (o $\left.\left(\widehat{g_{j}}\right), r_{1}\right)$ pertence a $S O B(j)$ e $r=r_{1} r_{2}$, para algum $r_{2} \in \mathcal{P}$. Logo, pela definição de $S O B(j)$, existe $l_{1}$ tal que $l_{1} \widehat{g_{j}}=\widehat{g_{j}} r_{1} \neq 0$. Note que as multiplicações são não nulas pelo fato de $(l, r)$ não pertencer a $N u l l(j)$ e $r_{1}$ ser um divisor de $r$. Ainda, pela definição de $S O B(j),\left\|r_{1}\right\|>0$, donde $\left\|r_{2}\right\|<\|r\|$.

Logo, $l l_{1} \widehat{g_{j}} r_{2}=l \widehat{g_{j}} r_{1} r_{2}=l \widehat{g_{j}} r \neq 0$. Mas então, $\left(l l_{1}, r_{2}, g_{j}\right)$ pertence a $F_{c}$ e é menor (segundo $<_{T}$ ) que $\left(l, r, g_{j}\right)$ (pois $r_{2}$ divide propriamente $r$ à direita), contradizendo a minimalidade desse último elemento.

Para ver a implicação reversa, tome $\left(l, r, g_{j}\right)$ em $F_{c}$ tal que $(l, r)$ pertence a $O(j)$. Mostraremos que $\left(l, r, g_{j}\right)$ é o menor elemento de $F_{c}$. Suponha que isso não ocorre, ou seja, existe $\left(l^{\prime}, r^{\prime}, g_{i}\right)$ em $F_{c}$ menor que $\left(l, r, g_{j}\right)$. Logo, podemos concluir que $i=j$ pela minimalidade de $\left(l^{\prime}, r^{\prime}, g_{i}\right)$ e pelo fato de $(l, r)$ pertencer a $O(j)$. Então, somos levados a concluir que $r=w r^{\prime}$, para algum monômio $w$ e $\left(\mathrm{o}\left(\widehat{g_{j}}\right), w\right)$ pertence a $S O B(j)$, donde $(l, r)$ pertence a $S T(j)$, um absurdo.

Lema 4.2.14 Para todo $(l, r)$ em $T(j) \backslash N u l l(j)$, existe um elemento $\left(l^{\prime}, r^{\prime}\right)$ em $O(i)$, tal que $l^{\prime} \widehat{g}_{i} r^{\prime}=$ $l \widehat{g_{j}} r$ e $\left(l^{\prime}, r^{\prime}, g_{i}\right)<_{T}\left(l, r, g_{j}\right)$.

Demonstração. Se considerarmos $F_{l \widehat{g_{j}} r}$, vemos por hipótese que ele é não vazio. Mas, como se trata de um subconjunto de $T$, ele possui um menor elemento: $\left(l^{\prime}, r^{\prime}, g_{i}\right)$. Pelo Lema 4.2.13, esse menor elemento é tal que $\left(l^{\prime}, r^{\prime}\right)$ pertence a $O(i)$. Logo, $\left(l^{\prime}, r^{\prime}, g_{i}\right) \leq_{T}$ $\left(l, r, g_{j}\right)$, donde $i \leq j$. Se $i<j,\left(l^{\prime}, r^{\prime}, g_{i}\right)<_{T}\left(l, r, g_{j}\right)$ e segue a afirmação do lema. Se $i=j$, segue da definição dos conjuntos $O(i)$ e $T(j)$ que $(l, r)$ não pertence a $O(i)$, donde $\left(l, r, g_{j}\right) \neq\left(l^{\prime}, r^{\prime}, g_{i}\right)$ e o resultado está demonstrado.

Lema 4.2.15 O conjunto $\mathcal{P}$ é a união disjunta dos conjuntos: $(\mathcal{P} \backslash \widehat{\mathcal{G}})$ e $E(j)$, para todo $j \in \mathcal{I}$.

Demonstração. Para evitar maiores problemas, note que os elementos de cada $E(i)$, para $i \in \mathcal{I}$ são não nulos por definição. Em seguida, dado $i \in \mathcal{I}$, claro que $E(i) \subseteq \widehat{\mathcal{G}}$,

\footnotetext{
${ }^{11}$ Lembramos que como o ideal $\left\langle\widehat{g_{1}}, \widehat{g_{2}}, \ldots, \widehat{g_{j-1}}\right\rangle$ é monomial, todo monòmio pertencente a esse ideal é múltiplo de algum gerador.
} 
donde $E(i) \cap(\mathcal{P} \backslash \widehat{\mathcal{G}})=\emptyset$. Além disso, o Lema 4.2 .12 nos mostra que os conjuntos $E(i)$ $(1 \leq i \leq s)$ são dois a dois disjuntos. Resta, portanto mostrar que $\widehat{\mathcal{G}}$ é a união dos conjuntos $E(i), 1 \leq i \leq s$.

Para tanto, tomemos um monômio $c$ em $\widehat{\mathcal{G}}$ e consideremos $F_{c}$. Claro que $F_{c}$ é não vazio pelo fato de $c$ pertencer a $\widehat{\mathcal{G}}$. Então, pelo Lema 4.2 .13 , o menor elemento de $F_{c}$ (chamemo-no de $\left.\left(l, r, g_{i}\right)\right)$, é tal que $(l, r)$ pertence a $O(i)$, donde segue que $l \widehat{g_{i}} r=c$ pertence a $E(i)$, para algum $1 \leq i \leq s$.

Definimos em seguida $E(G):=\left\{l_{i} r \mid i \in \mathcal{I}\right.$ e $\left.(l, r) \in O(i)\right\}$.

O lema a seguir é conseqüência da definição de $E(G)$ e do Lema 4.2.15.

Lema 4.2.16 A seguinte igualdade é verdadeira:

$$
\widehat{E(G)}=\widehat{\mathcal{G}}=\bigcup_{i \in \mathcal{I}} E(i)
$$

O seguinte resultado é conseqüência imediata do Lema 4.2.13.

Lema 4.2.17 Cada elemento $\lg _{j} r$ de $E(G)$ é tal que $\left(l, r, g_{j}\right)$ é mínimo dentre os elementos de $F_{l \widehat{g_{j}} r}$.

Voltamo-nos agora para alguns elementos de álgebra linear que serão importantes para o que será feito adiante. Em linhas gerais, nossa intenção é mostrar que se $E(G)$ for uma Base de Gauss para $I$, então $G$ será uma Base de Gröbner. A partir disso, encontraremos as condições necessárias para que isso ocorra e um refinamento dessas condições nos dará o teste procurado para decidir se $G$ é uma Base de Gröbner para $I$.

Definição 4.2.1 (Conjunto Escalonado) Dizemos que um conjunto $V$ de $K \Gamma$ é escalonado se não existem dois elementos $v_{1}$ e $v_{2}$ em $V$ tais que $\widehat{v_{1}}=\widehat{v_{2}}$.

Lema 4.2.18 Todo conjunto escalonado é linearmente independente.

Demonstração. Seja $V$ um subconjunto escalonado da álgebra $K \Gamma$. Assuma que temos uma combinação linear de elementos de $V$ igual a zero em que os coeficientes são todos não nulos:

$$
0=\sum_{i=1}^{m} \alpha_{i} v_{i}
$$

com cada $0 \neq \alpha_{i}$ um escalar e $v_{i}$ em $V$. Como $V$ é escalonado, os termos líderes dos elementos $v_{i}$ são dois a dois distintos. Nas, como o conjunto $\left\{\widehat{v}_{i} \mid 1 \leq i \leq m\right\}$ é finito, podemos escolher seu elemento máximo; seja $\widehat{v_{j}}$ esse elemento. Mas então $\widehat{v_{j}}$ é maior que todos os outros monômios que aparecem na soma acima. Com isso, ele não pode ser cancelado na combinação linear acima, pois ele não pode aparecer no suporte de nenhum $v_{i}$, para $i \neq j$ e não pode ser combinação linear de outros monômios (uma vez que $\mathcal{P}$ é uma base), o que nos leva a concluir que seu coeficiente $\left(\alpha_{j}\right)$ é zero, uma contradição. 
Utilizando a definição de $E(G)$ e o Lema 4.2.12, chegamos ao seguinte lema.

Lema 4.2.19 O conjunto $E(G)$ é escalonado.

Lema 4.2.20 Seja $h=\sum_{i=1}^{m} \alpha_{i} l_{i} g_{i} r_{i}$ uma combinação linear de elementos de $E(G)$. Então $\widehat{h} \in$ $\widehat{E(G)}$.

Demonstração. Se os elementos da combinação linear acima estão todos agrupados, tome $1 \leq j \leq m$ tal que $l_{j} \widehat{g_{j}} r_{j}$ é o maior elemento do conjunto formado pelos termos lideres dos elementos da combinação linear acima (ele existe pelo fato desse conjunto ser finito e totalmente ordenado). Logo, $l_{j} \widehat{g_{j}} r_{j}$ não aparece no suporte de nenhum $l_{i} g_{i} r_{i}$, para $i \neq j$, pela maximalidade de $l_{j} \widehat{g_{j}} r_{j}$ e pelo fato de $E(G)$ ser escalonado. Portanto, $l_{j} \widehat{g}_{j} r_{j}$ não é cancelado na soma acima, donde segue que ele é o termo líder de $h$.

Relembramos o importante conceito de Base de Gauss para o contexto de álgebras de caminhos e adaptamos a Definição 2.2.2 para este contexto.

Definição 4.2.2 (Base de Gauss) Seja $R$ uma álgebra com base multiplicativa $\mathcal{B}$ sobre a qual está fixada uma ordem admissivel $\leq$ e seja $I$ um ideal de $R$. Então uma base $B$ de $I$ escalonada é dita uma Base de Gauss de I (com respeito $a \leq$ ) se $\widehat{B}=\widehat{I}$.

Note que a partir da definição é imediato ver que uma Base de Gauss de um ideal $I$ é também uma Base de Gröbner para $I$.

Proposição 4.2.21 $O$ conjunto $G$ é uma Base de Gröbner para $I$ se e somente se $E(G)$ for uma Base de Gauss para I.

Demonstração. Pelos Lemas 4.2.19 e 4.2.18, temos que $E(G)$ é um conjunto escalonado e, portanto, linearmente independente.

Assuma que $G$ é uma Base de Gröbner para $I$. Mas então, pelo Lema 4.2.16, temos que $\widehat{E(G)}=\widehat{\mathcal{G}}=\widehat{I}$. Resta então mostrar que $E(G)$ gera $I$ como espaço vetorial. Suponha que não e vamos mostrar que isso nos leva a uma contradição.

Tome $f$ em $I$ um contra exemplo de termo líder mínimo. Mas, como $f$ pertence a $I$ e $G$ é uma Base de Gröbner, segue que $\widehat{f}$ pertence a $\widehat{\mathcal{G}}=\widehat{E(G)}$. Logo, existe $v$ em $E(G)$ tal que $\widehat{v}=\widehat{f}$. Se tomamos $f^{\prime}=f-\alpha v, \operatorname{com} \alpha$ um coeficiente tal que os termos líderes de $f$ e $v$ se cancelam na diferença anterior, teremos um elemento de termo líder menor que o termo líder de $f$. Logo, pela minimalidade de $f$, segue que $f^{\prime}=0$, o que encerra a demonstração ou $f^{\prime}=\sum_{i=1}^{m} \alpha_{i} v_{i}$, com $\alpha_{i}$ escalares e $v_{i}$ em $E(G)$, para todo $1 \leq i \leq m$. Assim, temos que $f=\sum_{i=1}^{m} \alpha_{i} v_{i}+\alpha v$, uma combinação linear de elementos de $E(G)$, nos levando a uma contradição com a hipótese de existência de $f$.

Para ver a implicação reversa, assuma que $E(G)$ é Base de Gauss para $I$ e mostraremos que $G$ é uma Base de Gröbner. Tome então $f$ em $I$ um elemento qualquer. Como $E(G)$ é Base de Gauss, existe $v=l_{i} r$ em $E(G)$, tal que $\widehat{v}=\widehat{f}$, donde segue que $g_{i} \in G$ divide $\widehat{f}$ e o resultado está demonstrado. 
Em seguida exploramos as condições que são necessárias para que $E(G)$ seja uma Base de Gauss de $I$. Iniciamos com o importante conceito de representação forte de Gauss.

Definição 4.2.3 (Representação forte de Gauss) Dizemos que um elemento $\lg _{j} r$ de $\mathcal{G}$ possui uma representação forte de Gauss (em termos de G) se

$$
l g_{j} r=\alpha \lambda g_{i} \rho+\sum_{k, u} \alpha_{k, u} l_{k, u} g_{k} r_{k, u} \text { (cada somando é não nulo) }
$$

satisfazendo $l \widehat{g_{j}} r=\lambda \widehat{g_{i}} \rho$ e $l_{k, u} \widehat{g_{k}} r_{k, u}<l \widehat{g_{j}} r$. Os elementos da forma $l_{k, u} g_{k} r_{k, u}$ e $\lambda g_{i} \rho$ pertencem a $E(G), \alpha_{k, u}$ são escalares, para todos $u, k$ e $\alpha=\operatorname{cl}\left(l g_{j} r\right) / c l\left(\lambda g_{i} \rho\right)$.

Proposição 4.2.22 O conjunto $E(G)$ é Base de Gauss para I se e somente se todo elemento de $\mathcal{G}$ tem uma representação forte de Gauss em termos de $G$.

Demonstração. Para ver que o fato de $E(G)$ ser Base de Gauss implica que todo elemento de $\mathcal{G}$ possui uma representação forte de Gauss, tome um contra exemplo $l g_{i} r$ em $\mathcal{G}$ de termo líder mínimo. Mas, como $E(G)$ é Base de Gauss, segue que existe um elemento $l^{\prime} g_{j} r^{\prime}$ de $E(G)$ tal que $l^{\prime} \widehat{g_{j}} r^{\prime}=l \widehat{g_{i}} r$, donde $l g_{i} r-\alpha l^{\prime} g_{j} r^{\prime}$ é zero ou possui uma representação forte de Gauss, pela minimalidade de $l \widehat{g}_{i} r$ e segue o resultado.

Para verificar a implicação reversa, assuma que todo elemento de $\mathcal{G}$ possui uma representação forte de Gauss e vejamos que $E(G)$ é Base de Gauss. Pelos Lemas 4.2 .19 e 4.2.18, temos que $E(G)$ é um conjunto escalonado e, portanto, linearmente independente, de forma que basta verificarmos que $E(G)$ é conjunto gerador e que $\widehat{E(G)}=\widehat{I}$.

Mas a primeira parte segue imediatamente do fato de que $\mathcal{G}$ é um conjunto gerador para $I$ e da hipótese da proposição. A segunda parte segue da hipótese de que todo elemento de $\mathcal{G}$ possui uma representação forte de Gauss, do fato de $\mathcal{G}$ ser um conjunto gerador para $I$ (como espaço vetorial) e do Lema 4.2.20.

Enfraquecendo a exigência de que os elementos de uma representação forte de Gauss estejam em $E(G)$, chegamos à noção de representação fraca de Gauss.

Definição 4.2.4 (Representação fraca de Gauss) Dizemos que um elemento $\lg _{j} r \in \mathcal{G}$ possui uma representação fraca de Gauss (em termos de G) se

$$
l g_{j} r=\alpha \lambda g_{i} \rho+\sum_{k, u} \alpha_{k, u} l_{k, u} g_{k} r_{k, u} \text { (cada somando é não nulo) }
$$

satisfazendo $\lg _{j} r=\lambda \widehat{g}_{i} \rho,\left(\lambda, \rho, g_{i}\right)<_{T}\left(l, r, g_{j}\right)$ e $l_{k, u} \widehat{g_{k}} r_{k, u}<l \widehat{g_{j}} r$. Os elementos da forma $l_{k, u} e$ $r_{k, u}$ pertencem a $\mathcal{P}, \alpha_{k, u}$ são escalares, para todos $u, k$ e $\alpha=\operatorname{cl}\left(l_{g_{j}} r\right) / \operatorname{cl}\left(\lambda g_{i} \rho\right)$.

Note que a definição implica que cada $\left(l_{k \cdot u}, r_{k, u}, g_{k}\right)<_{T}\left(l, r, g_{j}\right)$. Ainda, um elemento de $\mathcal{G} \backslash E(G)$ que possui uma representação forte de Gauss também possui uma representação fraca de Gauss, como nos mostra o seguinte lema.

Lema 4.2.23 Seja $\lg _{j} r$ em $\mathcal{G} \backslash E(G)$, tal que $\lg _{j} r$ possui uma representação forte de Gauss. Então $l_{g_{j}} r$ possui uma representação fraca de Gauss. 
Demonstração. Tome uma representação forte de Gauss da forma:

$$
l g_{j} r=\lambda g_{i} \rho+\sum_{k, u} \alpha_{k, u} l_{k, u} g_{k} r_{k, u}
$$

Observando as definições de representação fraca e forte de Gauss, vemos que basta mostrar que $\left(\lambda, \rho, g_{i}\right)<_{T}\left(l, r, g_{j}\right)$; mas isso segue imediatamente dos fatos de $l \widehat{g}_{j} r$ ser igual a $\lambda \widehat{g}_{i} \rho,\left(\lambda, \rho, g_{i}\right) \neq\left(l, r, g_{j}\right)$ (pois $(l, r) \notin E(j)$ por hipótese) e do Lema 4.2.17.

Proposição 4.2.24 Cada elemento de $\mathcal{G}$ possui uma representação forte de Gauss em termos de $G$ se e somente se, para cada $j$, para cada par $(l, r)$ em $T(j) \backslash N u l l(j)$, o elemento $l_{j} r$ tem uma representação fraca de Gauss em termos de $G$.

Demonstração. Para ver que o fato de cada elemento de $\mathcal{G}$ possui uma representação forte de Gauss implica que para cada $j$, para cada par $(l, r)$ em $T(j) \backslash N u l l(j)$, o elemento $l g_{j} r$ tem uma representação fraca de Gauss em termos de $G$, tome um $j$ qualquer e $(l, r)$ em $T(j) \backslash N u l l(j)$. Pelas definições de $T(j)$ e $E(G)$, vemos que $l g_{j} r$ não pertence a $E(G)$, e o resultado segue pelo Lema 4.2.23.

Vejamos agora a implicação reversa. Claramente todo elemento de $E(G)$ possui uma representação forte de Gauss trivialmente. Definimos então o conjunto:

$H:=\left\{\left(\lambda, \rho, g_{i}\right) \in T \mid \lambda g_{i} \rho \in \mathcal{G} \backslash E(G)\right.$ e não possui uma representação forte de Gauss $\}$

Se $H$ é vazio, então não há o que demonstrar. Se $H$ é não vazio, tome um elemento $\left(l, r, g_{j}\right)$ mínimo em $H$ (segundo $<_{T}$ ). Mas então, por hipótese, $l g_{j} r$ possui uma representação fraca de Gauss:

$$
l g_{j} r=\lambda g_{i} \rho+\sum_{k, u} \alpha_{k, u} l_{k, u} g_{k} r_{k, u}
$$

Ainda, pela minimalidade de $\left(l, r, g_{j}\right), \lambda g_{i} \rho$, bem como cada $l_{k, u} g_{k} r_{k, u}$ possuem representações fortes de Gauss, de onde segue que $l g_{j} r$ também possui uma representação forte de Gauss, contradizendo a suposição de $H$ ser não vazio.

\subsubsection{S-elementos e Sobreposições}

Vamos à definição de elementos que serão fundamentais para o que segue.

Definição 4.2 .5 (S-elemento) Sejam $\left(l, r, g_{j}\right)$ e $\left(l^{\prime}, r^{\prime}, g_{i}\right)$ em $T$ tais que $l \widehat{g_{j}} r=l^{\prime} \widehat{g_{i}} r^{\prime}$. Então um $S$-elemento envolvendo as triplas $\left(l, r, g_{j}\right)$ e $\left(l^{\prime}, r^{\prime}, g_{i}\right)$ é um elemento da forma:

$$
S\left(i, j, l, r, l^{\prime}, r^{\prime}\right):=l g_{i} r-\alpha l^{\prime} g_{j} r^{\prime}
$$

onde $\alpha=\operatorname{cl}\left(\lg _{i} r\right) / \operatorname{cl}\left(l^{\prime} g_{j} r^{\prime}\right)$. 
Note que o termo líder de um S-elemento $S\left(i, j, l, r, l^{\prime}, r^{\prime}\right)$ é sempre menor que $l \widehat{g_{i}} r=l^{\prime} \widehat{g_{j}} r^{\prime}$.

Diremos ainda que um S-elemento $S\left(i, j, l, r, l^{\prime}, r^{\prime}\right)$ tem uma representação de Gröbner (em termos de $G$ ) quando

$$
S\left(i, j, l, r, l^{\prime}, r^{\prime}\right)=\sum_{k, u} \alpha_{k, u} l_{k, u} g_{k} r_{k, u} \text { (cada somando é não nulo) }
$$

com $\alpha_{k, u}$ escalares e $l_{k, u} g_{k} r_{k, u}$ em $\mathcal{G}$, satisfazendo $l_{k, u} \widehat{g_{k}} r_{k, u}<l \widehat{g_{i}} r=l^{\prime} \widehat{g}_{j} r^{\prime}$

Deve estar claro para o leitor que, pela Proposição 4.1.2, todo S-elemento que se reduz para zero sobre $G$ também possui uma representação de Gröbner em termos de $G$. Isso ocorre pelo fato de o termo líder de um S-elemento $S\left(i, j, l, r, l^{\prime}, r^{\prime}\right.$ ) ser estritamente menor que $l \widehat{g}_{i} r=l^{\prime} \widehat{g}_{j} r^{\prime}$ (devido ao cancelamento desses termos no S-elemento). Resumimos esta observação na seguinte afirmação para futura referência.

Afirmação 4.2.25 Seja $S\left(i, j, l, r, l^{\prime}, r^{\prime}\right)$ um $S$-elemento que se reduz para zero sobre $G$. Então $S\left(i, j, l, r, l^{\prime}, r^{\prime}\right)$ possui uma representação de Gröbner em termos de $G$.

O seguinte resultado segue imediatamente das definições de S-elemento e de representação fraca de Gauss.

Lema 4.2.26 Seja $(l, r)$ em $T(i) \backslash N u l l(i)$, para algum $i \in \mathcal{I}$. Então $l_{i} r$ possui uma representação fraca de Gauss se e somente se existe um S-elemento $S\left(i, j, l, r, l^{\prime}, r^{\prime}\right)$, com $\left(l^{\prime}, r^{\prime}, g_{j}\right)<_{T}\left(l, r, g_{i}\right)$, que possui uma representação de Gröbner em termos de $G$.

Com o que temos até aqui, se, para cada $i$ em $\mathcal{I}$, para cada elemento $(l, r)$ de $T(i) \backslash N u l l(i)$, encontrarmos um S-elemento do qual faz parte outra tripla menor (de acordo com $<_{T}$ ) que $\left(l, r, g_{i}\right)$ e mostrarmos que ele possui uma representação de Gröbner em termos de $G$, então $G$ é uma Base de Gröbner.

O Lema 4.2.14 nos dá a boa notícia de que, para todos $(l, r)$ em $T(i) \backslash N u l l(i)$ (para todo $i$ em $\mathcal{I}$ ) sempre podemos encontrar elementos menores que $\left(l, r, g_{i}\right)$ e construir S-elementos com esses elementos menores, restando apenas verificar se esse S-elemento possui uma representação de Gröbner. A má notícia fica por conta do Exemplo 4.2.3, que nos mostra que $T(i)$ pode ser infinitamente gerado.

Precisamos então encontrar um conjunto finito de S-elementos (ao menos no caso em que $G$ for finito) de forma que, se cada elemento desse conjunto possui uma representação de Gröbner em termos de $G$, então cada S-elemento também possui uma tal representação. Com esse propósito, introduzimos os conceitos de relação de sobreposição e de divisão.

Definição 4.2.6 (Relação de Sobreposição) Dados dois elementos $g_{i}$ e $g_{j}$ de $G$, uma sobreposição envolvendo $g_{i}$ e $g_{j}$ é uma quádrupla $(i, j, l, r)$ satisfazendo:

- l e r são caminhos de comprimento maior que zero.

- $l \widehat{g_{i}}=\widehat{g}_{j} r \neq 0$.

- O termo líder de $g_{i}$ não divide $r$ e o termo líder de $g_{j}$ não divide $l$. 
Dada uma sobreposição $(i, j, l, r)$, diremos que uma relação de sobreposição envolvendo $g_{i}$ e $g_{j}$ é dada por

$$
O(i, j, l, r)=l g_{i}-\alpha g_{j} r
$$

$\operatorname{com} \alpha=\operatorname{cl}\left(l_{i}\right) / \operatorname{cl}\left(g_{j} r\right)$.

Definição 4.2.7 (Relação de Divisão) Dados dois elementos distintos $g_{i}$ e $g_{j}$ de $G$, dizemos que existe uma relação de divisão envolvendo $g_{i}$ e $g_{j}$ se $u \widehat{g_{j}} v=\widehat{g}_{i}$, com $u$ e $v$ caminhos. Neste caso, essa relação de divisão é dada por:

$$
D(i, j, u, v)=g_{i}-\alpha u g_{j} v
$$

$\operatorname{com} \alpha=\operatorname{cl}\left(g_{i}\right) / \operatorname{cl}\left(u g_{j} v\right)$.

Observamos ainda que, se $h$ e $w$ são dois elementos de um subconjunto qualquer de $K \Gamma$ (não necessariamente ordenado), denotaremos sobreposições, relações de sobreposição e relações de divisão envolvendo $h$ e $w$, respectivamente por $(h, w, l, r), O(h, w, l, r)$ e $D(h, w, u, v)$.

Note que tanto as relações de sobreposição quanto as de divisão constituem casos particulares de S-elementos.

Nos referiremos sempre de maneira vaga a relações de sobreposição (resp. divisão) envolvendo $g_{i}$ e $g_{j}$ para denotar todas as relações da forma $O(i, j, l, r)$ ou $O(j, i, l, r)$ (resp. $D(i, j, u, v)$ ou $D(j, i, u, v))$.

No que segue, mostraremos que as relações de sobreposição e de divisão constituem exatamente o conjunto finito de que precisávamos para nossos testes (no caso em que $G$ é finito). Em outras palavras, veremos que basta considerar as relações de sobreposição e de divisão envolvendo os elementos de $G$ para decidir se ele é uma Base de Gröbner.

Antes, vamos a um lema técnico.

Lema 4.2.27 Seja $S\left(i, j, l, r, l^{\prime}, r^{\prime}\right)$ um S-elemento tal que $\widehat{g}_{i}$ divide $l^{\prime}$ ou $\widehat{g_{j}}$ divide l. Então esse $S$-elemento possui uma representação de Gröbner em termos de $G$.

Demonstração. Mostraremos o caso em que $\widehat{g_{i}}$ divide $l^{\prime}$; o outro caso é completamente análogo. Mas então, teremos que $l^{\prime}=l_{1} \widehat{g_{i}} l_{2}$, o que implica $r=r_{1} \widehat{g_{j}} r_{2}$ e podemos concluir que $l_{1}=l, r_{2}=r^{\prime}$ e $r_{1}=l_{2}$. Essa situação pode ser vista no seguinte diagrama.

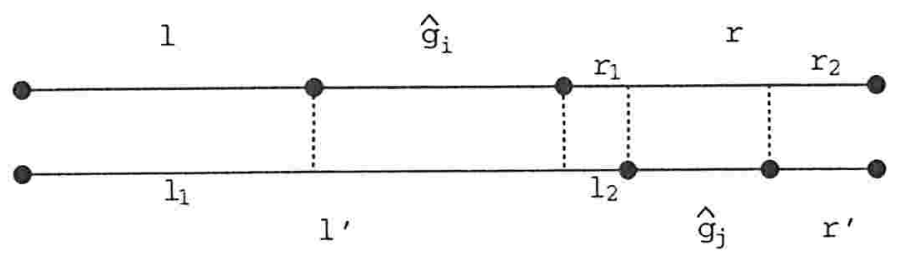

Denotamos:

$$
g_{i}=\operatorname{cl}\left(g_{i}\right) \widehat{g}_{i}+\sum_{k=1}^{m_{i}} \alpha_{k} a_{k}
$$


$\mathrm{e}$

$$
g_{j}=c l\left(g_{j}\right) \widehat{g_{j}}+\sum_{h=1}^{m_{j}} \beta_{h} b_{h}
$$

tais que $\alpha_{k}, \beta_{h}$ são escalares, $a_{k}$ é um caminho menor que $\widehat{g}_{j}$ e $b_{h}$ é um caminho menor que $\widehat{g}_{i}$, para todos $1 \leq k \leq m_{j}$ e $1 \leq h \leq m_{i}$.

Em vista das igualdades da hipótese e da notação acima, se denotamos por $\alpha=$ $c l\left(l_{i} r\right) / c l\left(l^{\prime} g_{j} r^{\prime}\right)$, podemos escrever:

$$
\begin{aligned}
S\left(i, j, l, r, l^{\prime}, r^{\prime}\right) & =l g_{i} r-\alpha l^{\prime} g_{j} r^{\prime}=l g_{i} r_{1} \widehat{g_{j}} r^{\prime}-\alpha l \widehat{g}_{i} r_{1} g_{j} r^{\prime} \\
& =l g_{i} r_{1}\left(\frac{1}{c l\left(g_{j}\right)} g_{j}-\sum_{h=1}^{m_{j}} \beta_{h} b_{h}\right) r^{\prime}-\alpha l\left(\frac{1}{c l\left(g_{i}\right)} g_{i}-\sum_{k=1}^{m_{i}} \alpha_{k} a_{k}\right) r_{1} g_{j} r^{\prime} \\
& =l\left(\sum_{k=1}^{m_{i}} \alpha_{k} a_{k}\right) r_{1} \frac{1}{c l\left(g_{j}\right)} g_{j} r^{\prime}-\alpha l \frac{1}{c l\left(g_{i}\right)} g_{i} r_{1}\left(\sum_{h=1}^{m_{j}} \beta_{h} b_{h}\right) r^{\prime}
\end{aligned}
$$

Note que as multiplicações são todas não nulas pela uniformidade dos elementos de $G$ e pela definição de S-elemento (exigindo que todas as triplas estejam em $T$ ). Com isso, como $a_{k}<\widehat{g}_{j}$ e $b_{h}<\widehat{g}_{i}$, para todos $1 \leq k \leq m_{j}$ e $1 \leq h \leq m_{i}$, teremos que os elementos $l a_{k} r_{1} \widehat{g_{i}} r^{\prime}$ e $l \widehat{g_{j}} r_{1} b_{h} r^{\prime}$ serão menores que $l \widehat{g}_{j} r$, para todos $1 \leq k \leq m_{j}$ e $1 \leq h \leq m_{i}$ e segue que $S\left(i, j, l, r, l^{\prime}, r^{\prime}\right)$ possui uma representação de Gröbner em termos de $G$.

Proposição 4.2.28 Seja $G$ um conjunto de elementos uniformes de forma que todas as relações de sobreposição e de divisão envolvendo elementos de $G$ possuem representações de Gröbner em termos de $G$. Então todo $S$-elemento da forma $S\left(i, j, l, r, l^{\prime}, r^{\prime}\right)$, com $g_{i}$ e $g_{j}$ em $G$ também possui uma representação de Gröbner em termos de $G$.

Demonstração. Seja $G$ nas hipóteses do enunciado e tome um S-elemento da forma $S\left(i, j, l, r, l^{\prime}, r^{\prime}\right)$. Claro que pela definição de S-elemento, temos que $l \widehat{g}_{j} r=l^{\prime} \widehat{g}_{i} r^{\prime}$. Ainda, pelo Lema 4.2.27, podemos assumir que $\widehat{g_{i}}$ não divide $l$ e $\widehat{g_{j}}$ não divide $l^{\prime}$. Portanto temos três casos:

- O comprimento de $l$ é menor que o de $l^{\prime}$.

Para esta situação, temos dois subcasos:

- $\widehat{g}_{j}$ não divide $\widehat{g_{i}}$.

Neste caso, existem divisores próprios $v$ e $w$ de $\widehat{g_{j}}$ e $\widehat{g_{i}}$, respectivamente. Ainda, esses divisores são tais que $l w=l^{\prime}, v r^{\prime}=r, w$ não é divisível por $\widehat{g_{i}}$ e $v$ não é divisível por $\widehat{g_{j}}$. A situação é ilustrada pelo diagrama a seguir.

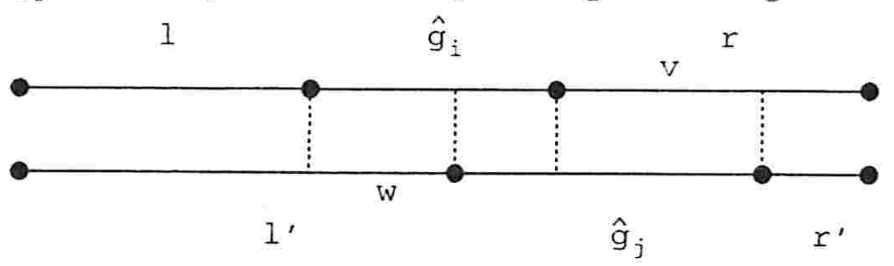


Mas então,

$$
\begin{aligned}
S\left(i, j, l, r, l^{\prime}, r^{\prime}\right) & =l g_{i} r-l^{\prime} g_{j} r^{\prime} \\
& =l g_{i} v r^{\prime}-l w g_{j} r^{\prime} \\
& =-l O(j, i, w, v) r^{\prime}
\end{aligned}
$$

Mas, por hipótese, $O(j, i, w, v)$ possui uma representação de Gröbner. Logo concluímos:

$$
S\left(i, j, l, r, l^{\prime}, r^{\prime}\right)=-l\left(\sum_{k, u} \alpha_{k, u} l_{k, u} g_{k} r_{k, u}\right) r^{\prime}=\sum_{k, u} \beta_{k, u} l l_{k, u} g_{k} r_{k, u} r^{\prime}
$$

onde $\beta_{k, u}=-\alpha_{k, u}$, para todos $k, u$. Note que, como os elementos $l_{k, u} g_{k} r_{k, u}$ são uniformes, cada $l l_{k, u} g_{k} r_{k, u} r^{\prime}$ ou é zero ou nenhuma das multiplicações é zero. Ainda, o fato de $l_{k, u} g_{k} r_{k, u}$ ser menor que $w \widehat{g_{j}}$ (pela definição de representação de Gröbner) implica $l l_{k, u} \widehat{g_{k}} r_{k, u} r^{\prime}<l w \widehat{g_{j}} r^{\prime}=l^{\prime} \widehat{g_{j}} r^{\prime}=l \widehat{g}_{i} r\left(\right.$ se $\left.l l_{k, u} g_{k} r_{k, u} r^{\prime} \neq 0\right)$, para todos $k, u$.

Podemos então remover os somandos da soma acima que são iguais a zero e teremos uma representação de Gröbner para $S\left(i, j, l, r, l^{\prime}, r^{\prime}\right)$, encerrando este caso.

- $\widehat{g_{j}}$ divide $\widehat{g}_{i}$.

Procedemos de forma análoga ao que foi feito para o caso anterior, mas utilizando a relação de divisão $D(i, j, w, v)$ (que, por hipótese, possui uma representação de Gröbner). A situação pode ser vista esquematicamente no diagrama a seguir:

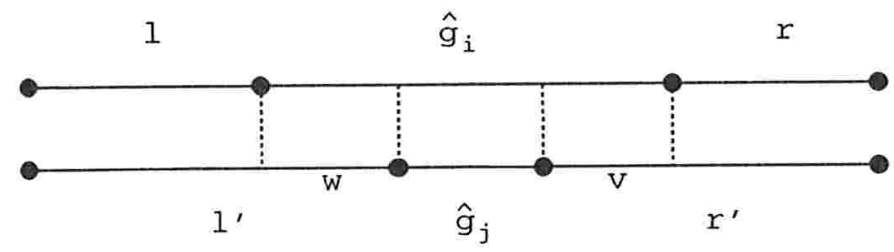

- O comprimento de $l$ é maior que o de $l^{\prime}$.

A demonstração para este caso é análoga à do caso anterior, fazendo uso de uma relação da forma $O(i, j, w, v)$ para o caso em que $\widehat{g_{i}}$ não divide $\widehat{g_{j}}$ e $D(j, i, w, v)$ para o caso em que $\widehat{g_{i}}$ divide $\widehat{g_{j}}$.

- O comprimento de $l$ é igual ao de $l^{\prime}$.

Aqui temos três possibilidades:

- O comprimento de $\widehat{g}_{i}$ é menor que o comprimento de $\widehat{g_{j}}$.

Teste caso, procedemos de forma análoga ao que tem sido feito, utilizando uma relação de divisão da forma $D\left(j, i, \mathrm{o}\left(\widehat{g}_{i}\right), w\right)$.

- O comprimento de $\widehat{g_{i}}$ é maior que o comprimento de $\widehat{g_{j}}$.

Esta situação é análoga à anterior, se utilizarmos uma relação de divisão da forma $D\left(i, j, \mathrm{o}\left(\widehat{g_{j}}\right), w\right)$. 
- O comprimento de $\widehat{g}_{i}$ é igual ao comprimento de $\widehat{g_{j}}$.

Neste caso, o S-elemento é zero e não há o que mostrar.

Com o que temos até aqui, reduzimos nosso problema de verificar se $G$ é uma Base de Gröbner para testar, para cada par de elementos $g_{i}$ e $g_{j}$ de $G$, se cada relação de sobreposição e de divisão envolvendo $g_{i}$ e $g_{j}$ possui uma representação de Gröbner em termos de $G$. O próximo resultado nos mostra que isso nos deixa com um número finito de verificações, se $G$ for finito.

Proposição 4.2.29 Dados dois elementos $g_{i}$ e $g_{j}$ de $G$, existe apenas um número finito de relações de sobreposição e de divisão envolvendo $g_{i}$ e $g_{j}$.

Demonstração. Considere uma sobreposição $(i, j, l, r)$. Note que, pela definição de sobreposição, o comprimento de $l$ é menor ou igual ao comprimento de $\widehat{g_{j}}$ e o comprimento de $r$ é menor ou igual ao comprimento de $\widehat{g}_{i}$. O resultado segue então do fato de que assumimos que a álgebra $K \Gamma$ é finitamente gerada (e, portanto, há apenas um número finito de caminhos limitados por um comprimento).

Para ver que o número de relações de divisão entre $g_{i}$ e $g_{j}$ é finito, basta notar que em uma álgebra de caminhos, existe apenas um número finito de formas de um monômio dividir outro.

\subsubsection{Conclusões}

Podemos resumir o que foi visto até aqui na seguinte proposição.

Proposição 4.2.30 Seja KГ uma álgebra de caminhos finitamente gerada. Considere a base multiplicativa $\mathcal{P}$ de $K \Gamma$ formada pelos caminhos em $\Gamma$ e assuma que temos uma ordem admissivel fixada em $\mathcal{P}$.

Seja $G$ um subconjunto de $K \Gamma$ (possivelmente infinito) composto somente por elementos uniformes. Se cada relação de sobreposição e cada relação de divisão envolvendo elementos de $G$ possui uma representação de Gröbner em termos de $G$, então $G$ é uma Base de Gröbner.

No caso em que $G$ é finito, podemos utilizar a Afirmação 4.2 .25 para tornar o teste descrito na proposição acima algorítmico, por meio do Algoritmo da Divisão. Com isso, podemos calcular as relações de sobreposição e de divisão envolvendo elementos de $G$ e verificar se a saída do Algoritmo da Divisão para cada uma delas é zero.

Por um lado, se $G$ for uma Base de Gröbner, a saída para cada uma delas será zero necessariamente, pela Proposição 4.1.4 e pelo Corolário 3.1.7. Por outro lado, se todas as saídas são zero, segue que cada uma das relações de sobreposição e de divisão envolvendo elementos de $G$ se reduz para zero sobre $G$ e, portanto, possui uma representação de Gröbner em termos de $G$. Neste caso, $G$ é uma Base de Gröbner, pela Proposição 4.2.30.

Contudo, pelo que foi desenvolvido até aqui, vemos que a hipótese de finitude de $G$ imposta acima é necessária somente por duas razões: garantir que o número de relações seja finito e garantir que a execução do Algoritmo da Divisão termine. Logo, se tivermos em mãos um conjunto $G$ infinito, mas tivermos a garantia de que, para cada relação de sobreposição ou de divisão envolvendo elementos de 
$G$, existe uma seqüência (finita) de escolhas de divisores em $G$ que faz com que a saída do Algoritmo da Divisão seja zero, $G$ será uma Base de Gröbner.

Resumimos os comentários e resultados apresentados até aqui no Teorema 4.2.31.

Teorema 4.2.31 Seja $R=K \Gamma$ uma álgebra de caminhos finitamente gerada. Considere a base multiplicativa $\mathcal{P}$ de $R$ formada pelos caminhos em $\Gamma$ e assuma que temos uma ordem admissivel fixada em $\mathcal{P}$. Seja $G$ um subconjunto de $R$ (possivelmente infinito) composto somente por elementos uniformes. Então as seguintes condições são equivalentes:

- O conjunto $G$ é uma Base de Gröbner.

- Cada relação de sobreposição e de divisão envolvendo elementos de $G$ se reduz para zero sobre algum subconjunto finito de $G$.

Utilizando a Proposição 4.2.29, chegamos ao seguinte corolário.

Corolário 4.2.32 Existe um algoritmo que recebe como entrada um subconjunto $G$ finito e uniforme de $K \Gamma$ e decide se $G$ é uma Base de Gröbner.

Como exemplo de aplicação dos resultados aqui apresentados, retomamos o Exemplo 3.2 .2 a fim de verificar que os conjuntos apresentados naquele exemplo são de fato Bases de Gröbner para o ideal $I$ nas ordens consideradas.

Exemplo 4.2.6 Considere o contexto do Exemplo 3.2.2. Assuma primeiramente que $a>b$ e vejamos que $G:=\left\{a b^{2 i-1} a-b^{2 i} a \mid i \geq 1\right\}$ constitui uma Base de Gröbner reduzida para $I$.

Para tanto, denote por $f_{i}$ o seguinte elemento:

$$
f_{i}:=a b^{2 i-1} a-b^{2 i} a
$$

e note que $G=\left\{f_{i} \mid i \geq 1\right\}$.

Vejamos primeiramente que $I=\langle G\rangle$. Claro que $f_{1}=f$, de modo que $I \subseteq\langle G\rangle$. Portanto, basta mostrar que $f_{i} \in I$, para todo $i \geq 1$. A prova desse fato segue por indução em $i$.

Se $i=1, f_{1}=f \in I$ e não há mais o que mostrar. Assuma portanto, que a afirmação de que todo $f_{j}$ pertence a $I$ para todo $1<j<i$ é verdadeira e mostrarmos que ela vale para $i$. Mas então

$$
f_{i}=f_{i-1} b a-a b^{2 i-3} f_{1}+b^{2 i-2} f_{1} \in I
$$

o que mostra que $I=\langle G\rangle$.

Vejamos agora que $G$ é uma Base de Gröbner. Para tanto, faremos uso do Teorema 4.2.31. Primeiro, veja que não existem relações de divisão entre elementos de $G$. Em seguida, note que, dados dois elementos $f_{i}$ e $f_{j}$, existem apenas duas sobreposições possiveis entre eles. 
Logo, tomamos $f_{h}$ e $f_{k}$ elementos quaisquer de $G$ e mostramos que a relação de sobreposição $O\left(f_{h}, f_{k}, a b^{2 k-1}, b^{2 h-1} a\right)$ se reduz para zero sobre $G$; o fato de $G$ ser uma Base de Gröbner seguirá então do Teorema 4.2.31. Assim:

$$
\begin{aligned}
O\left(f_{h}, f_{k}, a b^{2 k-1}, b^{2 h-1} a\right) & \\
& =a b^{2 k-1} f_{h}-f_{k} b^{2 h-1} a \\
& =-a b^{2 k-1} b^{2 h} a+b^{2 k} a b^{2 h-1} a \\
\Rightarrow_{f_{h}} & -a b^{2(k+h)-1} a+b^{2 k} a b^{2 h-1} a-\underbrace{b^{2 k} a b^{2 h-1} a+b^{2 k} b^{2 h} a}_{b^{2 k} f_{h}} \\
& =-a b^{2(k+h)-1} a+b^{2(k+h)} a=-f_{k+h}
\end{aligned}
$$

Com isso, todas as relações de sobreposição envolvendo elementos de $G$ se reduzem para zero sobre $G$, donde $G$ é uma Base de Gröbner.

Mas então, como $G$ é Base de Gröbner para $\langle G\rangle=I$, temos que $\widehat{I}=\langle\widehat{G}\rangle$. É evidente que não existem dois elementos em $\widehat{G}$ tais que um divide o outro. Logo, pelo Corolário $3.2 .10, \widehat{G}$ é o conjunto gerador monomial minimal de $\widehat{I}$ e concluímos que $G$ é uma Base de Gröbner minimal para $I$.

Além disso, $b^{2 i} a$ não é divisível por nenhum elemento de $\widehat{G}$ e temos que cada elemento de $G$ é reduzido. Logo, $G$ é a Base de Gröbner reduzida para $I$.

Claramente $G$ é um conjunto infinito. Portanto, $I$ não possui nenhuma Base de Gröbner finita na ordem dada, pelo Corolário 3.2.15.

Por outro lado, se tomamos deglex $\operatorname{com} b>a$, então $\widehat{f}=b^{2} a$. Como não há nenhuma sobreposição envolvendo $b^{2} a$, o Teorema 4.2.31 nos diz que $\{f\}$ é uma Base de Gröbner para $I$.

\subsubsection{Um Contra-exemplo}

Com o Teorema 4.2.31 demonstrado para as álgebras de caminhos seria natural perguntar se essa mesma propriedade é válida para casos mais gerais. O exemplo a seguir mostra que o Teorema 4.2.31 não pode ser estendido sem modificações para álgebras quaisquer. Desconhecemos generalizações desse teorema para contextos mais amplos que as álgebras de caminhos, o que contribui para nosso interesse por essas álgebras ao longo de todo o trabalho.

O questionamento inicial de que o teorema deveria ser falso para álgebras em geral foi proposto por nós. A partir dele, E. L. Green exibiu um exemplo que foi posteriormente refinado por nós dando origem ao que é apresentado em seguida.

Considere o grafo $\Gamma$ dado na figura abaixo. 


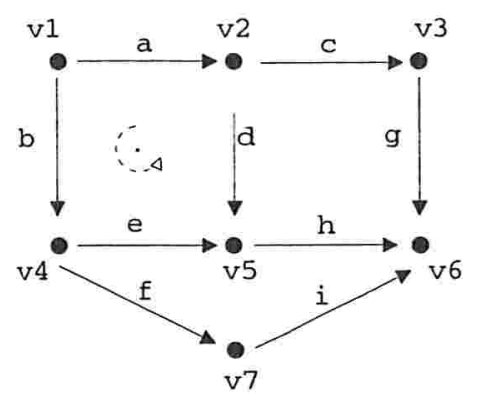

Seja $R=K \Gamma /\langle a d-b e\rangle$ e denotamos por $\pi$ a projeção canônica de $K \Gamma$ em $R$. Fixamos ainda a base multiplicativa $\mathcal{B}:=\pi(\mathcal{P}) \backslash\{0\}$ de $R$ (ela é multiplicativa pelo fato de $\langle a d-b e\rangle$ ser 2-nomial e pela Proposição 3.4.22) e a seguinte ordem admissível em $\mathcal{B}$ (tomamos uma ordem qualquer nos vértices e assumimos que eles são menores que os arcos): ${ }^{12}$

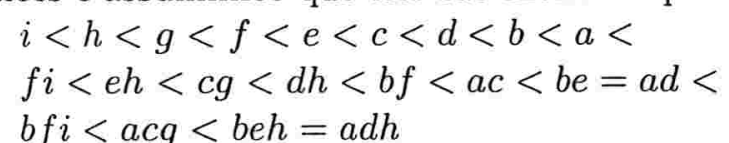

Não é difícil ver que os monômios listados acima são todos os elementos de $\mathcal{B}$. Ademais, não é difícil ver que os únicos elementos de $\mathcal{P}$ com mesma imagem por $\pi$ são os acima indicados (a verificação desse fato pode ser feita por meio da própria Teoria de Bases de Gröbner, se fixarmos uma ordem admissível em $\mathcal{P}$ e notarmos que $\{a d-b e\}$ é uma Base de Gröbner).

A verificação de que a ordem dada é de fato admissível pode ser feita analisando-se todas as possibilidades, uma vez que o conjunto dos caminhos é finito.

Considere agora $G$ o subconjunto de $R$ dado por $G=\{d h-c g, e h-f i\}$. Naturalmente, o conjunto dos termos líderes de $G, \widehat{G}$, é igual a $\{d h, e h\}$. Além disso, $a(d h-c g)-b(e h-f i)$ é um elemento de $\langle G\rangle$, mas

$$
\begin{aligned}
a(d h-c g)-b(e h-f i) & =(a d-b e) h-a c g+b f i \\
& =-a c g+b f i
\end{aligned}
$$

Portanto, o termo líder de $a(d h-c g)-b(e h-f i)$ é $a c g$ e $G$ não é uma Base de Gröbner. Por outro lado, vemos que $G$ está nas condições do Teorema 4.2 .31 (exceto pelo fato de $R$ não ser uma álgebra de caminhos), uma vez que ele é um conjunto uniforme e não existem relações de sobreposição nem de divisão envolvendo os elementos de $G$.

\subsubsection{Variações sobre o Lema do Diamante}

Descrevemos aqui como o Lema do Diamante pode ser utilizado para demonstrar o Teorema 4.2.31. Assumiremos como conhecida a terminologia de Bergman [Ber78], relembrando somente os principais conceitos e apresentamos o enunciado do Lema para conveniência do leitor.

Seja $X$ um alfabeto finito e considere a álgebra livre $K\langle X\rangle$. Fixamos um conjunto $S$ não vazio de pares da forma $\sigma=\left(W_{\sigma} . f_{\sigma}\right)$, com $W_{\sigma}$ em $X^{*}$ e $f_{\sigma}$ em $K\langle X\rangle$, que será chamado de sistema de redução. Ainda, dado $\sigma$ em $S$ e monòmios $a, b$ em $X^{*}$, definimos o endomorfismo de espaços vetoriais $r_{a \sigma b}: K\langle X\rangle \longrightarrow K\langle X\rangle$ definido sobre os monômios da base $X^{*}$ por

\footnotetext{
${ }^{12}$ A fim de nāo tornar a linguagem muito pesada, cometemos um abuso de notação e denotamos a projeção de um caminho $p$ em $R$ simplesmente por $p$.
} 


$$
r_{a \sigma b}(v)=\left\{\begin{array}{l}
v \text { se } v \neq a W_{\sigma} b \\
a f_{\sigma} b \text { se } v=a W_{\sigma} b
\end{array}\right.
$$

Ao longo desta seção, essas funções serão chamadas de funções de redução. Uma composição de tais funções será chamada de seqüência de reduções. Dada uma sequiência de reduções $r=\left(r_{1} r_{2} \cdots r_{n}\right)$ e um elemento $f$ de $K\langle X\rangle$, denotaremos por $r(f)$ a imagem de $f$ pela seqüência $r$, ou seja $r(f)=r_{n} r_{n-1} \cdots r_{1}(f)$.

Dado um elemento $f$ de $K\langle X\rangle$, diremos que uma redução $r_{a \sigma b}$ atua trivialmente em $f$ se o coeficiente de $a W_{\sigma} b$ em $f$ for zero. Ainda, um elemento $f$ de $K\langle X\rangle$ é dito irredutível se toda função de redução atua trivialmente em $f$.

Se $r=\left(r_{1} r_{2} \cdots r_{n}\right)$ é uma sequiência de reduções e $f$ é um elemento de $K\langle X\rangle$ tal que $r_{n} \cdots r_{1}(f)$ é um elemento irredutível, diremos que a seqüência $r$ é final em $f$. Um elemento $f$ é dito de redução finita se, para toda seqüência de reduções $\left(r_{1}, r_{2}, \ldots\right)$, existe $i_{0} \in \mathbb{N}^{*}$ tal que $r_{i}$ atua trivialmente em $r_{i-1} \cdots r_{1}(f)$, para todo $i>i_{0}$.

Ainda, um elemento $f$ de redução finita é dito de redução única se a imagem de $f$ por toda seqüência de reduções final em $f$ for sempre a mesma. Neste caso, denotaremos a imagem de $f$ por uma seqüência de reduções final em $f$ por $r_{S}(f)$. Dado um elemento $f$ de $K\langle X\rangle$, diremos que uma seqüência de reduções $r=\left(r_{1}, r_{2}, \ldots, r_{n}\right)$ é efetiva em $f$ se $r_{1}$ atua não trivialmente em $f$ e $r_{i}$ atua não trivialmente em $r_{i-1} \cdots r_{1}(f)$, para todo $2 \leq i \leq n$. $^{13}$

Dada uma ordem parcial $\leq$ em $X^{*}$, diremos que $\leq$ é uma ordem parcial de semigrupo se ela for compatível com a multiplicação em $X^{*}$, isto é, sempre que dois monômios $b, b^{\prime}$ forem tais que $b<b^{\prime}$, então $a b c<a b^{\prime} c$, para todos $a, c$ em $X^{*}$. Ainda, dado um sistema de redução $S$, diremos que $\leq$ é compatível com $S$ se, para todo $\sigma$ em $S, f_{\sigma}$ for zero ou for uma combinação linear de monômios menores que $W_{\sigma}$.

Uma ambigüidade de sobreposição é uma 5-tupla $s=\left(\sigma_{1}, \sigma_{2}, a, b, c\right)$, com $\sigma_{1}$ e $\sigma_{2}$ em $S, a, b$ e $c$ em $X^{*}, b \neq 1$, tal que $W_{\sigma_{1}}=b c$ e $W_{\sigma_{2}}=a b$. Diremos que uma ambigüidade de sobreposição $s=\left(\sigma_{1}, \sigma_{2}, a, b, c\right)$ é resolúvel se existirem seqüências de reduções $r$ e $r^{\prime}$ tais que

$$
r\left(a f_{\sigma_{1}}\right)=r^{\prime}\left(f_{\sigma_{2}} c\right)
$$

Uma ambigüidade de inclusão é uma 5-tupla $i=\left(\sigma_{1}, \sigma_{2}, a, b, c\right)$, com $\sigma_{1}$ e $\sigma_{2}$ em $S, a, b$ e $c$ em $X^{*}, \sigma_{1} \neq \sigma_{2}$, tal que $W_{\sigma_{1}}=b$ e $W_{\sigma_{2}}=a b c$. Diremos que uma ambigüidade de inclusão é resolúvel se existirem seqüèncias de reduções $r$ e $r^{\prime}$ tais que

$$
r\left(a f_{\sigma_{1}} c\right)=r^{\prime}\left(f_{\sigma_{2}}\right)
$$

Com isso, podemos enunciar o Lema do Diamante de Bergman.

Teorema 4.2.33 (Lema do Diamante) Seja $S$ um sistema de redução para a álgebra livre, $K\langle X\rangle$, $e \leq$ uma ordem parcial de semigrupo em $X^{*}$ com a condiçăo da cadeia descendente, de modo que $\leq$ e $S$ sejam compativeis. Então as seguintes condições são equivalentes.

1. Todas as ambigüidades de $S$ são resolúveis.

\footnotetext{
${ }^{13}$ A noção de seqüència de redução efetiva nāo é encontrada no trabalho original de Bergman. Ela foi introduzida por nós a fim de simplificar alguns argumentos que relacionam a teoria desenvolvida no Lema do Diamante com a Teoria de Bases de Gröbner.
} 
2. Todos os elementos de $R$ são de redução única.

3. Um sistema de representantes em $K\langle X\rangle$ para os elementos da álgebra quociente $Q=K\langle X\rangle / I$, determinada pelos geradores $X$ e relações $W_{\sigma}=f_{\sigma}(\sigma$ em $S)$, é dado pelo $K$-subespaço vetorial de $K\langle X\rangle$ gerado pelos monômios que são irredutiveis por funções de redução dadas pelo sistema de redução $S$.

Assuma então a notação da seção anterior e as hipóteses do Teorema 4.2 .31 (com $R=K\langle X\rangle)$ e vejamos como podemos demonstrá-lo fazendo uso do resultado acima. Antes de mais nada é preciso encontrar um sistema de redução $S$ que sirva para nossos propósitos.

Tome então $G=\left(g_{1}, g_{2}, \ldots\right)$ como antes (com $\mathcal{I}=\left\{i \mid g_{i} \in G\right\}$ ) e podemos assumir, sem perda de generalidade, que $\operatorname{cl}\left(g_{i}\right)=1$, para todo $i$ em $\mathcal{I}$. Claro que todo elemento $g_{i}$ de $G$ se escreve como $g_{i}=\widehat{g}_{i}+\sum_{b_{j} \in \operatorname{supp}\left(g_{i}\right) \backslash\left\{\widehat{g}_{i}\right\}} \alpha_{j} b_{j}$. Então, para cada $g_{i}$ em $G$, definimos um elemento $\sigma_{i}$ de $X^{*} \times K\langle X\rangle$ da seguinte forma:

$$
\sigma_{i}=\left(\widehat{g}_{i},-\sum_{b_{j} \in \operatorname{supp}\left(g_{i}\right) \backslash\left\{\widehat{g}_{i}\right\}} \alpha_{j} b_{j}\right)
$$

Tomando $S$ como o conjunto formado pelos elementos $\sigma_{i}$, para todo $i \in \mathcal{I}$, teremos um sistema de redução para $K\langle X\rangle$.

Note ainda que a presença de uma ordem admissível em $X^{*}$ nos fornece uma ordem nas hipóteses do Lema do Diamante: ela é uma ordem parcial de semigrupo em $X^{*}$ que satisfaz a condição de cadeia descendente (Proposição 1.1.1) e é compatível com $S$.

Mostraremos em seguida que a hipótese de que cada relação de sobreposição e de divisão envolvendo elementos de $G$ se reduz para zero sobre $G$ implica a condição 1 do Lema do Diamante.

Pelas definições de ambigüidade de sobreposição, de sobreposição e pela construção de nosso sistema de redução $S$, é imediato verificar que a cada ambigüidade de sobreposição de $S, s=$ $\left(\sigma_{i_{1}} \sigma_{i_{2}}, a, b, c\right)$, corresponde uma relação sobreposição $O\left(i_{1}, i_{2}, a, c\right)$ (e conseqüentemente, uma sobreposição $\left.\left(i_{1}, i_{2}, a, c\right)\right)$ envolvendo $g_{i_{1}}$ e $g_{i_{2}}$. Diremos então que $O\left(i_{1}, i_{2}, a, c\right)$ é a relação de sobreposição associada à ambigüidade de sobreposição $s$.

Analogamente, dada uma ambigüidade de inclusão $i=\left(\sigma_{i_{1}}, \sigma_{i_{2}}, a, b, c\right)$, podemos encontrar a relação de divisão associada à ambigüidade de inclusão $i$, dada por $D\left(i_{1}, i_{2}, a, c\right)$.

Apresentamos dois resultados que são de verificação imediata a partir das definições dadas. A primeira segue imediatamente da construção de $S$, do fato de o coeficiente líder de cada $g_{i}$ ser 1 e da definição de função de redução.

Afirmação 4.2.34 Cada $g_{i}$ em $G$ é da forma $g_{i}=W_{\sigma_{i}}-f_{\sigma_{i}}$.

Seja $f$ em $K\langle X\rangle$ e $r=r_{a \sigma_{2} b}$ uma função de redução. Então $r(f)=f-\alpha a g_{i} b$, onde $\alpha$ é o coeficiente de $a W_{\sigma_{i}} b=a \widehat{g}_{i} b$ em $f$. Ademais, $r$ não atua trivialmente em $f$ se e somente se $\alpha$ for diferente de zero.

Inversamente, se $f^{\prime}$ é um elemento de $K\langle X\rangle$ da forma $f^{\prime}=f-\alpha a g_{i} b$, onde $\alpha$ é o coeficiente de $a V_{\sigma_{i}} b=a \widehat{g}_{i} b$ em $f$, então existe uma função de redução $r=r_{a \sigma_{\imath} b}$ tal que $r(f)=f^{\prime}$. Ademais $r$ não atua trivialmente em $f$ se e somente se $\alpha$ for diferente de zero.

O seguinte lema decorre imediatamente da afirmação acima e da descrição do Algoritmo da Divisão (Algoritmo 4.1.1). 
Lema 4.2.35 Seja $f$ um elemento de $K\langle X\rangle$. Se $f$ se reduz para $f^{\prime}$ sobre $G$, então existe uma seqüência de reduções final e efetiva tal que $r(f)=f^{\prime}$.

Esse último resultado nos mostra então que toda execução do Algoritmo da Divisão corresponde a uma seqüência de reduções. Em outras palavras, aplicar o Algoritmo da Divisão sobre um elemento $f$ de $K\langle X\rangle$ (fornecendo o conjunto $G$ como parâmetro) ${ }^{14}$ é o mesmo que computar a imagem de $f$ por uma seqüência de reduções.

O exemplo a seguir nos mostra que o inverso nem sempre é verdadeiro; isto é nem toda seqüência de reduções final e efetiva equivale a uma seqüência de escolhas de divisores para a execução do Algoritmo da Divisão.

Exemplo 4.2.7 Tome $R=K\langle a, b, c, d\rangle$, considere como base multiplicativa o conjunto de todas as palavras sobre $\{a, b, c, d\}$ e fixe a ordem de grau e lexicografia, com $a>b>$ $c>d$. Considere $G=\left(g_{1}, g_{2}, g_{3}, g_{4}, g_{5}\right)$, onde

$$
\begin{array}{lll}
g_{1}=a b-a c & g_{2}=a a-a c & g_{3}=b a \\
g_{4}=a c-d^{2} & g_{5}=c^{2} &
\end{array}
$$

Seja $f=a b c-a c^{2}$ e considere a seguinte seqüência de reduções: $r=\left(r_{1 \sigma_{4} c} r_{1 \sigma_{1} c} r_{a \sigma_{5} 1}\right)$. Então $r(f)=d^{2} c$.

Por outro lado, se calculamos DIVISĀO $(G, f)$, teremos, na primeira iteração do processo, uma única opção de divisor para $\widehat{f}=a b c$ : $g_{1}$. Além disso, há somente uma forma de dividir $a b c$ por $\widehat{g_{1}}$. Assim, o processo fará a seguinte atribuição:

$$
g \leftarrow f-1 f_{1} c=0
$$

E o processo termina retornando 0 .

O seguinte resultado mostra que tal equivalência entre seqüencias de reduções e execuções do Algoritmo da Divisão existe quando $G$ é uma Base de Gröbner.

Lema 4.2.36 Se $G$ é uma Base de Gröbner, $f$ um elemento qualquer de $K\langle X\rangle$ e $r$ uma seqüência de reduções final em $f$, então $r(f)=N(f)$. Em particular, segue que todo elemento $f$ de redução finita é de redução única e que $r_{S}(f)=N(f)$.

Demonstração. A partir da definição de seqüência final de reduções e do fato de $G$ ser uma Base de Gröbner, podemos ver que $r(f)$ pertence a span(Norm(I)). O resultado segue então da Afirmação 4.2.3t e do Teorema 3.1.6.

\footnotetext{
${ }^{14}$ Tal qual na seção anterior, não supomos aqui que o conjunto $G$ seja finito. Seguindo as idéias dos comentários que precedem o Teorema 4.2.31, cometeremos um abuso de linguagem ao longo desta seção e utilizaremos livremente o conjunto $G$ como paràmetro para o Algoritmo da Divisāo. Para ser preciso, entretanto, devemos ter em mente, que quando dizemos que $f$ se reduz para $f^{\prime}$ sobre $G$, queremos dizer que $f$ se reduz para $f^{\prime}$ sobre algum subconjunto finito de $G$.
} 
O seguinte lema é conseqüência da Afirmação 4.2 .34 e da presença de uma ordem admissível (ou de uma ordem parcial de semigrupo satisfazendo condição de cadeia descendente) e se encontra presente no trabalho original de Bergman. ${ }^{15}$ Dessa forma, omitiremos sua demonstração aqui.

Por outro lado, o leitor interessado em uma demonstração alternativa à dada por Bergman (e mais familiar para o contexto da Teoria de Bases de Gröbner), é convidado a verificar que ele decorre imediatamente da definição da ordem $\prec$ e do Lema 4.3.15, que serão vistos na Seção 4.3.2.

Lema 4.2.37 Seja $f$ em $K\langle X\rangle$. Então $f$ é de redução finita.

Proposição 4.2.38 Uma ambigüidade de sobreposição de $S$ é resolúvel se a relação de sobreposição associada a ela se reduz para zero sobre $G$.

Demonstração. Tome uma ambigüidade de sobreposição $s=\left(\sigma_{i_{1}}, \sigma_{i_{2}}, a, b, c\right)$ e a relação de sobreposição associada a ela $O\left(i_{1}, i_{2}, a, c\right)$. Pela definição de ambigüidade de sobreposição temos que $a W_{\sigma_{i_{1}}}=W_{\sigma i_{2}} c$. Mas então

$$
\begin{aligned}
O\left(i_{1}, i_{2}, a, c\right) & =a g_{i_{1}}-g_{i_{2}} c \\
& =a W_{\sigma_{i_{1}}}-a f_{\sigma_{i_{1}}}-W_{\sigma i_{2}} c+f_{\sigma_{i_{2}}} c \\
& =f_{\sigma_{i_{2}}} c-a f_{\sigma_{i_{1}}}
\end{aligned}
$$

Mas, por hipótese $f_{\sigma_{i_{2}}} c-a f_{\sigma_{i_{1}}}$ se reduz para zero sobre $G$. Logo, pelo Lema 4.2.35, existe uma seqüência de reduções $r$ tal que $r\left(f_{\sigma_{i_{2}}} c-a f_{\sigma_{i_{1}}}\right)=0$ e segue que $r\left(f_{\sigma_{i_{2}}} c\right)=r\left(a f_{\sigma_{i_{1}}}\right)$ do fato de $r$ ser $K$-linear por definição. Portanto, $s$ é resolúvel.

Proposição 4.2.39 Uma ambigüidade de inclusão de $S$ é resolúvel se a relação de divisão associada a ela se reduz para zero sobre $G$.

Demonstração. Seja $i=\left(\sigma_{i_{1}}, \sigma_{i_{2}}, a, b, c\right)$ uma ambigüidade de inclusão de $S$ e tome $D\left(i_{1}, i_{2}, a, c\right)$ a relação de divisão associada a ela. Então, temos

$$
\begin{aligned}
D\left(i_{1}, i_{2}, a, c\right) & =g_{i_{1}}-a g_{i_{2}} c \\
& =W_{\sigma_{i_{1}}}-f_{\sigma_{i_{1}}}-a W_{\sigma_{i_{2}}} b+a f_{\sigma_{i_{2}}} c \\
& =a f_{\sigma_{i_{2}}} c-f_{\sigma_{i_{1}}}
\end{aligned}
$$

Mas então, $a f_{\sigma_{i_{2}}} c-f_{\sigma_{i_{1}}}=D\left(i_{1}, i_{2}, a, c\right)$ se reduz para zero sobre $G$, por hipótese. Logo, pelo Lema 4.2 .35 , existe uma seqüència de reduções $r$, tal que $r\left(a f_{\sigma_{i_{2}}} c-f_{\sigma_{i_{1}}}\right)=0$, donde $r\left(a f_{\sigma_{i_{2}}} c\right)=r\left(f_{\sigma_{i_{1}}}\right)$ pela $K$-linearidade de $r$ e segue o resultado.

Voltamo-nos agora para mostrar que a condição 2 do Lema do Diamante implica o fato de que o conjunto $G$ é uma Base de Gröbner. Antes de apresentar esse resultado (na Proposição 4.2.43), rejamos alguns lemas.

\footnotetext{
${ }^{15} \mathrm{O}$ leitor encontra os detalhes dessa demonstração no Lema 2.8 de [Fer95].
} 
Lema 4.2.40 Seja $f$ um elemento de redução única de $K\langle X\rangle$ e seja $r=\left(r_{1} r_{2} \cdots r_{n}\right)$ uma seqüência finita de reduções qualquer. Então $r(f)$ é de redução única e $r_{S}(f)=r_{S}(r(f))$.

Demonstração. Claro que $r(f)$ é de redução finita, pelo Lema 4.2.37. Tome então $s=\left(s_{1} s_{2} \cdots s_{s}\right)$ uma seqüência final arbitrária em $r(f)$. Mas então $s(r(f))$ é um elemento irredutível e $s(r(f))=s_{s} s_{s-1} \cdots s_{1} r_{n} r_{n-1} \cdots r_{1}(f)$. Então a seqüência $\left(r_{1} r_{2} \cdots r_{n} s_{1} s_{2} \cdots s_{s}\right)$ é final em $f$, donde $s(r(f))=r_{S}(f)$, para toda seqüência $s$. Logo, $r(f)$ é de redução única com $r_{S}(r(f))=r_{S}(f)$.

Lema 4.2.41 Suponha que todo elemento de $K\langle X\rangle$ é de redução única. Seja agora $f$ um elemento de $K\langle X\rangle$ e $r$ uma seqüência de reduções final em $f$. Então $r(f)=f^{\prime}$ se e somente se $f$ se reduz para $f^{\prime}$ sobre $G$.

Demonstração. Se $f$ se reduz para $f^{\prime}$ sobre $G$, então existe uma seqüência de reduções final em $f r^{\prime}$ tal que $r^{\prime}(f)=f^{\prime}$, pelo Lema 4.2.35. O resultado segue então do fato de $f$ ser de redução única.

Assuma agora que existe uma seqüência de reduções $r$, final em $f$ tal que $r(f)=f^{\prime}$. Pelo Lema 4.2.35, sabemos que $\operatorname{DivisÃo}(G, f)$ é igual à imagem de $f$ por alguma seqüência de reduções $r^{\prime}$. Mas, pelo fato de $f$ ser de redução única, $r(f)=r^{\prime}(f)$ e segue o resultado.

O seguinte lema é puramente técnico e será utilizado na demonstração da Proposição 4.2.43.

Lema 4.2.42 Suponha que todo elemento de $K\langle X\rangle$ é de redução única. Nestas condições, se um elemento $f$ de $K\langle X\rangle$ é tal que $r_{S}(f)=0$, então $r_{S}(a f b)=0$, para todos a e $b$ em $X^{*}$.

Demonstração. Mostraremos por indução em $m$ que se $f$ é um elemento nas hipóteses do lema, de forma que existe uma sequiência de reduções $r=\left(r_{1} r_{2} \cdots r_{m}\right)$ final em $f$, então $r_{s}(a f b)=0$. Note que, como estamos assumindo que todo elemento é de redução única, basta exibir uma seqüência de reduções para $a f b$ com imagem zero. Denote $r_{k}=r_{a_{k} \sigma_{i_{k}} b_{k}}$, para todo $1 \leq k \leq m$. Se $f$ é nulo, não há o que mostrar; assuma portanto que $f \neq 0$ e vejamos o caso em que $m=1$.

Neste caso, $r=r_{a_{1} \sigma_{i_{1}} b_{1}}$ e $r$ atua não trivialmente em $f$. Logo, $a_{1} W_{\sigma_{i_{1}}} b_{1}$ ocorre no suporte de $f$. Com isso temos que $a a_{1} W_{\sigma_{i_{1}}} b_{1} b$ ocorre no suporte de $a f b$ e a função de redução $r^{\prime}=r_{a a_{1} \sigma_{i_{1}} b_{1} b}$ atua não trivialmente em $f$. Portanto, pela Afirmação 4.2.34:

$$
\begin{aligned}
r^{\prime}(a f b) & =a f b-\alpha a a_{1} g_{i_{1}} b_{1} b(\alpha \text { é um coeficiente não nulo }) \\
& =a\left(f-\alpha a_{1} g_{i_{1}} b_{1}\right) b \\
& =a(r(f)) b=a 0 b=0
\end{aligned}
$$

Assuma agora que $m>1$ e que para todo $f$ nas hipóteses do lema, de forma que existe uma seqüência de reduções $s=\left(s_{1} s_{2} \cdots s_{k}\right)$ final em $f$, então $r_{s}(a f b)=0$, para todo $1 \leq k<m$. 
Seja então $r=\left(r_{1} r_{2} \cdots r_{m}\right)$ uma sequiência final de reduções em $f$. Podemos assumir, sem perda de generalidade, que $r$ é efetiva. Então, $a_{1} W_{\sigma_{i_{1}}} b_{1}$ ocorre no suporte de $f$. Assim, como antes, $a a_{1} W_{\sigma_{i_{1}}} b_{1} b$ ocorre no suporte de $a f b$. Logo, definindo $r_{1}^{\prime}=r_{a a_{1} \sigma_{i_{1}} b_{1} b}$, podemos utilizar novamente a Afirmação 4.2 .34 para escrever:

$$
\begin{aligned}
r_{1}^{\prime}(a f b) & =a f b-\alpha a a_{1} g_{i_{1}} b_{1} b \text { ( } \alpha \text { é um coeficiente não nulo) } \\
& =a\left(r_{1}(f)\right) b
\end{aligned}
$$

Mas então, note que $\left(r_{2} r_{3} \cdots r_{m}\right)$ é uma seqüência final em $r_{1}(f)$ e $r_{S}\left(r_{1}(f)\right)=r_{S}(f)=0$. Podemos, portanto, utilizar a hipótese de indução e a igualdade acima para concluir que $r_{S}\left(r_{1}^{\prime}(a f b)\right)=0$. O resultado segue então do Lema 4.2.40.

Proposição 4.2.43 Todo elemento de $K\langle X\rangle$ é de redução única se e somente se $G$ é uma Base de Gröbner.

Demonstração. Denotamos por $I$ o ideal gerado por $G$ e vejamos que se todo elemento é de redução única, então $G$ é uma Base de Gröbner.

Primeiramente, vamos mostrar que todo elemento de $I$ se reduz para zero sobre $G$.

Para tanto, defina $J$ como o conjunto dos elementos de $K\langle X\rangle$ que se reduzem para zero sobre $G$. Note que, pelo Lema $4.2 .41, J$ é o seguinte conjunto

$$
J=\left\{f \in K\langle X\rangle \mid r_{S}(f)=0\right\}
$$

Vamos mostrar que $J \supseteq I$. Note primeiramente que $G \subseteq J$, uma vez que todo elemento de $G$ se reduz para zero sobre $G$ trivialmente (basta escolher ele mesmo na primeira iteração do Algoritmo da Divisão). Vejamos então que $J$ é um ideal.

Tome $f_{1}$ e $f_{2}$ em $J$ e considere o elemento $f_{1}+\alpha f_{2}$ (com $\alpha$ um escalar). Note que podemos aplicar o Algoritmo da Divisão para obter como saída um elemento irredutível de $K\langle X\rangle$. Com isso, existe uma sequiência de reduções $r$ finita e final em $f_{1}+\alpha f_{2}$. Então, pelo Lema 4.2.40 existem sequiências de reduções $r^{\prime}$ e $r^{\prime \prime}$ tais que $r^{\prime}\left(r\left(f_{1}\right)\right)=r_{S}\left(f_{1}\right)$ e $r^{\prime \prime}\left(r^{\prime}\left(r\left(f_{2}\right)\right)\right)=r_{S}\left(f_{2}\right)$. Mas então, como $r\left(f_{1}+\alpha f_{2}\right)$ é irredutível, podemos usar o fato de as funções de redução serem $K$-lineares (por definição) para obter:

$r\left(f_{1}+\alpha f_{2}\right)=r^{\prime \prime}\left(r^{\prime}\left(r\left(f_{1}+\alpha f_{2}\right)\right)\right)=r^{\prime \prime}\left(r^{\prime}\left(r\left(f_{1}\right)\right)\right)+\alpha r^{\prime \prime}\left(r^{\prime}\left(r\left(f_{2}\right)\right)\right)=r_{S}\left(f_{1}\right)+\alpha r_{S}\left(f_{2}\right)=0$

Mas como $f_{1}+\alpha f_{2}$ é de redução única, segue que $f_{1}+\alpha f_{2}$ pertence a $J$.

Para ver que $J$ é um ideal, resta mostrar que, para todo $f$ em $J$ e todos $f_{1}$ e $f_{2}$ em $K\langle X\rangle$, o elemento $f_{1} f f_{2}$ também está em $J$. Mas como sabemos que a combinação linear de elementos de $J$ pertence a $J$, basta mostrar que se $f$ pertence a $J$, então $a f b$ também pertence a $J$, para monômios quaisquer $a$ e $b$. Mas isso é precisamente o que diz o Lema 4.2 .42 . 
Portanto, $J$ é um ideal. Com isso, temos que $I \subseteq J$ (pois, $J$ é um ideal que contém os geradores de $I$ ). Mas então, o resultado segue imediatamente do Corolário 4.1.8.

A implicação reversa do enunciado da Proposição segue imediatamente do Lema 4.2.36.

\section{Demonstração do Teorema 4.2.31 (no caso em que $R=K\langle X\rangle$ ).}

Se $G$ é uma Base de Gröbner, segue que as relações de sobreposição e de divisão se reduzem para zero sobre $G$ pela Proposição 4.1 .4 e pelo Corolário 3.1.7, como antes.

Por outro lado, se assumimos que todas as relações de sobreposição e de divisão se reduzem para zero sobre $G$, podemos utilizar as Proposições 4.2 .38 e 4.2.39 para ver que todas as ambigüidades de sobreposição e de inclusão são resolúveis.

Portanto, podemos utilizar o Lema do Diamante (Teorema 4.2.33) para concluir que todo elemento de $K\langle X\rangle$ é de redução única. O resultado segue então da Proposição 4.2.43.

\subsubsection{Bases que não Admitem Ordens Admissíveis}

Esta seção tem o propósito de apresentar uma família de exemplos de bases multiplicativas que não admitem nenhuma ordem admissível. Ao fazer isso, aplicações bastante naturais para o Algoritmo da Divisão e para o Teorema 4.2.31 surgirão (na realidade, eles serão utilizados para resolver um problema de pertinência), de modo que esta seção pode ser vista como uma ilustração da teoria desenvolvida até aqui.

Iniciamos com o seguinte exemplo motivador, que serviu de inspiração para a construção da família mencionada.

Exemplo 4.2.8 Seja $X=\{a, x, y\}$ um alfabeto e considere $K\langle X\rangle$. Seja $I$ o ideal de $K\langle X\rangle$ gerado por $\{a x y a-x y a, x y-y x\}$ e $A:=K\langle X\rangle / I$. Considere ainda a projeção canônica $\pi: K\langle X\rangle \longrightarrow A$.

Note que, pelo fato de $I$ ser um ideal 2-nomial, segue da Proposição 3.4 .22 que $\mathcal{B}:=$ $\pi\left(X^{*}\right) \backslash\{0\}$ é uma base multiplicativa de $A$. Afirmamos que $\mathcal{B}$ não admite nenhuma ordem admissível.

Suponha então que temos uma ordem admissível $\leq$ fixada em $\mathcal{B}$ e veremos que isso nos leva a uma contradição. Mostraremos primeiramente que $\pi(x y)<\pi(a x y)$.

É claro que $\pi(x y) \leq \pi(a x y)$, uma vez que $\pi(x y)$ divide $\pi(a x y)$. Portanto, resta-nos mostrar que $\pi(a x y) \neq \pi(x y)$. Mas a igualdade ocorre se e somente se $a x y-x y$ pertence a $I$. Podemos então utilizar a própria Teoria de Bases de Gröbner ( $\mathrm{cm} K\langle X\rangle$ ) para ver que axy $-x y \notin I$. Assim, se tomarmos a ordem deglex em $X$, com $x>y$, vimos no Exemplo 3.2.3 que uma Base de Gröbner para $I$ é:

$$
G=\left\{a y^{i} \cdot x^{i} a-y^{i} \cdot x^{i} a, x y-y x \mid i \geq 1\right\}
$$


Agora não é difícil ver que $a x y-x y$ não se reduz para zero sobre $G$ (de fato, se reduz apenas para $a y x-y x)$. Portanto, $a x y-x y$ não pertence a $I$. Portanto, podemos concluir que $\pi(x y)$ é estritamente menor que $\pi(a x y)$.

Mas então, podemos utilizar a condição 3 da Definição 3.1.1 para concluir que

$$
\pi(a x y) \pi(a)=\pi(a x y a)<\pi(x y) \pi(a)=\pi(x y a)
$$

(podemos utilizar novamente a Base de Gröbner $G$, ou o Corolário 3.4.26 para verificar que ambos $\pi($ axya) e $\pi(x y a)$ são não nulos).

Portanto, chegamos a uma contradição com o fato de $\pi(a x y a)$ ser igual a $\pi(x y a)$, donde concluímos que não pode existir uma ordem admissível em $\mathcal{B}$.

As idéias presentes no exemplo anterior podem ser generalizadas. Para tanto, tomamos uma álgebra de caminhos $K \Gamma$ e fixamos a base $\mathcal{P}$ de $K \Gamma$. Seja ainda $I$ um ideal 2-nomial de $K \Gamma$ e $A:=K \Gamma / I$. Denote por $\pi: K \Gamma \longrightarrow A$ a projeção canônica e suponha que $I$ é tal que existem monômios $u, p$ e $q$ de $\mathcal{P}$ satisfazendo:

1. $v:=$ puq.

2. $\pi(v)$ é não nulo (note que, como $\pi(u)$ é um divisor de $\pi(v)$, essa hipótese implica que $\pi(u)$ é não nulo).

3. Uma das seguintes condições é verdadeira:

(a) $\pi(p u) \neq \pi(u)$ e $\pi(u q)=\pi(v)$.

(b) $\pi(u q) \neq \pi(u)$ e $\pi(p u)=\pi(v)$.

Podemos então mostrar que a base multiplicativa $\mathcal{B}:=\pi(\mathcal{P}) \backslash\{0\}$ de $A$ não admite nenhuma ordem admissível (claro que $\mathcal{B}$ é multiplicativa pela Proposição 3.4.22).

Vejamos o caso em que $\pi(u) \neq \pi(u q)$; a situação em que $\pi(u) \neq \pi(p u)$ segue de forma análoga. Note primeiramente, que, como $\pi(v)$ é não nulo por hipótese, segue que $\pi(u q)$ e $\pi(p u)$ são não nulos, uma vez que ambos dividem $\pi(v)$.

Assuma então que existe uma ordem admissível $\leq$ em $\mathcal{B}$ e vejamos que isso nos leva a uma contradição. Mas então, como $\pi(u)$ divide $\pi(u q)$, temos que $\pi(u)<\pi(u q)$. Logo, $\pi(p u)<\pi(p u q)=$ $\pi(v)$, nos levando a uma contradição.

Note que é possível construir exemplos satisfazendo as condições acima. No Exemplo 4.2.8, se tomarmos $u=x y$ e $p=q=a$ veremos que as condições acima mencionadas são satisfeitas.

As idéias acima podem ser utilizadas para encontrar famílias infinitas de álgebras, equipadas com bases multiplicativas que não admitem nenhuma ordem admissível. A fim de ilustrar como isso pode ser feito, apresentamos o seguinte exemplo.

Exemplo 4.2.9 Seja $X=\{a, b, x\}$ e considere $K\langle X\rangle$ com a base multiplicativa $X^{*}$. Definimos o ideal $I_{j}:=\left\langle a x^{j} b-a x^{j}\right\rangle$, para todos os valores de $j>0$. Definimos ainda, para cada $j$, a álgebra $A_{j}:=K\langle X\rangle / I_{j}$ e a projeção canônica $\pi_{j}: K\langle X\rangle \longrightarrow A_{j}$.

Note que pela Proposição $3.4 .22, \mathcal{B}_{j}:=\pi_{j}\left(X^{*}\right) \backslash\{0\}$ é uma base multiplicativa de $A_{j}$, para todo $j$. Afirmamos que, para todo $j, \mathcal{B}_{j}$ não admite ordem admissivel alguma. 
Para ver a afirmação, fixe um valor de $j$. Então, podemos utilizar o Corolário 3.4.26 para ver que a imagem de todo monômio de $X^{*}$ por $\pi_{j}$ é não nula.

Com a notação acima, tomemos $u=x^{j}, p=a$ e $q=b$. Então, $v=a x^{j} b, \pi_{j}(u)$ é não nulo e $\pi_{j}\left(a x^{j} b\right)=\pi(v)=\pi_{j}(p u)=\pi_{j}\left(a x^{j}\right)$. Pelos argumentos acima, resta-nos mostrar que $x^{j} b-x^{j}$ não pertence a $I$.

Para ver esse fato, basta notar que fixada a ordem deglex em $X^{*}(\operatorname{com} a>b>x)^{16}$ o conjunto $G:=\left\{a x^{j} b-a x^{j}\right\}$ é uma Base de Gröbner para $I$. Assim, é fácil ver que $x^{j} b-x^{j}$ não se reduz para zero sobre $G$.

$O$ fato de $\mathcal{B}_{j}$ não admitir nenhuma ordem admissível segue então dos argumentos apresentados anteriormente.

\subsection{O Procedimento de Mora}

Esta seção apresenta o Procedimento de Mora para as álgebras de caminhos. Esse procedimento foi originalmente desenvolvido por Mora [Mor94] para o contexto das álgebras livres e foi posteriormente generalizado para álgebras de caminhos [FFG93, Gre97]. Seu propósito é encontrar uma Base de Gröbner para um ideal $I$ a partir de um conjunto finito de geradores.

Porém, como visto na Seção 3.2, Bases de Gröbner podem ser infinitas e a Seção 4.4 apresentará outras restrições envolvendo a computabilidade de Bases de Gröbner. Esses dois comentários eliminam nossas esperanças de encontrar um algoritmo para calcular Bases de Gröbner no caso não comutativo. De fato, o procedimento que apresentaremos aqui pode nunca terminar sua computação. Por outro lado, algumas garantias são dadas pelo procedimento. São elas:

- Se o Procedimento de Mora terminar sua computação, ele devolve uma Base de Gröbner finita para o ideal $I$.

- Ainda que o procedimento não termine sua computação, a união (possivelmente infinita) de todos os conjuntos gerados por ele é uma Base de Gröbner para $I$.

- Se o ideal $I$ admite alguma Base de Gröbner finita (para a ordem admissível fixada), então o Procedimento de Mora termina sua computação.

Exibimos nesta seção duas versões do Procedimento de Mora. A primeira possui uma descrição mais simplificada e é muito parecida com a versão original apresentada por Mora para as álgebras livres. A segunda será vista na Seção 4.3.3. Ela está presente em algumas abordagens da teoria no contexto de álgebras de caminhos [Gre97] e faz uso do conceito de conjunto tl-reduzido (que será explorado na Seção 4.3.2).

Vale comentar que ambas as versões do procedimento possuem as propriedades acima destacadas. Contudo, a segunda versão possui a propriedade extra de produzir uma Base de Gröbner minimal.

Finalmente, na Seção t.3.t discutiremos brevemente como a Base de Gröbner reduzida para um ideal pode ser construída a partir de uma Base de Gröbner minimal (de forma algorítmica, caso essa última seja finita).

\footnotetext{
${ }^{16}$ Na realidade, as conclusōes aqui apresentadas poderiam ser obtidas utilizando deglex com qualquer ordem nas letras.
} 
O leitor atento irá recordar de nossos comentários no Capítulo 2 que muitos estudos têm sido feito no sentido de encontrar "melhorias" para o Algoritmo de Buchberger, de forma a torná-lo mais eficiente. Como comentado, tais melhorias consistem primordialmente em critérios que permitem desconsiderar certos tipos de S-polinômios e adotar diferentes estratégias de seleção de S-polinômios. Questões desse tipo também foram estudadas para o caso não comutativo (embora com menor intensidade) [Mor94, Kel97, Kel98]. Entretanto, não abordaremos estas questões neste trabalho.

Por toda a seção, tome $K \Gamma$ uma álgebra de caminhos e $\mathcal{P}$ sua base multiplicativa formada pelo conjunto de todos os caminhos em $\Gamma$. Como sempre, assumiremos que $K \Gamma$ é finitamente gerada e que temos uma ordem admissível fixada na base $\mathcal{P}$.

\subsubsection{Uma Primeira Versão}

Esta seção dedica-se a apresentar uma primeira versão para o Procedimento de Mora e provar as propriedades acima mencionadas. Iniciamos com uma descrição do procedimento no Algoritmo 4.3.1.

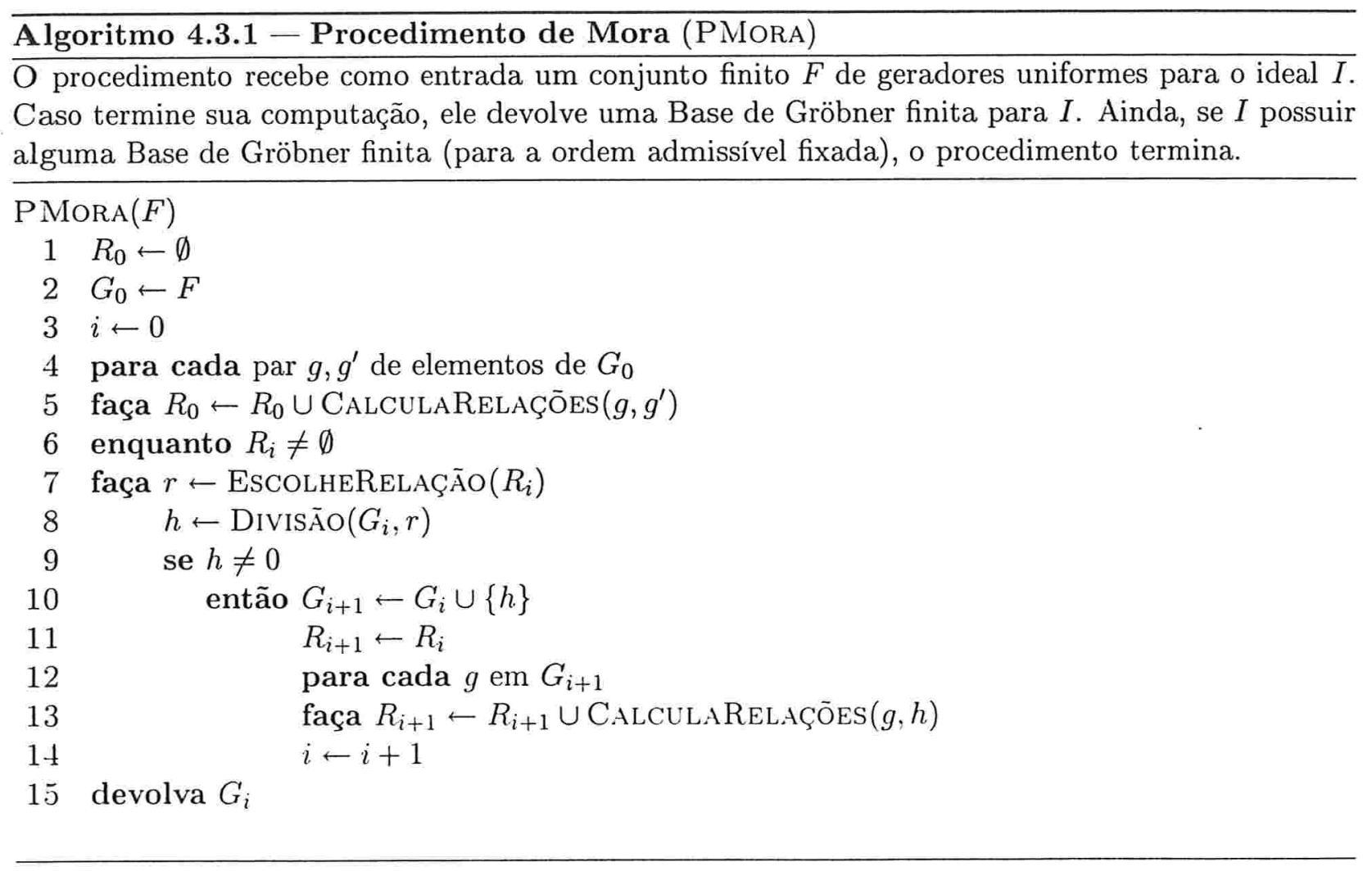

Uma observação de implementação pode ser feita neste ponto. Optamos por descrever o procedimento fazendo cópias dos conjuntos $G_{i}$ e $R_{i}$ a cada iteração, pois tal descrição nos será útil nas demonstrações que seguem. Porém, é claro que uma implementação desse procedimento poderia optar por simplesmente modificar os conjuntos $G$ e $R$ a cada iteração, em vez de fazer cópias deles.

$\mathrm{O}$ procedimento faz uso de duas funções auxiliares ainda não descritas. Fornecemos uma breve descrição de cada uma a seguir. 
CalculaRelações $\left(g, g^{\prime}\right)$ Esta função recebe como entrada dois elementos de $K \Gamma$ e devolve um conjunto contendo todas as relações de sobreposição e de divisão não nulas envolvendo os elementos $g$ e $g^{\prime}$.

No caso das álgebras de caminhos, esta função pode ser implementada sem muita dificuldade fazendo buscas por subpalavras envolvendo $\widehat{g}$ e $\widehat{g^{\prime}}$. Ademais, sua saída será sempre um conjunto finito, pela Proposição 4.2.29.

EscolheRelaÇÃo $(R)$ Esta função recebe como entrada um conjunto composto por relações de sobreposição e de divisão e devolve um desses elementos (removendo-o do conjunto original $R$ ). Ela deve possuir a seguinte propriedade: se um elemento $s$ é inserido em um conjunto $R_{i}$ e sucessivas chamadas são feitas para a função EsCOLHERELAÇÃo, $s$ deve ser devolvido em um número finito de chamadas de EscolheRelaÇão. Em outras palavras, exigimos que toda relação de sobreposição e de divisão encontrada pelo procedimento seja considerada em tempo finito. ${ }^{17}$

Essa propriedade pode ser atingida, por exemplo, implementando os conjuntos $R_{i}$ como uma única fila que sofre inserções e remoções durante a execução do procedimento.

Fornecemos a seguir duas simulações de execução dessa primeira versão do procedimento. Para as simulações, os conjuntos $R_{i}$ serão considerados como filas.

Exemplo 4.3.1 Considere o grafo $\Gamma$ dado na figura abaixo.

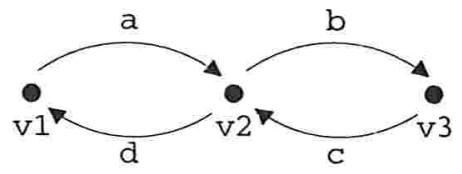

Tome a álgebra de caminhos $K \Gamma$ e considere a ordem de grau e lexicografia com $a>b>$ $c>d$. Seja então $F=\left\{g_{1}, g_{2}, g_{3}\right\}$, onde $g_{1}:=c d a b-c b, g_{2}:=b c-d a$ e $g_{3}:=c d a d a b-c b$ e seja $I:=\langle F\rangle$. Note que os elementos $g_{1}, g_{2}$ e $g_{3}$ são todos uniformes. Vejamos uma simulação de uma chamada para PMIORA $(F)$.

Inicialmente, o procedimento copia $F$ para $G_{0}$ e encontra as relações de divisão e de sobreposição envolvendo elementos de $G_{0}$. Claramente, não existem relações de divisão entre os elementos de $G_{0}$, mas existem as seguintes relações de sobreposição:

$$
\begin{aligned}
& s_{1}=O\left(g_{2}, g_{1}, c d a, c\right)=c d a g_{2}-g_{1} c=-c d a d a+c b c \\
& s_{2}=O\left(g_{1}, g_{2}, b, d a b\right)=b g_{1}-g_{2} d a b=\text { dadab }-b c b \\
& s_{3}=O\left(g_{3}, g_{2}, b, \text { dad } a b\right)=b g_{3}-g_{2} \text { dad } a b=(d a)^{3} b-b c b \\
& s_{4}=O\left(g_{2}, g_{3}, c d a d a, c\right)=c d a d a g_{2}-g_{3} c=-c(d a)^{3}+c b c
\end{aligned}
$$

Com isso, imediatamente antes da primeira execução da linha 6, teremos:

\footnotetext{
${ }^{17}$ Note que o único lugar em que um elemento pode ser removido de um conjunto $R_{1}$ é dentro da função ESCOLHERELAÇÃOO.
} 


$$
\begin{aligned}
& G_{0}=\left\{g_{1}, g_{2}, g_{3}\right\} \\
& R_{0}=\left(s_{1}, s_{2}, s_{3}, s_{4}\right)
\end{aligned}
$$

O procedimento considera então $s_{1}$ :

$$
s_{1} \Rightarrow_{G_{0}}-c d a d a+c d a:=g_{4}
$$

Assim,

$$
G_{1} \leftarrow\left\{g_{1}, g_{2}, g_{3}, g_{4}\right\}
$$

Existe ainda a seguinte relação de divisão envolvendo $g_{4}$ e os elementos de $G_{1}$ :

$$
d_{1}=D\left(g_{3}, g_{4}, v_{3}, b\right)=g_{3}+g_{4} b=c d a b-c b
$$

e a seguinte relação de sobreposição:

$$
s_{5}=O\left(g_{4}, g_{2}, b, d a d a\right)=b g_{4}+g_{2} d a d a=-(d a)^{3}+b c d a
$$

Com isso,

$$
R_{1} \leftarrow\left(s_{2}, s_{3}, s_{4}, d_{1}, s_{5}\right)
$$

Temos uma nova iteração e o procedimento considera $s_{2}$ :

$$
\begin{aligned}
& s_{2} \Rightarrow_{G_{1}} \text { dadab }-d a b:=g_{5} \\
& G_{2} \leftarrow\left\{g_{1}, g_{2}, \ldots, g_{5}\right\}
\end{aligned}
$$

Existe ainda uma relação de divisão e duas relações de sobreposição envolvendo $g_{5}$ e os elementos de $G_{2}$ :

$$
\begin{aligned}
& d_{2}=D\left(g_{3}, g_{5}, c, v_{3}\right)=c d a b-c b \\
& s_{6}=O\left(g_{2}, g_{5}, \text { dada, } c\right)=d a d a g_{2}-g_{5} c=-(d a)^{3}+d a b c \\
& s_{7}=O\left(g_{5}, g_{4}, c, b\right)=c g_{5}+g_{4} b=0
\end{aligned}
$$

Portanto,

$$
R_{2} \leftarrow\left(s_{3}, s_{4}, d_{1}, s_{5}, d_{2}, s_{6}\right)
$$

Nas très iterações seguintes, o procedimento considera $s_{3}, s_{4}$ e $d_{1}$. Contudo, todos eles se reduzem para zero sobre $G_{2}$, de forma que as linhas 10 a 14 não são executadas durante essas iterações. Logo $i$ não é modificado e nenhum novo conjunto é gerado.

Então o procedimento considera $s_{5}$ :

$$
\begin{aligned}
& s_{5} \Rightarrow G_{G_{2}}-(d a)^{3}+(d a)^{2}:=g_{6} \\
& G_{3} \leftarrow\left\{g_{1}, g_{2}, \ldots, g_{6}\right\}
\end{aligned}
$$


Não existem relações de divisão envolvendo $g_{6}$ e os elementos de $G_{3}$. Porém, existem as seguintes relações de sobreposição:

$$
\begin{aligned}
& s_{8}=O\left(g_{6}, g_{4}, c, d a\right)=c g_{6}-g_{4} d a=0 \\
& s_{9}=O\left(g_{5}, g_{6}, d a, b\right)=d a g_{5}+g_{6} b=0 \\
& s_{10}=O\left(g_{6}, g_{6}, d a, d a\right)=d a g_{6}-g_{6} d a=0 \\
& s_{11}=O\left(g_{6}, g_{6},(d a)^{2},(d a)^{2}\right)=(d a)^{2} g_{6}-g_{6}(d a)^{2}=0
\end{aligned}
$$

Logo,

$$
R_{3}=\left(d_{2}, s_{6}\right)
$$

Finalmente o processo considera os elementos $d_{2}$ e $s_{6}$ nas duas iterações seguintes. Porém, como ambos se reduzem para zero sobre $G_{3}$, nenhum novo conjunto é gerado.

O processo termina devolvendo:

$$
G_{3}=\left\{g_{1}, \ldots, g_{6}\right\}
$$

$\Delta$

O exemplo a seguir ilustra uma situação em que o Procedimento de Mora não termina sua computação.

Exemplo 4.3.2 Para este exemplo, considere $X=\{x, y\}$, fixe a ordem de grau e lexicografia com $x>y$ em $X^{*}$ e tome a álgebra livre $K\langle X\rangle$. Seja então $I$ o ideal gerado por $F:=\left\{g_{0}\right\}, \operatorname{com} g_{0}:=x^{2}-x y$. Vejamos uma simulação para a chamada PMorA $(F)$. Inicialmente $G_{0} \leftarrow\left\{g_{0}\right\}$. Claro que não existem relações de divisão envolvendo elementos de $G_{0}$. Mas existem relações de sobreposição:

$$
s_{1}=O\left(g_{0}, g_{0}, x, x\right)=x g_{0}-g_{0} x=-x^{2} y+x y x
$$

Portanto,

$$
R_{0}=\left(s_{1}\right)
$$

Assim, na primeira execução do laço das linhas 6 a 14, teremos:

$$
s_{1} \Rightarrow G_{0} x y x-x y^{2}:=g_{1}
$$

Logo,

$$
G_{1} \leftarrow\left\{g_{0}, g_{1}\right\}
$$

e teremos as seguintes relaçōes de sobreposição envolvendo $g_{1}$ e os elementos de $G_{1}$ :

$$
\begin{aligned}
& s_{2}=O\left(g_{0}, g_{1}, x y, x\right)=x y g_{0}-g_{1} x=-x y x y+x y^{2} x \\
& s_{3}=O\left(g_{1}, g_{0}, x, y x\right)=x g_{1}-g_{0} y x=-x^{2} y^{2}+x y^{2} x \\
& s_{4}=O\left(g_{1}, g_{1}, x y, y x\right)=x y g_{1}-g_{1} y x=-x y x y^{2}+x y^{3} x
\end{aligned}
$$


Dado um inteiro $h$ maior ou igual a zero, podemos definir o elemento $g_{h}:=x y^{h} x-x y^{h+1}$. Tome dois inteiros $i$ e $j$ maiores ou iguais a zero e considere $g_{i}$ e $g_{j}$. Então teremos a seguinte relação de sobreposição envolvendo $g_{i}$ e $g_{j}$ :

$$
O\left(g_{j}, g_{i}, x y^{i}, y^{j} x\right)=x y^{i} g_{j}-g_{i} y^{j} x=-x y^{i} x y^{j+1}+x y^{i+j+1} x
$$

Calculando a redução dessa relação sobre qualquer conjunto que contenha $g_{i}$ e $g_{j}$, chegamos a

$$
-x y^{i} x y^{j+1}+x y^{i+j+1} x+g_{i} y^{j+1}=x y^{i+j+1} x-x y^{i+j+2}=g_{i+j+1}
$$

É claro que $x y^{i+j+1} x$ não é divisível por $\widehat{g_{h}}$, para nenhum $h \neq i+j+1$. Assim, se denotarmos por $G$ a união de todos os conjuntos $G_{i}$ produzidos pelo procedimento, vemos que todos os elementos da forma $g_{h}$, para $h \geq 0$ pertencem a $G$.

Ademais, dados dois elementos $g_{i}$ e $g_{j}$ todas as relações de sobreposição entre eles são da forma acima. Note também que não existem relações de divisão entre $g_{i}$ e $g_{j}$. Com isso, concluímos que

$$
G=\left\{x y^{h} x-x y^{h+1} \mid h \geq 0\right\}
$$

e o procedimento nunca terminará sua computação. ${ }^{18}$ ॥

O próximo exemplo mostra a razão para exigirmos que todo elemento inserido em algum $R_{i}$ seja devolvido em tempo finito pela função EscolheRELAÇÃo. No exemplo, o procedimento nunca termina sua computação, apesar de o ideal possuir uma Base de Gröbner finita. A razão para tanto é o adiamento indeterminado de certa computação.

Exemplo 4.3.3 Tome o alfabeto $X=\{a, b, c, d, e, f\}$ e considere $K\langle X\rangle$. Fixe a ordem de peso e lexicografia sobre $X^{*}$ com a seguinte aplicação peso:

$$
\begin{aligned}
& \phi(a)=3 \\
& \phi(b)=\phi(c)=\phi(d)=\phi(e)=\phi(f)=1
\end{aligned}
$$

Tome ainda a seguinte ordem nas letras de $X: a<b<c<d<e<f$. Com isso, temos uma ordem admissível em $X^{*} \cdot{ }^{19}$

Seja então $F=\left\{g_{1}, g_{2}, g_{3}, g_{4}, g_{5}, g_{6}, g_{7}\right\}$, com:

$$
\begin{array}{lll}
g_{1}:=c a-a c & g_{2}:=d a-a d & g_{3}:=b a-b^{2} c \\
g_{1}:=b e^{2}-b^{2} c & g_{5}:=b^{2} c f-b c & g_{6}:=e^{2} f-b c^{2} \\
g_{7}:=b c d & &
\end{array}
$$

Claro que todos os elementos de $F$ são uniformes por se tratar de uma álgebra livre. Podemos então simular a execução de uma chamada PMorA $(F)$. Assuma entretanto,

\footnotetext{
${ }^{18}$ Não é difícil ver que $G$ é a Base de Gröbner reduzida para $I$. Logo, por meio do Corolário 3.2.15, concluímos que $I$ não possui nenhuma Base de Gröbner finita (com respeito à ordem fixada).

${ }^{19}$ A definição da ordem de peso e lexicografia pode ser vista na Seção 3.1 .
} 
que a função ESCOLHERELAÇÃo devolve sempre a última relação inserida (como uma pilha).

Com isso, $G_{0} \leftarrow\left\{g_{1}, g_{2}, g_{3}, g_{4}, g_{5}, g_{6}, g_{7}\right\}$ e temos apenas duas relações de sobreposição envolvendo elementos de $G_{0}$ (não há relações de divisão):

$$
\begin{aligned}
& s_{1}=O\left(g_{2}, g_{7}, b c, a\right)=b c g_{2}-g_{7} a=-b c a d \\
& s_{2}=O\left(g_{6}, g_{4}, b, f\right)=b g_{6}-g_{4} f=b^{2} c f-b^{2} c^{2}
\end{aligned}
$$

Considere, dessa forma, que $R_{0} \leftarrow\left(s_{1}, s_{2}\right)$.

O procedimento considera inicialmente $s_{1}$, obtendo:

$$
s_{1} \Rightarrow_{G_{0}}-b^{2} c^{2} d:=g_{8}
$$

Assim, $G_{1} \leftarrow\left\{g_{1}, g_{2}, g_{3}, g_{4}, g_{5}, g_{6}, g_{7}, g_{8}\right\}$. Mas então, teremos uma nova relação de sobreposição envolvendo $g_{8}$ e elementos de $G_{0}$ (não existem relações de divisão):

$$
s_{3}=O\left(g_{2}, g_{8}, b^{2} c^{2}, a\right)=b^{2} c^{2} g_{2}-g_{8} a=-b^{2} c^{2} a d
$$

Assim, $R_{1} \leftarrow\left(s_{3}, s_{1}\right)$ e o procedimento considerará $s_{3}$ na iteração seguinte.

Contudo, se tomarmos $k$ um inteiro maior que zero e definirmos

$$
h_{k}:=-b^{k} c^{k} d
$$

não é difícil ver que existe uma única relação de sobreposição entre $h_{k}$ e os elementos de $F \cup\left\{h_{j} \mid 0<j \leq k\right\}$ :

$$
O\left(g_{2}, h_{k}, b^{k} c^{k}, a\right)=b^{k} c^{k} g_{2}+h_{k} a=-b^{k} c^{k} a d
$$

Note que, $h_{2}=g_{8}$, que foi considerado pelo procedimento e inserido em $G_{1}$. Além disso, temos a seguinte afirmação.

Afirmação 4.3.1 Toda relação de sobreposição da forma $s=O\left(g_{2}, h_{k}, b^{k} c^{k}, a\right)$ se reduz para $-b^{k+1} c^{k+1} d$ sobre $F \cup\left\{h_{2}, h_{3}, \ldots, h_{k}\right\}$, para todo $k \geq 2$.

Demonstração. A prova segue por indução em $k$. O caso em que $k=2$ já foi visto acima quando consideramos a relação de sobreposição $s_{1}$.

Suponha, portanto, $k>2$ e o resultado válido para valores menores que $k$.

Mas então:

$$
s=O\left(g_{2}, h_{k}, b^{k} c^{k}, a\right)=b^{k} c^{k} g_{2}+h_{k} a=-b^{k} c^{k} a d
$$

E podemos reduzir $s$ por $g_{1} k$ vezes, obtendo $-b^{k} a c^{k} d$. Em seguida, podemos reduzir a resultado por $g_{3}$, chegando $a-b^{k+1} c^{k+1} d$. Claro que esse último monòmio não é divisível por nenhum elemento de $F \cup\left\{h_{2}, h_{3}, \ldots, h_{k}\right\}$, o que demonstra o resultado. 
Com isso, se o procedimento extrai de $S_{i}$ sempre a última relação de sobreposição inserida, vemos que ele calculará elementos $h_{k}$ e relações da forma $O\left(g_{2}, h_{k}, b^{k} c^{k}, a\right)$ indefinidamente, sem nunca considerar $s_{2}$. Portanto, o procedimento nunca terminará sua computação.

Por outro lado, se o procedimento considerasse $s_{2}$ em algum tempo finito, teríamos:

$s_{2} \Rightarrow_{G_{i}}-b^{2} c^{2}+b c:=g\left(G_{i}\right.$ é qualquer conjunto produzido pelo procedimento, com $i \geq 0$ )

Ainda, as seguintes relações de sobreposição envolvendo $g$ e os elementos de $F$ seriam computadas (novamente, não existem relações de divisão envolvendo esses elementos):

$$
r_{1}=O\left(g_{1}, g, b^{2} c, a\right)=b^{2} c g_{1}+g a=-b^{2} c a c+b c a
$$

Ainda, se a relação $r_{1}$ for considerada em algum tempo finito, obteremos:

$r_{1} \Rightarrow_{G_{i}} 0$ (onde $G_{i}$ é qualquer conjunto gerado pelo procedimento que contenha $F$ e $g$ )

Finalmente, se $g$ pertence a $G_{i}$, para algum $i$, então toda relação de sobreposição da forma $O\left(g_{2}, h_{k}, b^{k} c^{k}, a\right)$ se reduz para zero sobre $G_{i}$ (note que $\widehat{g}$ divide $-b^{k+1} c^{k+1} d$ ). Portanto, se a relação $s_{2}$ for considerada em algum tempo finito, o procedimento terminará sua computação.

De fato, podemos utilizar o Teorema 4.2.31 para verificar que o conjunto $F \cup\{g\}$ é uma Base de Gröbner para $\langle F\rangle$.

Iniciamos com um lema que mostra que laço das linhas 4 e 5 não oferece nenhum problema para o término do procedimento. Ele é conseqüência imediata da efetividade da função Calcularelaçōes e do fato de $G_{0}=F$ ser finito.

Lema 4.3.2 O laço das linhas 4 a 5 termina em um número finito de passos.

Uma inspeção do procedimento, os comentários feitos acerca das funções CALculaRelações e EscolheRELAÇÃo e as Proposições 4.1.13 e 4.1.2 nos permitem concluir o seguinte resultado.

Proposição 4.3.3 Dados um corpo $K$ e uma ordem admissivel $\leq$ computáveis, cada passo do procedimento PMORA é efetivo em $K \Gamma{ }^{20}$

Prosseguimos então com a demonstração da correção do Procedimento de Mora.

Lema 4.3.4 No início de cada execução da linha 6 do procedimento PMORA, os seguintes invariantes são válidos:

1. O conjunto $R_{i}$ contém todas as relações de sobreposição e de divisão envolvendo elementos de $G_{i}$ que nào se reduzem para zero sobre $G_{i}$.

\footnotetext{
${ }^{20}$ As definições de corpos e ordens computáveis podem ser vistas na Seção 4.1.2.
} 
2. Os elementos de $G_{i}$ são todos uniformes.

3. O conjunto $G_{i-1}$ está contido em $G_{i}$.

4. Os conjuntos $G_{i}$ e $R_{i}$ são finitos.

5. O ideal gerado por $G_{i}$ é igual ao ideal gerado por $F$.

\section{Demonstração.}

Os invariantes 3, 4 e 5 são de verificação imediata se utilizarmos a Proposição 4.2.29.

Vejamos agora o primeiro invariante. Uma simples inspeção do laço das linhas 4 e 5 nos mostra que ele é trivialmente verdadeiro no início da primeira execução da linha 6 .

Suponha agora que o invariante 1 seja válido no início da $j$-ésima execução da linha 6 (e que não se trata da última execução dessa linha). Mas então, ou $h=0$ e os conjuntos $G_{i}$ e $R_{i}$ não são modificados ou $i$ é incrementado e $h$ é inserido em $G_{i}$. Mas nesse caso, o laço das linhas 12 e 13 cuida de adicionar a $R_{i}$ as relações de sobreposição e de divisão envolvendo $h$ (as que não envolvem $h$ pertencem a $R_{i}$ pela hipótese de indução e pela cópia realizada na linha 11). Portanto, o invariante será válido no início da $(j+1)$-ésima execução da linha 6 .

Para o segundo invariante, note que, como o conjunto inicial $F$ é uniforme, cada relação de sobreposição e de divisão envolvendo elementos de $F$ é uniforme, pelos Lemas 4.2.6 e 4.2.7. Logo, suas reduções sobre $G_{0}=F$ serão elementos uniformes pelo Lema 4.2.8. Utilizando esses mesmos lemas e um argumento indutivo, não é difícil verificar que a propriedade se manterá no início de cada execução da linha 6 .

Lema 4.3.5 O procedimento PMora termina sua computação se e somente se $G_{j}$ é uma Base de Gröbner finita para $\langle F\rangle$, para algum $j$.

Demonstração. Se o procedimento pára, então o resultado segue dos invariantes do Lema 4.3.4 e do Teorema 4.2.31.

Por outro lado, se $G_{j}$ é uma Base de Gröbner, para algum $j$, então todas as relações de sobreposição e de divisão envolvendo elementos de $G_{j}$ se reduzem para zero sobre $G_{j}$, pelo Teorema 4.2.31. Mas, pelo invariante 3 do Lema 4.3.4, todas as relações em $R_{j}$ são relaçōes envolvendo elementos de $G_{j}$. Logo, o procedimento terminará sua computação.

Para uma melhor formalização do que segue, convencionamos que se o procedimento PMorA termina sua computação com $i=k$, então $G_{j}:=G_{k}$, para todo $j>k$. Com isso, definimos o seguinte conjunto:

$$
G_{x}:=\bigcup_{j=0}^{\infty} G_{j}
$$

Proposição 4.3.6 O conjunto $G_{\infty}$ é uma Base de Gröbner para $\langle F\rangle$. 
Demonstração. Note primeiramente que é conseqüência imediata dos invariantes do Lema 4.3.4 que $\left\langle G_{\infty}\right\rangle=\langle F\rangle$. Ainda, pelo mesmo lema, os elementos de $G_{\infty}$ são todos uniformes. Portanto, resta-nos mostrar que todas as relações de sobreposição e de divisão envolvendo elementos de $G_{\infty}$ se reduzem para zero sobre algum subconjunto finito de $G_{\infty}$

Para tanto, tome $s$ uma relação (de sobreposição ou de divisão) envolvendo elementos $g$ e $g^{\prime}$ de $G_{\infty}$. Logo, segue da definição de $G_{\infty}$ e dos invariantes do procedimento que ambos $g$ e $g^{\prime}$ pertencem a $G_{j}$, para algum $j$.

Assim, pelo primeiro invariante do Lema 4.3.4, temos duas possibilidades: $s$ se reduz para zero sobre $G_{j} \subseteq G_{\infty}$ e não há mais o que mostrar, ou $s$ não se reduz para zero sobre $G_{j}$. Mas, neste caso, $s$ pertence a $R_{j}$ e a função EscolheRELAÇÃo devolverá $s$ em algum tempo finito.

Logo, existe uma iteração $k$ (tal que o valor de $i$ é $l$ ) em que $\operatorname{DiviSÃo}\left(G_{l}, s\right)$ será calculada, devolvendo um elemento $f$. Caso $f$ seja não nulo, esse será inserido em $G_{l+1}$ e, portanto, $s$ se reduz para zero sobre $G_{l+1} \subseteq G_{\infty}$. O resultado segue então do Teorema 4.2.31.

Com isso, podemos demonstrar o seguinte teorema.

Teorema 4.3.7 Seja $F$ um subconjunto finito e uniforme de KГ. Então $\langle F\rangle$ possui uma Base de Gröbner finita se e somente se PMORA $(F)$ termina sua computação, devolvendo uma tal Base de Gröbner.

Demonstração. Pelo Lema 4.3 .5 é claro que basta mostrar que se o ideal $I:=\langle F\rangle$ possui uma Base de Gröbner finita, então o procedimento PMora termina sua computação.

Seja então $H=\left\{h_{1}, h_{2}, \ldots, h_{n}\right\}$ uma Base de Gröbner finita para $I$. Pela Proposição 4.3.6, sabemos que $G_{\infty}$ é uma Base de Gröbner para $I$. Portanto, existem $g_{1}, g_{2}, \ldots, g_{n}$ em $G_{\infty}$ tais que $\widehat{g}_{i}$ divide $\widehat{h}_{i}$, para $1 \leq i \leq n$.

É claro que $\left\{g_{1}, g_{2}, \ldots, g_{n}\right\}$ é uma Base de Gröbner para $I$. Por outro lado, não é difícil ver a partir da definição de $G_{\infty}$ e dos invariantes do procedimento que existe $j$, tal que $\left\{g_{1}, g_{2}, \ldots, g_{n}\right\} \subseteq G_{j}$. Portanto, $G_{j}$ é uma Base de Gröbner finita para $I$. Com isso, o procedimento pára pelo Lema 4.3.5.

\subsubsection{Tl-Redução}

Conforme comentado na introdução da seção, apresentaremos uma segunda versão para o Procedimento de Mora na Seção 4.3.3. O conceito de conjunto tl-reduzido é fundamental para a apresentação dessa segunda versão do Procedimento de Mora e ele será explorado aqui. Iniciamos com sua definição.

Definição 4.3.1 (TL-Redução) Dizemos que um subconjunto $G$ de $K \Gamma$ é tl-reduzido se não existem dois elementos distintos de $G, g_{1}$ e $g_{2}$, tais que $\widehat{g_{1}}$ divide $\widehat{g_{2}} \cdot{ }^{21}$

\footnotetext{
${ }^{21} \mathrm{O}$ prefixo " $\mathrm{tl}$ " vem de "termo lider".
} 
No que segue, apresentamos o algoritmo TLREduz (Algoritmo 4.3.3), que recebe como entrada um conjunto gerador finito para um ideal e devolve um conjunto gerador tl-reduzido para o mesmo ideal.

Antes, porém, é importante ressaltar que no caso de ideais finitamente gerados, é sempre possível encontrar um conjunto gerador finito de forma que os suportes de seus elementos são dois a dois distintos. Para tanto, apresentamos o Algoritmo 4.3.2.

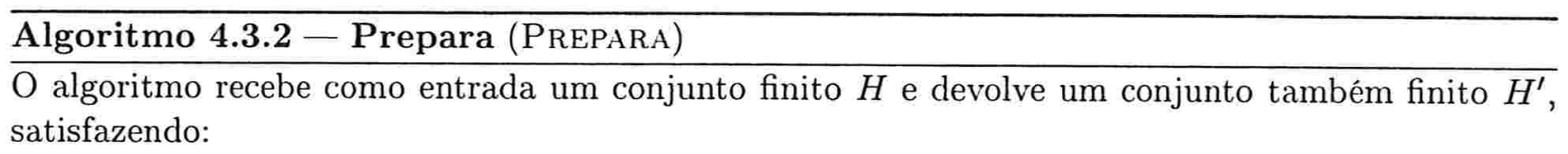

1. O ideal gerado por $H$ é igual ao ideal gerado por $H^{\prime}$.

2. Se $H$ é uniforme, então $H^{\prime}$ é uniforme.

3. Se $g$ e $g^{\prime}$ pertencem a $H^{\prime}$, então $\operatorname{supp}(g) \neq \operatorname{supp}\left(g^{\prime}\right)$.

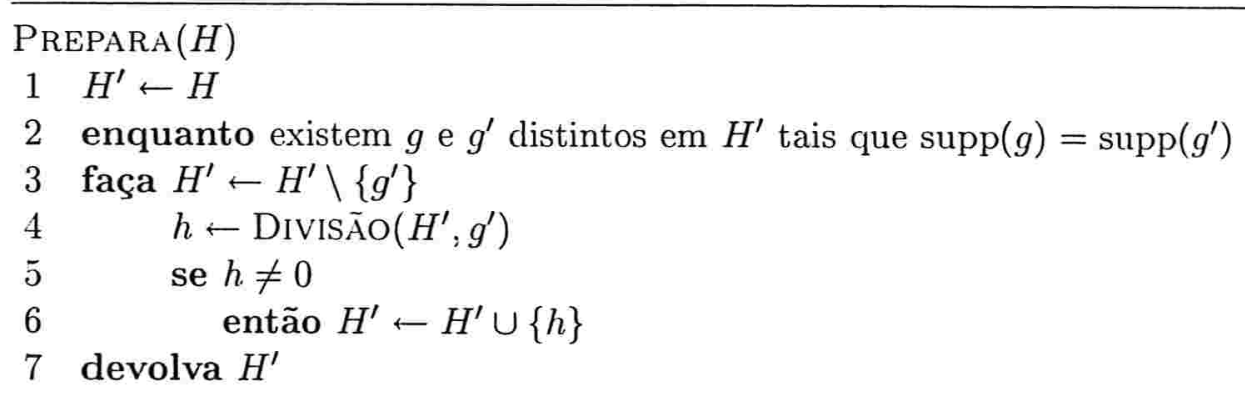

O seguinte lema nos mostra a correção do algoritmo PREPARA.

Lema 4.3.8 O algoritmo PREPARA termina sua computação em um número finito de passos $e$ devolve um conjunto $H^{\prime}$ com as propriedades desejadas.

Demonstração. As propriedades 1 e 2 da saída do algoritmo podem ser verificadas sem dificuldade, fazendo uso do Corolário 4.1 .3 e do Lema 4.2.8. A propriedade 3 é de verificação imediata.

Por outro lado, afirmamos que, a cada iteração, o suporte de $h$ é diferente do suporte de todos os elementos de $H^{\prime}$. Tal afirmação pode ser justificada pelo seguinte: se existe $g$ em $H^{\prime}$ tal que $\operatorname{supp}(g)=\operatorname{supp}(h)$, então, $\widehat{g}=\widehat{h}$. Mas isso nos leva a uma contradição com a Proposição 4.1 .2 pelo fato de $h$ ser o resultado de uma redução sobre $H^{\prime}$.

Com isso, a cada iteração, o número de elementos em $H^{\prime}$ com o mesmo suporte diminui e o resultado segue por um argumento indutivo.

Podemos agora apresentar o algoritmo que encontra um conjunto gerador tl-reduzido para um ideal, a partir de um conjunto gerador finito (Algoritmo 4.3.3).

Em seguida, dedicaremo-nos a provar a correção do algoritmo TLREDUz e algumas de suas propriedades que serão fundamentais para a segunda versão do Procedimento de Mora. 


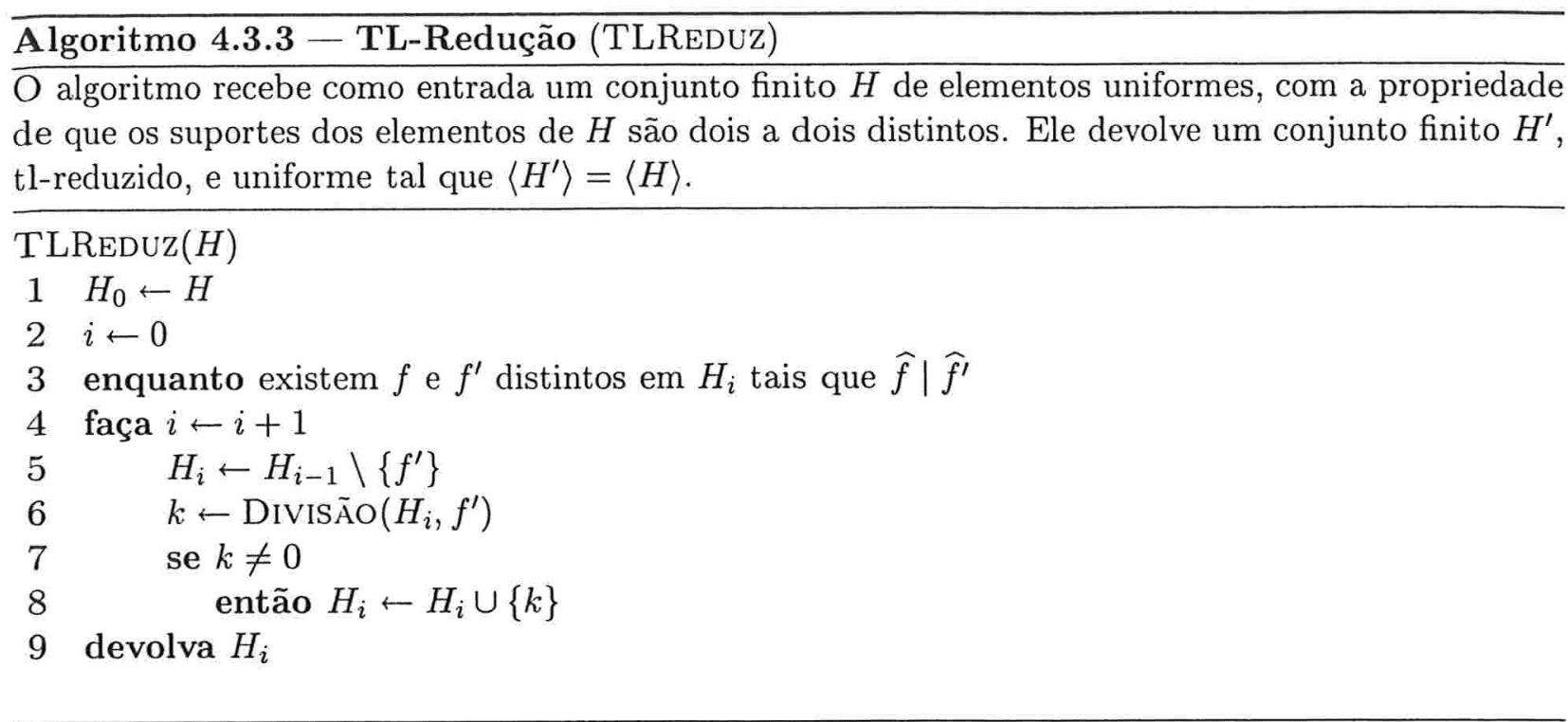

No que segue, toda referência a um conjunto $H_{i}$ deve ser vista como uma referência ao valor de $H_{i}$ no início da $(i+1)$-ésima execução da linha 3 do algoritmo, ou seja, seu valor após o término da $i$-ésima iteração do algoritmo.

No estilo do que fizemos na demonstração da primeira versão do procedimento, convencionamos que se o processo TLREDUz termina sua computação com $i=k$, então $H_{j}:=H_{k}$, para todo $j>k$. Então definimos:

$$
H_{\infty}:=\bigcup_{j=0}^{\infty} H_{j}
$$

Mostraremos primeiramente que o processo descrito acima termina sua computação em um número finito de passos.

Em linhas gerais, isso será feito definindo uma boa ordem entre os elementos da sequiência $\left(H_{i}\right)_{i \geq 0}$ e mostrando que ela decresce. Mas para tanto, precisaremos do fato de que em $H_{\infty}$ não existem dois elementos com mesmo suporte; ele será demonstrado no Lema 4.3.14.

A seguinte afirmação é conseqüência imediata da Proposição 4.1.2 e será usada recorrentemente nas demonstrações de correção do algoritmo TLREDUz.

Afirmação 4.3.9 A cada execução da linha 6 do algoritmo TLREDUZ, se $k \neq 0, \widehat{k}$ não é divisível por $\widehat{h}$, para nenhum $h$ em $H_{i}$. Em particular, $\widehat{k} \neq \widehat{h}$ e $\operatorname{supp}(k) \neq \operatorname{supp}(h)$, para todo $h$ em $H_{i}$.

Apresentamos uma conseqüência do Lema 4.2 .8 que será usada livremente adiante.

Afirmação 4.3.10 No início de cada execução da linha 3 do algoritmo TLREduz, $H_{i}$ é um conjunto de elementos uniformes.

O próximo lema é conseqüiencia imediata da Proposição 4.1.2, do Lema 4.1.7 e da condição presente na linha 3 do algoritmo. 
Lema 4.3.11 A cada execução da linha $6, \widehat{k}<\widehat{f}^{\prime}$, se $k \neq 0$.

Lema 4.3.12 Seja $f$ um elemento de $H_{h}$, para algum $h$. Então, para todo $j \geq h$, existe $f^{\prime}$ em $H_{j}$, tal que $\widehat{f}^{\prime}$ divide $\widehat{f}$.

Demonstração. A prova segue por indução em $j-h$. Mostraremos que a propriedade descrita no lema é um invariante do algoritmo para todo $i \geq h$.

Primeiramente, se $i=h$ e estamos no início da $(h+1)$-ésina execução da linha 3 , o resultado é trivialmente verdadeiro, uma vez que $f \in H_{h}$.

Suponha agora o resultado verdadeiro no início de uma execução da linha 3 (que não a última) em que $i=j \geq h$ e vejamos que ele se mantém válido no início da próxima execução desta linha. Então existe $f^{\prime}$ em $H_{j}$ tal que $\widehat{f}^{\prime} \mid \widehat{f}$, por hipótese de indução. Durante a execução do laço das linhas 3 a $8, f^{\prime}$ pode ser mantido em $H_{j+1}$ ou pode ser substituído pelo valor de $k$ calculado na linha 6 . Se ele for mantido, o resultado está demonstrado. Por outro lado, se ele for substituído, a condição da linha 3 nos mostra que existe $f^{\prime \prime}$ em $H_{j}$ de forma que $\widehat{f^{\prime \prime}} \mid \widehat{f}^{\prime}$. Pelo funcionamento do algoritmo é claro que $f^{\prime \prime}$ pertence a $H_{j+1}$ e segue o resultado.

Lema 4.3.13 O algoritmo TLREDUZ possui o seguinte invariante: no início de cada execução da linha $3, H_{i}$ é tal que os suportes de seus elementos são dois a dois distintos.

Demonstração. O invariante é trivialmente válido no início da primeira execução da linha 3 , pois $H=H_{0}$ possui essa propriedade.

Suponha agora que no início da $j$-ésima execução da linha 3 não existem dois elementos distintos em $H_{i}$ com mesmo suporte e que não se trata da última execução dessa linha. Mostraremos então que o mesmo vale para $H_{i}$ no início da $(j+1)$-ésima execução da linha 3.

Mas durante a iteração, apenas um elemento é removido de $H_{i}$ (o que naturalmente não afeta a propriedade) e $k$ é inserido. O resultado segue então da Afirmação 4.3.9.

Lema 4.3.14 Sejam $g$ e $g^{\prime}$ em $H_{\infty}$. Então $\operatorname{supp}(g) \neq \operatorname{supp}\left(g^{\prime}\right)$.

Demonstração. Tome $g$ e $g^{\prime}$ em $H_{\infty}$. Pelo Lema 4.3.13, se ambos pertencem a $H_{j}$, para algum $j$, então seus suportes são distintos. Assuma então que eles não pertencem a um mesmo $H_{j}$ para nenhum $j$, mas que $\operatorname{supp}(g)=\operatorname{supp}\left(g^{\prime}\right)$. Vejamos que isso nos leva a uma contradição.

Note primeiramente que, como seus suportes são iguais, $\widehat{g}=\widehat{g^{\prime}}$. Ainda, podemos assumir, sem perda de generalidade, que $g \in H_{n}$ e $g^{\prime} \in H_{m}$, com $n<m$. Ademais, $g \notin H_{m}$ e $g^{\prime} \notin H_{n}$. Mas então, existe $n<v \leq m$ tal que $g^{\prime} \notin H_{v-1}$, mas $g^{\prime} \in H_{v}$. Portanto, na $v$-ésima iteração do algoritmo, o valor de $k$ é $g^{\prime}$. Ainda, pelo Lema 4.3 .12 , existe $h \in H_{v-1}$ tal que $\widehat{h}$ divide $\widehat{g}$. Então temos dois casos para o valor da variável $f^{\prime}$ durante esta iteração:

- O valor de $f^{\prime}$ nāo é $h$.

Neste caso, $\widehat{h}$ divide $\widehat{g}=\widehat{g^{\prime}}=\widehat{k}$, uma contradição com a Afirmação 4.3.9. 
- O valor de $f^{\prime}$ é $h$.

Neste caso, $g^{\prime}$ é o resultado de uma redução de $h$ por $H_{v-1}$. Mas então, do fato de que $\widehat{h} \mid \widehat{g}=\widehat{g^{\prime}}$ e da Proposição 4.1.2, concluímos que $\widehat{f}^{\prime}=\widehat{h}=\widehat{g^{\prime}}=\widehat{k}$, uma contradição com o Lema 4.3.11.

Definimos em seguida a boa ordem que será utilizada para ordenar os conjuntos $H_{i}$ e mostrar que o algoritmo termina.

Seja $S$ um conjunto bem ordenado por uma ordem $<_{S}$. Então podemos definir uma boa ordem $\prec$ em $\operatorname{Fin}(S)$, da seguinte forma:

Sejam $A$ e $B$ em $\operatorname{Fin}(S)$. Então $A \preceq B$ se:

- $A=\emptyset$ ou

- $A \neq \emptyset$ e $\max (A)<_{S} \max (B)$ ou

- $A \neq \emptyset$ e $\max (A)=\max (B)$ e $(A \backslash\{\max (A)\}) \preceq(B \backslash\{\max (B)\})$.

Note o leitor que, uma vez que $A$ e $B$ são subconjuntos finitos de um conjunto bem ordenado, cada um possui um elemento máximo. Vejamos agora que a ordem $\prec$ definida acima é uma boa ordem.

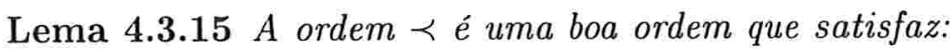

1. Se $A \subseteq B$, então $A \preceq B$.

2. Se $\max (A) \in B$, então $((B \cup A) \backslash\{\max (A)\}) \prec B$.

Demonstração. Vejamos primeiramente que se trata de uma boa ordem. Para tanto, é preciso mostrar que todo subconjunto $D$ de $\operatorname{Fin}(S)$ possui um menor elemento.

Antes de mais nada, notamos que, para todo $D \subseteq \operatorname{Fin}(S)$, o conjunto

$$
C_{D}:=\{\max (B) \mid B \in D\}
$$

é um subconjunto de $S$. Logo, $C_{D}$ possui um menor elemento, que chamaremos de $i(D)$. O restante da demonstração segue por redução ao absurdo (faremos uso da técnica de indução noetheriana, comentada na Seção 1.1). Assim, assumimos por absurdo que $\prec$ não é uma boa ordem. Logo, existe um subconjunto não vazio $N$ de $\mathcal{P}(\operatorname{Fin}(S))$ composto por todos os subconjuntos de $\operatorname{Fin}(S)$ que não possuem um menor elemento. Mais precisamente,

$$
N:=\{D \subseteq \operatorname{Fin}(S) \mid D \text { não possui um menor elemento }\}
$$

Mas, pelos comentários anteriores, $\{i(D) \mid D \in N\}$ é um subconjunto de $S$ e, portanto, possui um menor elemento. Com isso, podemos definir: 


$$
m:=\min (\{i(D) \mid D \in N\})
$$

Em outras palavras, se $D \subseteq \operatorname{Fin}(S)$ é tal que $i(D)<m$, então $D$ possui um menor elemento.

Tomamos agora um subconjunto $X \subseteq \operatorname{Fin}(S)$ pertencente a $N$. Por hipótese, $X$ não possui um menor elemento. Denotemos então

$$
i(X)=c \text { e } X_{c}:=\{A \in X \mid \max (A)=c\}
$$

Claro que, pela definição de $\prec$ todos os elementos de $X \backslash X_{c}$ são estritamente maiores que os elementos de $X_{c}$, de modo que, se $X_{c}$ possuir um menor elemento, esse certamente será o menor elemento de $X$. Desse modo, se $X_{c}$ for unitário, $X$ possui um menor elemento e chegamos a uma contradição com a suposição de $N$ ser não vazio.

Caso contrário, associe a cada $A$ em $X_{c}$ o conjunto $A^{-}$, definido como

$$
A^{-}:=A \backslash\{\max (A)\}
$$

Denote por

$$
X_{c}^{-}:=\left\{A^{-} \mid A \in X_{c}\right\}
$$

Mas então, $i\left(X_{c}^{-}\right)<i(X)$. Logo, por hipótese de indução, existe um conjunto distinguido $A^{-}$em $X_{c}^{-}$de forma que $A^{-}$é o menor elemento de $X_{c}^{-}$, segundo $\prec$. Portanto, é claro pela definição de $\prec$ que $A$ é o menor elemento de $X$. Logo, temos uma contradição com a hipótese de $N$ ser não vazio e concluímos que $\prec$ é uma boa ordem.

Vejamos agora as propriedades 1 e 2 da ordem $\prec$.

Para a primeira propriedade, note que se $A \subseteq B$, então, $\max (A) \leq \max (B)$. A prova segue então por indução em $\operatorname{card}(A)$. Com efeito, se $\operatorname{card}(A)=0, A \preceq B$ por definição. Assuma agora que $\operatorname{card}(A)>0$. Mas então, se $\max (A)<\max (B)$, segue que $A \prec B$. Senão, consideramos $A \backslash\{\max (A)\}$ e $B \backslash\{\max (B)\}$. Note que $(A \backslash\{\max (A)\}) \subseteq$ $(B \backslash\{\max (B)\})$ e o resultado segue da hipótese de indução.

A segunda parte pode ser vista pelo seguinte. Denote por $C:=((B \cup A) \backslash\{\max (A)\})$. Se chamamos de $b_{1}, b_{2}, \ldots, b_{n}$ os elementos de $B$ estritamente maiores que $\max (A)$, veremos que eles pertencem a $B$ e a $C$. Por outro lado, $\max \left(B \backslash\left\{b_{1}, b_{2}, \ldots b_{n}\right\}\right)=\max (A)$, mas $\max \left(\left(C \backslash\left\{b_{1}, b_{2}, \ldots b_{n}\right\}\right)\right)<_{S} \max (A)$, ou $\left(C \backslash\left\{b_{1}, b_{2}, \ldots b_{n}\right\}\right)=\emptyset$. O resultado segue então da definição de $\prec$.

A segunda propriedade do lema acima será particularmente interessante adiante e vale comentar melhor seu significado. Ela pode ser interpretada da seguinte forma: se substituirmos um elemento de um conjunto $B$ por elementos menores, o conjunto resultante será menor que $B$. O leitor é convidado a verificar que algo desse tipo ocorre com o suporte de um elemento quando esse sofre uma redução simples. 
Vejamos agora como podemos utilizar a ordem $\prec$ para mostrar que o algoritmo TLREDUz termina sua computação. Primeiro, podemos utilizar $\prec$ para dar uma boa ordem em $\operatorname{Fin}(\mathcal{P})$. Com isso, o conjunto de todos os suportes de elementos de $K \Gamma$ está bem ordenado. Mas então, como $\operatorname{Fin}(\mathcal{P})$ está bem ordenado, podemos utilizar a ordem $\prec$ novamente para bem-ordenar $\operatorname{Fin}(\operatorname{Fin}(\mathcal{P}))$. Assim, o conjunto composto por todos os conjuntos finitos de suportes de elementos de $K \Gamma$ está bem ordenado.

Diante do Lema 4.3.14, se identificarmos cada elemento de $H_{j}$ com seu suporte (para $j \geq 0$ ) teremos uma injeção. Assim, a boa ordem estabelecida em $\operatorname{Fin}(\operatorname{Fin}(\mathcal{P}))$ induz uma boa ordem em $\left\{H_{j}\right\}_{j \geq 0}$. Além disso, temos a seguinte afirmação.

Lema 4.3.16 A cada execução da linha 6 do algoritmo TLREDUZ, $\operatorname{supp}(k) \prec \operatorname{supp}\left(f^{\prime}\right)$. Conseqüentemente, $H_{j} \prec H_{j-1}$, para todo $j \geq 1$.

Demonstração. Se $k=0$, então $\operatorname{supp}(k)=\emptyset$, e o resultado segue. Se $k \neq 0$, então, pelo Lema 4.3.11, $\max (\operatorname{supp}(k))<\max \left(\operatorname{supp}\left(f^{\prime}\right)\right)$ e segue o resultado.

A segunda parte segue imediatamente do Lema 4.3.15.

Com isso, podemos apresentar a Proposição 4.3.17, que afirma a correção do algoritmo TLREDUZ.

Proposição 4.3.17 O algoritmo TLREDUZ termina sua computação em um número finito de passos. Ainda, se o algoritmo recebe como entrada um conjunto $H$, ele produz como saída um conjunto $H^{\prime}$, satisfazendo:

1. O ideal gerado por $H^{\prime}$ é igual ao ideal gerado por $H$.

2. O conjunto $H^{\prime}$ é finito e tl-reduzido.

3. O conjunto $H^{\prime}$ é uniforme.

Demonstração. $O$ fato de que o algoritmo termina sua computação é conseqüência imediata da boa ordem $\prec$ definida em $\left\{H_{j}\right\}_{j \geq 0}$ e do Lema 4.3.16.

A propriedade 1 da saída do algoritmo segue imediatamente do Corolário 4.1.3. A propriedade 2 é de verificação imediata. Finalmente, a propriedade 3 segue do Lema 4.2 .8 .

Podemos utilizar a Proposição 4.2 .5 e os resultados que obtivemos até aqui para destacar a seguinte propriedade dos ideais finitamente gerados de $K \Gamma$.

Proposição 4.3.18 Todo ideal finitamente gerado de $K \Gamma$ possui um conjunto gerador finito, tlreduzido e uniforme.

Tendo estabelecido a correção do algoritmo TLREDLZ, nos concentramos em algumas propriedades adicionais de sua saída que nos serão úteis nas demonstrações de correção de nossa segunda versão do Procedimento de Mora.

Para estes resultados, denotaremos por $H$ um conjunto fornecido como entrada para o algoritmo TLREDUz (satisfazendo as hipóteses do algoritmo sobre a entrada) e por $H^{\prime}$ a saída produzida por ele. 
Lema 4.3.19 Seja $g$ um elemento de $H$. Então, para todo $h$ em $H^{\prime} \backslash H, \widehat{h} \neq \widehat{g}$.

Demonstração. Suponha que o lema seja falso e tome $h$ e $g$ nas condições do enunciado com $\widehat{h}=\widehat{g}$. Logo, como $h \notin H$, existe $j>0$ tal que $h \notin H_{j-1}$, mas $h \in H_{j}$, isto é, $h$ é inserido em $H_{j}$ na $j$-ésima iteração.

Por outro lado, pelo Lema 4.3.12, existe $g^{\prime}$ em $H_{j-1}$ tal que $\widehat{g^{\prime}} \mid \widehat{g}$. Mas então $\widehat{g^{\prime}} \mid \widehat{h}$ e temos uma contradição com a Afirmação 4.3 .9 , ou $g^{\prime}$ é o valor da variável $f^{\prime}$ durante essa iteração. Mas nesse segundo caso, concluímos que $\widehat{h}<\widehat{g^{\prime}}$ pelo Lema 4.3 .11 e chegamos a uma contradição com o fato de que $\widehat{g^{\prime}} \mid \widehat{g}=\widehat{h}$.

Lema 4.3.20 Se s é um S-elemento que possui representação de Gröbner em termos de H, então s possui representação de Gröbner em termos de $H^{\prime}$.

Demonstração. Seja $s$ um S-elemento da forma $s=l g_{i} r-\alpha l^{\prime} g_{j} r^{\prime}$ que possui representação de Gröbner em termos de $H$. Então,

$$
s=\sum_{k, u} \alpha_{k, u} l_{k, u} g_{k} r_{k, u}
$$

com $\alpha_{k, u}$ escalares, $g_{k}$ em $H$ e $l_{k, u}$ e $r_{k, u}$ em $\mathcal{P}$, satisfazendo $l_{k, u} \widehat{g_{k}} r_{k, u}<l \widehat{g}_{i} r=l^{\prime} \widehat{g}_{j} r^{\prime}$ Mostraremos que o algoritmo possui o seguinte invariante: no início de cada execução da linha 3 do algoritmo TLReduz, $s$ possui uma representação de Gröbner em termos de $H_{i}$. Claro que o invariante é válido no início da primeira iteração, dado que $H_{0}=H$.

Suponha agora o invariante válido no início da $j$-ésima execução da linha 3 (e que não se trata da última execução dessa linha) e vejamos que ele se mantém válido no início da $(j+1)$-ésima execução dessa mesma linha. Mas durante a iteração, algum elemento $h$ de $H_{i}$ é substituído por $k$ (ou simplesmente removido, se $k$ for nulo). Com isso, pela Proposição 4.1.2, teremos:

$$
h=\sum_{l, v} \alpha_{l, v} b_{l, v} f_{l} b_{l, v}^{\prime}+h^{\prime}
$$

com $\alpha_{l, v}$ escalares, $b_{l . v}$ e $b_{l . v}^{\prime}$ monômios, $f_{l}$ em $H_{i}$ e $h^{\prime}=k$, satisfazendo $b_{l, v} \widehat{f}_{l} b_{l, v}^{\prime} \leq \widehat{h}$ e $\widehat{h^{\prime}} \leq \widehat{h}$.

Com isso, se $h$ não aparece na Equação $4.3, s$ possui uma representação de Gröbner em termos de $H_{i}$ na $(j+1)$-ésima iteração trivialmente. Por outro lado, se $h$ aparece na Equação 4.3, basta substituí-lo pela Equação 4.4 e utilizar a Afirmação 4.3 .10 juntamente com o Lema 4.2.4 para obter uma representação de Gröbner para $s$ em termos de $H_{i}$.

Proposição 4.3.21 Para todo $\mathrm{g} \mathrm{em} H \backslash H^{\prime}$,

$$
g=\lambda u g_{i} v+\sum_{k, l} \alpha_{k \cdot l} u_{k, l} g_{k} \cdot v_{k, l}
$$


com $\lambda$ e $\alpha_{k, l}$ escalares, $u, v, u_{k, l}$ e $v_{k, l}$ monômios e $g_{i}$ e $g_{k}$ em $H^{\prime}$. Ainda,

$$
\widehat{g}=u \widehat{g_{i}} v e u_{k, l} \widehat{g_{k}} v_{k, l}<\widehat{g}
$$

Demonstração. Mostraremos que a propriedade acima é válida no início de cada execução da linha 3 do algoritmo, com $H_{i}$ no lugar de $H^{\prime}$.

Ela é válida no início da primeira execução de forma trivial, dado que $H \backslash H_{0}=\emptyset$. Suponha agora que ela seja válida no início da $j$-ésima execução da linha 3 (e que não se trata da última execução dessa linha) e vejamos que ela se mantém válida no início da $(j+1)$-ésima execução desta linha.

Mas durante a iteração, algum elemento $h$ de $H_{i}$ é substituído por $k$ (ou simplesmente removido, se $k$ for nulo). Com isso, pela Proposição 4.1.2, teremos:

$$
h=\sum_{l, v} \alpha_{l, v} b_{l, v} f_{l} b_{l, v}^{\prime}+h^{\prime}
$$

com $\alpha_{l, v}$ escalares, $b_{l, v}$ e $b_{l, v}^{\prime}$ monômios, $f_{l}$ em $H_{i}$ e $h^{\prime}=k$, satisfazendo $b_{l, v} \widehat{f}_{l} b_{l, v}^{\prime} \leq \widehat{h}$ e $\widehat{h^{\prime}} \leq \widehat{h}\left(\right.$ se $\left.h^{\prime} \neq 0\right)$.

Considere então a $(j+1)$-ésima execução da linha 3 e tome $g$ em $H \backslash H_{i}$ neste momento. Temos duas possibilidades:

- O elemento $g$ não pertence a $H \backslash H_{i-1}$.

Neste caso, $g \in H_{i-1}$, e concluímos que $g=h$ pelo próprio funcionamento do algoritmo TLREduz. O resultado segue então da Equação 4.5, da Proposição 4.1.2 e do Lema 4.2.4.

- O elemento $g$ pertence a $H \backslash H_{i-1}$.

Neste caso, $g$ se escreve na soma do enunciado por hipótese de indução. Logo, por um lado, se $h$ não ocorre nessa soma, o invariante se mantém válido trivialmente. Por outro lado, se $h$ ocorre nesta soma, basta substituir a Equação 4.5 na soma e a propriedade se manterá válida pela Proposição 4.1 .2 e pelo Lema 4.2.4.

\subsubsection{Uma Segunda Versão}

Apresentamos aqui uma segunda versão do Procedimento de Mora. Esta versão faz uso do algoritmo TLREDUZ (Algoritmo 4.3.3) apresentado anteriormente e possui a propriedade adicional de produzir uma Base de Gröbner minimal (caso termine sua computação). Segue a descrição do procedimento no Algoritmo 4.3.4.

Comentamos que em vista dos algoritmos PrePara (Algoritmo 4.3.2) e TLREduz (Algoritmo 4.3.3) vistos na seção anterior, fica claro que a hipótese feita pelo procedimento de que o conjunto $F$ seja tl-reduzido não constitui nenhuma perda de generalidade.

O procedimento faz uso de algumas funções auxiliares já vistas para a primeira versão. Ele também utiliza a função ATLALIZASOBREPOSIÇÕES, que descrevemos brevemente a seguir. 


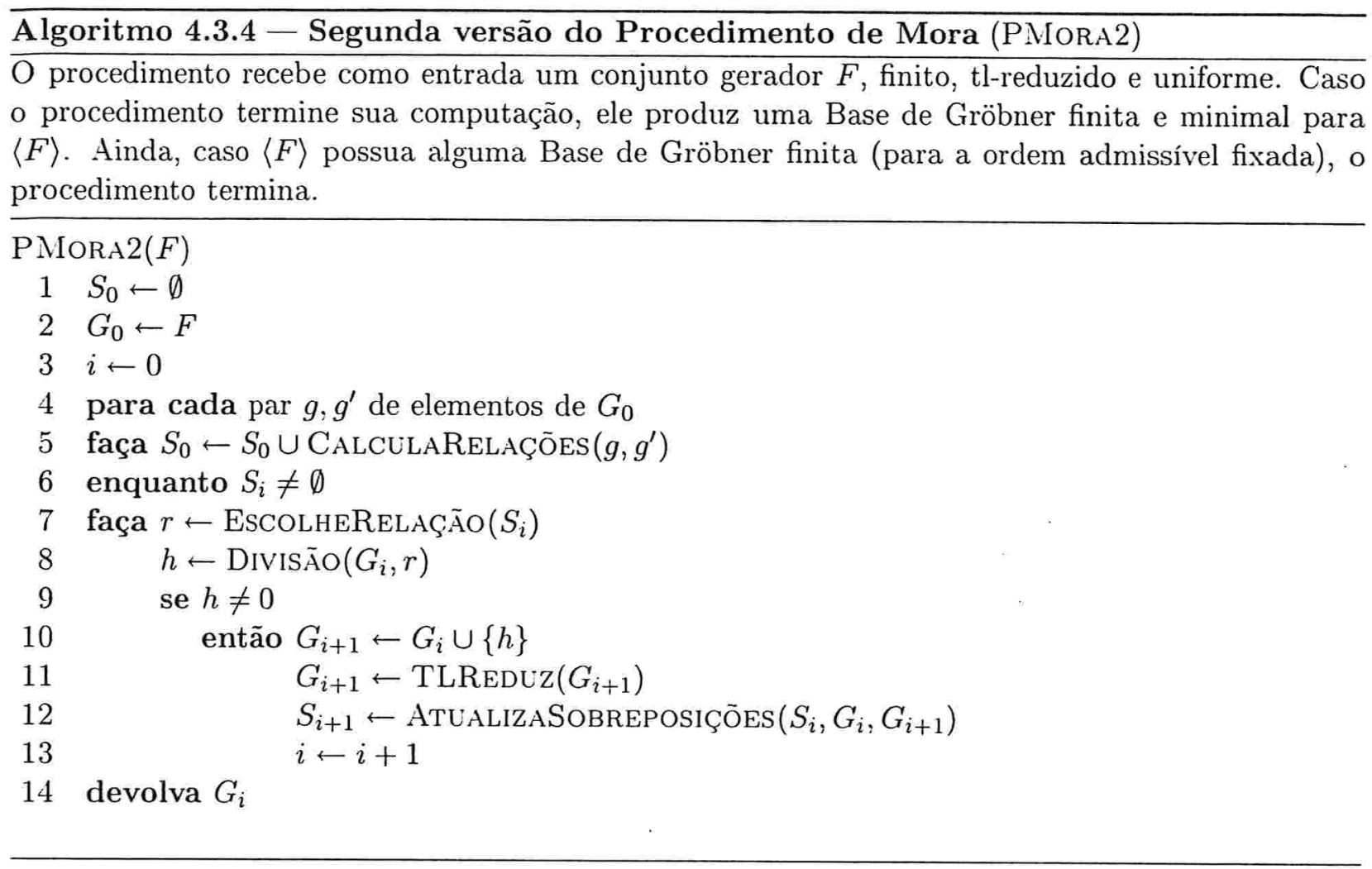

AtualizaSobreposições $\left(S, G, G^{\prime}\right)$ Esta função retorna um conjunto de relações de sobreposição não nulas entre elementos de $G^{\prime}$. Ela encontra:

- As relações de sobreposição não nulas em $S$ que envolvem elementos $g$ e $g^{\prime}$, ambos pertencentes a $G^{\prime}$.

- As relações de sobreposição não nulas que envolvem elementos $g$ e $g^{\prime}$ de forma que ambos pertencem a $G^{\prime}$, mas somente um a $G$.

- As relações de sobreposição não nulas envolvendo dois elementos de $G^{\prime} \backslash G$.

Em outras palavras, a função ATUALiZASOBREPosições devolve as relações de sobreposição não nulas envolvendo elementos de $G^{\prime}$ que ainda não foram consideradas pelo procedimento.

A seguir, revisitamos o Exemplo 4.3 .1 com o intuito de ilustrar o funcionamento dessa segunda versão do Procedimento de Mora. ${ }^{22}$

Exemplo 4.3.4 Considere a álgebra $K \Gamma$ e o conjunto $F$ vistos no Exemplo 4.3 .1 e fixe a mesma ordem em $\mathcal{P}$ vista naquele exemplo. Note que o conjunto $F$ é tl-reduzido e uniforme. ${ }^{23}$ Vejamos então uma simulaşão de uma chamada para P\IorA2 $(F)$.

\footnotetext{
${ }^{2}$ A luz do que fizemos nos Exemplos 4.3.1 e 4.3.2, consideraremos os conjuntos $S_{2}$ implementados como uma única fila para as simulaçōes.

${ }^{23}$ Caso $F$ nāo fosse um conjunto tl-reduzido, as funçōes Prepara e TLReduz poderiam ser utilizadas para obter um conjunto gerador tl-reduzido e uniforme para o mesmo ideal.
} 
Como antes, inicialmente $G_{0} \leftarrow F$ e o laço das linhas 4 e 5 calcula as seguintes relações de sobreposição entre os elementos de $G_{0}$ :

$$
\begin{aligned}
& s_{1}=O\left(g_{2}, g_{1}, c d a, c\right)=c d a g_{2}-g_{1} c=-c d a d a+c b c \\
& s_{2}=O\left(g_{1}, g_{2}, b, d a b\right)=b g_{1}-g_{2} d a b=d a d a b-b c b \\
& s_{3}=O\left(g_{3}, g_{2}, b, \text { dadab }\right)=b g_{3}-g_{2} d a d a b=(d a)^{3} b-b c b \\
& s_{4}=O\left(g_{2}, g_{3}, c \text { dada, } c\right)=c \text { dadag } g_{2}-g_{3} c=-c(d a)^{3}+c b c
\end{aligned}
$$

Com isso, imediatamente antes da primeira execução da linha 6, teremos:

$$
\begin{aligned}
& G_{0}=\left\{g_{1}, g_{2}, g_{3}\right\} \\
& S_{0}=\left(s_{1}, s_{2}, s_{3}, s_{4}\right)
\end{aligned}
$$

O procedimento considera então $s_{1}$ :

$$
s_{1} \Rightarrow_{G_{0}}-c d a d a+c d a:=g_{4}
$$

Assim, na linha 10:

$$
G_{1} \leftarrow G_{0} \cup\left\{g_{4}\right\}=\left\{g_{1}, g_{2}, g_{3}, g_{4}\right\}
$$

E na linha 11, teremos:

$$
G_{1} \leftarrow \operatorname{TLReduz}\left(G_{1}\right)
$$

Mas, como imediatamente após o términos da linha $10, G_{1}$ não é tl-reduzido, ele será modificado pelo algoritmo TLREDUz e ao final da linha 11 obteremos:

$$
G_{1}=\left\{g_{1}, g_{2}, g_{4}\right\}
$$

Ainda, a chamada para a função AtUalizaSobreposições $\left(S_{0}\right)$ da linha 12 nos dará:

$$
S_{1} \leftarrow\left\{s_{2}\right\}
$$

Com isso, a primeira iteração é terminada e o procedimento considera $s_{2}$ :

$$
s_{2} \Rightarrow_{G_{1}} d a d a b-d a b:=g_{5}
$$

Assim, $G_{2} \leftarrow\left\{g_{1}, g_{2}, g_{4}, g_{5}\right\}$ e, como é um conjunto tl-reduzido, $G_{2}$ não será modificado pela chamada TLRediz $\left(G_{2}\right)$ da linha 11 .

Porém, existem relações de sobreposição entre $g_{5}$ e elementos de $G_{2}$. Estas são calculadas pela função ATUALıZASOBREPOSIÇŌES:

$$
\begin{aligned}
& s_{5}=O\left(g_{2}, g_{5}, d a d a, c\right)=\operatorname{dadag}_{2}-g_{5} c=-(d a)^{3}+d a b c \\
& s_{6}=O\left(g_{5}, g_{4}, c, b\right)=c g_{5}+g_{4} b=0
\end{aligned}
$$


Com isso, $S_{2} \leftarrow\left(s_{5}\right)$.

Logo, o procedimento considera $s_{5}$ e teremos:

$$
s_{5} \Rightarrow_{g_{2}}-(d a)^{3}+d a d a:=g_{6}
$$

Assim, na linha $10, G_{3} \leftarrow\left\{g_{1}, g_{2}, g_{4}, g_{5}, g_{6}\right\}$. Novamente, como $G_{3}$ é tl-reduzido neste ponto, ele não será modificado pela chamada a TLREDUZ na linha 11.

Ainda, a função ATUALIZASobreposições computa as seguintes relações de sobreposição entre $g_{6}$ e elementos de $G_{3}$ :

$$
\begin{aligned}
& s_{7}=O\left(g_{6}, g_{4}, c, d a\right)=c g_{6}-g_{4} d a=0 \\
& s_{8}=O\left(g_{5}, g_{6}, d a, b\right)=d a g_{5}+g_{6} b=0 \\
& s_{9}=O\left(g_{6}, g_{6}, d a, d a\right)=d a g_{6}-g_{6} d a=0 \\
& s_{10}=O\left(g_{6}, g_{6},(d a)^{2},(d a)^{2}\right)=(d a)^{2} g_{6}-g_{6}(d a)^{2}=0
\end{aligned}
$$

Logo, $S_{3} \leftarrow \emptyset$ e o procedimento termina, devolvendo:

$$
G_{3}=\left\{g_{1}, g_{2}, g_{4}, g_{5}, g_{6}\right\}
$$

$\Delta$

O leitor interessado é convidado a verificar que essa segunda versão do procedimento também não termina sua computação ao receber como entrada o conjunto gerador do Exemplo 4.3.2; com efeito ele produz exatamente a mesma saída.

Ainda, é importante ressaltar que, tal qual exigido para a primeira versão, também para esta é necessário que as relações de sobreposição inseridas nos conjuntos $S_{i}$ sejam consideradas em tempo finito pelo procedimento. Convidamos o leitor cético a utilizar a segunda versão do procedimento em conjunto com o Exemplo 4.3.3 e observar o mesmo fenômeno visto naquele caso.

Comentamos que, apesar de o procedimento PMORA2 devolver uma Base de Gröbner minimal para o ideal em questão, ela não será necessariamente reduzida. Esse fato é mostrado no exemplo abaixo. A Seção 4.3.4 cuidará de apresentar um procedimento que encontra a Base de Gröbner reduzida de um ideal (caso ela seja finita).

Exemplo 4.3.5 Considere o contexto do Exemplo 3.2.1. Então, ao fornecermos qualquer conjunto $G_{i}$ para o procedimento PMora2, em que $i \in \mathbb{N}^{*}$, teremos como saída precisamente o conjunto $G_{i}$ fornecido como entrada.

Por outro lado, não é difícil ver que $a^{3}-b^{2 i}$ não é reduzido, para todo $i \in \mathbb{N}^{*}\left(b^{2 i}\right.$ é divisivel por $b^{2}$ e, portanto, pertence a $\left.\langle\widehat{I}\rangle\right)$.

Voltamo-nos em seguida para demonstrar a correção do procedimento PMoRA2. Começamos com a observação análoga à feita para a primeira versão pelo Lema 4.3.2. Afirmamos então que o laço das linhas 4 a 5 termina sua computação. Esta afirmação segue, como antes, da finitude do conjunto $G_{0}=F$ e da efetividade da função CALClLARELAÇões.

Lema 4.3.22 Imediatamente antes de cada chamada de TLREDUz na linha 11, $G_{i+1}$ é tal que os suportes de seus elementos säo dois a dois distintos. 
Demonstração. Note que no início da primeira execução da linha $10, G_{0}$ é um conjunto tl-reduzido e, portanto, os suportes de seus elementos são dois a dois distintos. Por outro lado, afirmamos que o suporte de $h$ é diferente do suporte de cada elemento de $G_{1}$ (distinto de $h$ ). Com efeito, suponha que isso não é verdade. Então, existe $g \neq h$ em $G_{1}$, tal que $\operatorname{supp}(g)=\operatorname{supp}(h)$. Logo, $\widehat{g}=\widehat{h}$, nos levando a uma contradição com a Proposição 4.1.2. Portanto, concluímos que os suportes dos elementos de $G_{1}$ são dois a dois distintos no início da primeira execução da linha 11 . Logo, o conjunto $G_{1}$ será tl-reduzido, após a atribuição da linha 11.

O resultado segue então por um argumento indutivo.

Lema 4.3.23 No início de cada execução da linha 6 do procedimento PMORA2, os seguintes invariantes são válidos:

1. O conjunto $S_{i}$ contém todas as relações de sobreposição envolvendo elementos de $G_{i}$ que não possuem representação de Gröbner em termos de $G_{i}$.

2. Os elementos de $G_{i}$ são todos uniformes.

3. $O$ conjunto $G_{i}$ é tl-reduzido e finito.

4. $O$ ideal gerado por $G_{i}$ é igual ao ideal gerado por $F$.

5. O conjunto $S_{i}$ é finito e composto somente por relações de sobreposição envolvendo elementos de $G_{i}$.

Demonstração. As propriedades 2 e 4 seguem imediatamente do Lema 4.2.8, do Corolário 4.1.3 e da Proposição 4.3.17. A propriedade 5 segue da Proposição 4.2 .29 e das propriedades da função AtualizaSobreposições. A propriedade 3, por sua vez, é de verificação imediata.

Vejamos em seguida a propriedade 1 . O laço das linhas 3 e 4 nos garante que ela será trivialmente verdadeira no início da primeira execução da linha 6 . Suponha agora que ela seja válida no início da $j$-ésima execução dessa linha (e que não se trata da última execução dessa linha) e vejamos que ela se mantém válida no início da $(j+1)$-ésima execução da linha 6.

Mas, durante o laço das linhas 6 a 13, ou uma relação que se reduz para zero sobre $G_{i}$ é removida de $S_{i}$ e o invariante se mantém válido, ou a condição da linha 9 é falsa. Suponha então que ocorre o segundo caso e considere o início da $(j+1)$-ésima execução da linha 6. Mas então, a função AtualizASobreposições cuidou de inserir em $S_{i}$ todas as relações de sobreposição envolvendo elementos de $G_{i} \backslash G_{i-1}$.

Basta, portanto verificar que se uma relação de sobreposição $s$ envolvendo elementos de $G_{i} \cap G_{i-1}$ não pertence a $S_{i}$, então ela possui uma representação de Gröbner em termos de $G_{i}$. Mas, por hipótese de indução, temos duas possibilidades:

- A relação $s$ pertence a $S_{i-1}$, donde concluímos que $s$ pertence a $S_{i}$ pelo funcionamento da função AtralizaSobreposições. 
- A relação $s$ não pertence a $S_{i-1}$, donde concluímos (por hipótese de indução) que $s$ possui representação de Gröbner em termos de $G_{i-1}$. O resultado segue então do Lema 4.3.20.

Lema 4.3.24 O procedimento PMORA2 termina sua computação se e somente se $G_{j}$ é uma Base de Gröbner finita e minimal para $\langle F\rangle$, para algum $j$.

Demonstração. Se o procedimento pára, o resultado segue dos invariantes do Lema 4.3.23 e da Proposição 4.2.30 (note o leitor que, como $G_{j}$ é tl-reduzido, não existem relações de divisão envolvendo elementos de $G_{j}$ ).

Por outro lado, se $G_{j}$ é uma Base de Gröbner, para algum $j$, então segue do Teorema 4.2.31 que todas as relações de sobreposição envolvendo elementos de $G_{j}$ se reduzem para zero sobre $G_{j}$. Assim, como o invariante 5 do Lema 4.3.23 nos garante que todas as relações em $S_{j}$ são relações entre elementos de $G_{j}$, o procedimento terminará sua computação.

Claro que $G_{j}$ é uma Base de Gröbner minimal, pelo fato de ser tl-reduzido e pelo Lema 3.2 .8 .

Voltamo-nos agora para mostrar que se $\langle F\rangle$ possui alguma Base de Gröbner finita, então PMora2 $(F)$ termina sua computação.

Lema 4.3.25 A seguinte afirmação é válida no início de cada execução da linha 6: se g pertence a $G_{l}$, para algum $l \geq 0$ e existe $j>l$ tal que $g$ não pertence $a G_{j}$, então:

$$
g=\sum_{h, k} \alpha_{h, k} u_{h, k} g_{h} v_{h, k}
$$

com $\alpha_{h, k}$ escalares, $u_{h, k}$ e $v_{h, k}$ monômios e $g_{h}$ em $G_{j}$, para todos $h, k$. Ainda, $\widehat{g_{h}}<\widehat{g}$ e $u_{h, k} \widehat{g_{h}} v_{h, k} \leq$ $\widehat{g}$, para todos $h, k$.

Demonstração. Tome $g$ nas condições do enunciado. Então existe um inteiro $z$ tal que $g \in G_{z}$, mas $g \notin G_{z+1}$. Pelo funcionamento de PMorA2, é evidente que $g \in G_{z+1}$ ao final da execução da linha 10 e ele foi removido durante a chamada TLREduz $\left(G_{z+1}\right)$. Logo, pela Proposição 4.3.21, temos que:

$$
g=\lambda u g_{p} v+\sum_{h, k} \alpha_{h, k} u_{h, k} g_{h} v_{h, k}
$$

$\operatorname{com} \lambda, \alpha_{h, k}$ escalares, $u, v, u_{h . k}$ e $v_{h, k}$ monômios e $g_{h}, g_{p}$ em $G_{z+1}$, para todos $h, k$. Ainda, $\widehat{g}=u \widehat{g_{p}} v, \widehat{g_{h}}<\widehat{g}$ e $u_{h . k} \widehat{g_{h}} v_{h, k} \leq \widehat{g}$, para todos $h, k$.

Portanto, resta-nos mostrar que $\widehat{g_{p}}<\widehat{g}$. Claro que basta mostrar que $\widehat{g_{p}} \neq \widehat{g}$.

Mas, pelo fato de $G_{z}$ ser tl-reduzido, concluímos que $g_{p} \notin G_{z}$. Portanto, há duas possibilidades: 
1. Durante a iteração em questão, $g_{p}=h$.

Mas então, como $h$ é o resultado de uma redução sobre $G_{z}$, segue da Proposição 4.1.2 que $\widehat{g_{p}} \neq \widehat{g}$.

2. O elemento $g_{p}$ foi inserido em $G_{z+1}$ durante a execução de $\operatorname{TLReduz}\left(G_{z+1}\right)$.

Neste caso, $\widehat{g_{p}} \neq \widehat{g}$ pelo Lema 4.3.19.

O resultado segue então por um argumento indutivo.

A luz do que foi feito para a primeira versão, convencionamos que se o procedimento pára com $i=k$, para algum $k$, então $G_{j}:=G_{k}$, para todo $j>k$. Com isso, definimos como antes o conjunto $G_{\infty}$ :

$$
G_{\infty}:=\bigcup_{j=0}^{\infty} G_{j}
$$

Definimos ainda, para cada $j$ :

$$
\overline{G_{j}}:=\left\{g \in G_{j} \mid g \in G_{v} \text { para todo } v \geq j\right\}
$$

$\mathrm{e}$

$$
\overline{G_{\infty}}:=\bigcup_{j=0}^{\infty} \overline{G_{j}}
$$

A seguinte afirmação é conseqüência imediata da definição de $\overline{G_{j}}$. Destacamo-na abaixo para futura referência.

Afirmação 4.3.26 Se $k \geq j$, então $\overline{G_{j}} \subseteq \overline{G_{k}}$.

Proposição 4.3.27 Se g pertence a $G_{\infty}$, então

$$
g=\sum_{h, k} \alpha_{h, k} u_{h, k} g_{h} v_{h, k}
$$

com $\alpha_{h, k}$ escalares, $u_{h, k}$ e $v_{h, k}$ monômios e $g_{h}$ em $\overline{G_{\infty}}$, para todos $h, k$. Ainda, $\widehat{g_{h}} \leq \widehat{g}$, para todo $h$.

Demonstração. Suponha por absurdo que o resultado seja falso e tome $f$ um contra exemplo de termo líder mínimo. Claro que $f \notin \overline{G_{\infty}}$. Portanto, existem $j$ e $l$, com $j<l$, tais que $f \in G_{j}$, mas $f \notin G_{l}$. Logo, pelo Lema 4.3.25, $f$ se escreve como uma soma na forma do enunciado (exceto pelo fato de que $g_{h}$ pertence a $G_{l}$, mas não necessariamente a $\left.\overline{G_{\infty}}\right)$, satisfazendo $\widehat{g_{h}}<\widehat{f}$, para todo $h$.

Assim, pela minimalidade de $\widehat{f}$, cada um dos elementos $g_{h}$ se escreve como uma soma na forma do enunciado, donde segue o resultado.

Como conseqüència da proposição anterior, chegamos aos seguintes corolários.

Corolário 4.3.28 O ideal gerado por $F$ é igual ao ideal gerado por $\overline{G_{x}}$. 


\section{Corolário 4.3.29 O conjunto $\overline{G_{\infty}}$ é uma Base de Gröbner.}

Demonstração. Afirmamos primeiramente que $\overline{G_{\infty}}$ é tl-reduzido. Com efeito, tome $g, g^{\prime}$ em $\overline{G_{\infty}}$. Então, pela definição de $\overline{G_{\infty}}$, existem $j, k$ tais que $g \in \overline{G_{j}}$ e $g^{\prime} \in \overline{G_{k}}$. Ainda, podemos assumir, sem perda de generalidade, que $j \leq k$. Mas então, pela Afirmação 4.3.26, $\overline{G_{j}} \subseteq \overline{G_{k}} \subseteq G_{k}$. Assim, como $G_{k}$ é tl-reduzido (pelo Lema 4.3.23), segue que $\widehat{g} \nmid \widehat{g^{\prime}}$ e $\widehat{g^{\prime}} \nmid \widehat{g}$.

Note ainda que o Lema 4.3.23 nos garante que os elementos de $\overline{G_{\infty}}$ são todos uniformes.

Considere agora uma relação de sobreposição $s$ envolvendo elementos $g$ e $g^{\prime}$ de $\overline{G_{\infty}}$. Mas, como $g$ e $g^{\prime}$ pertencem a $\overline{G_{\infty}}$, s nunca é removido pela função ATUALIZASOBREPOSIÇões. Logo, ele será considerado em algum tempo finito pela função EscolheRELAÇĀo e, portanto, se reduz para zero sobre $G_{\infty}$. Podemos então utilizar a Afirmação 4.2 .25 para concluir que $s$ possui uma representação de Gröbner em termos de $G_{\infty}$. Portanto, pela Proposição 4.3.27, $s$ possui uma representação de Gröbner em termos de $\overline{G_{\infty}}$.

O resultado segue então da Proposição 4.2.30.

Corolário 4.3.30 O conjunto $G_{\infty}$ é uma Base de Gröbner.

Demonstração. Segue imediatamente do fato de que $\overline{G_{\infty}} \subseteq G_{\infty}$.

Finalmente podemos resumir o que foi discutido até aqui no seguinte teorema.

Teorema 4.3.31 Seja $F$ um subconjunto finito, uniforme e tl-reduzido de KГ. Então $\langle F\rangle$ possui uma Base de Gröbner finita se e somente se PMORA2 $(F)$ termina sua computação, devolvendo uma Base de Gröbner finita e minimal para $\langle F\rangle$.

Demonstração. Para verificar a validade do teorema, basta utilizar o Lema 4.3 .24 e um argumento completamente análogo ao visto na demonstração do Teorema 4.3.7.

\subsubsection{Bases de Gröbner Reduzidas}

Nesta seção comentamos brevemente como as ferramentas desenvolvidas até aqui podem ser utilizadas para encontrar a Base de Gröbner reduzida para um ideal de $K \Gamma$ a partir de um conjunto gerador finito (caso ele admita alguma Base de Gröbner finita).

Considere então $F$ um conjunto gerador finito para um ideal $I$ de $K \Gamma$, de modo que $F$ seja tl-reduzido e composto somente por elementos uniformes. ${ }^{24}$

Utilizando o Teorema 4.3.31 e o Corolário 3.2.15 podemos ver que PMora2 $(F)$ termina sua computação se e somente se a Base de Gröbner reduzida para $I$ é finita.

Assim, dada uma Base de Gröbner minimal finita $G$ para $I$, o Corolário 3.2.14 afirma que basta considerar cada elemento de $\widehat{G}$ e encontrar sua forma normal.

Podemos resumir a discussão acima no procedimento BGREDUZIDA (Algoritmo 4.3.j). É claro que ele termina sua computação se e somente se $I=\langle F\rangle$ possui uma Base de Gröbner reduzida finita.

\footnotetext{
${ }^{24}$ Como já comentado na Seção 4.3.3, essas exigências sobre $F$ não constituem nenhuma perda de generalidade.
} 


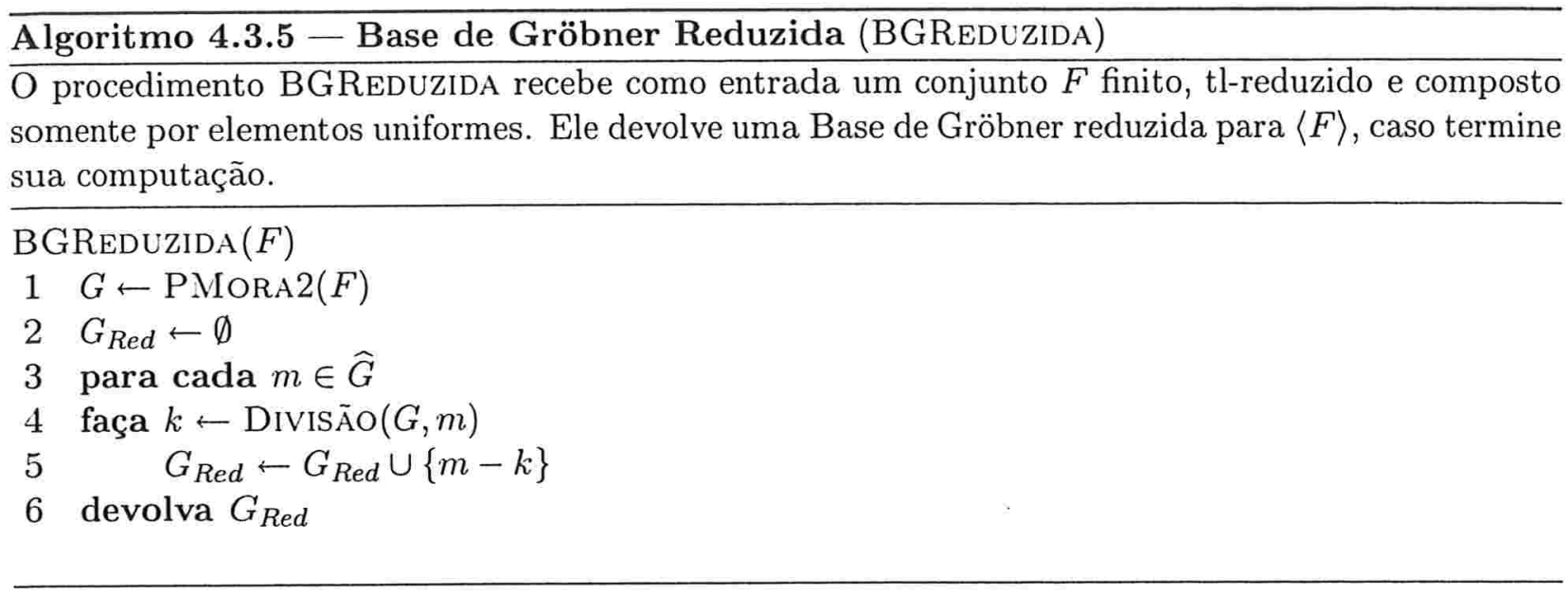

Para ilustrar o funcionamento do procedimento acima, convidamos o leitor a revisitar o Exemplo 4.3.4 e verificar que a saída obtida naquele exemplo é a mesma obtida ao se fornecer o conjunto $F$ definido no exemplo para o procedimento BGREDuzIDA. Isso mostra que a Base de Gröbner calculada pelo procedimento PMora2 naquele caso é reduzida.

Por outro lado, o mesmo não acontece ao considerar o Exemplo 4.3.5, como mostrado abaixo:

Exemplo 4.3.6 Considere o contexto do Exemplo 4.3.5 e suponha que um conjunto $G_{i}=\left\{a^{3}-b^{2 i}, b^{2}\right\}$ (para algum $i \in \mathbb{N}^{*}$ ) tenha sido fornecido como entrada para o procedimento BGREDUZIDA.

Como já comentado no Exemplo 4.3.5, a saída de PMora2 será exatamente o conjunto $G_{i}$, de forma que $G \leftarrow G_{i}$, na linha 1 de BGReduzida. Assim, vemos que:

$$
\begin{aligned}
& \widehat{G}=\left\{a^{3}, b^{2}\right\} \\
& a^{3} \Rightarrow_{G_{i}} 0 \\
& b^{2} \Rightarrow_{G_{i}} 0
\end{aligned}
$$

Portanto, a saída de BGReduzida $\left(G_{i}\right)$ será $G_{\text {Red }}=\left\{a^{3}, b^{2}\right\}$, claramente a Base de Gröbner reduzida para $I$.

\subsection{Computabilidade de Bases de Gröbner}

Conforme visto na seção anterior, o Procedimento de Mora pode nunca terminar sua computação. Nosso objetivo nesta seção é explorar algumas questões de computabilidade relacionadas com a Teoria de Bases de Gröbner não comutativas. Como consequiência, veremos que o fato de o procedimento visto na Seção 4.3 não terminar sua computação não constitui uma fraqueza na estratégia empregada em seu desenvolvimento, mas sim uma restrição lógica da própria teoria.

Primeiramente, lembramos que o Exemplo 3.2.2 nos mostra que existem um alfabeto $X$, uma ordem admissivel $\leq$ e um ideal finitamente gerado $I$ de $K\langle X\rangle$, de modo que $I$ não admite nenhuma Base de Gröbner finita com respeito à base $X^{*}$ e à ordem $\leq$.

Assim, não há esperanças de encontrar um procedimento que, no contexto das álgebras de caminhos (com uma base multiplicativa e uma ordem admissivel arbitrárias) receba como entrada 
um conjunto finito de geradores de um ideal $I$ e devolva uma Base de Gröbner para $I$, listando seus elementos. ${ }^{25}$ Certamente, tal procedimento nunca terminaria sua computação. Por outro lado, o Procedimento de Mora é capaz de enumerar os elementos de uma Base de Gröbner para um ideal $I$, a partir de finitos geradores desse ideal.

Ademais, nosso interesse em construir uma Base de Gröbner $G$ está na possibilidade de utilizá-la em conjunto com o Algoritmo da Divisão para efetuar reduções. Com efeito, esse último necessita de uma maneira de encontrar um elemento $g$ de $G$, de modo que $\widehat{g}$ divida um dado monômio $m$ (ou ter a garantia de que tal elemento não existe).

Claro que no caso em que $G$ é finito, existe uma forma natural de se realizar tal operação. Entretanto, apesar de a descrição do Algoritmo da Divisão dada na Seção 4.1 (Algoritmo 4.1.1) exigir que o conjunto de entrada $S$ seja finito, não é difícil ver que essa finitude é exigida tão somente para que a operação descrita acima possa ser realizada.

Assim, uma próxima pergunta natural seria se é possível representar uma Base de Gröbner infinita por meio de uma estrutura finita e que seja capaz de responder à pergunta mencionada. Mais precisamente, queremos uma representação finita $E$ que represente uma Base de Gröbner (possivelmente infinita) $G$ e um algoritmo $\mathcal{D}$, que recebe com parâmetros de entrada $E$ e um monômio $m$ e fornece como saída ou um elemento $g$ de $G$ de modo que $\widehat{g}$ divide $m$ ou a garantia de que tal elemento não existe.

A boa notícia é que estratégias desse tipo são possíveis. O Capitulo 5 se dedica a apresentar formas de se representar Bases de Gröbner infinitas por autômatos finitos e de utilizar essas representações para efetuar reduções. Entretanto, tais estratégias (e essa em particular) só são possíveis em contextos restritos.

As discussões que seguem mostrarão que não é possível encontrar um algoritmo que receba como entrada um conjunto gerador finito de um ideal $I$ e devolva uma representação com as propriedades acima mencionadas, no caso geral. Iniciamos com uma descrição mais precisa desse problema.

Definição 4.4.1 (Boa Representação de uma Base de Gröbner) Diremos que uma boa representação de uma Base de Gröbner $G$ consiste de uma representação finita $E$ de $G$ e um algoritmo $\mathcal{D}$, com a seguinte propriedade: ao receber um monômio $m$ e $E$ como entrada, $\mathcal{D}$ devolve um elemento $g$ de $G$ de modo que $\widehat{g}$ divide $m$ ou $\mathcal{D}$ fornece a garantia de que tal elemento não existe.

Problema da construção de Bases de Gröbner Seja $K\langle X\rangle$ uma álgebra livre e $X^{*}$ a base multiplicativa de $K\langle X\rangle$ composta por todas as palavras sobre $X$. Dados $K, X$, uma ordem admissível $\leq \mathrm{em} X^{*}$ e um conjunto finito de geradores para um ideal $I$ de $K\langle X\rangle$, devolva uma boa representação de uma Base de Gröbner $G$ para $I$ (com respeito a $\leq$ e $X^{*}$ ).

A primeira demonstração de que esse problema não é computável foi dada por Mora [Mor94] e o que faremos seguirá exatamente a mesma linha dessa demonstração.

Primeiramente, vale ressaltar que a demonstração feita aqui considerará o caso especial da álgebra livre (fixada a base $X^{*}$ ). Porém, como estas formam um caso particular das álgebras de caminhos (fixada a base $\mathcal{P}$ ), ao demonstrar que o problema da construção de Bases de Gröbner não é computável para as álgebras livres, fica evidente que ele também não é computável no caso mais geral.

\footnotetext{
${ }^{25}$ Note que isso é precisamente o que o Procedimento de Mora se propõe a fazer.
} 
A idéia principal da demonstração é que, se é possível calcular Bases de Gröbner para qualquer ideal de $K\langle X\rangle$, conseguimos resolver o problema da pertinência em $K\langle X\rangle$. Veremos então que isso nos leva a um algoritmo que resolve o problema da palavra para monóides finitamente gerados.

Esse último problema, por sua vez, é bastante estudado na literatura e sabidamente indecidível. Incluímos a seguir uma descrição formalizada desses dois importantes problemas para conveniência do leitor.

Problema da pertinência em $K\langle X\rangle$ Fixe um corpo $K$. Dados um alfabeto $X$, um conjunto finito de geradores para um ideal $I$ de $K\langle X\rangle$ e um elemento $f$ de $K\langle X\rangle$, decida se $f$ pertence a $I$.

Problema da palavra em monóides Seja $X$ um alfabeto e considere o monóide livre não comutativo gerado por $X, X^{*}$. Seja ainda

$$
\mathcal{R}=\left\{\left(l_{1}, r_{1}\right),\left(l_{2}, r_{2}\right), \ldots,\left(l_{k}, r_{k}\right)\right\} \subseteq X^{*} \times X^{*}
$$

Tome então $\equiv_{\mathcal{R}}$ a congruência em $X^{*}$ gerada por $\mathcal{R}$. Dados $X, \mathcal{R}$ e elementos $w_{1}$ e $w_{2}$ de $X^{*}$, decida se $w_{1} \equiv_{\mathcal{R}} w_{2}$.

Antes de prosseguirmos, vale comentar que, em 1958, Tsejtin [Tse58] forneceu um exemplo de um monóide $X^{*}$ e de um subconjunto $\mathcal{R}$ de $X^{*} \times X^{*}$, de modo que não é possível encontrar um algoritmo que decida se dois elementos de $X^{*}$ são equivalentes segundo $\equiv_{\mathcal{R}}$. Incluímos esse exemplo a seguir.

Exemplo 4.4.1 (Tsejtin, 1958) Seja $X^{*}$ o monóide livre não comutativo gerado por $\{a, b, c, d, e\}$. Tomemos

$$
\mathcal{R}=\{(a c, c a),(a d, d a),(b c, c b),(b d, d b),(e c a, c e),(e d b, d e),(c c a, c c a e)\}
$$

Então não existe um algoritmo que recebe como entrada $\mathcal{R}$ e dois elementos de $X^{*}$ e decide se eles são equivalentes por $\equiv_{\mathcal{R}}$. $\Delta$

Comentamos ainda que uma introdução mais detalhada a esse problema é dada em um livro de Lallement [Lali9]. O leitor interessado encontra nessa última referência uma discussão mais minuciosa do exemplo proposto por Tsejtin.

Vejamos então que o problema da palavra se reduz para o problema da pertinência. No que segue, considere $X$ um alfabeto qualquer, $X^{*}$ o monóide livre gerado por $X, \mathcal{R}=$ $\left\{\left(l_{1}, r_{1}\right),\left(l_{2}, r_{2}\right) \ldots,\left(l_{k}, r_{k}\right)\right\}$ um subconjunto de $X^{*} \times X^{*} \mathrm{e} \equiv_{\mathcal{R}}$ a congruência em $X^{*}$ gerada por $\mathcal{R}$.

Tome então $K$ um corpo arbitrário e considere $K\langle X\rangle$. Defina ainda $I$ como o ideal gerado por $\left\{l_{1}-r_{1}, l_{2}-r_{2}, \ldots l_{k}-r_{k}\right\}$. Então temos o seguinte lema (sua demonstração segue imediatamente da demonstração do Lema 3.4.19).

Lema 4.4.1 Dados dois elementos $w_{1}$ e $w_{2}$ de $X^{*}, w_{1} \equiv_{\mathcal{R}} w_{2}$ se e somente se $w_{1}-w_{2}$ pertence a $I$. 
Portanto, um algoritmo que resolva o problema da pertinência para $I$ em $K\langle X\rangle$, resolve o problema da palavra em $X^{*}$. Assim, chegamos à seguinte proposição.

\section{Proposição 4.4.2 Para todo corpo $K$, o problema da pertinência em $K\langle X\rangle$ é indecidível.}

Demonstração. Tome $K$ um corpo qualquer. Assuma que existe um algoritmo $\mathcal{A}$ que recebe como entrada um alfabeto $X$, um conjunto finito $F$ de elementos de $K\langle X\rangle$ e um elemento $f$ de $K\langle X\rangle$ e decide se $f$ pertence a $\langle F\rangle$.

Considere então uma instância do problema da palavra para monóides: tome um alfabeto $\Sigma$ e denote por $\Sigma^{*}$ o monóide livre não comutativo gerado por $\Sigma$. Tome ainda um subconjunto $\mathcal{R}=\left\{\left(l_{1}, r_{1}\right),\left(l_{2}, r_{2}\right), \ldots,\left(l_{k}, r_{k}\right)\right\}$ de $\Sigma^{*} \times \Sigma^{*}$ e dois elementos $w_{1}$ e $w_{2}$ de $\Sigma^{*}$.

Com isso, podemos reduzir o problema da palavra para o da pertinência da seguinte forma. Tome a álgebra livre $K\langle\Sigma\rangle$ e o subconjunto $F:=\left\{l_{1}-r_{1}, l_{2}-r_{2}, \ldots, l_{k}-r_{k}\right\}$. Então, pelo Lema 4.4 .1 o algoritmo $\mathcal{A}$, ao receber como entrada $\Sigma, F$ e $w_{1}-w_{2}$ decide se $w_{1} \equiv_{\mathcal{R}} w_{2}$.

Como tomamos uma instância qualquer do problema da palavra, chegamos a uma contradição com a suposição de o algoritmo $\mathcal{A}$ existir, o que nos mostra que o problema da pertinência é indecidível. Note ainda que, como tomamos $K$ qualquer, podemos concluir que esse problema é indecidível para todo corpo.

Pelas discussões do início da seção, o Algoritmo da Divisão pode ser facilmente modificado para receber como entrada uma boa representação de uma Base de Gröbner $G$, em vez do conjunto $G$ explícito. Não é difícil ver que a hipótese de o conjunto $S$ dado como entrada para esse algoritmo ser finito é usada exclusivamente para garantir a efetividade da execução da linha 4 do Algoritmo 4.1.1. Por outro lado, essa linha continua efetiva se utilizarmos uma boa representação para $S$ (seja ele finito ou não). Desse modo, essa versão modificada do Algoritmo da Divisão preserva as propriedades descritas nas Proposições 4.1.2 e 4.1.4.

Com isso, é claro que, dados um conjunto finito de geradores para um ideal $I$ de $K\langle X\rangle$ e um elemento $f$ de $K\langle X\rangle$, se é possível encontrar uma boa representação de uma Base de Gröbner $G$ para $I$ e computar a saída do Algoritmo da Divisão ao receber essa representação e o elemento $f$, então conseguimos decidir a pertinència de $f$ em $I$.

Portanto, alguma etapa desse processo deve ser não algorítmica. Voltamo-nos em seguida para encontrar quais etapas não podem ser computadas. Nosso objetivo é mostrar que (pelo menos para os casos de interesse) o único ponto de não efetividade existente no processo acima é a construção de uma boa representação para $G$. Examinemos primeiramente o Algoritmo da Divisão.

O leitor deve recordar de nossa discussão na Seção 4.1.2, que uma implementação do Algoritmo da Divisão em álgebras de caminhos (e é claro que o mesmo é válido para as álgebras livres) requer um corpo e uma ordem computáveis.

Mas, de acordo com a Proposição 4.4.2, o problema da pertinència é indecidível para todo corpo. Ainda, somos livres para escolher qualquer ordem admissivel em $X^{*}$ que desejarmos - e nenhuma escolha que fizermos pode permitir uma solução algorítmica para o problema da pertinència. Dessa maneira, assumiremos no que segue que temos um corpo $K$ e uma ordem admissível $\leq$ fixos e que 
ambos são computáveis. ${ }^{26}$

Logo, com essas hipóteses em mãos, podemos utilizar os comentários acima e a Proposição 4.1.13 para ver que a indecidibilidade do problema da pertinência se concentra justamente na impossibilidade de construção de boas representações de Bases de Gröbner. Assim, podemos concluir:

Teorema 4.4.3 Se $K$ e $\leq$ são computáveis, então o problema da construção de Bases de Gröbner não é computável.

Se retomarmos o conceito de Base de Gröbner truncada visto na Seção 4.1.1 (veja a Definição 4.1.2), chegamos a outro resultado.

Ao tomar um ideal $I$ e um elemento $f$ de $K\langle X\rangle$, o Corolário 4.1 .5 nos afirma que se tivermos em mãos um conjunto $S$ que seja uma Base de Gröbner para $I$ truncada em $\widehat{f}$, Divisão $(S, f)$ decide a pertinência de $f$ em $I$. Assim, podemos utilizar a indecidibilidade do problema da pertinência, a Proposição 4.1 .13 e um argumento análogo ao visto acima para estabelecer o seguinte resultado.

Proposição 4.4.4 O seguinte problema não é computável: dados um corpo $K$, uma ordem $\leq$ admissivel (ambos computáveis), um conjunto finito de geradores para um ideal I de $K\langle X\rangle$ e um elemento $f$ de $K\langle X\rangle$, devolva uma Base de Gröbner para I truncada em $\hat{f}$.

\footnotetext{
${ }^{26}$ Vale observar também que, se escolhemos um corpo ou uma ordem não computável, não conseguimos uma implementação efetiva do Algoritmo da Divisão. Com isso, o processo de resoluçāo da pertinência descrito acima continua não algorítmico no caso geral, independente de termos ou nāo boas representaçōes de Bases de Gröbner.
} 



\section{BASES DE GRÖBNER REGULARES}

Este capítulo traz uma introdução ao conceito de Base de Gröbner regular, apresentado por Mansson e Nordbeck em 2002 [MN02].

Vimos na Seção 4.4 que o problema de construir boas representações de Bases de Gröbner não é computável em geral. Contudo, essas representações podem ser encontradas em contextos mais restritos. Em essência, o que será desenvolvido neste capítulo apresenta um tipo de boa representação de Base de Gröbner (para um contexto restrito). Mencionaremos então como essa representação pode ser utilizada para efetuar reduções e algumas questões relacionadas à construção da própria representação.

Em particular, as Bases de Gröbner regulares serão introduzidas. De maneira resumida, elas constituem um tipo especial de Base de Gröbner para um ideal puramente binomial ${ }^{1}$ com a propriedade de que podem ser representadas por um autômato finito. Esse autômato pode então ser utilizado para efetuar reduções sobre essa base possivelmente infinita.

A Seção 5.1 apresenta algumas definições e conceitos iniciais e de motivação. Em seguida, na Seção 5.2 apresentamos brevemente o conceito de álgebra de autômato, que serviu de motivação para a introdução da noção de Base de Gröbner regular. A Seção 5.3 fornece uma introdução aos conceitos envolvendo relações racionais que serão necessários adiante. Em seguida, a Seção 5.4 apresenta a definição de Base de Gröbner regular e suas principais propriedades. Finalmente, nas Seções 5.5 e 5.6 discutimos algumas questões relacionadas ao uso de Bases de Gröbner regulares para efetuar reduções e apresentamos algumas considerações finais e problemas não resolvidos.

\subsection{Conceitos Iniciais}

Ao longo de todo este capítulo, nos restringiremos à álgebra livre $K\langle X\rangle$ com sua base multiplicativa $X^{*}$ fixada.

Vale ressaltar que o conceito de Base de Gröbner regular (que será definido na Seção 5.4) se aplica somente a ideais puramente binomiais. Ainda, veremos que esse conceito é definido somente para Bases de Gröbner compostas exclusivamente por binômios puros. Dessa maneira, antes de mais nada, mostramos que todo ideal puramente binomial possui uma Base de Gröbner puramente binomial.

Proposição 5.1.1 Todo ideal puramente binomial de $K\langle X\rangle$ admite uma Base de Gröbner composta somente por binômios puros.

\footnotetext{
${ }^{1}$ A definição de binòmio puro é fornecida na Definição 3.4 .2 .
} 
Antes de apresentar a demonstração dessa proposição, precisaremos de alguns resultados.

Afirmação 5.1.2 Sejam $f_{1}$ e $f_{2}$ binômios puros de $K\langle X\rangle$. Se

$$
s=l f_{1} r-l^{\prime} f_{2} r^{\prime}
$$

é um S-elemento envolvendo $f_{1}$ e $f_{2}$, então $s$ é nulo ou s é um binômio puro.

Demonstração. Sejam $f_{1}=a-b$ e $f_{2}=c-d$, com $a, b, c$ e $d$ em $X^{*}$. Então, $s=l^{\prime} d r^{\prime}-l b r$. Assim, temos duas possibilidades: ou $l b r$ é igual a $l^{\prime} d r^{\prime}$ ou esses monômios são distintos. ${ }^{2}$ No segundo caso, é imediato ver que $s$ é um binômio puro. Já no primeiro caso, $s$ é zero.

Um argumento análogo ao utilizado na demonstração da afirmação anterior nos leva ao seguinte resultado.

Afirmação 5.1.3 Sejam $f_{1}$ e $f_{2}$ dois binômios puros e $g$ um monômio em $K\langle X\rangle$. Então uma redução simples de $f_{1}$ por $f_{2}$ é um binômio puro ou é zero.

Ainda, uma redução simples $g^{\prime}$ de $g$ por $f_{2}$ é um monômio de forma que $\operatorname{cl}\left(g^{\prime}\right)=c l(g)$.

Antes de apresentar a demonstração para a Proposição 5.1.1, precisaremos de algumas propriedades do Algoritmo da Divisão.

Lema 5.1.4 Sejam $f$ um binômio puro de $K\langle X\rangle$ e $S$ um subconjunto puramente binomial de $K\langle X\rangle$. Então, a saída do Algoritmo da Divisão ao receber $f$ e $S$ como parâmetros é zero ou é um binômio puro.

Demonstração. Mostraremos que o Algoritmo da Divisão possui o seguinte invariante (ao receber $S$ e $f$ como parâmetros):

Invariante I No início de cada execução da linha 3 do algoritmo vale uma das três condições seguintes:

1. O valor de $g$ é um binômio puro e $h$ é zero.

2. O valor de $g$ é um monômio e $h$ é um monômio com $c l(h)=-c l(g)$.

3. O valor de $g$ é zero e $h$ ou é zero ou é um binômio puro.

No início da primeira execução dessa linha, $g=f$ e $h=0$, de modo que o invariante é verdadeiro. Assuma agora, por hipótese de indução, o invariante verdadeiro no início da $i$-ésima execução da linha 3 (e que não se trata da última execução dessa linha) e veremos que ele continua válido no início da $(i+1)$-ésima execuşão dessa linha.

Mas, no início da $i$-ésima execução da linha 3 , temos duas possibilidades: ${ }^{3}$

\footnotetext{
${ }^{2}$ Note que como se trata de dois monómios, não pode ocorrer de $l b r$ ser igual a $-l^{\prime} d r^{\prime}$.

${ }^{3}$ Note que a terceira condiçāo do Invariante $I$ não pode ser verdadeira neste ponto, uma vez que não se trata da última execução da linha 3 .
} 
- O valor da variável $g$ é um binômio puro e $h=0$.

Neste caso, a Afirmação 5.1.3 nos mostra que, ao final das linhas 4 e 5 o valor de $g$ será zero ou será um binômio puro. Se $g=0$, o valor de $h$ é zero e o invariante será satisfeito no início da $(i+1)$-ésima execução da linha 3.

Senão, uma simples inspeção na descrição do Algoritmo da Divisão nos mostra que as operações das linhas 7 e 8 cuidarão para que tanto $h$ quanto $g$ sejam monômios no início da $(i+1)$-ésima execução da linha 3 . Ainda, seus coeficientes líderes serão opostos pelo fato de $g$ ser um binômio puro. Portanto, o invariante continua válido no início da $(i+1)$-ésima execução da linha 3 .

- Os valores das variáveis $g$ e $h$ são monômios e $\operatorname{cl}(g)=-c l(h)$.

Pela Afirmação 5.1.3, ao final do laço das linhas 4 e $5, g$ será um monômio e $c l(g)$ será igual a $-c l(h)$. Note que a Afirmação 5.1 .3 nos garante que $g$ é não nulo neste ponto.

Então, as operações da linhas 7 e 8 farão com que $g$ receba zero e $h$ se torne um binômio puro. Então é claro ver que o invariante continuará verdadeiro no início da $(i+1)$-ésima execução da linha 3.

Com isso, o Invariante $I$ é válido no início da cada execução da linha 3. Em particular, ele é válido no início da última execução dessa linha. Mas nesse ponto, $g=0$ e vemos que $h$ é um binômio puro ou é zero, donde segue o resultado.

\section{Demonstração da Proposição 5.1.1.}

O resultado segue imediatamente da Afirmação 5.1.2, do Lema 5.1.4 e do funcionamento do procedimento PMORA.

Utilizando um argumento análogo ao visto na demonstração da proposição anterior, chegamos ao seguinte corolário.

Corolário 5.1.5 Se I é um ideal de KГ que possui um conjunto gerador puramente binomial $e$ uniforme, então I admite uma Base de Gröbner composta somente por binômios puros.

Podemos ainda utilizar o Corolário 5.1.5, a Afirmação 5.1.3 (na realidade sua generalização para álgebras de caminhos) e o Corolário 3.2.14 para ver o seguinte corolário.

Corolário 5.1.6 Seja I um ideal de K $\Gamma$ gerado por um conjunto puramente binomial e uniforme. Entäo a Base de Gröbner reduzida para I é puramente binomial.

Continuamos com a apresentação de mais um conceito que nos será útil adiante.

Dada uma linguagem $L \mathrm{em} X^{*}$, denotarmos por $\operatorname{red}(L)$ o conjunto formado pelos elementos de $L$ que não possuem divisores próprios dentre os elementos de $L$. Mais precisamente,

$$
\operatorname{red}(L):=\left\{u \in L \mid \text { se } u^{\prime} \in L \text { divide } u \text {, então } u^{\prime}=u\right\}
$$

O leitor atento irá notar que essa noção foi utilizada anteriormente no Lema 3.2.7 e na demonstração da Proposição 3.2.9 (de fato, o Lema 3.2.7, nos mostra que red $(L)$ é não vazio, para todo $L$ não vazio contido em $\left.X^{*}\right)$.

A seguinte propriedade envolvendo o conjunto $\operatorname{red}(L)$ será útil adiante. 
Lema 5.1.7 Se L é uma linguagem racional de $X^{*}$, então $\operatorname{red}(L)$ também é racional.

Demonstração. Tomamos $L$ uma linguagem racional. Se $L=\emptyset$, então $\operatorname{red}(L)=\emptyset \mathrm{e}$ segue o resultado. Suponha então $L \neq \emptyset$ e definimos:

$$
T:=X^{+} L X^{+} \cup L X^{+} \cup X^{+} L
$$

Como $L$ é racional, é claro pelas propriedades de fechamento de linguagens racionais que $T$ é uma linguagem racional (Proposição 1.5.1). Ainda, como as linguagens racionais são fechadas por diferença, segue que

$$
H:=L \backslash T
$$

é racional. Afirmamos então que $\operatorname{red}(L)=H$.

Para verificar essa igualdade, tomamos $h \mathrm{em} \operatorname{red}(L)$. Claro que $h$ pertence a $L$. Por outro lado, se assumimos que $h$ pertence a $T$, então existe um elemento de $L$ que divide propriamente $h$, nos levando a uma contradição com a hipótese de que $h$ pertence a $\operatorname{red}(L)$. Assim, concluímos que $\operatorname{red}(L) \subseteq H$.

Seja agora $h$ em $H$. Claro que $h$ pertence a $L$. Suponha então, por absurdo, que $h$ não pertence a $\operatorname{red}(L)$. Logo, existe $u \neq h$ em $L$, tal que $u$ divide $h$. Isso nos leva a três possibilidades ( $w$ e $w^{\prime}$ são elementos de $X^{+}$):

- $h=u w$, donde $h \in L X^{+} \subseteq T$, um absurdo.

- $h=w u$, donde $h \in X^{+} L \subseteq T$, um absurdo.

- $h=w u w^{\prime}$, donde $h \in x^{+} L X^{+} \subseteq T$, um absurdo.

Portanto, $H \subseteq \operatorname{red}(L)$, como queríamos.

Ao longo deste capítulo, dado um ideal $I$, denotaremos por $O_{I}$ (ou simplesmente por $O$, quando não houver possibilidade de confusão quanto ao ideal em questão) o conjunto gerador monomial minimal de $\langle\widehat{I}\rangle .{ }^{4}$ A existência e unicidade de $O_{I}$ são demonstradas na Proposição 3.2.9.

O leitor familiarizado com a definição de obstrução (obstruction, no original) utilizada por Ufnarovski irá notar pequena diferença entre essa última definição e a do conjunto $O$. Contudo, o Corolário 3.2.10 nos mostra que se trata do mesmo conjunto.

Mais que isso, dado um subconjunto $L$ de $X^{*}$, esse corolário e a definição de red $(L)$ nos mostram que

$$
\operatorname{red}(L)=O_{\langle\operatorname{red}(L)\rangle}=O_{\langle L\rangle}
$$

Ademais, se considerarmos o ideal $B$ de semigrupo gerado por $L$, então $B=X^{*}(\operatorname{red}(L)) X^{*}$.

Note que, se $G$ é uma Base de Gröbner para $I$, então $\langle\widehat{G}\rangle=\langle\widehat{I}\rangle$. Logo, $\operatorname{red}(\widehat{G})=O_{I}$. Assim. se $G$ é uma Base de Gröbner qualquer para um ideal $I$ de $K\langle X\rangle$,

\footnotetext{
[Anis6].

A notação $O$ refere-se ao termo obstruction, encontrado na literatura para denotar o conceito aqui definido
} 


$$
G^{\prime}:=\{g \in G \mid \widehat{g} \in \operatorname{red}(\widehat{G})\}
$$

é uma Base de Gröbner minimal para $I$.

\section{2 Álgebras de Autômato}

Esta seção traz o conceito de álgebra de autômato, introduzido por Ufnarovski em 1989 [Ufn89]. Trata-se de uma classe de álgebras com algumas boas propriedades, especialmente no que diz respeito a sua série de Hilbert. De fato, o principal interesse nessas álgebras está no estudo de séries de Hilbert.

Por outro lado, as álgebras de autômato serviram como uma motivação para a definição das Bases de Gröbner regulares e nosso interesse por essas álgebras se concentra nessa motivação. Dessa forma, faremos uma introdução bastante enxuta a elas nesta seção, deixando de lado algumas propriedades importantes. Em particular, não exploraremos sua relação com as séries de Hilbert, limitando-nos a referir o leitor interessado nesses aspectos para o artigo original de Ufnarovski.

Definição 5.2.1 (Álgebra de Autômato) Uma apresentação de uma álgebra da forma $A=$ $K\langle X\rangle / I$ é dita apresentação de autômato se existe alguma ordem admissivel (em $X^{*}$ ) de modo que o subconjunto Norm(I) formado pelos termos normais de $X^{*}$ com respeito a $I$ é uma linguagem racional sobre $X^{*}$.

Chamaremos uma álgebra de álgebra de autômato se ela possuir alguma apresentação de autômato.

Apresentamos em seguida alguns resultados relacionados com álgebras de autômato. Para os resultados, fixamos uma ordem admissível qualquer $\leq$ em $X^{*}$ e tomamos um ideal $I$ de $K\langle X\rangle$.

O seguinte resultado é o Teorema 7 de [Ufn89]. A demonstração apresentada aqui não se encontra na literatura e, ao que tudo indica, é mais simples que a original.

Proposição 5.2.1 (Ufnarovski, 1989) Dados um ideal $I$ de $K\langle X\rangle$ e uma ordem admissivel $\leq$ em $X^{*}$, Norm(I) é racional se e somente se $O_{I}$ é racional.

Demonstração. Denotamos por $O=O_{I}$. Primeiramente, note que, pela definição de termo normal (Definição 3.1.3), temos que $X^{*}=\widehat{I} \cup \operatorname{Norm}(\mathrm{I})$. Ademais, não é difícil ver que $\widehat{I}$ é um ideal de semigrupo do monóide $X^{*}$ e que $\widehat{I}$ é gerado por $O$ (como ideal de semigrupo). De fato, $\widehat{I}=X^{*} O X^{*}$ e $O=\operatorname{red}(\widehat{I})$, pelo Corolário 3.2.10.

Logo, se $O$ é racional,

$$
\operatorname{Norm}(\mathrm{I})=X^{*} \backslash \widehat{I}=X^{*} \backslash\left(X^{*} O X^{*}\right)
$$

também é racional.

Por outro lado, se Norm(I) é racional, então

$$
O=\operatorname{red}(\widehat{I})=\operatorname{red}\left(X^{*} \backslash \operatorname{Norm}(\mathrm{I})\right)
$$

também o é, pelo Lema 5.1.7. 
Corolário 5.2.2 Se G é uma Base de Gröbner para I, tal que ĜG é racional, então Norm(I) também é racional.

Demonstração. Primeiro notamos que, pelos Corolários 3.2 .14 e 3.2 .15 , segue que $O \subseteq \widehat{G}$. Ainda, pelo Corolário 3.2 .10 , segue que $O=\operatorname{red}(\widehat{G})$. O resultado segue então do Lema 5.1.7 e da Proposição 5.2.1.

Em 1995 Ufnarovski conjeturou que a propriedade de uma álgebra ser de autômato depende da apresentação da álgebra. Um exemplo que mostra esse fato foi dado por Mansson e Nordbeck em 2002 [MN02]:

Exemplo 5.2.1 (Mansson e Nordbeck, 2002) Consideramos $X$ o alfabeto dado por $X=\{a, b, x, y\}$, a álgebra livre $K\langle X\rangle$ (com sua base multiplicativa $X^{*}$ ) e o ideal $I$ de $K\langle X\rangle$, gerado por

$$
G:=\left\{a x^{k} y^{k} b \mid k \geq 1\right\}
$$

Como o ideal $I$ é monomial, é imediato ver que todas as relações de sobreposição envolvendo elementos de $G$ são iguais a zero. Ainda, não é difícil ver que, dados dois elementos distintos de $\widehat{G}$, um não divide o outro. Logo, pelo Teorema 4.2 .31 e pelo Corolário 3.2.10, vemos que $G$ é a Base de Gröbner reduzida para $I$ (para qualquer ordem admissível fixada em $X^{*}$ ). Assim, $G=\widehat{G}=O$.

Por meio do Lema da Iteração (Proposição 1.5.5), não é difícil ver que $\widehat{G}$ não é racional. Logo, pelas igualdades acima e pela Proposição 5.2.1, segue que a apresentação $K\langle X\rangle / I$ não é de autômato.

Por outro lado, podemos definir a aplicação

$$
\alpha: X \longrightarrow K\langle X\rangle
$$

dada por:

$$
\begin{array}{ll}
\alpha(a)=a & \alpha(b)=b \\
\alpha(x)=x & \alpha(y)=x+y
\end{array}
$$

É claro que $\alpha$ admite uma única extensão $\alpha^{\prime}$ para um homomorfismo de $X^{*}$ em $K\langle X\rangle$. A aplicação $\alpha^{\prime}$, por sua vez, admite uma única extensão $K$-linear de $K\langle X\rangle$ em $K\langle X\rangle$, que denotamos por $\varphi$.

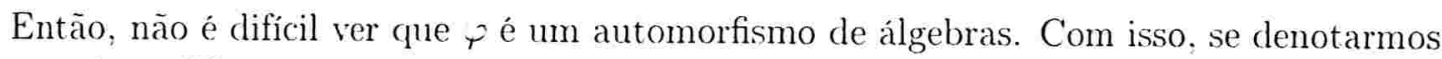
por $J=\varphi(I)$, teremos o seguinte isomorfismo:

$$
K\langle X\rangle / I \cong K\langle X\rangle / J
$$

(o isomorfismo pode ser verificado facilmente definindo uma aplicação que associa cada elemento $a+I$ de $K\langle X\rangle / I$ a $\varphi(a)+J$ em $K\langle X\rangle / J)$. 
Com isso, o ideal $J$ é gerado por $G^{\prime}:=\varphi(G)=\left\{g_{k} \mid k \geq 1\right\}$, com

$$
g_{k}=a x^{k}(x+y)^{k} b=a x^{2 k} b+a x^{2 k-1} y b+\cdots a x^{k} y^{k} b
$$

para todo $k \geq 1$.

Assim, se tomarmos a ordem de grau e lexicografia em $X^{*} \operatorname{com} x>y$, teremos $\widehat{G^{\prime}}=$ $\left\{a x^{2 k} b \mid k \geq 1\right\}$, donde $\widehat{G^{\prime}}=a\left(x^{2}\right)^{+} b$ é um conjunto regular. Logo, segue do Corolário 5.2.2 que a apresentação $K\langle X\rangle / J$ é de autômato.

Finalmente, note que se tomarmos a ordem deglex com $y>x$, o conjunto dos termos líderes de $G^{\prime}$ será $\left\{a x^{k} y^{k} b \mid k \geq 1\right\}$. Com isso, não é difícil ver que cada elemento $g_{k}$ é reduzido. Conforme comentado antes, não existem dois elementos distintos no conjunto $\left\{a x^{k} y^{k} b \mid k \geq 1\right\}$, de maneira que um divida o outro.

Logo, $G^{\prime}$ é a Base de Gröbner reduzida para $J$ (nessa ordem). Portanto, $O=\left\{a x^{k} y^{k} b \mid\right.$ $k \geq 1$ \} e concluímos que Norm(I) não é racional, pela Proposição 5.2.1.

Assim, vemos que uma álgebra pode ser de autômato, mas possuir apresentações que não são de autômato. Ainda, uma dada apresentação pode possuir um conjunto racional de termos normais para uma escolha de ordem admissível em $X^{*}$ e um conjunto não racional de termos normais para um escolha diferente de ordem.

Encerramos esta seção comentando que foi demonstrado por Ufnarovski (Teorema 9 de [Ufn89]) que as seguintes classes de álgebras finitamente geradas são álgebras de autômato: álgebras comutativas, álgebras definidas por relações homogêneas de grau dois, álgebras monomiais e álgebras cujas relações são determinadas pela comutatividade de alguns geradores.

\subsection{Relações Racionais}

Nesta seção apresentaremos algumas definições e resultados básicos sobre relações racionais e transdutores. Elas formam uma subárea bastante extensa da Teoria de Linguagens Formais e Autômatos, de modo que não pretendemos abordar nenhum desses conceitos com profundidade. Entretanto, eles serão necessários para discussões e definições envolvendo as Bases de Gröbner regulares. As demonstrações e detalhes não explorados aqui podem ser vistos nos livros de Berstel [Ber79] e Eilenberg [Eil74]. Outra boa introdução ao assunto é dada por Souza [dS04].

Antes de apresentar as relações racionais propriamente ditas, precisamos de uma generalização dos conceitos vistos na Seção 1.5. Lá, abordamos o caso mais conhecido do monóide livre. Apresentaremos alguns elementos da generalização dessa teoria para monóides quaisquer.

Essa generalização será necessária somente para introduzirmos o conceito de relações racionais. Vale comentar, contudo, que ao fazermos essa generalização, propriedades importantes existentes para o monóide livre são perdidas ${ }^{5}$, de modo que o caso mais geral se torna sensivelmente mais complexo.

No que segue, seja então M um monóide qualquer. Como se trata de um semigrupo, dados subconjuntos $A$ e $A^{\prime}$ de $M$, seu produto está definido como:

\footnotetext{
${ }^{5}$ Aqui podemos destacar o Teorema de Kleene (Teorema 1.5.4): ele não é válido no contexto de um monóide qualquer.
} 


$$
A A^{\prime}:=\left\{u v \in M \mid u \in A \text { e } v \in A^{\prime}\right\}
$$

Assim, podemos definir as operações racionais de maneira completamente análoga ao que foi feito na Seção 1.5. Ainda, podemos definir a noção de subconjunto racional de $M$, de forma totalmente equivalente ao que é comumente feito para o monóide livre:

Definição 5.3.1 (Conjunto Racional) Um subconjunto $A$ de um monóide $M$ é dito racional se ele pode ser obtido a partir dos conjuntos unitários $\{m\}$, com $m$ em $M$ e do conjunto vazio através de um número finito de aplicações das operações união, produto e estrela.

Ainda, denotaremos a família de todos os subconjuntos racionais de $M$ por $\operatorname{Rac}(M)$.

Não é difícil ver que o conceito de expressão racional se transfere sem modificações para o contexto de um monóide qualquer.

No restante do texto, não faremos uso do conceito de conjunto reconhecível em um monóide qualquer. Entretanto, incluímos aqui sua definição para conveniência do leitor. Afirmamos ainda, sem demonstração, que essa definição é equivalente à Definição 1.5.3 no caso em que $M$ é um monóide livre. Contudo, é importante ressaltar que o Teorema de Kleene (Teorema 1.5.4) não é válido nesse contexto mais geral. ${ }^{6}$

Definição 5.3.2 (Conjunto Reconhecivel) Seja $M$ um monóide qualquer. Um subconjunto $A$ de $M$ é reconhecível se existe um monóide finito $N$, um homomorfismo $\alpha: M \longrightarrow N$ e um subconjunto $P$ de $N$ de modo que $A=\alpha^{-1}(P)$.

O seguinte resultado é a Proposição 2.2 de [Ber79] e nos será valioso na Seção 5.4 .

Proposição 5.3.1 Sejam $M$ e $M^{\prime}$ monóides, $\alpha: M \longrightarrow M^{\prime}$ um homomorfismo de monóides $e$ $S$ um subconjunto racional de $M$. Então, $\alpha(S)$ é um subconjunto racional de $M^{\prime}$.

Ainda, se $\alpha$ é um homomorfismo sobrejetor e $R$ é um subconjunto racional de $M^{\prime}$, então existe um subconjunto racional $A$ de $M$, tal que $\alpha(A)=R$.

Demonstração. Seja $H$ o conjunto de todos os subconjuntos $A$ de $M$ que satisfazem o seguinte: $\alpha(A)$ é um subconjunto racional de $M^{\prime}$. Mostraremos que $H$ contém todos os subconjuntos racionais de $M$.

Claro que $\alpha(\emptyset)=\emptyset$ e $\alpha(m)=m^{\prime}$, para todo $m$ em $M$, de modo que o conjunto vazio e os conjuntos unitários pertencem a $H$. Ademais, se $A$ e $B$ pertencem a $H$, então $\alpha(A \cup B)=\alpha(A) \cup \alpha(B)$. Mas $\alpha(A)$ e $\alpha(B)$ são subconjuntos racionais de $M^{\prime}$ (pelo fato de $A$ e $B$ pertencerem a $H)$, donde concluímos que $\alpha(A \cup B) \in \operatorname{Rac}\left(M^{\prime}\right)$.

Analogamente, vemos que

$$
\begin{aligned}
& \alpha(A B)=\alpha(A) \alpha(B) \text { pertence a } \operatorname{Rac}\left(M^{\prime}\right) \mathrm{e} \\
& \alpha\left(A^{*}\right)=(\alpha(A))^{*} \text { pertence a } \operatorname{Rac}\left(M^{\prime}\right)
\end{aligned}
$$

\footnotetext{
${ }^{6} \mathrm{Lm}$ exemplo que mostra esse fato pode ser visto no livro de Berstel [Ber $\bar{\imath} 9$ ].
} 
Assim, $\operatorname{Rac}(M)$ é um subconjunto de $H$, o que demonstra a primeira parte da proposição. Para a segunda parte, seja $J$ o conjunto formado por todos os subconjuntos $A$ de $M^{\prime}$ que satisfazem: existe $B$ em $\operatorname{Rac}(M)$, tal que $\alpha(B)=A$. Mostraremos que $\operatorname{Rac}\left(M^{\prime}\right) \subseteq J$. Mas como $\alpha$ é sobrejetora, segue que, para todo $m$ em $M^{\prime}$, existe $a$ em $M$ tal que $\alpha(a)=m$. Logo os conjuntos unitários pertencem a $J$. Ainda, é claro que $\alpha(\emptyset)=\emptyset$, donde o conjunto vazio também pertence a $J$. O resultado segue então por um argumento análogo ao visto para a primeira parte.

Apresentamos em seguida a generalização do conceito de autômato para o contexto de um monóide qualquer.

Definição 5.3.3 (M-Autômato) Um $M$-autômato (ou autômato sobre $M$ ) é uma máquina $\mathcal{A}=(Q, M, I, T, E)$ composta de:

- Um monóide $M$.

- Um conjunto finito $Q$ de estados.

- Subconjuntos $I$ e $T$ de $Q$ de estados iniciais e finais, respectivamente.

- Um conjunto finito $E \subseteq Q \times M \times Q$ de transições.

Note que a única diferença de um $M$-autômato para um autômato sobre um monóide livre é o fato de que os arcos do $M$-autômato são rotulados por elementos do monóide $M$, em vez de rotulados por letras. Comentamos ainda que a definição de $M$-autômato exige que seu grafo seja finito.

Dessa forma, os conceitos de caminho bem-sucedido e de rótulo de um caminho (com a devida modificação para que consideremos o produto dos rótulos dos arcos que compõem o caminho e não mais sua concatenação) podem ser transportados para este contexto sem modificações. Lembramos que o comportamento de um $M$-autômato $\mathcal{A}$ é o conjunto formado pelos rótulos dos caminhos bem-sucedidos em $\mathcal{A}$. Ainda, dizemos que dois $M$-autômatos são equivalentes se possuírem o mesmo comportamento.

Em seguida, nos voltamos para demonstrar que todo subconjunto racional de $M$ é o comportamento de um $M$-autômato. Antes, contudo, precisaremos de um resultado.

Proposição 5.3.2 Um subconjunto $A$ de um monóide $M$ é racional se e somente se existem um alfabeto $X$, uma linguagem racional $L$ sobre $X$ e um homomorfismo de monóides $\quad \varphi: X^{*} \longrightarrow M$ tal que $\varphi(L)=A$.

Demonstração. Primeiro, é claro que se existem $X, L$ e $\varphi$ nas condições acima, $A$ é um conjunto racional de $M$ pela Proposição 5.3.1.

Para ver a implicação contrária, utilizaremos uma técnica similar ao que foi feito na demonstração da Proposição 5.3.1. Assim, considere $H$ o conjunto formado por todos os subconjuntos de $M$ que satisfazem a proposição. Em outras palavras, se $A$ pertence a $H$, então existem um alfabeto $X$, uma linguagem racional $L$ sobre $X$ e um homomorfismo de monóides $\varphi$ de $X^{*}$ em $M \operatorname{com} \varphi(L)=A$. Mostraremos então que $\operatorname{Rac}(M)$ está contido em $H$. 
Vejamos primeiro que os conjuntos unitários pertencem a $H$. Assim, tomamos $\{m\} \subseteq M$. Podemos então tomar um alfabeto $X$, contendo apenas um símbolo $x$ e definir $\varphi(x)=m$. Claro que, uma vez que definimos o valor de $\varphi$ nos elementos de $X$, o homomorfismo de $X^{*}$ em $M$ está completamente definido. Mas assim, $\{x\}$ é um subconjunto racional de $X^{*}$ e $\varphi(x)=m$. Logo, $\{m\} \in H$, para todo $m$ em $M$. Ademais, note que $\varphi(\emptyset)=\emptyset$, donde concluímos que $\emptyset \in H$.

Vejamos agora que $H$ é fechado pelas operações racionais. Para tanto, tomamos $A$ e $B$ em $H$. Então existem um alfabeto $X$, uma linguagem racional $L$ sobre $X$ e um homomorfismo $\varphi: X^{*} \longrightarrow M$ tal que $\varphi(L)=A$. Ademais, existe um alfabeto $\Sigma$, uma linguagem racional $L^{\prime}$ sobre $\Sigma$ e um homomorfismo de monóides $\alpha: \Sigma^{*} \longrightarrow M$, tal que $\alpha\left(L^{\prime}\right)=B$.

Note que podemos assumir, sem perda de generalidade, que os alfabetos $X$ e $\Sigma$ são conjuntos disjuntos. ${ }^{7}$ Consideramos então o alfabeto $(X \cup \Sigma)$ e o seguinte homomorfismo de monóides

$$
\beta:(X \cup \Sigma)^{*} \longrightarrow M
$$

definido da seguinte maneira por sua imagem nas letras $(y \in X \cup \Sigma)$ :

$$
\begin{aligned}
& \beta(y)=\varphi(y), \text { se } y \text { pertence a } X \text { e } \\
& \beta(y)=\alpha(y), \text { se } y \text { pertence a } \Sigma .
\end{aligned}
$$

Então, não é difícil ver que

$$
\begin{aligned}
& \beta\left(L \cup L^{\prime}\right)=A \cup B, \\
& \beta\left(L L^{\prime}\right)=A B \mathrm{e} \\
& \beta\left(L^{*}\right)=(\beta(L))^{*}=A^{*} .
\end{aligned}
$$

Logo, $H$ é fechado pelas operações racionais e podemos concluir que $\operatorname{Rac}(M) \subseteq H$, como queríamos.

Proposição 5.3.3 Um subconjunto $A$ de um monóide $M$ é racional se e somente se $A$ é o comportamento de um $M$-autômato.

Demonstração. Tomamos inicialmente $R$ em $\operatorname{Rac}(M)$. Mas então, pela Proposição j.3.2, existem um alfabeto $X$, uma linguagem racional $L$ sobre $X$ e um homomorfismo de monóides $\varphi$ de $X^{*}$ em $M$ tal que $\varphi(L)=R$.

Pelo Teorema de Kleene (Teorema 1.5.4), $L$ é reconhecida por algum $X$-autômato $\mathcal{A}$. Vejamos então como construir um $M$-autômato $\mathcal{B}$, a partir de $\mathcal{A}$, de modo que $R=|\mathcal{B}|$. Para tanto, tomamos $\mathcal{B}$ com o mesmo conjunto de estados de $\mathcal{A}$ e mesmos conjuntos de estados iniciais e finais. Ainda, para cada transição $\left(q_{1}, u, q_{2}\right)$ em $\mathcal{A}$, inserimos uma transição em $\mathcal{B}$ da forma $\left(q_{1}: \varphi(u), q_{2}\right)$. É claro que $\mathcal{B}$ é um $M$-autómato. Resta mostrar que seu comportamento é o conjunto $R$.

\footnotetext{
${ }^{7}$ Se näo forem, basta tomar outro alfabeto $\Sigma^{\prime}$, disjunto de $X$, de modo que card $(\Sigma)=\operatorname{card}\left(\Sigma^{\prime}\right)$. Então os monóides $\Sigma^{*}$ e $\Sigma^{\prime *}$ sĩo isomorfos.
} 
Mas então, se tomarmos $m$ em $R$, do fato de $R=\varphi(L)$, segue que existe $u$ em $L$ com $\varphi(u)=m$. Assim, como $u$ pertence a $L$, existe um passeio bem-sucedido em $\mathcal{A}$ que soletra $u$. Mas então, pela construção de $\mathcal{B}$ e pelo fato de $\varphi$ ser um homomorfismo, segue que existe um passeio bem-sucedido em $\mathcal{B}$ que soletra $m$. Logo, concluímos que $R \subseteq|\mathcal{B}|$.

Por outro lado, se $m$ pertence a $|\mathcal{B}|$, então existe um passeio bem-sucedido em $\mathcal{B}$ que soletra $m$. Assim, pela construção de $\mathcal{B}$, é fácil ver que existe $a_{1} a_{2} \cdots a_{n}$ em $L$ tal que:

$$
m=\varphi\left(a_{1}\right) \varphi\left(a_{2}\right) \cdots \varphi\left(a_{n}\right)=\varphi\left(a_{1} a_{2} \cdots a_{n}\right) \in \varphi(L)=R
$$

concluindo essa parte da prova.

Para ver a implicação reversa, tomemos um $M$-autômato $\mathcal{B}=(Q, M, I, T, S)$. Consideramos então cada transição de $\mathcal{B}$ como uma letra e chamamos o conjunto dessas transições de $E$. Tomamos então o monóide livre gerado por essas transições, $E^{*}$. Construiremos um $E$-autômato $\mathcal{A}$, a partir de $\mathcal{B}$, da seguinte forma. O autômato $\mathcal{A}$ possui o mesmo conjunto de estados que $\mathcal{B}$, bem como os mesmos conjuntos de estados iniciais e finais. Ainda, para cada transição $e=(p, m, q)$ de $\mathcal{B}$, adicionamos uma transição $(p, e, q)$ em $\mathcal{A}$ (note que, de fato, os $\operatorname{arcos}$ de $\mathcal{A}$ são rotulados por elementos de $E$ ).

Definimos agora a aplicação $\alpha: E \longrightarrow M$, da seguinte forma: $\alpha(e)=|e|$. Claro que $\alpha$ pode ser estendida de maneira única a um homomorfismo de $E^{*}$ em $M$ (também denotaremos por $\alpha$ essa extensão).

Mas então, pelo Teorema de Kleene, temos que $|\mathcal{A}|$ é uma linguagem racional. Agora, pela construção de $\mathcal{A}$ e pela definição de $\alpha$ não é difícil ver que $\alpha(|\mathcal{A}|)=|\mathcal{B}|$. O resultado segue então da Proposição 5.3.1.

Um $M$-autômato $\mathcal{A}=(Q, M, I, T, E)$ é normalizado se $I=\{i\}, T=\{t\}, i$ é distinto de $t$, não existe nenhum arco em $\mathcal{A}$ com ponta final $i$ e nenhum arco em $\mathcal{A}$ com ponta inicial $t$.

Proposição 5.3.4 Todo $M$-autômato é equivalente a um $M$-autômato normalizado.

Demonstração. Dado um $M$-autômato $\mathcal{A}=(Q, M, I, T, E)$, podemos construir um $M$-autômato $\mathcal{B}=\left(Q \cup\{i, t\}, M,\{i\},\{t\}, E^{\prime}\right)$, normalizado e equivalente a $\mathcal{A}$, da seguinte maneira. ${ }^{8}$ Para cada $q$ em $I$, acrescentamos um arco com ponta inicial $i$ e ponta final $q$, rotulado por $1_{M}$. Analogamente, para cada $q$ em $T$, acrescentamos um arco com ponta inicial $q$ e ponta final $t$ rotulado por $1_{M}$.

$A$ ssim, não é difícil ver que $|\mathcal{B}|=|\mathcal{A}|$ e que $\mathcal{B}$ é normalizado.

Em seguida, apresentamos o conceito de monóide produto, que será essencial para definir o conceito de relação racional.

Dados dois monóides $M$ e $N$, o monóide produto de $M$ por $N$ é o monóide dado pelo conjunto $M \times N$ e pela multiplicação coordenada a coordenada. Assim,

$$
(m, n) \cdot\left(m^{\prime}, n^{\prime}\right):=\left(m \cdot M m^{\prime}, n \cdot N n^{\prime}\right), \text { para todos }(m, n) \text { e }\left(m^{\prime}, n^{\prime}\right) \text { em } M \times N
$$

\footnotetext{
${ }^{8}$ Assumimos que $i$ e $t$ são distintos e que eles não pertencem a $Q$.
} 
Não é difícil ver que se trata de um monóide com unidade $\left(1_{M}, 1_{N}\right)$. Com isso, os conceitos vistos até aqui se transferem sem modificações para monóides produto.

As operações racionais descritas anteriormente também se transferem sem modificações para este contexto. Contudo, exibimo-nas explicitamente em seguida, para conveniência do leitor (sejam $R$ e $R^{\prime}$ subconjuntos de $\left.M \times N\right)$ :

$$
\begin{aligned}
& R \cup R^{\prime}=\left\{(m, n) \mid(m, n) \in R \text { ou }(m, n) \in R^{\prime}\right\} \\
& R R^{\prime}=\left\{\left(m m^{\prime}, n n^{\prime}\right) \mid(m, n) \in R \text { e }\left(m^{\prime}, n^{\prime}\right) \in R^{\prime}\right\} \\
& R^{*}=\left\{\left(m_{1} \cdots m_{k}, n_{1} \cdots n_{k}\right) \mid\left(m_{1}, n_{1}\right), \ldots,\left(m_{k}, n_{k}\right) \in R, \text { para todo } k \geq 1\right\} \cup\left\{\left(1_{M}, 1_{N}\right)\right\}
\end{aligned}
$$

Com isso, podemos apresentar a definição de relação racional.

Definição 5.3.4 (Relação Racional) Um subconjunto $R$ de um monóide produto $M \times N$ é chamado de uma relação racional (ou transdução) sobre $M$ e $N$ (ou de $M$ para $N$ ) se $R$ for um subconjunto racional do monóide produto $M \times N$.

Vale lembrar que as relações (racionais ou não) de $M$ para $N$ podem ser vistas de duas formas: de uma maneira mais "estática" como um subconjunto do produto cartesiano $M \times N$ ou de uma maneira mais "dinâmica" como uma função de $M$ em $\mathcal{P}(N)$. Comumente na literatura, o termo "relação (racional)" se refere a essa visão mais dinâmica e o termo gráfico é utilizado para denotar o subconjunto do produto cartesiano correspondente à relação. Em nossa abordagem, não faremos distinção entre essas duas formas de ver relações.

Definição 5.3.5 (Transdutor) Sejam $M$ e $N$ dois monóides. Um $M$ - $N$-transdutor (ou transdutor sobre $M$ e $N$ ) é um autômato sobre o monóide produto $M \times N$.

A Proposição 5.3.3 nos diz que uma relação é racional se e somente se for o comportamento de algum transdutor.

Informalmente, um $M-N$-transdutor pode ser visto de duas maneiras. Primeiramente, como um reconhecedor de conjuntos que lê elementos compostos por pares ordenados em $M \times N$ (ou que lê elementos de $M$ e de $N$ a partir de duas fitas de entrada). Uma segunda maneira de ver transdutores é encará-los como máquinas que realizam uma relação racional $R$. De acordo com essa visão, um transdutor lê elementos de $M$ de uma fita de entrada e escreve uma saída (algumas vezes chamada de emissão) consistindo de conjuntos de elementos de $N$ em uma fita de saída. O conteúdo da fita de saída é a imagem por $R$ do elemento lido da fita de entrada se e somente se o transdutor se encontra em um estado final ao término da computação (o Exemplo 5.3.1 ilustrará um pouco melhor esta discussão). Em seguida, seremos mais precisos.

Seguindo a notação vista para autômatos em geral, se $\mathcal{T}=(Q, M \times N, I, T, E)$ é um $M$ - $N$ transdutor, então $|\mathcal{T}|$ denota o comportamento de $\mathcal{T}$, que será uma relação racional. Então, se $m$ pertence a $M$,

$$
|\mathcal{T}|(m)=\{n \in N|(m, n) \in| \mathcal{T} \mid\}
$$

Em outras palavras, $|\mathcal{T}|(m)$ é a imagem de $m$ pela relação reconhecida por $\mathcal{T}$.

Diante dessa discussão, $M$ (resp. $N$ ) é algumas vezes chamado de monóide de entrada (resp. monóide de saída) de $\mathcal{T}$. 
Nas seções seguintes, trabalhemos com monóides produto da forma $X^{*} \times \Sigma^{*}$. É claro que se trata de um caso particular do que foi abordado até aqui, de modo que todo o conteúdo dessa seção se aplica a esse caso sem modificações.

Seguiremos a notação comumente vista na literatura e diremos que transdutores sobre $X^{*}$ e $\Sigma^{*}$ são $X$ - $\Sigma$-transdutores. Nesta caso, diremos que $X$ (resp. $\Sigma$ ) é o alfabeto de entrada (resp. alfabeto de saída) de $\mathcal{T}$.

Chamamos a atenção do leitor para o fato de que, em geral, as transições em um $X$ - $\Sigma$-transdutor são rotuladas por duas palavras e não duas letras.

Com respeito à representação gráfica de transdutores, é comum denotar rótulos das transições de um transdutor sobre $M$ e $N$ por $m / n$, em vez de $(m, n)$.

Exemplo 5.3.1 Considere o $X$ - $X$-transdutor $\mathcal{A}=\left(Q, X^{*} \times X^{*}, I, F, E\right)$ dado pela figura abaixo.

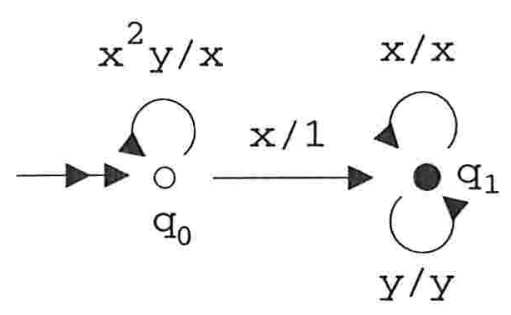

No exemplo, $X=\{x, y\}, Q=\left\{q_{0}, q_{1}\right\}, I=\left\{q_{0}\right\}, F=\left\{q_{1}\right\}$ e o conjunto de transições $E$ é dado por:

$$
E=\left\{\left(q_{0},\left(x^{2} y, x\right), q_{0}\right),\left(q_{0},(x, 1), q_{1}\right),\left(q_{1},(x, x), q_{1}\right),\left(q_{1},(y, y), q_{1}\right)\right\}
$$

A relação racional aceita por $\mathcal{T}$ é: $\left(x^{2} y, x\right)^{*}(x, 1)((x, x) \cup(y, y))^{*} \subseteq X^{*} \times X^{*}$. Ela é dada por $\left(u \in X^{*}\right)$ :

$$
|\mathcal{T}|(u)=\left\{\begin{array}{l}
\left\{x^{k} w\right\}, \text { se } u=\left(x^{2} y\right)^{k} x w, \text { para algum } k \geq 0 \text { e algum } w \in X^{*} \\
\emptyset, \text { se } u \notin\left(x^{2} y\right)^{*} x X^{*}
\end{array}\right.
$$

\subsection{Bases de Gröbner Regulares}

Apresentamos nesta seção o conceito de Base de Gröbner regular. Lembramos que nosso interesse está em encontrar boas representações de Bases de Gröbner. Nesse sentido, as Bases de Gröbner regulares consistem de Bases de Gröbner que podem ser representadas por relações racionais e, portanto, possuem uma representação finita (e "computacionalmente amigável") por meio de um transdutor.

A fim de ilustrar nossos objetivos, fornecemos um exemplo de motivação.

Exemplo 5.4.1 Considere o ideal $I:=\left\langle x^{2}-x y\right\rangle$ de $K\langle x, y\rangle$ e a ordem de grau e lexicografia em $X^{*} \operatorname{com} x>y$. 
O Exemplo 4.3.2 nos mostrou que uma Base de Gröbner para $I$ nesse caso é:

$$
G:=\left\{x y^{k} x-x y^{k+1} \mid k \geq 0\right\}
$$

A fim de representar a Base de Gröbner $G$ como uma relação racional, consideramos o monóide produto $M:=X^{*} \times X^{*}$ e identificamos elementos de $G$ com elementos de $M$, da seguinte maneira:

$$
x y^{k} x-x y^{k+1} \leftrightarrow(x, x)(y, y)^{k}(x, y)
$$

Assim, $G$ pode ser identificada com a relação racional $R:=(x, x)(y, y)^{*}(x, y)$. O transdutor que realiza $R$ é apresentado abaixo.

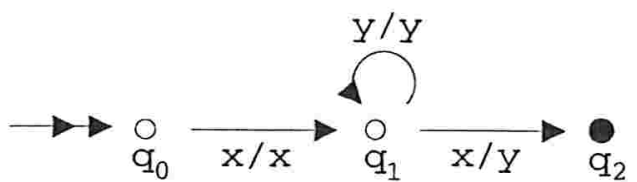

Lembramos que, ao longo de todo este capítulo, temos trabalhado apenas com a álgebra livre. Assim, seja $X$ um alfabeto, $K\langle X\rangle$ a álgebra livre sobre $X$ e fixemos a base $X^{*}$ de $K\langle X\rangle$ e uma ordem admissível $\leq$ em $X^{*}$. Considere ainda o monóide produto $M:=X^{*} \times X^{*}$. Definimos então a aplicação

$$
f: M \longrightarrow K\langle X\rangle
$$

por

$$
f\left(\left(m, m^{\prime}\right)\right):=m-m^{\prime}
$$

e as "projeções"

$$
f_{1}: M \longrightarrow X^{*} \quad \text { e } \quad f_{2}: M \longrightarrow X^{*}
$$

por

$$
f_{1}\left(\left(m, m^{\prime}\right)\right):=m \quad \text { e } \quad f_{2}\left(\left(m, m^{\prime}\right)\right):=m^{\prime}
$$

Não é difícil ver que o subconjunto de $M$ com imagem nula pela aplicação $f$ é $D_{M}$ (a diagonal de $M$, vide Seção 1.1) e que as aplicações $f_{1}$ e $f_{2}$ são homomorfismos de monóides. ${ }^{9}$ Ademais, a Proposição 5.3.1 nos mostra que se $R \subseteq M$ é uma relação racional, então $f_{1}(R)$ e $f_{2}(R)$ são linguagens racionais sobre $X$.

Com isso, podemos apresentar a definição formal de Base de Gröbner regular. ${ }^{10}$

\footnotetext{
'As aplicaçōes $f_{1}$ e $f_{2}$ são chamadas algumas vezes na literatura de desmembramentos.

${ }^{10}$ De acordo com a nomenclatura que temos adotado até aqui, talvez fosse mais adequado chamar essas Bases de Gröbner de "Bases de Gröbner racionais". Nào o faremos por dois motivos. Primeiro, pois assim mantemos a nomenclatura original dada por Mansson e Nordbeck [MN2]. Além disso, o termo "Base de Gröbner racional" já foi utilizado por Mansson para se referir a outro conceito [Man01].
} 
Definição 5.4.1 (Base de Gröbner Regular) Consideremos a álgebra livre $K\langle X\rangle$, com uma ordem admissivel $\leq$ fixada na base $X^{*}$ e o monóide produto $M:=X^{*} \times X^{*}$. Seja I um ideal de $K\langle X\rangle$ e $G$ uma Base de Gröbner para $I$ (com respeito à base $X^{*}$ e à ordem $\leq$ ) composta somente por binômios puros. Dizemos que $G$ é uma Base de Gröbner regular para $I$ (com respeito à ordem $\leq$ ) se existe uma relação racional $R$ de $M$, satisfazendo:

1. $f(R)=G e$

2. $f_{1}(R)=\widehat{G}$.

Definição 5.4.2 (Álgebra Bi-Autômato) Uma apresentação de uma álgebra da forma $A=$ $K\langle X\rangle / I$ é dita uma apresentação bi-autômato se existe alguma ordem admissivel (em $X^{*}$ ) $\leq$, de modo que I admite uma Base de Gröbner regular (com respeito a $\leq$ ).

Chamaremos uma álgebra de álgebra bi-autômato se ela possuir alguma apresentação biautômato.

O Exemplo 5.4.1 ilustra a definição de Base de Gröbner regular dada acima. E válido ressaltar que, embora o exemplo possa sugerir isso, a definição não exige de forma alguma que o ideal seja homogêneo.

Exemplo 5.4.2 Tomemos o ideal de $K\langle a, b, x\rangle$ gerado por

$$
H:=\left\{a x^{2 k} b-a x^{k} \mid k \geq 1\right\}
$$

Por meio do Teorema 4.2.31, não é difícil verificar que $H$ é uma Base de Gröbner (em qualquer ordem admissível). Então, $H$ pode ser representada pela relação racional dada por

$$
R=(a, a)\left(\left(x^{2}, x\right)\right)^{*}(b, 1)
$$

Antes de seguirmos adiante explorando algumas propriedades das Bases de Gröbner regulares e das álgebras bi-autômato, nos voltamos para discutir alguns pontos envolvendo sua definição. Comentamos que a definição original de Base de Gröbner regular dada por Mansson e Nordbeck difere da aqui apresentada. Apresentaremos então a definição original dessas bases fornecida por seus criadores (Definição 3 de [MN02]) e mostraremos que existe uma certa equivalência entre ela e a Definição j.t.1.

Denotamos por

$$
\mathcal{X}:=(X \times\{1\}) \cup(\{1\} \times X)
$$

(o elemento 1 utilizado acima deve ser visto como a unidade de $X^{*}$ ) e consideramos o monóide livre gerado por $\mathcal{X}, \mathcal{X}^{*}$. Seja ainda $M=X^{*} \times X^{*}$ o monóide produto. Então definimos a aplicação

$$
\varphi: \mathcal{X}^{*} \longrightarrow K\langle X\rangle
$$

por: 


$$
\varphi\left(\left(x_{1}, y_{1}\right)\left(x_{2}, y_{2}\right) \cdots\left(x_{n}, y_{n}\right)\right):=x_{1} x_{2} \cdots x_{n}-y_{1} y_{2} \cdots y_{n}
$$

e os seguintes homomorfismos de monóides:

$$
\varphi_{1}: \mathcal{X}^{*} \longrightarrow X^{*} \quad \text { e } \quad \varphi_{2}: \mathcal{X}^{*} \longrightarrow X^{*}
$$

por:

$$
\varphi_{1}\left(\left(x_{1}, y_{1}\right)\left(x_{2}, y_{2}\right) \cdots\left(x_{n}, y_{n}\right)\right):=x_{1} x_{2} \cdots x_{n} \quad \text { e } \quad \varphi_{2}\left(\left(x_{1}, y_{1}\right)\left(x_{2}, y_{2}\right) \cdots\left(x_{n}, y_{n}\right)\right):=y_{1} y_{2} \cdots y_{n}
$$

A definição original de Mansson e Nordbeck diz que uma Base de Gröbner $G$ para um ideal $I$ de $K\langle X\rangle$ (composta somente por binômios puros) é uma Base de Gröbner regular quando existe uma linguagem racional $L$ sobre $\mathcal{X}$, satisfazendo:

1. $\varphi(L)=G$.

2. $\varphi_{1}(L)=\widehat{G}$.

A fim de ilustrar essa nova definição, retomamos os Exemplos 5.4.1 e 5.4.2.

Exemplo 5.4.3 Se retomarmos o conjunto $G$ definido no Exemplo 5.4.1, podemos representá-lo pela seguinte linguagem racional sobre $\mathcal{X}$ :

$$
L=(x, 1)(1, x)((1, y)(y, 1))^{*}(x, 1)(1, y)
$$

É imediato ver que $\varphi(L)=G$ e $\varphi_{1}(L)=\widehat{G}$. Note, entretanto que se definirmos a linguagem regular $L^{\prime}$ sobre $\mathcal{X}$ como

$$
L^{\prime}=(1, x)(x, 1)((1, y)(y, 1))^{*}(x, 1)(1, y)
$$

as imagens de $L^{\prime}$ pelas aplicações $\varphi$ e $\varphi_{1}$ também são $G$ e $\widehat{G}$, respectivamente.

Ainda, se tomarmos o conjunto $H$ definido no Exemplo 5.4.2, vemos que $H$ pode ser representado pela seguinte linguagem racional:

$$
A=(a, 1)(1, a)((x, 1)(x, 1)(1, x)))^{*}(b, 1)
$$

Nosso objetivo é mostrar que as duas definições de Base de Gröbner regular possuem a mesma "força". Mais precisamente, provaremos a seguinte proposição.

Proposição 5.4.1 Seja $G$ em $K\langle X\rangle$ uma Base de Gröbner composta somente por binômios puros. Então, as seguintes condições são equivalentes:

1. Existe uma linguagem racional $L$ sobre $\mathcal{X}$, tal que $\varphi(L)=G$ e $\varphi_{1}(L)=\widehat{G}$. 
2. Existe uma relação racional $R$ em $M$ tal que $f(R)=G$ e $f_{1}(R)=\widehat{G}$.

Antes de apresentar a demonstração dessa proposição, precisaremos de uma definição e de alguns resultados.

Assim, definimos a aplicação:

$$
\begin{aligned}
\alpha: & \mathcal{X} \longrightarrow M \\
& (x, y) \mapsto(x, y)
\end{aligned}
$$

Denotaremos também por $\alpha$ sua única extensão para um homomorfismo de monóides de $\mathcal{X}^{*}$ em $M$.

Lema 5.4.2 A aplicação $\alpha$ é um homomorfismo sobrejetor de monóides.

Demonstração. É claro que $\alpha$ é um homomorfismo de monóides. Vejamos que é sobrejetor.

Seja então $(x, y)$ em $M$. Então $x$ e $y$ são palavras sobre $X$. Logo, $x=x_{1} x_{2} \cdots x_{n}$ e $y=y_{1} y_{2} \cdots y_{k}, \operatorname{com} x_{i}$ e $y_{j}$ em $X$, para todos $1 \leq i \leq n$ e $1 \leq j \leq k$.

Logo,

$$
\alpha\left(\left(x_{1}, 1\right)\left(x_{2}, 1\right) \cdots\left(x_{n}, 1\right)\left(1, y_{1}\right)\left(1, y_{2}\right) \cdots\left(1, y_{k}\right)\right)=(x, y)
$$

o que encerra a demonstração.

A demonstração da seguinte afirmação é bastante natural e deixada a cargo do leitor.

Afirmação 5.4.3 As seguintes igualdades são verdadeiras:

$$
f \alpha=\varphi \quad e \quad f_{1} \alpha=\varphi_{1}
$$

Em outras palavras, a afirmação acima nos mostra que os seguintes diagramas comutam:
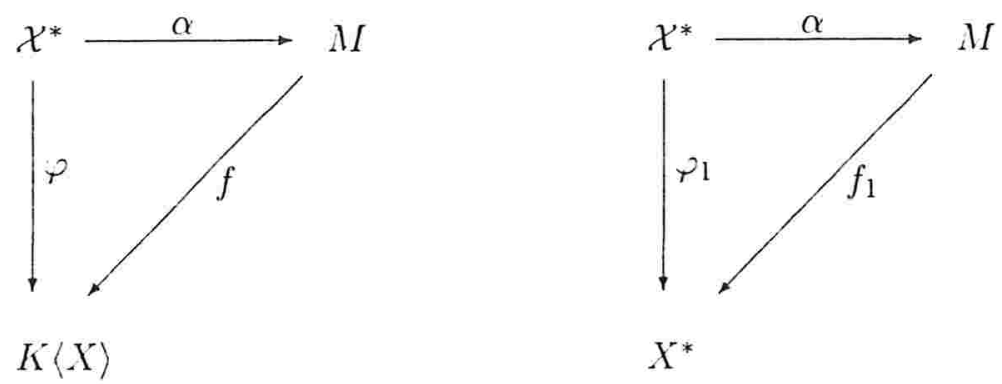

Com isso, acabamos de estabelecer a demonstração da Proposição 5̆.4.1. 


\section{Demonstração da Proposição 5.4.1.}

A equivalência das duas condições segue sem muita dificuldade da Proposição 5.3.1, do Lema 5.4.2 e da Afirmação 5.4.3.

Podemos fazer a seguinte observação acerca das funções definidas até aqui.

Observação 5.4.4 Seja $X$ um alfabeto e $H$ o conjunto composto por todos os binômios puros de $K\langle X\rangle$. Então, $\operatorname{Im}(f)=H$ e a função $f$ é injetora. Ademais, $\operatorname{Im}(\varphi)=H$, mas $\varphi$ não é necessariamente injetora.

Demonstração. As igualdades $\operatorname{Im}(f)=H=\operatorname{Im}(\varphi)$ e o fato de que $f$ é injetora são de verificação simples.

Para ver que $\varphi$ não é necessariamente injetora, basta recorrer ao Exemplo 5.4.3.

Vale ressaltar que, em seu artigo original, Mansson e Nordbeck comentam sobre a possibilidade de definir as Bases de Gröbner regulares de uma maneira alternativa, definido o alfabeto $\mathcal{X}$ como $\mathcal{X}=(X \cup\{1\}) \times(X \cup\{1\})$. Devemos ressaltar que essa definição ainda é ligeiramente diferente da dada por nós, na Definição 5.4.1.

Justificamos nossa preferência pela forma apresentada na Definição 5.4 .1 pelo fato de que as relações racionais, apesar de serem objetos "mais abstratos" que palavras sobre $\mathcal{X}$ (e constituírem um caso mais geral que elas) parecem refletir melhor os conjuntos de binômios puros em que estamos interessados. De fato, a Observação 5.4 .4 nos mostra que podemos identificar conjuntos de binômios puros com relações no monóide produto $M$ e que o mesmo não pode ser feito com linguagens em $\mathcal{X}^{*}$.

Dessa forma, parece-nos que as linguagens sobre $\mathcal{X}$ formam representantes para um conjunto de binômios puros, enquanto as relações em $M$ capturam melhor suas propriedades. Assim, um conjunto de binômios puros é representado por um conjunto de linguagens sobre $\mathcal{X}$ (não necessariamente racional), mas apenas por uma relação sobre $M$ (não necessariamente racional).

Ainda, dependendo da pergunta a ser respondida, diferentes representantes (em $\mathcal{X}^{*}$ ) para um mesmo conjunto de binômios puros, podem fornecer diferentes respostas. Ilustramos esse comentário através do exemplo a seguir.

Exemplo 5.4.4 Seja $X=\{a, x, y\}$ e consideremos o conjunto $G=\{x y-a\} \in K\langle X\rangle$. Consideramos o problema de representar $G$ por um $\mathcal{X}$-autômato e, em seguida, utilizá-lo para decidir a pertinência de um elemento qualquer em $G$.

Consideremos então o seguinte autômato $\mathcal{A}$ :

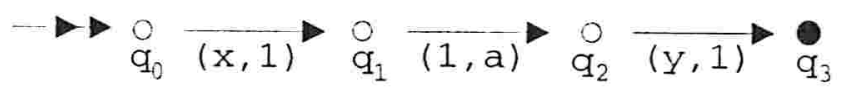

É imediato ver que $\varphi(|\mathcal{A}|)=G$. Consideramos então o problema de decidir se $x y-a$ pertence a $G$. Para tanto, consideramos as seguintes palavras sobre $\mathcal{X}$ :

$$
w:=(x, 1)(y, 1)(1, a) \text { e } w^{\prime}:=(x, 1)(1, a)(y, 1)
$$

É claro que $\varphi(w)=\varphi\left(w^{\prime}\right)=x y-a$. Contudo, $w \notin|\mathcal{A}|$, mas $w^{\prime} \in|\mathcal{A}|$.

Por outro lado, se considerarmos o transdutor $\mathcal{M}$, dado por: 


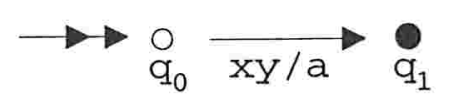

vemos que $(x, 1)(y, 1)(1, a)=(x, 1)(1, a)(y, 1)=(x y, a) \in|\mathcal{M}|$.

Finalmente, comentamos que existem algumas perguntas importantes envolvendo Bases de Gröbner regulares que não foram respondidas (veja Seção 5.6). Podemos citar um problema central que consiste em decidir se um ideal (dado por geradores) possui alguma Base de Gröbner regular (para a ordem admissível fixada).

Assim, temos a intenção de formar um bom arsenal de propriedades ao lidarmos com a definição de Base de Gröbner regular, a fim de ter à disposição mais recursos na tentativa de resolução desses problemas.

Diante disso, podemos comentar que as relações racionais foram vastamente estudadas e muitas de suas propriedades são conhecidas. Assim, temos a esperança de que esse maquinário seja de alguma ajuda na resolução desses problemas. Ademais, a Proposição 5.4.1 nos mostra que podemos utilizar as duas formas de ver Bases de Gröbner regulares na busca por ideais que admitam tais bases.

Encerramos esta discussão mencionando que o Corolário 6.2 do livro de Berstel [Ber79] nos mostra que, na realidade, toda relação racional pode ser descrita por um $\mathcal{X}$-autômato. ${ }^{11}$ Isso corrobora nossa observação anterior de que as relações racionais capturam melhor a natureza dos conjuntos de binômios puros do que linguagens sobre $\mathcal{X}$.

Seguimos apresentando alguns resultados envolvendo Bases de Gröbner regulares.

Proposição 5.4.5 Seja $A=K\langle X\rangle / I$ uma apresentação bi-autômato. Então $K\langle X\rangle / I$ é uma apresentação de autômato.

Demonstração. Da definição de apresentação bi-autômato, vemos que existe uma Base de Gröbner regular $G$ para $I$. Mas então, como $f_{1}$ é um homomorfismo segue que $\widehat{G}$ é uma linguagem racional sobre $X$. O resultado segue então do Corolário 5.2.2.

Proposição 5.4.6 Se G é uma Base de Gröbner regular para um ideal I de $K\langle X\rangle$, então existe uma Base de Gröbner minimal e regular para $I$.

Demonstração. Inicialmente, lembramos que pela discussão ao final da Seção 5.1, $O_{I}=\operatorname{red}(\widehat{G})$ e o conjunto

$$
G^{\prime}:=\left\{g \in G \mid \widehat{g} \in O_{I}\right\}
$$

é uma Base de Gröbner minimal para $I$.

Como $G$ é uma Base de Gröbner regular para $I$, existe uma relação racional $R$ sobre o monóide produto $M=X^{*} \times X^{*}$, de modo que $f(R)=G$ e $f_{1}(R)=\widehat{G}$. Ainda, como $f_{1}$ é um homomorfismo de monóides, segue que $\widehat{G}$ é uma linguagem racional sobre $X$. Assim, $O_{I}=\operatorname{red}(\widehat{G})$ é uma linguagem racional sobre $X$, pelo Lema 5.1.7.

\footnotetext{
${ }^{11}$ De fato, esse resultado poderia ter sido utilizado na demonstração da Proposição 5.4.1. Contudo, preferimos a versão aqui fornecida por ser mais algébrica.
} 
Seja então $\mathcal{A}=(Q, M, S, T, E)$ um $M$-autômato que reconhece $R$ e $\mathcal{B}=$ $\left(Q^{\prime}, X,\{i\}, T^{\prime}, \alpha\right)$ um $X$-autômato que reconhece $O_{I}$ (podemos assumir que $\mathcal{B}$ é determinístico). Construiremos um $M$-autômato $\mathcal{M}$ que reconhece $G^{\prime}$.

Definimos então $\mathcal{M}=\left(Q \times Q^{\prime}, M, S \times\{i\}, T \times T^{\prime}, E^{\prime}\right)$, com o conjunto de transições $E^{\prime}$ definido da seguinte forma:

Uma transição $\left(\left(q_{1}, q_{1}^{\prime}\right),\left(u, u^{\prime}\right),\left(q_{2}, q_{2}^{\prime}\right)\right)$ está em $\mathcal{M}$ se e somente se:

1. Existe uma transição $\left(q_{1},\left(u, u^{\prime}\right), q_{2}\right)$ em $\mathcal{A}$ e

2. Existe um caminho em $\mathcal{B}$ de $q_{1}^{\prime}$ a $q_{2}^{\prime}$ com rótulo $u .^{12}$

A finitude de $E^{\prime}$ segue imediatamente do fato de $E$ e $Q^{\prime}$ serem finitos.

Com isso, não é difícil ver que existe um caminho de $\left(q_{1}, q_{1}^{\prime}\right)$ a $\left(q_{2}, q_{2}^{\prime}\right)$ com rótulo $u$ se e somente se existem:

1. Um caminho de $q_{1}$ a $q_{2}$ com rótulo $\left(u, u^{\prime}\right)$ em $\mathcal{A}$.

2. Um caminho de $q_{1}^{\prime}$ a $q_{2}^{\prime}$ com rótulo $u$ em $\mathcal{B}$.

Assim, $\left(u, u^{\prime}\right)$ pertence a $|\mathcal{M}|$ se e somente se $\left(u, u^{\prime}\right)$ pertence a $|\mathcal{A}|$ e $u$ pertence a $|\mathcal{B}|$. Logo, $\left(u, u^{\prime}\right)$ pertence a $|\mathcal{M}|$ se e somente se $u-u^{\prime}$ pertence a $G$ e $u$ pertence a $O_{I}$, para todo $\left(u, u^{\prime}\right)$ em $M$. Portanto, concluímos que $f(|\mathcal{M}|)=G^{\prime}$ e $f_{1}(|\mathcal{M}|)=\widehat{G^{\prime}}=O_{I}$, o que encerra a demonstração.

Uma pequena modificação na demonstração da proposição acima nos permite concluir o seguinte corolário.

Corolário 5.4.7 Se G é uma Base de Gröbner regular para um ideal I de $K\langle X\rangle$, de modo que $G$ contém a Base de Gröbner reduzida $G_{\mathrm{Red}}$ para $I$, então $G_{\mathrm{Red}}$ é uma Base de Gröbner regular.

Demonstração. Seja $G$ uma Base de Gröbner regular para $I$. Pela Proposição 5.4.6, podemos assumir que $G$ é minimal. Notamos primeiro que

$$
\operatorname{Norm}(\mathrm{I})=X^{*} \backslash X^{*}(\widehat{G}) X^{*}
$$

Desse modo, pela Proposição 1.j.1, Norm(I) será uma linguagem racional sobre $X$, se $\widehat{G}$ o for.

Mas, como $G$ é uma Base de Gröbner regular, existe uma relação racional $R$, de modo que $f_{1}(R)=\widehat{G}$. A racionalidade de $\widehat{G}$ segue então da Proposição 5.3.1.

Agora, como $G_{\text {Red }}$ está contido em $G$, não é difícil ver a partir da definição de Base de Gröbner reduzida e do Corolário 3.1.13 que

$$
G_{\text {Red }}=\left\{a-b \in G \mid a, b \in X^{*} \text { e } b \in \operatorname{Norm}(\mathrm{I})\right\}
$$

\footnotetext{
${ }^{12}$ Note que, como assumimos que $\mathcal{B}$ é deterministico, para todo par $\left(q_{1}^{\prime}, u\right)$ de $Q^{\prime} \times X^{*}$, existe exatamente um caminho com origem em $q_{1}^{\prime}$ e rótulo $u$.
} 
Seja então $\mathcal{A}=(Q, M, S, T, E)$ um $M$-autômato que reconhece $R$ e $\mathcal{B}=$ $\left(Q^{\prime}, X,\{i\}, T^{\prime}, \alpha\right)$ um $X$-autômato que reconhece Norm(I) (novamente, podemos assumir que $\mathcal{B}$ é determinístico). Então basta construir um $M$-autômato $\mathcal{M}$ que reconhece $G_{\text {Red }}$, de maneira totalmente análoga ao que foi feito na demonstração da Proposição 5.4.6.

Por outro lado, o exemplo a seguir mostra que a implicação descrita no Corolário 5.4 .7 não é válida se retirarmos a hipótese de que $G_{\text {Red }}$ está contida em $G$ (ao menos para ideais infinitamente gerados).

Exemplo 5.4.5 Tomemos o alfabeto $X=\{x, y, a, b\}$ e consideremos a álgebra $K\langle X\rangle$ com a ordem de grau e lexicografia fixada em $X^{*}$ (com a seguinte ordem nas letras: $x>y>a>b$ ). Seja $G$ o conjunto definido por

$$
G:=\left\{a x^{2 i} b-(x y)^{i}, x y-y x \mid i \geq 1\right\}
$$

e $I$ o ideal (infinitamente) gerado por $G$.

Não é difícil ver que não existem relações de divisão nem de sobreposição envolvendo elementos de $G$, de modo que $G$ é uma Base de Gröbner para $I$. Ademais, $G$ é uma Base de Gröbner minimal (por não existirem relações de divisão e pelo Corolário 3.2.10).

Além disso, existe uma relação racional $R$ sobre o monóide produto $M:=X^{*} \times X^{*}$ que "codifica" G:

$$
R:=(a, 1)\left(x^{2}, x y\right)^{+}(b, 1) \cup(x y, y x)
$$

É fácil ver que $f(R)=G$ e $f_{1}(R)=\widehat{G}$.

Vejamos agora quem é a Base de Gröbner reduzida de $I$. Mas, como $G$ é uma Base de Gröbner minimal, pelo Corolário 3.2.14, basta encontrar a forma normal de cada elemento de $\widehat{G}$. Alguns cálculos rotineiros nos mostram que:

$$
\begin{aligned}
& N(x y)=y x \mathrm{e} \\
& N\left(a x^{2 i} b\right)=y^{i} x^{i}, \text { para todo } i \geq 1 .
\end{aligned}
$$

Portanto, a Base de Gröbner reduzida para $I$ é

$$
G_{\text {Red }}=\left\{a x^{2 i} b-y^{i} x^{i}, x y-y x \mid i \geq 1\right\}
$$

Vejamos que não existe nenhuma relação racional $S$ sobre o monóide produto $M$ que satisfaz $f(S)=G_{\text {Red }}$ e $f_{1}(S)=\widehat{G_{\text {Red }}}$. A demonstração desse fato é simples segue por redução ao absurdo.

Suponha que $S$ é uma relação racional satisfazendo as condições acima. Mas então, $f_{2}(S)$ é uma linguagem racional sobre $X$, pela Proposição 5.3.1. Porém, 


$$
f_{2}(S)=\left\{y^{i} x^{i} \mid i \geq 1\right\}
$$

nos levando a uma contradição com o Lema da Iteração (Proposição 1.5.5).

O corolário abaixo é conseqüência da Proposição 5.4.6.

Corolário 5.4.8 dada uma ordem admissivel $\leq$ em $X^{*}$, se I é um ideal de $K\langle X\rangle$ e $O_{I}$ não é uma linguagem racional sobre $X$, então I não possui Base de Gröbner regular (com respeito à ordem $\leq$ ).

\subsection{Boas Representações e Reduções}

Conforme comentado no início do capítulo, o principal interesse no estudo das Bases de Gröbner regulares está no fato de que elas fornecem boas representações de Bases de Gröbner (Definição 4.4.1). Com isso, essas boas representações nos trazem a possibilidade de efetuar reduções sobre uma Base de Gröbner infinita, utilizando o Algoritmo da Divisão.

Para tanto, de acordo com as discussões vistas na Seção 4.4, basta termos uma representação finita para uma Base de Gröbner $G$ e um algoritmo que receba como entrada um monômio $m$ e devolva um elemento $g$ de $G$ tal que $\widehat{g}$ divide $m$ (no caso de tal elemento $g$ não existir, o algoritmo deve fornecer a garantia de que ele não existe). Nesta seção, tecemos alguns breves comentários sobre como uma versão ligeiramente modificada desse algoritmo pode ser dada no contexto das Bases de Gröbner regulares.

Assumimos então que temos uma Base de Gröbner regular $G$ de um ideal $I$ de $K\langle X\rangle$ e um transdutor $\mathcal{T}=(Q, M, S, T, E)$, de modo que $f(|\mathcal{T}|)=G$ e $f_{1}(|\mathcal{T}|)=\widehat{G}$. Nosso objetivo é ter uma relação racional $R$ de maneira que, para todo monômio $m, R(m)$ possui as seguintes propriedades:

- A imagem de $m$ por $R$ é vazia se e somente se não existe $g$ em $G$ com $\widehat{g}$ dividindo $m$.

- Um elemento $h$ pertence a $R(m)$ se e somente se existem $a$ e $b$ em $X^{*}$ e $g=t-t^{\prime}$ em $G$, de modo que $\widehat{g}=t, a t b=m$ e $a t^{\prime} b=h$.

É claro que se conseguimos realizar uma relação $R$ com as propriedades descritas acima, então conseguimos computar efetivamente reduções simples de elementos de $K\langle X\rangle$ por elementos de $G$. Isso é feito da seguinte forma: se $f$ é um elemento de $K\langle X\rangle$ e $h \in R(f)$, fazemos:

$$
f^{\prime}=f-\operatorname{cl}(f) \hat{f}+\operatorname{cl}(f) h
$$

Vejamos então como construir um transdutor $\mathcal{T}^{\prime}$ que realize a relação racional $R$ com as propriedades descritas acima. ${ }^{13}$ Lma vez que tivermos $\mathrm{um}$ tal transdutor, existem algoritmos que possibilitam obter a imagem de uma palavra por $R$ [Lot03].

Pela Proposição 5.3.4, podemos assumir que $\mathcal{T}$ é normalizado. Assim, $S=\{i\}$ e $T=\{t\}$. Seja então $\mathcal{T}^{\prime}=\left(Q, M,\{i\},\{t\} . E^{\prime}\right)$, com $E^{\prime}$ dado por:

$$
E^{\prime}=E \cup\{(q,(x, x), q) \mid q \in\{i, t\} \text { e } x \in X\}
$$

Vejamos que o transdutor $\mathcal{T}^{\prime}$ atende aos nossos propósitos.

\footnotetext{
${ }^{13}$ A sugestão da construção a seguir foi dada por A. Mandel (comunicação pessoal).
} 
Proposição 5.5.1 Seja $m$ um monômio em $X^{*}$. Então $\left|\mathcal{T}^{\prime}\right|(m)=\emptyset$ se e somente se não existe $g$ em $G$ com $\widehat{g}$ dividindo $m$. Ademais, $h$ pertence a $R(m)$ se e somente se existem a e $b$ em $X^{*} e$ $g=t-t^{\prime}$ em $G$, de modo que $\widehat{g}=t$, atb $=m$ e $a t^{\prime} b=h$.

Demonstração. Pela construção de $\mathcal{T}^{\prime}$, é imediato ver que se existe um caminho bem sucedido em $\mathcal{T}$ soletrando $\left(u, u^{\prime}\right) \in M$, então existem caminhos bem sucedidos em $\mathcal{T}^{\prime}$, soletrando $\left(a u b, a u^{\prime} b\right) \in M$, para todos $a$ e $b$ em $X^{*}$. Inversamente, se $\left(w, w^{\prime}\right)$ é o rótulo de um caminho bem-sucedido em $\mathcal{T}^{\prime}$, existem $u, u^{\prime}$, $a$ e $b$ em $X^{*}$, de modo que $a u b=w$ e $a u^{\prime} b=w^{\prime}$ e $\left(u, u^{\prime}\right) \in|\mathcal{T}|$.

Com isso, $\left(u, u^{\prime}\right)$ pertence a $\left|\mathcal{T}^{\prime}\right|$ se e somente se existem $g=t-t^{\prime}$ em $G(\widehat{g}=t)$ e $a$ e $b$ em $X^{*} \operatorname{com} a t b=u$ e $a t^{\prime} b=u^{\prime}$.

$\mathrm{O}$ resultado segue então por um argumento bem natural.

Encerramos esta seção com o comentário de que a construção dada acima não fornece uma boa representação de Base de Gröbner no sentido exato da Definição 4.4.1 (apesar de ela resolver o problema de efetuar reduções sobre o conjunto infinito $G$ ). Por outro lado, é bastante simples utilizar as ferramentas desenvolvidas até aqui para exibir explicitamente uma boa representação de Base de Gröbner para uma Base de Gröbner regular $G$.

Primeiro, note que $\widehat{G}$ é uma linguagem regular sobre $X$ (por ser a imagem de $G$ por $f_{1}$ ). Assim, podemos assumir que temos um $X$-autômato $\mathcal{A}$ que reconhece $\widehat{G}$. De fato, $\mathcal{A}$ pode ser construído a partir de $\mathcal{T}$ da seguinte forma: primeiro substituímos o rótulo $(u, v)$ de cada transição de $\mathcal{T}$ por $u$. Em seguida, substituímos cada transição rotulada por um elemento $u=x_{1} x_{2} \cdots x_{n}$ (com $x_{i}$ em $X$, para cada $1 \leq i \leq n$ ) por $n$ transições rotuladas pelas letras que compõem $u$ (inserindo estados conforme necessário). O único cuidado que resta é que $\mathcal{A}$ pode possuir transições rotuladas pela palavra vazia. Entretanto, é bem conhecido o fato de que um autômato desse tipo é equivalente ao conceito apresentado na Definição 1.5.2 [HU79].

Com isso, dado um monômio $m$ de $X^{*}$, temos em mãos o famoso problema de encontrar divisores de $m$ que estejam em $|\mathcal{A}|$. Uma vez encontrado um divisor $h$ de $m$, basta encontrar $h^{\prime}$ em $|\mathcal{T}|(h)$. $h-h^{\prime}$ será então um elemento de $G$ tal que $h$ divide $m$.

\subsection{Considerações Finais}

Antes de encerrar o capítulo, apresentamos algumas considerações finais e observações acerca de Bases de Gröbner regulares.

A primeira e mais importante observação se refere a sua construção. Seria ideal possuir um algoritmo que receba como entrada um conjunto (finito) puramente binomial de geradores para um ideal $I$ da álgebra livre e, ou devolva um transdutor que represente uma Base de Gröbner regular para $I$, ou forneça a garantia de que tal ideal não possui nenhuma Base de Gröbner regular (ao menos para a ordem admissivel fixada em $X^{*}$ ).

Infelizmente, não se sabe sequer se o problema de decidir se um ideal (puramente binomial) $I$ de $K\langle X\rangle$ (dado por geradores) possui alguma Base de Gröbner regular é decidível.

O seguinte problema foi sugerido por A. Mandel como um primeiro ponto a ser resolvido antes de considerar os problemas acima: dado um transdutor que representa um conjunto $F$ de binômios puros de $K\langle X\rangle$, decida se $F$ é uma Base de Gröbner. Infelizmente, os autores ainda não sabem 
se esse problema é decidível e nenhuma resposta para ele é fornecida na literatura (até onde é de conhecimento dos autores).

Comentamos ainda que uma tentativa de "prever" uma Base de Gröbner regular $G$ para um ideal $I$ de $K\langle X\rangle$ a partir de um conjunto inicial finito de elementos de $G$ foi apresentada por Mansson [Man00]. ${ }^{14}$ Em seu artigo, Mansson fornece um algoritmo que recebe como entrada um conjunto finito de palavras $S$ (sobre $\mathcal{X}$ ), de comprimento limitado por um inteiro positivo $k$, representando um conjunto de binômios puros e devolve todas as linguagens racionais $S_{i}$, satisfazendo:

1. O conjunto de todas as palavras de $S_{i}$ de comprimento no máximo $k$ é exatamente $S$.

2. Cada linguagem $S_{i}$ é reconhecida por um autômato cujo número de estados é minimal entre os autômatos que aceitam linguagens que satisfazem a primeira condição.

Esse algoritmo pode ser usado como uma ajuda para encontrar Bases de Gröbner infinitas (desde que eles sejam regulares). Contudo, ele possui o sério problema de não fornecer um certificado para sua saída. De fato, os elementos das linguagens $S_{i}$ devolvidas pelo algoritmo podem "não pertencer a $I^{\prime}$ ou, mesmo que pertençam, o conjunto representado por uma essas linguagens pode não ser um Base de Gröbner. Conforme comentado em [Man00], não se sabe se o problema de decidir se $S_{i}$ é uma Base de Gröbner para $I$ é decidível.

Vale mencionar que uma generalização do conceito de Base de Gröbner regular foi apresentada por Mansson [Man01]. Trata-se das Bases de Gröbner racionais, que têm a intenção de representar por um tipo de transdutor (suas emissões são séries formais de potências) Bases de Gröbner compostas não somente por binômios puros.

\footnotetext{
${ }^{14}$ Esse conjunto inicial finito de elementos de $G$ pode ser obtido, por exemplo, por alguma computação inicial do Procedimento de Mora.
} 


\section{REFERÊNCIAS BIBLIOGRÁFICAS}

[AL94] W. Adams and P. Loustaunau, An introduction to Gröbner bases, Graduate Studies in Mathematics, vol. 3, American Mathematical Society, 1994. Citado na(s) página(s) 26, 34, 43

[Ani86] D. J. Anick, On the homology of associative algebras, Trans. AMS 296 (1986), 641-659. Citado na(s) página(s) 180

[ARS95] M. Auslander, I. Reiten, and S. O. Smalo, Representation theory of artin algebras, Cambridge University Press, 1995. Citado na(s) página(s) 14

[Ber78] G. M. Bergman, The diamond lemma for ring theory, Adv. in Math. 29 (1978), no. 2, 178-218. Citado na(s) página(s) v, vii, 26, 45, 115, 137

[Ber79] J. Berstel, Transductions and context-free languages, Teubner, 1979. Citado na(s) página(s) 12, $16,183,184,195$

[BGRS85] R. Bautista, P. Gabriel, A. V. Roiter, and L. Salmerón, Representation-finite algebras and multiplicative bases, Invent. Math. 81 (1985), no. 2, 217-285. Citado na(s) página(s) 47

[BM76] J. A. Bondy and U. S. R. Murty, Graph theory with applications, Macmillan/Elsevier, 1976. Citado na(s) página(s) 12

[Bün91] R. Bündgen, Simulating Buchberger's algorithm by Knuth-Bendix completion, RTA '91 (R. V. Book, ed.), Lecture Notes in Computer Science, vol. 488, Springer-Verlag, 1991, pp. 386-397. Citado na(s) página(s) 45

[Bün92] _ Buchberger's algorithm: the term rewriter point of view, ICALP '92 (W. Kuich, ed.), Lecture Notes in Computer Science, vol. 623, Springer-Verlag, 1992, pp. 380-391. Citado na(s) página(s) 45

[BO93] R. V. Book and F. Otto, String-rewriting systems, Springer-Verlag, 1993. Citado na(s) página(s) 45

[Boki6] L. A. Bokut, Imbeddings into simple associative algebras, Algebra i Logika 15 (1976), no. 2, 117-142, 245. Citado na(s) página(s) v, 26, 45

[Buc65] B. Buchberger, On finding a vector space basis of the residue class ring modulo a zero dimensional polynomial ideal, Ph.D. thesis, Univ. of Innsbruck, Austria, 1965. Citado na(s) página(s) v, 25, 34,42

[Buci9] _ A criterion for detecting unnecessary reductions in the construction of Gröbner bases, ELROSAM 79: Proceedings of the International Symposiumon on Symbolic and Algebraic Computation, Springer- Verlag, 1979, pp. 3-21. Citado na(s) página(s) 43 
[BW93] T. Becker and V. Weispfenning, Gröbner bases: a computational approach to commutative algebra, Springer-Verlag, 1993, In cooperation with Heinz Kredel. Citado na(s) página(s) 1, $9,26,34,42,43$

[CLO97] D. Cox, J. Little, and D. O'Shea, Ideals, varieties, and algorithms : an introduction to computational algebraic geometry and commutative algebra, 2nd ed., Springer, 1997. Citado na(s) página(s) 26, 33, 34, 42, 43

[CLRS01] T. H. Cormen, C. E. Leiserson, R. L. Rivest, and C. Stein, Introduction to algorithms, 2nd ed., McGraw-Hill, 2001. Citado na(s) página(s) 12, 22

[CLS83] C. Cibils, F. Larrión, and L. Salmerón, Métodos diagramáticos en teoria de representaciones, Inst. de Matemática de la UNAM, México, 1983. Citado na(s) página(s) 9, 14, 15

[DJ90] N. Dershowitz and J. P. Jouannaud, Rewrite systems, Handbook of Theoretical Computer Science B: Formal Methods and Semantics (J. van Leeuwen, ed.), vol. B, North-Holland, Amsterdam, 1990, pp. 243-320. Citado na(s) página(s) 45

[dS04] R. N. P. M. de Souza, Propriedades de algumas classes de relações racionais, Master's thesis, IME-USP, 2004. Citado na(s) página(s) 183

[Eil74] S. Eilenberg, Automata, languages, and machines, vol. A, Pure and applied mathematics (Academic Press), no. 59, Academic Press, 1974. Citado na(s) página(s) 183

[Feo99] P. Feofiloff, Algoritmos de programação linear, EDUSP (a ser publicado), 1999. Citado na(s) página(s) 27

[Feo01] , Notas de aula da disciplina "Algoritmos em Grafos com Stanford GraphBase", 2001, Disponivel em: http://www.ime.usp.br/ pf/algoritmos_em_grafos/. Citado na(s) página(s) 12

[Fer95] V. O. Ferreira, O lema do diamante em teoria de anéis, Master's thesis, IME-USP, 1995. Citado na(s) página(s) 115, 141

[FFG93] D. R. Farkas, C. D. Feustel, and E. L. Green, Synergy in the theories of Gröbner bases and path algebras, Can Journal of Math. 45 (1993), 727-739. Citado na(s) página(s) vi, vii, 45, 60, 73, 146

[FJ03] J. Faugère and A. Joux, Algebraic cryptanalysis of hidden field equation (HFE) cryptosystems using Gröbner bases, Advances in cryptology-CRYPTO 2003, Lecture Notes in Comput. Sci., vol. 2729, Springer, Berlin, 2003, pp. 44-60. Citado na(s) página(s) 43

[Fra99] J. B. Fraleigh, A first course in abstract algebra, 6th ed., Addison-Wesley, 1999, Historical notes by Victor Katz. Citado na(s) página(s) 5

[GCL92] K. O. Geddes, S. R. Czapor, and G. Labahn, Algorithms for computer algebra, Kluwer Academic Publishers, 1992. Citado na(s) página(s) 113

[GHK97] E. L. Green, L. S. Heath, and B. J. Keller, Opal: A system for computing noncommutative Gröbner bases, RTA-97 (H. Comon, ed.), Lecture Notes in Computer Science, vol. 1232, SpringerVerlag, 1997. Citado na(s) página(s) 46

[G.M88] R. Gebauer and H. MI. Möller, On an installation of Buchberger's algorithm, J. Symbolic Comput. 6 (1988), no. 2-3, 275-286. Citado na(s) página(s) 43

[GMN ${ }^{+91]}$ A. Giovini, T. Mora, G. Niesi, L. Robbiano, and C. Traverso, "One sugar cube, please"or selection strategies in the Buchberger algorithm, ISSAC' '91: Proceedings of the 1991 international symposium on Symbolic and algebraic computation, ACM Press, 1991, pp. 49-54. Citado na(s) página(s) 43 
[GMU98] E. L. Green, T. Mora, and V. Ufnarovski, The non-commutative Gröbner freaks, Symbolic rewriting techniques (Ascona, 1995), Progr. Comput. Sci. Appl. Logic, vol. 15, Birkhäuser, Basel, 1998, pp. 93-104. Citado na(s) página(s) 47

[Gre97] E. L. Green, Noncommutative Gröbner bases and projective resolutions, Progress in Mathematics (Basel) (Michler and Scheider, eds.), vol. 173, Euroconference Computational Methods for Representations of Groups and Algebras, Birkhäuser Verlag, 1997, pp. 29-60. Citado na(s) página(s) vi, vii, $45,115,146$

[Gre00] - Multiplicative bases, Gröbner bases and right Gröbner bases, Journal of Symbolic Computation 29 (2000), no. 4-5, 601-623. Citado na(s) página(s) 47,67, 73, 80

[Hal74] P. R. Halmos, Naive set theory, Springer-Verlag, 1974. Citado na(s) página(s) 1

[Her64] I. N. Herstein, Topics in algebra, Blaisdell Publishing Company, 1964. Citado na(s) página(s) 5

[Hey00] A. Heyworth, Rewriting as a special case of non-commutative Gröbner basis theory, Computational and Geometric Aspects of Modern Algebra, Cambridge Univ. Press, 2000, pp. 101-105. Citado na(s) página(s) 46

[Hir64] H. Hironaka, Resolution of singularities of an algebraic variety over a field of characteristic zero, Ann. Math (1964), no. 79, 109-326. Citado na(s) página(s) v, 25

[HK61] K. Hoffman and R. Kunze, Linear algebra, Prentice Hall, 1961. Citado na(s) página(s) 5, 9

[HU79] J. E. Hopcroft and J. D. Ullman, Introduction to automata theory, languages and computation, Addison-Wesley, 1979. Citado na(s) página(s) 16, 199

[Jac85] N. Jacobson, Basic algebra I, Bedford, Freeman and Worth (W H Freeman), 1985. Citado na(s) página(s) 5, 9

[KB70] D. E. Knuth and P. B. Bendix, Simple word problems in universal algebra, Computational Problems in Abstract Algebra (J. Leech, ed.), Pergamon Press, 1970, pp. 263-297. Citado na(s) página(s) 25

[Kel97] B. J. Keller, Algorithms and orders for finding noncommutative Gröbner bases, Ph.D. thesis, Virginia Polytechnic Inst. and State Univ., Blacksburg, EUA, 1997. Citado na(s) página(s) 46, 147

[Kel98] — Alternatives in implementing noncommutative Gröbner basis systems, Symbolic Rewriting Techniques (M. Bronstein, J. Grabmeier, and V. Weispfenning, eds.), BirkhauserVerlag, 1998. Citado na(s) página(s) 46, 147

[KRW90] A. Kandri-Rody and V. Weispfenning, Noncommutative Gröbner bases in algebras of solvable type, J. Symbolic Comput. 9 (1990), no. 1, 1-26. Citado na(s) página(s) vii, 47

[Lal79] G. Lallement, Semigroups and combinatorial applications, John Wiley \& Sons, 1979. Citado na(s) página(s) 173

[Lan71] S. Lang, Linear algebra, 2nd ed., Addison-Wesley, 1971. Citado na(s) página(s) 5, 9

[Lot03] M. Lothaire, Applied combinatorics on words, Cambridge Lniversity Press, 2003. Citado na(s) página(s) 198

[LRS95] F. Larrion, G. Raggi, and L. Salmeron, Rudimentos de mansedumbre y salvajismo en teoria de representaciones, Sociedad Matematica Mexicana, 1995. Citado na(s) página(s) i3

$\left[\operatorname{LSS}^{+} 79\right]$ C. L. Lucchesi, I. Simon, I. Simon, J. Simon, and T. Kowaltowski, Aspectos teóricos da computação, Instituto de Matemática Pura e Aplicada, 1979. Citado na(s) página(s) 12, 16 
[Man00] J. Mansson, A prediction algorithm for regular languages, Licentiate Thesis, Lund University, 2000. Citado na(s) página(s) 200

[Man01]

[May97] E. W. Mayr, Some complexity results for polynomial ideals, J. Complexity 13 (1997), no. 3, 303-325. Citado na(s) página(s) 43

[MN02] J. Mansson and P. Nordbeck, Regular Gröbner bases, Journal of Symbolic Computation 33 (2002), no. 2, 163-181. Citado na(s) página(s) vii, ix, 47, 66, 177, 182, 190, 191

[Mor86] F. Mora, Gröbner bases for non-commutative polynomial rings, Algebraic algorithms and errorcorrecting codes : proceedings (Jacques Calmet, ed.), Lecture Notas in Computer Science, vol. 229, Springer-Verlag, 1986, pp. 353-362. Citado na(s) página(s) v, 26, 45

[Mor91] T. Mora, Gröbner bases and the word problem, Disponível em: ftp://ftp.disi.unige.it/person/ MoraF/PUBLICATIONS/Groebner_Word.ps.gz, 1991. Citado na(s) página(s) vi, 46

[Mor94] , An introduction to commutative and noncommutative Gröbner bases, Theoretical Computer Science 134 (1994), 131-173. Citado na(s) página(s) vi, vii, viii, 26, 45, 46, 47, $107,115,146,147,172$

[Oli02] P. P. Oliveira, Bases de Gröbner, Master's thesis, IME-USP, São Paulo, Brazil, 2002. Citado na(s) página(s) 80

[Pie82] R. S. Pierce, Associative algebras, Graduate texts in mathematics, Springer-Verlag, 1982. Citado na(s) página(s) 9

[Tra89] C. Traverso, Gröbner trace algorithms, Symbolic and algebraic computation (Rome, 1988), Lecture Notes in Comput. Sci., vol. 358, Springer, Berlin, 1989, pp. 125-138. Citado na(s) página(s) 43

[Tse58] G. S. Tsejtin, Associative computations with unsolvable equivalency problems, Tr Mat. Inst. Steklova 52 (1958), 172-189. Citado na(s) página(s) 173

[Ufn80] V. Ufnarovski, Poincaré series of graded algebras, Mat. Zametki 27 (1980), no. 1, 21-32. Citado na(s) página(s) vi, 45

[Ufn89] — On the use of graphs for calculating the basis, growth and Hilbert series of associative algebras, Mathematical Sbornik 180 (1989), no. 11, 1548-1560, 1584. Citado na(s) página(s) vi, ix, 45, 181, 183

[Ufn98] , Introduction to noncommutative Gröbner bases theory, Gröbner bases and applications (Linz, 1998), London Math. Soc. Lecture Note Ser., vol. 251, Cambridge Univ. Press, Cambridge, 1998, pp. 259-280. Citado na(s) página(s) 45

[vzGG99] J. von zur Gathen and J. Gerhard, Modern computer algebra, Cambridge University Press, 1999. Citado na(s) página(s) 26, 34, 43 


\section{ÍNDICE REMISSIVO}

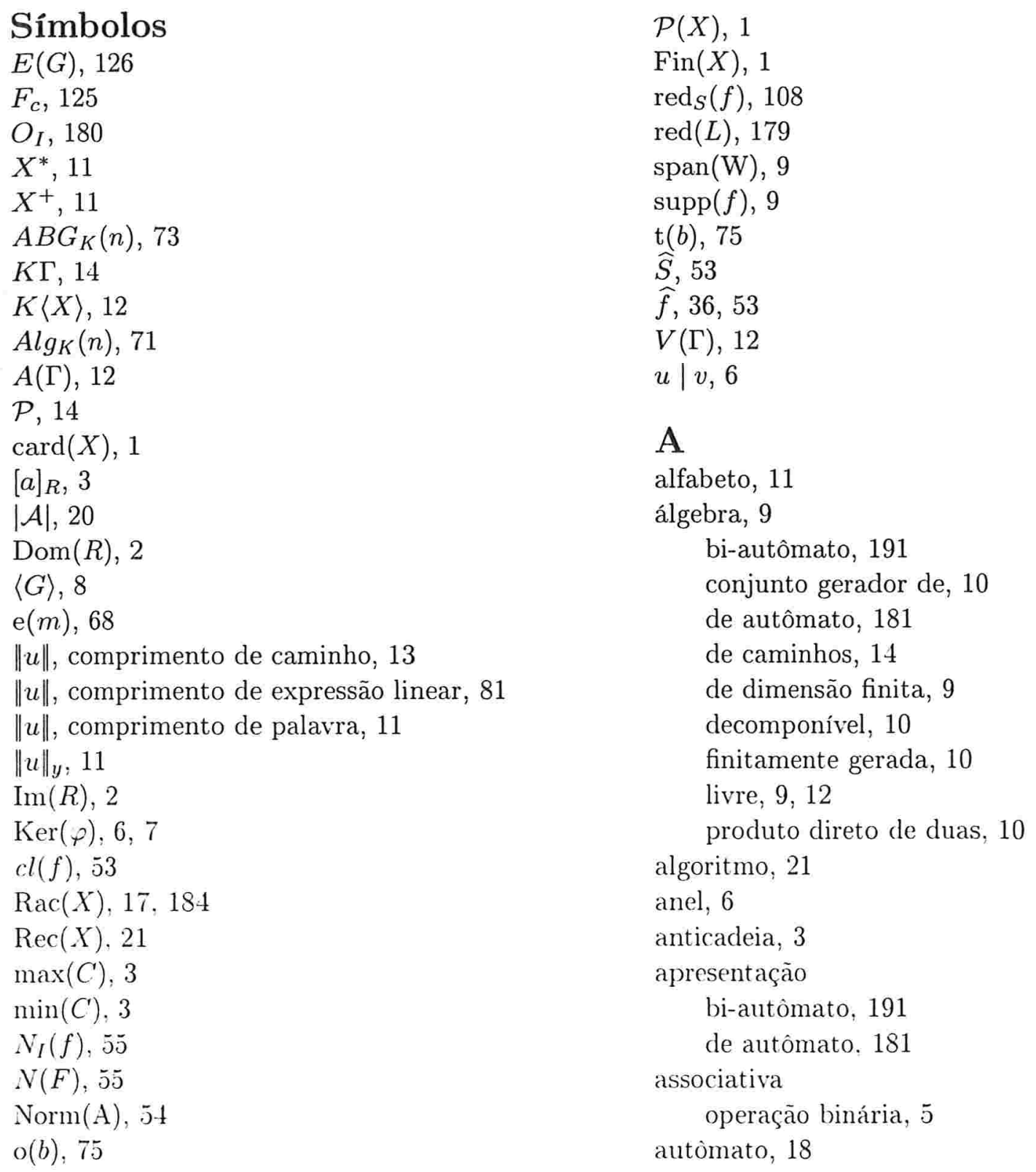


comportamento de, 20

determinístico, 20

equivalência de, 20

linguagem reconhecida por, 20

sobre monóide $M$, veja $M$-autômato

automorfismo, 5

B

Base de Gauss, 127

de espaço vetorial, 28

Base de Gröbner, 59

boa representação de, 172

comutativa, 39

infinita, exemplo de, 66

minimal, 63

reduzida, 65

e minimal, 195-196

unicidade de, 65

regular, 191

truncada, 107

universal, 68

base multiplicativa, 47

binômio puro, 78

Buchberger, 25

complexidade do Algoritmo de, 42-43

C

cadeia, 3

cadeia infinita

ascendente, 3

descendente, 3

caminho

bem-sucedido, 19

rótulo de, 19

cardinalidade, 2

classe de equivalência, 3

coeficiente líder, 53

comparável, elemento, 3

composição

de relaçōes, 2

comprimento

de caminho de grafo, veja grafo, caminho, comprimento

de expressão linear, 81

de palavra, 11

condição de cadeia descendente, 4 congruência, veja relação, congruência

conjunto

bem ordenado, 4

escalonado, 126

racional, veja racional, conjunto

reconhecivel, veja reconhecível, conjunto

tl-reduzido, veja tl-redução

uniforme, 79

coordenada líder, 28

corpo, 8

computável, 114

D

diagonal, 2

divisão

definição de, 6

domínio

de integridade, 8

de relação, 2

E

elemento

decomponível por produto, 76

idempotente, 15

inversível, 6

máximo, veja elemento, maior

mínimo, veja elemento, menor

maior, 3

maximal, 3

menor, 3

minimal, 3

neutro, veja unidade

ortogonal, 15

reduzido, 57

uniforme, 79

propriedades de, $115-118$

endomorfismo, $\bar{\jmath}$

epimorfismo, $\tilde{j}$

expressão

linear, 80

extensão

de monòmio, 68

$\mathrm{F}$

forma canônica

de caminho em $K \Gamma, 15$ 
forma normal, 55

função de transição, 18

G

grafo, 12

acíclico, 14

arco, 12

paralelo, 12

associado a base, 78

caminho, 12

comprimento de, 13

concatenação de, 13

origem e término, 12

paralelo, 13

subcaminho, 13

trivial, 13

ciclo, 14

componente de, 14

finito, 12

laço, 12

subgrafo, 14

convexo, 14

próprio, 14

vértice, 12

grau

de caminho, veja grafo, caminho, comprimento de

de palavra, veja comprimento de palavra grupo, 6

\section{$\mathrm{H}$}

homomorfismo

de álgebras, 10

de anéis, 7

kernel, 7

núcleo, veja homomorfismo de anéis, kernel

de monóides, 6

kernel, 6

núcleo, veja homomorfismo de monóides, kernel

de semigrupos, 5

\section{I}

ideal

2-nomial, 78 de álgebra, 10

conjunto gerador de, 10

finitamente gerado, 11

de anel, 7

gerado por, 8

de ordem, 54

de semigrupo, 6

conjunto gerador de, 6

finitamente gerado, 6

homogêneo, 16

monomial, 60

puramente binomial, 78

imagem

de relação, 2

incomparável

elemento, 3

indução noetheriana, 4

isomorfismo, 5

K

Kleene

estrela de, 16

teorema de, 21

L

laço

grafo, veja grafo, laço

lema

de Dickson, 37

do Diamante de Bergman, 138

linguagem, 11

reconhecível, veja reconhecível, linguagem

M

M-autòmato, 185

normalizado, 187

monóide, 6

livre, 11

propriedades básicas de(, 11

propriedades básicas de), 12

produto, 187

monômio

líder, 36

monomorfismo, 5

$\mathrm{N}$ 
noetheriano

anel, 8

$\mathrm{O}$

operação mais, 17

ordem, veja ordem parcial

admissível, 48

boa ordem, 4

computável, 114

comutativo-lexicográfica, 51

de grau e lexicografia, 34,48

de grau e lexicografia reversa, 35

de peso e lexicografia, 50

de peso e lexicografia reversa, 50

lexicográfica, 34,48

lexicográfica reversa, 35, 50

monomial, 34

seqüencial, 107

total, 3

ordem parcial, 3

origem e término

de caminho em grafo, 12

de um monômio, 75

$\mathbf{P}$

pacotes computacionais

caso comutativo, 26

caso não comutativo, 46

partes

de conjunto, 1

finitas de conjunto, 1

partição, 2

prefixo, 6

problema

computável, 23

da construção de Bases de Gröbner, 172

da igualdade, 25

da palavra em monóides, 173

da pertinència

$$
\begin{aligned}
& \text { em } K\langle X\rangle, 173 \\
& \text { em } K\left[x_{1}, \ldots, x_{n}\right], 25
\end{aligned}
$$

decidível, 23

problemas do caso não comutativo, 46-47

procedimento, 21

produto

de dois conjuntos, $\bar{j}$ projeção canônica, 8

Q

quasi-domínio, 92

quociente

anel, 8

de relação, 3

$\mathrm{R}$

racional

conjunto, 184

expressão, 17

linguagem, 17

operação, 17

relação, 188

reconhecível

conjunto, 184

linguagem, 20

redução

simples, 103

sobre um conjunto, 103

regular

linguagem, veja racional, linguagem

relação, 2

antissimétrica, 3

compatível com o produto, 3

composição, veja composição, relação

congruência, 3

gerada por, 3

de equivalência, 2

fecho multiplicativo de, 3

gerada por, 3

fecho

reflexivo, 2

reflexivo-transitivo, 3

simétrico, 2

transitivo, 3

identidade, veja diagonal

inversa, 2

racional, veja racional, relação

reflexiva, 2

simétrica, 2

transitiva, 2

relação de divisão, 131

relação de sobreposição, 130

representação 
de Gröbner, 130

forte de Gauss, 128

fraca de Gauss, 128

representante de classe, 3

$\mathrm{S}$

S-elemento, 129

existência de um número finito de, 134

S-polinômio, 40

semigrupo, 5

abeliano, veja semigrupo comutativo

comutativo, 5

conjunto gerador de, 5

finitamente gerado, 6

livre, 11

sistemas de reescrita de termos, 45-46

soma

de dois conjuntos, 5

sufixo, 6

suporte, 9

$\mathbf{T}$

teorema

da Base de Hilbert, 38

de Kleene, 21

primeiro do homomorfismo, 7

termo

líder, 53

normal, 53

tl-redução, 155

transdutor, 188

trivial

caminho

de grafo, veja grafo, caminho, trivial

U

unidade, 6 